Core crosslinked flowerlike micelles for systemic and local pharmaceutical applications

\section{Marzieh Najafi}

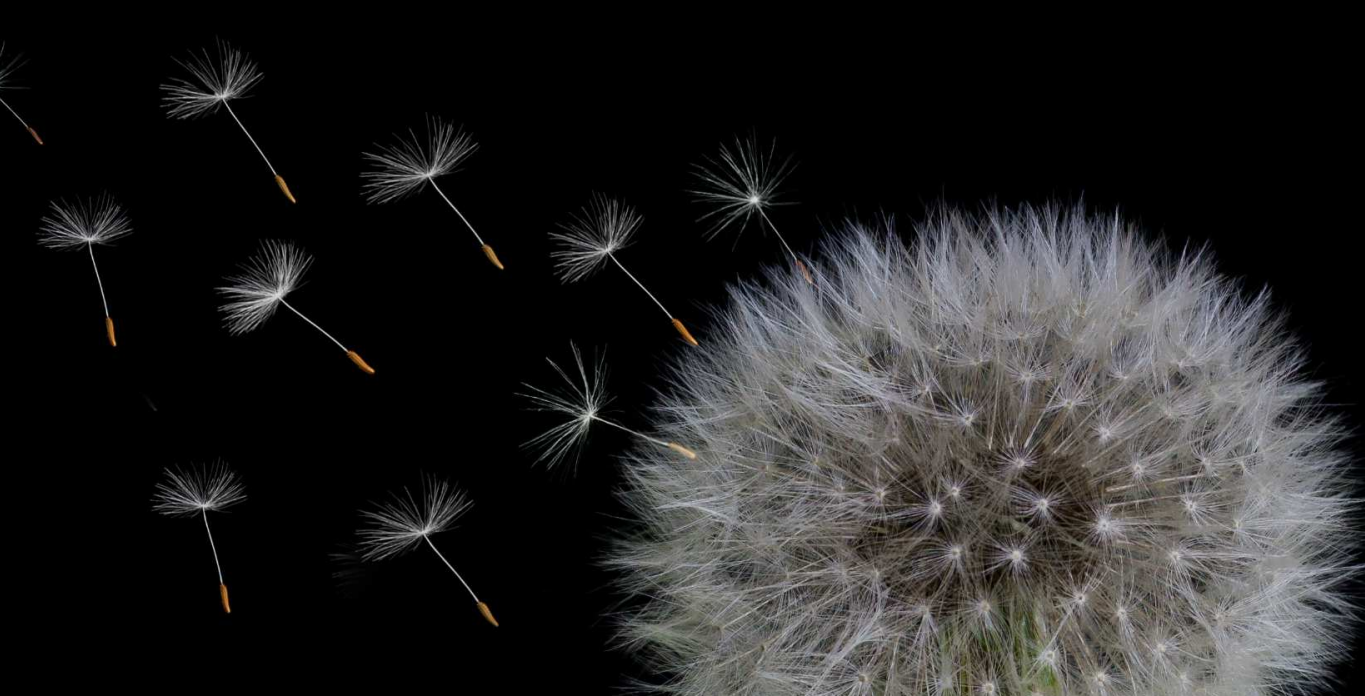

You are cordially invited to attend the public defence of the

$$
\text { thesis }
$$

\section{Core crosslinked}

flower-like

micelles for

systemic and local

pharmaceutical applications

On Monday

6th April 2020

at $2.30 \mathrm{pm}$ in

Acedemiegebouw,

Domplein 29, Utrecht

PhD candidate

* Marzieh Najafi

Marziehnajafi.sh

@gmail.com

Paranymphs

Blessing llochonwu b.c.ilochonwu@uu.nl

Lucia Martinez Jothar

Lucia.martinez.jothar @gmail.com 
Core crosslinked flower-like micelles for systemic and local pharmaceutical applications

Marzieh Najafi

2020 
The printing of this thesis was financially supported by:

Utrecht Institute for Pharmaceutical Sciences (UIPS), University of Utrecht, the Netherlands

ISBN: 978-94-6375-805-5

Cover design: Danial Keshani

Printed by: Ridderprint, www.ridderprint.nl

Najafi, M. (2020) Core crosslinked flower-like micelles for systemic and local pharmaceutical applications.

Department of Pharmaceutics, Utrecht Institute for Pharmaceutical Sciences (UIPS), Faculty of Science, University of Utrecht, the Netherlands 


\title{
Core crosslinked flower-like micelles for systemic and local pharmaceutical applications
}

\author{
Bloemvormige micellen voor systemische en lokale \\ farmaceutische toepassingen
}

(met een samenvatting in het Nederlands)

\section{Proefschrift}

ter verkrijging van de graad van doctor aan de Universiteit Utrecht op gezag van de rector magnificus, prof.dr. H.R.B.M. Kummeling, ingevolge het besluit van het college voor promoties in het openbaar te verdedigen op

maandag 6 april 2020 des middags te 2.30 uur

door

Marziyeh Najafi Shah Kouhi

geboren op 23 juli 1983

te Qom, Iran 
Promotor:

Prof. dr. ir. W. E. Hennink

\section{Copromotor:}

Dr. ir. T. Vermonden 
To my daughters, Aveed and Yara 



\section{Contents}

\section{Chapter 1}

General introduction

Chapter 2

27

Poly( $N$-isopropylacrylamide); physicochemical properties and biomedical applications

\section{Chapter 3}

Native chemical ligation for crosslinking of flower-like micelles

Chapter 4

93

The influence of PEG conformation in flower- and star-like micelles on circulation time and accelerated blood clearance

\section{Chapter 5}

Conversion of an injectable MMP-degradable hydrogel into core-crosslinked micelles

\section{Chapter 6}

Rheological properties of dense dispersions of thermosensitive core-crosslinked polymeric micelles

\section{Chapter 7}

Summary and perspectives

\section{Appendices}

Nederlandse samenvatting

Curriculum vitae

List of publications

Acknowledgments 



\section{Chapter 1}

General introduction 


\section{Nanoparticles in medicine}

Nanotechnology has resulted in many advances including nanomedicine. The National Institutes of Health of the United States and the European Science Foundation define nanomedicine as the "science that uses nanomaterials to the development of diagnosis, treatment and prevention of specific medical application"1,2. The developments in the field of nanomedicine have yielded innovations in different medical fields, including controlled drug delivery ${ }^{3,4}$, regenerative medicine ${ }^{5,6}$, molecular imaging ${ }^{7,8}{ }^{3}$, biomarkers ${ }^{9,}{ }^{10}$, and biosensing ${ }^{11,12}$. Nanoparticles $(1-100 \mathrm{~nm})$ can overcome cellular and physiological barriers and allow for imaging and diagnostics at the cellular and molecular levels. In the field of drug delivery, nanomedicine has been growing rapidly, especially for tumor therapy ${ }^{13,14}$. The researches on nanomedicines aim at addressing issues hampering the effectivity of therapeutic agents such as poor water solubility, lack of targeting capability, nonspecific distribution, systemic toxicity, and narrow therapeutic index ${ }^{15-17}$. Enormous efforts have been devoted to the development of nanocarriers with optimal sizes, shapes, and surface properties and to increase stability, prolong circulation half-life, reduce immunogenicity and improve tumor accumulation ${ }^{18-20}$ (Fig. 1). The accumulation of nanoparticles in tumor tissues usually depends on the so-called enhanced permeability and retention (EPR) effect. The unique pathophysiological characteristics of tumor tissues such as rich angiogenesis lead to high vascular density in solid tumors and the presence of large gaps between endothelial cells in tumor blood vessels contribute to the EPR effect ${ }^{21,22}$. Therefore, over time drug-loaded nanocarriers penetrate more significantly into tumor tissues than in healthy tissues. This efficient delivery of anticancer drugs administered in the form of nanoparticles has contributed to the limitation of side effects of these drugs in both preclinical and clinical settings ${ }^{16}$. Nevertheless, the EPR effect is highly variable in humans and hence the effectivity and broad applicability of nanomedicine are currently still under debate $^{17,23,24}$.

Several types of nanocarriers have been developed to improve the efficacy-safety ratio of particularly anticancer drugs including nanoparticles, dendrimers, liposomes, polymeric micelles, polymer-drug conjugates, and a number of them have reached the clinical development stage and some made it successfully to the market ${ }^{23,25}$. 


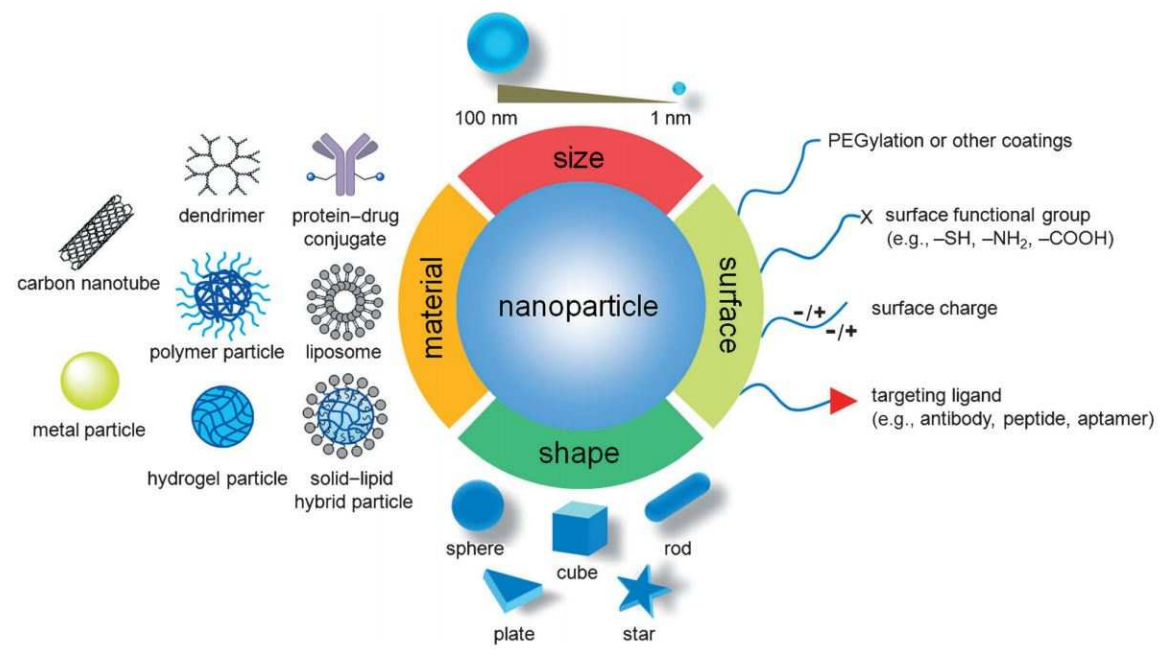

Figure 1. An overview of different types of nanoparticles that have been explored as carriers for drug delivery in cancer therapy. Reproduced with permission from ref ${ }^{15}$.

\section{Polymeric micelles}

An important class of nanoparticles that have been extensively studied for drug delivery applications are polymeric micelles $(P M)^{26-28}$. They are formed by the self-assembly of amphiphilic block (co)polymers in aqueous solutions at a concentration above the critical micelle concentration (CMC) resulting in nanosized particles with a hydrophilic shell and hydrophobic core ${ }^{28}$. A great variety of amphiphilic block copolymers of different architectures have been used to prepare micelles. The two most common designs are $A B$ and $A B A$ amphiphilic block (co)polymers in which the A block(s) are hydrophobic and the B block is hydrophilic resulting in so-called star-like and flower-like micelles, respectively ${ }^{29}$. In general, flower-like micelles display lower CMC values and higher stability than star-like micelles ${ }^{30,31}$.

In a typical core-shell micellar drug delivery system, the hydrophilic shell stabilizes the micellar structure and the hydrophobic core can solubilize hydrophobic drugs. The most frequently used polymer as the hydrophilic outer shell is polyethylene glycol (PEG) ${ }^{28,32}$. This polymer is highly hydrated and displays excellent protein repellent properties thereby minimizing recognition of micelles by the mononuclear phagocyte system (MPS) ${ }^{33}$ which are often referred to as stealth-like properties ${ }^{34-36}$. Long circulation of micelles is a prerequisite for EPR-mediated passive drug targeting to tumors and to other inflammation sites ${ }^{37}$. Both the core and the shell of micelles can be modified in different ways e.g. by crosslinking $38-40$ and decorating the shell with targeting ligands ${ }^{41,42}$ to efficiently improve circulation half-life. 


\section{Core crosslinking of micelles}

Core crosslinking $(\mathrm{CCL})$ of micelles is done after micellization and prevents micelle dissociation upon dilution below the $\mathrm{CMC}^{39}$. For covalent crosslinking of the micellar core, the reactive groups should be present in the side chains or end-group of the hydrophobic block. Notably, the chemical properties of these functional groups should not hamper micelle formation. Diverse methods of core-crosslinking have been exploited including radial polymerization ${ }^{38,43,44}$, crosslinking by bi- or multi-functional crosslinkers e.g. diamine or cystamine ${ }^{45,46}$, disulfide bond formation ${ }^{47}$, thiol-ene reaction ${ }^{48}$, and alkyne-azide cycloaddition reaction (CuAAC) ${ }^{49}$. Some of these technologies have shown to indeed increase the stability of micelles in biological media which can lead to improved circulation kinetics 4 , 50,51. Despite all the advances in this field, there are some limitations in the mentioned methods for biomedical applications, such as exposure to radicals, oxidative conditions, use of organic solvents, and the presence of (toxic) catalysts. Besides, the consumption of functional groups in the micellar core or shell for crosslinking limits further modification steps e.g. drug or dye conjugations. Hence, exploiting a crosslinking method that retains functional groups in the micellar structure and is applicable in physiological conditions is of high interest.

\section{Native chemical ligation}

Native chemical ligation (NCL) has been developed as highly efficient and robust linking method of unprotected peptides and protein segments to generate larger constrtucts ${ }^{52}$. Figure 2 shows the NCL mechanism in which a chemoselective reaction of an $N$-terminal cysteine with thioester moiety gives an intermediate thioester-linked species. Spontaneous rearrangement of this transient group by an intramolecular $\mathrm{S}, \mathrm{N}$-acyl shift yields a product with a native peptide bond at the ligation site. Native chemical ligation is highly specific and hence only a single product is formed even in the presence of additional cysteine residues present in e.g. proteins. NCL has been used mostly in the field of protein and peptide synthesis and modification, and more recently this method gained interest for coupling of synthetic polymers e.g. for the formation of in situ crosslinked hydrogels ${ }^{53-55}$. This crosslinking method allows the application of injectable hydrogels suitable for minimally invasive treatments. The free thiol resulting from this reaction can be used for further conjugation of desirable molecules via e.g. disulfide bond, Michael addition, and thiol-ene reactions. 


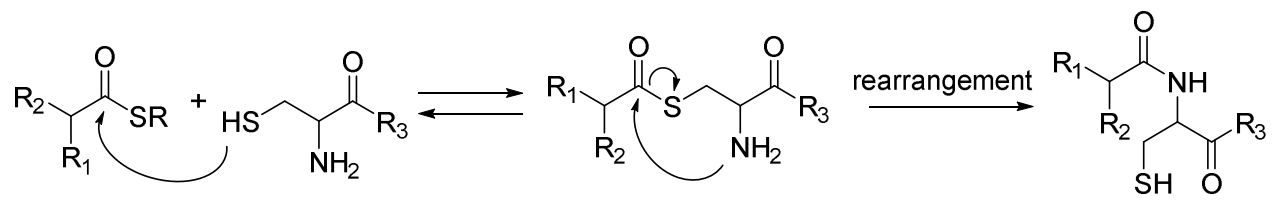

Compound 1

Compound 2

Figure 2: The mechanism of native chemical ligation. Compound 1, which contains a thioester at the $\alpha$-carboxyl group, undergoes nucleophilic attack by the side chain of the cysteine residue at the amino terminal of compound 2 . The intermediate thioester ligation product undergoes a rapid intramolecular reaction to yield a product with a native peptide bond at the ligation site.

\section{Accelerated blood clearance}

As discussed, the stealth-like properties of PEG are ascribed to its hydrophilic and neutral nature, which diminishes the binding of the plasma proteins and increases the circulation time of PEGylated particles ${ }^{56}$. However, there are recent reports demonstrating that PEGylation does not completely avoid the binding of plasma proteins, but instead the relatively long circulation time of pegylated nanoparticles is caused by the preferential adsorption of specific proteins such as clusterin ${ }^{57}$ that prevent binding of opsonins and inhibit recognition of these particles by $\mathrm{MPS}^{58,59}$.

Generally speaking PEGylated particles are less immunogenic than their non-PEGylated counterparts, however, several reports have shown that intravenous injection (i.v) of a PEGylated nanomedicine triggers the fast

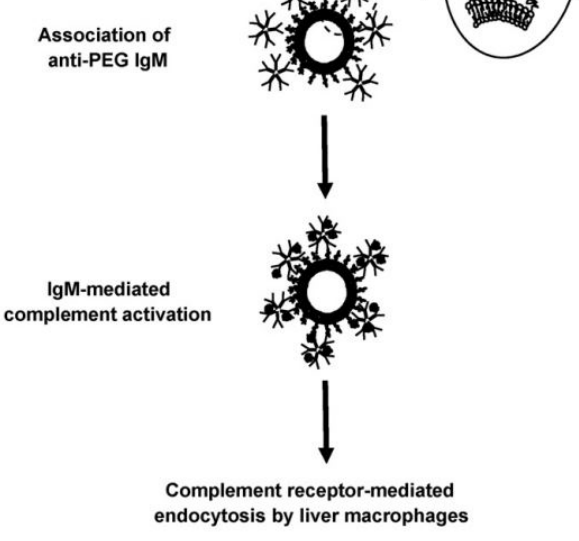

Figure 3: Representation of the sequence of events leading from anti-PEG IgM induction to accelerated clearance of PEGylated liposomes. Reproduced by permission from ref $^{63}$. clearance of subsequently injected PEGylated nanomedicine doses ${ }^{60}$. This effect is known as the accelerated blood clearance $(A B C)$ phenomenon and has been observed for several types of PEGylated nanomedicines such as liposomes, protein-conjugates, and micelles ${ }^{61-63}$. Injection of the first dose of PEGylated particles triggers the production of anti-PEG IgM by 
splenic marginal zone B cells ${ }^{61,64}$. When plasma anti-PEG IgM titers are high (5-21 days after the first injection), injection of a subsequent dose of PEGylated particles results in binding of anti-PEG IgM selectively to the particle surface leading to substantial complement activation and fast clearance of this dose (Fig. 3$)^{63,65,66}$. The magnitude of the $A B C$ phenomenon depends on the physicochemical properties of injected (first dose) PEGylated particles such as size ${ }^{67}$, PEG density and length ${ }^{68}$, PEG end group ${ }^{69}, 70$, etc., as well as the time interval between the injections ${ }^{63}$. The $A B C$ phenomenon occurs in animals e.g. mice ${ }^{71}$, rats $^{60}$, beagle dogs ${ }^{72}$, cynomolgus monkeys, and mini pigs ${ }^{73}$. In a number of publications, it has been shown that the induction of anti-PEG antibodies occurs in humans treated with PEGylated therapeutics or those persistently exposed to PEG-containing products ${ }^{74-76}$. Therefore, finding an optimal nanoparticle design that can address the observed immunogenicity of PEGylated particles has received much attention in recent years.

\section{Hydrogels as smart materials}

Hydrogels are three-dimensional networks of hydrophilic polymers hold together by physical or chemical crosslinks ${ }^{77,78}$. These materials are capable of absorbing large amounts of water while maintaining their structure. These materials exhibit several interesting properties for applications in controlled drug delivery systems ${ }^{79,80}$, as cell culture matrices ${ }^{81-}$ ${ }^{83}$, and in tissue engineering constructs ${ }^{84,85}$. Both synthetic and natural polymers have been exploited for hydrogel formation each having advantages and disadvantages regarding e.g. cytocompatibility, tunability of mechanical properties, and biodegradability ${ }^{86-88}$. Hydrogels can be composed of polymers that change their properties in response to a single or multiple stimuli. These so-called 'smart' or stimuli-responsive polymers represent a growing cadre of materials exploited for various biomedical application $s^{89,90}$. Several types of stimuliresponsive hydrogels such as termperature ${ }^{91}$, light ${ }^{92}, 93$, enzyme ${ }^{94}, \mathrm{pH}^{95}, 96$ have been developed for drug delivery applications. The functionality of these materials is reflected in their programmability. For instance, enzyme responsive hydrogels protect their payload from premature release as long as the corresponding enzyme is absent in the surrounding environment. Elevated concentrations of e.g. proteolytic enzymes such as MMPs (matrix metalloproteinases) present in tumors or inflamed tissues, can trigger hydrogel degradation, and release of loaded therapeutics ${ }^{97}$. 


\section{Colloidal suspensions}

Colloidal suspensions are composed of small (10 $\mathrm{nm}$ to $10 \mu \mathrm{m}$ radius) solid particles dispersed in a liquid phase with a variety of interparticle interactions including repulsive (e.g. electrostatic forces) and attractive (e.g. van der Waals) $)^{98}$ forces. Colloidal suspensions can be categorized into two main groups, hard (e.g. carbon black $)^{99}$ and soft colloidal particles (e.g. microgel) $)^{100}$. In this section, the focus is on soft deformable colloidal hydrogel particles ${ }^{101}$.

Poly(N-isopropylacrylamide) (PNIPAM) is a thermo-responsive polymer having a lower critical solution temperature (LCST) around

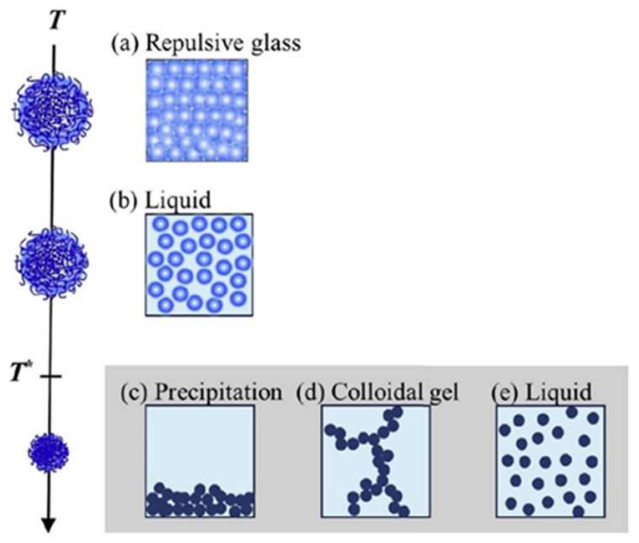

Figure 4: A variety of states of repulsive glass composed of thermosensitive microgels subjected to heating. Reproduced by permission from ref ${ }^{104}$. $32{ }^{\circ} \mathrm{C}$ in aqueous solution. Below this temperature, the polymeric chains are strongly hydrated and therefore soluble in water, while upon increasing the temperature above the LCST, dehydration occurs and thus the polymers are insoluble (see also chapter 2 of this thesis) ${ }^{102}$. In a microgel crosslinked network of PNIPAM and related copolymers, chains undergo a volume-phase transition between a swollen and collapsed state at a temperature close to the LCST, also defined as the volume phase transition temperature (VPTT $)^{103}$. In a concentrated suspension of the PNIPAM based microgels, at T < VPTT, the steric hindrance of the swollen polymeric chains results in repulsive interactions between the particles. On the other hand collapse of polymeric chains at T > VPTT results in hydrophobic and van der Waals interactions, and consequently attractive interparticle interactions ${ }^{104}$. Concentrated dispersions of PNIPAM based microgels show a remarkable change in their rheological properties when subjected to increasing temperature owing to a switch in the interparticle interactions from repulsive to attractive. A so-called repulsive glass that behaves as a solid with yield stress is formed at $\mathrm{T}<$ VPTT when repulsive interactions are high. At T > VPTT, a variety of states have been reported depending on the magnitude of repulsive and attractive interactions. A balance between attractive and repulsive interactions can result in the formation of a so-called colloidal gel ${ }^{105}$, while excessive repulsive interactions lead to a stable dispersion of the microgel (liquid) ${ }^{106}$, whereas excessive attractive interactions result in macroscopic precipitation (Fig. 4) ${ }^{104}$. Therefore, comprehensive understanding of the role of polymer 
structures in interparticle interactions, physical properties, and rheological characterization of colloidal suspensions provides insights for developing new functional materials for pharmaceutical and biomedical applications.

\section{Aim of this thesis}

In this thesis, the pharmaceutical suitability of core crosslinked flower-like micelles for systemic and local applications was investigated. In this regard, circulation kinetics and occurrence of the $A B C$ phenomenon upon repeated injections of core crosslinked flowerand star-like micelles were investigated. Moreover, the potential of core-crosslinked flowerlike micelles for the formulation of stimuli-responsive (either enzyme or temperature) hydrogels was studied.

\section{Thesis outline}

Chapter 2 gives a literature overview of physicochemical properties of PNIPAM based materials and their biomedical and pharmaceutical applications. Combining thermosensitive properties of PNIPAM with other stimuli-responsive materials are discussed and examples of PNIPAM-protein/peptide hybrid materials are given.

Chapter 3 describes the formation of flower-like micelles using two complementary ABA triblock copolymers having a B block of PEG and A blocks of either P(NIPAM-co-N-(2hydroxypropyl) methacrylamide-cysteine (HPMA-Cys)) or P(NIPAM-co- N-(2hydroxypropyl)methacrylamide-ethylthio glycolate succinic acid (HPMA-ETSA). This chapter describes native chemical ligation between cysteine and thioester functionalities in the micellar core for effective core crosslinking of flower-like micelles.

Chapter 4 explores the effect of PEG conformation in the corona of core-crosslinked micelles on circulation kinetics, biodistribution, and the occurrence of the $A B C$ phenomenon. In this study, star-like micelles were prepared using two complementary AB diblock copolymers of PEG-P(NIPAM-Co-HPMA-ETSA) and PEG-P(NIPAM-Co-HPMA-Cys). The physical characteristics of flower-like micelles (discussed in chapter 3) having a looped PEG conformation were compared to star-like micelles displaying linear PEG chains in the corona. Circulation kinetics of fluorescently labeled micelles were assessed in immunocompetent $B A L B / c$ mice. To investigate the occurrence of the $A B C$ phenomenon, mice were subjected to a second injection and the blood samples were analyzed for anti-PEG IgM titers by means of ELISA. 
Chapter 5 introduces an injectable enzyme degradable hydrogel composed of flower-like micelles held together by a MMP cleavable crosslinker. The effect of enzyme concentration on the degradation profile of the hydrogel and its conversion into CCL micelles was investigated. To explore the possible application of the hydrogel for intracellular drug delivery, the cellular uptake of the released CCL micelles was studied using HeLa cells.

Chapter 6 reports the size and configurational changes of the CCL flower-like micelles upon varying temperatures using a rigidochromic fluorescent molecule conjugated in the micellar core. Furthermore, the rheological properties of dense dispersions of CCL flower-like micelles as a function of temperature are investigated. Also, a physical model was used to describe the storage modulus $\left(G^{\prime}\right)$ of micellar dispersions at different concentrations and compared to the experimental data.

Chapter 7 summarizes the major findings of this thesis. Finally, some suggestions for the future development of the formulations are given and potential applications are outlined. 


\section{References}

1. Oberdörster, G., Safety assessment for nanotechnology and nanomedicine: concepts of nanotoxicology. Journal of Internal Medicine 2010, 267 (1), 89-105.

2. Foundation, E. E. S. Nanomedicine, an ESF-European Medical Research Councils (EMRC) Forward Look Report, ESF; France, 2004.

3. Parveen, S.; Misra, R.; Sahoo, S. K., Nanoparticles: a boon to drug delivery, therapeutics, diagnostics and imaging. Nanomedicine: Nanotechnology, Biology and Medicine 2012, 8 (2), 147-166.

4. Tran, S.; DeGiovanni, P.-J.; Piel, B.; Rai, P., Cancer nanomedicine: a review of recent success in drug delivery. Clinical and Translational Medicine 2017, 6 (1), 44.

5. Zor, F.; Selek, F. N.; Orlando, G.; Williams, D. F., Biocompatibility in regenerative nanomedicine. Nanomedicine 2019, 14 (20), 2763-2775.

6. Yang, Y.; Chawla, A.; Zhang, J.; Esa, A.; Jang, H. L.; Khademhosseini, A., Chapter 29 - Applications of Nanotechnology for Regenerative Medicine; Healing Tissues at the Nanoscale. In Principles of Regenerative Medicine (Third Edition), Atala, A.; Lanza, R.; Mikos, A. G.; Nerem, R., Eds. Academic Press: Boston, 2019; pp 485-504.

7. Xia, Y.; Xu, C.; Zhang, X.; Ning, P.; Wang, Z.; Tian, J.; Chen, X., Liposome-based probes for molecular imaging: from basic research to the bedside. Nanoscale 2019, 11 (13), 5822-5838.

8. Bouché, M.; Hsu, J. C.; Dong, Y. C.; Kim, J.; Taing, K.; Cormode, D. P., Recent Advances in Molecular Imaging with Gold Nanoparticles. Bioconjugate Chemistry 2019, DOI: 10.1021/acs.bioconjchem.9b00669.

9. Ye, F.; Zhao, Y.; El-Sayed, R.; Muhammed, M.; Hassan, M., Advances in nanotechnology for cancer biomarkers. Nano Today 2018, 18, 103-123.

10. Hadjidemetriou, M.; Al-ahmady, Z.; Buggio, M.; Swift, J.; Kostarelos, K., A novel scavenging tool for cancer biomarker discovery based on the blood-circulating nanoparticle protein corona. Biomaterials 2019, 188, 118-129.

11. Zhang, X.; Guo, Q.; Cui, D., Recent Advances in Nanotechnology Applied to Biosensors. Sensors 2009, 9 (2), 1033-1053.

12. Holzinger, M.; Le Goff, A.; Cosnier, S., Nanomaterials for biosensing applications: a review. Frontiers in Chemistry 2014, 2, article 63 (page 1-10).

13. Bor, G.; Mat Azmi, I. D.; Yaghmur, A., Nanomedicines for cancer therapy: current status, challenges and future prospects. Therapeutic Delivery 2019, 10 (2), 113-132.

14. van der Meel, R.; Sulheim, E.; Shi, Y.; Kiessling, F.; Mulder, W. J. M.; Lammers, T., Smart cancer nanomedicine. Nature Nanotechnology 2019, 14 (11), 1007-1017.

15. Sun, T.; Zhang, Y. S.; Pang, B.; Hyun, D. C.; Yang, M.; Xia, Y., Engineered 
Nanoparticles for Drug Delivery in Cancer Therapy. Angewandte Chemie International Edition 2014, 53 (46), 12320-12364.

16. Couvreur, P.; Vauthier, C., Nanotechnology: Intelligent Design to Treat Complex Disease. Pharmaceutical Research 2006, 23 (7), 1417-1450.

17. Couvreur, P., Nanomedicine: From where are we coming and where are we going? Journal of Controlled Release 2019, 311-312, 319-321.

18. Steichen, S. D.; Caldorera-Moore, M.; Peppas, N. A., A review of current nanoparticle and targeting moieties for the delivery of cancer therapeutics. European Journal of Pharmaceutical Sciences 2013, 48 (3), 416-427.

19. Whitesides, G. M., The 'right' size in nanobiotechnology. Nature Biotechnology 2003, 21 (10), 1161-1165.

20. Ferrari, M., Cancer nanotechnology: opportunities and challenges. Nature Reviews Cancer 2005, 5 (3), 161-171.

21. Maeda, H.; Wu, J.; Sawa, T.; Matsumura, Y.; Hori, K., Tumor vascular permeability and the EPR effect in macromolecular therapeutics: a review. Journal of Controlled Release 2000, 65 (1), 271-284.

22. Fang, J.; Nakamura, H.; Maeda, H., The EPR effect: Unique features of tumor blood vessels for drug delivery, factors involved, and limitations and augmentation of the effect. Advanced Drug Delivery Reviews 2011, 63 (3), 136-151.

23. Danhier, F., To exploit the tumor microenvironment: Since the EPR effect fails in the clinic, what is the future of nanomedicine? Journal of Controlled Release 2016, 244, 108-121. 24. Park, K., The beginning of the end of the nanomedicine hype. Journal of Controlled Release 2019, 305, 221-222.

25. Wicki, A.; Witzigmann, D.; Balasubramanian, V.; Huwyler, J., Nanomedicine in cancer therapy: Challenges, opportunities, and clinical applications. Journal of Controlled Release 2015, 200, 138-157.

26. Kataoka, K.; Harada, A.; Nagasaki, Y., Block copolymer micelles for drug delivery: Design, characterization and biological significance. Advanced Drug Delivery Reviews 2012, 64, 37-48.

27. Talelli, M.; Hennink, W. E., Thermosensitive polymeric micelles for targeted drug delivery. Nanomedicine 2011, 6 (7), 1245-1255.

28. Cabral, H.; Miyata, K.; Osada, K.; Kataoka, K., Block Copolymer Micelles in Nanomedicine Applications. Chemical Reviews 2018, 118 (14), 6844-6892.

29. de Graaf, A. J.; Boere, K. W.; Kemmink, J.; Fokkink, R. G.; van Nostrum, C. F.; Rijkers, D. T.; van der Gucht, J.; Wienk, H.; Baldus, M.; Mastrobattista, E.; Vermonden, T.; Hennink, W. E., Looped structure of flowerlike micelles revealed by $1 \mathrm{H}$ NMR relaxometry and light scattering. Langmuir 2011, 27 (16), 9843-9848. 
30. Lee, E. S.; Oh, K. T.; Kim, D.; Youn, Y. S.; Bae, Y. H., Tumor pH-responsive flowerlike micelles of poly(L-lactic acid)-b-poly(ethylene glycol)-b-poly(L-histidine). Journal of Controlled Release 2007, 123 (1), 19-26.

31. Oh, K. T.; Oh, Y. T.; Oh, N.-M.; Kim, K.; Lee, D. H.; Lee, E. S., A smart flower-like polymeric micelle for $\mathrm{pH}$-triggered anticancer drug release. International Journal of Pharmaceutics 2009, 375 (1), 163-169.

32. Gaucher, G.; Dufresne, M.-H.; Sant, V. P.; Kang, N.; Maysinger, D.; Leroux, J.-C., Block copolymer micelles: preparation, characterization and application in drug delivery. Journal of Controlled Release 2005, 109 (1), 169-188.

33. Storm, G.; Belliot, S. O.; Daemen, T.; Lasic, D. D., Surface modification of nanoparticles to oppose uptake by the mononuclear phagocyte system. Advanced Drug Delivery Reviews 1995, 17 (1), 31-48.

34. Bazile, D.; Prud'homme, C.; Bassoullet, M. T.; Marlard, M.; Spenlehauer, G.; Veillard, M., Stealth Me. PEG-PLA Nanoparticles Avoid Uptake by the Mononuclear Phagocytes System. Journal of Pharmaceutical Sciences 1995, 84 (4), 493-498.

35. Jin, Q.; Deng, Y.; Chen, X.; Ji, J., Rational Design of Cancer Nanomedicine for Simultaneous Stealth Surface and Enhanced Cellular Uptake. ACS Nano 2019, 13 (2), 954977.

36. Sethuraman, V. A.; Bae, Y. H., TAT peptide-based micelle system for potential active targeting of anti-cancer agents to acidic solid tumors. Journal of Controlled Release 2007, $118(2), 216-224$.

37. Eetezadi, S.; Ekdawi, S. N.; Allen, C., The challenges facing block copolymer micelles for cancer therapy: In vivo barriers and clinical translation. Advanced Drug Delivery Reviews 2015, 91, 7-22.

38. Talelli, M.; Iman, M.; Varkouhi, A. K.; Rijcken, C. J. F.; Schiffelers, R. M.; Etrych, T.; Ulbrich, K.; van Nostrum, C. F.; Lammers, T.; Storm, G.; Hennink, W. E., Corecrosslinked polymeric micelles with controlled release of covalently entrapped doxorubicin. Biomaterials 2010, 31 (30), 7797-7804.

39. Talelli, M.; Barz, M.; Rijcken, C. J. F.; Kiessling, F.; Hennink, W. E.; Lammers, T., Core-crosslinked polymeric micelles: Principles, preparation, biomedical applications and clinical translation. Nano Today 2015, 10 (1), 93-117.

40. Wang, L.; Zhang, J.; Song, M.; Tian, B.; Li, K.; Liang, Y.; Han, J.; Wu, Z., A shell-crosslinked polymeric micelle system for $\mathrm{pH} /$ redox dual stimuli-triggered DOX ondemand release and enhanced antitumor activity. Colloids and Surfaces B: Biointerfaces 2017, 152, 1-11.

41. Kedar, U.; Phutane, P.; Shidhaye, S.; Kadam, V., Advances in polymeric micelles for drug delivery and tumor targeting. Nanomedicine 2010, 6 (6), 714-729. 
42. Jiang, X.; Liu, S.; Narain, R., Degradable thermoresponsive core cross-linked micelles: fabrication, surface functionalization, and biorecognition. Langmuir 2009, 25 (23), 1334413350.

43. Blencowe, A.; Tan, J. F.; Goh, T. K.; Qiao, G. G., Core cross-linked star polymers via controlled radical polymerisation. Polymer 2009, 50 (1), 5-32.

44. Rijcken, C. J.; Snel, C. J.; Schiffelers, R. M.; van Nostrum, C. F.; Hennink, W. E., Hydrolysable core-crosslinked thermosensitive polymeric micelles: Synthesis, characterisation and in vivo studies. Biomaterials 2007, 28 (36), 5581-5593.

45. Bronich, T. K.; Keifer, P. A.; Shlyakhtenko, L. S.; Kabanov, A. V., Polymer Micelle with Cross-Linked Ionic Core. Journal of the American Chemical Society 2005, 127 (23), 8236-8237. 46. Du, A. W.; Lu, H.; Stenzel, M., Stabilization of Paclitaxel-Conjugated Micelles by CrossLinking with Cystamine Compromises the Antitumor Effects against Two- and ThreeDimensional Tumor Cellular Models. Molecular Pharmaceutics 2016, 13 (11), 3648-3656.

47. Wu, L.; Zou, Y.; Deng, C.; Cheng, R.; Meng, F.; Zhong, Z., Intracellular release of doxorubicin from core-crosslinked polypeptide micelles triggered by both $\mathrm{pH}$ and reduction conditions. Biomaterials 2013, 34 (21), 5262-5272.

48. Huang, Y.; Sun, R.; Luo, Q.; Wang, Y.; Zhang, K.; Deng, X.; Zhu, W.; Li, X.; Shen, Z., In situ fabrication of paclitaxel-loaded core-crosslinked micelles via thiol-ene "click" chemistry for reduction-responsive drug release. Journal of Polymer Science Part A: Polymer Chemistry 2016, 54 (1), 99-107.

49. Zhang, J.; Zhou, Y.; Zhu, Z.; Ge, Z.; Liu, S., Polyion Complex Micelles Possessing Thermoresponsive Coronas and Their Covalent Core Stabilization via "Click" Chemistry. Macromolecules 2008, 41 (4), 1444-1454.

50. Gu, Z.; Wang, X.; Cheng, R.; Cheng, L.; Zhong, Z., Hyaluronic acid shell and disulfide-crosslinked core micelles for in vivo targeted delivery of bortezomib for the treatment of multiple myeloma. Acta Biomaterialia 2018, 80, 288-295.

51. Fan, W.; Zhang, L.; Li, Y.; Wu, H., Recent Progress of Crosslinking Strategies for Polymeric Micelles with Enhanced Drug Delivery in Cancer Therapy. Current Medicinal Chemistry 2019, 26 (13), 2356-2376.

52. Dawson, P. E.; Muir, T. W.; Clark-Lewis, I.; Kent, S. B., Synthesis of proteins by native chemical ligation. Science 1994, 266 (5186), 776-779.

53. Boere, K. W. M.; Soliman, B. G.; Rijkers, D. T. S.; Hennink, W. E.; Vermonden, T., Thermoresponsive Injectable Hydrogels Cross-Linked by Native Chemical Ligation. Macromolecules 2014, 47 (7), 2430-2438.

54. Hu, B.-H.; Su, J.; Messersmith, P. B., Hydrogels Cross-Linked by Native Chemical Ligation. Biomacromolecules 2009, 10 (8), 2194-2200.

55. Boere, K. W. M.; van den Dikkenberg, J.; Gao, Y.; Visser, J.; Hennink, W. E.; 
Vermonden, T., Thermogelling and Chemoselectively Cross-Linked Hydrogels with Controlled Mechanical Properties and Degradation Behavior. Biomacromolecules 2015, 16 (9), 2840-2851.

56. Hamidi, M.; Azadi, A.; Rafiei, P., Pharmacokinetic Consequences of Pegylation. Drug Delivery 2006, 13 (6), 399-409.

57. Schöttler, S.; Becker, G.; Winzen, S.; Steinbach, T.; Mohr, K.; Landfester, K.; Mailänder, V.; Wurm, F. R., Protein adsorption is required for stealth effect of poly(ethylene glycol)- and poly(phosphoester)-coated nanocarriers. Nature Nanotechnology 2016, 11 (4), 372-377.

58. Verhoef, J. J. F.; Anchordoquy, T. J., Questioning the use of PEGylation for drug delivery. Drug Delivery and Translational Research 2013, 3 (6), 499-503.

59. Butcher, N. J.; Mortimer, G. M.; Minchin, R. F., Unravelling the stealth effect. Nature Nanotechnology 2016, 11, 310-311.

60. Dams, E. T. M.; Laverman, P.; Oyen, W. J. G.; Storm, G.; Scherphof, G. L.; van der Meer, J. W. M.; Corstens, F. H. M.; Boerman, O. C., Accelerated Blood Clearance and Altered Biodistribution of Repeated Injections of Sterically Stabilized Liposomes. Journal of Pharmacology and Experimental Therapeutics 2000, 292 (3), 1071-1079.

61. Abu Lila, A. S.; Kiwada, H.; Ishida, T., The accelerated blood clearance (ABC) phenomenon: Clinical challenge and approaches to manage. Journal of Controlled Release 2013, 172 (1), 38-47.

62. Richter, A. W.; Åkerblom, E., Antibodies against Polyethylene Glycol Produced in Animals by Immunization with Monomethoxy Polyethylene Glycol Modified Proteins. International Archives of Allergy and Immunology 1983, 70 (2), 124-131.

63. Ishida, T.; Kiwada, H., Accelerated blood clearance (ABC) phenomenon upon repeated injection of PEGylated liposomes. International Journal of Pharmaceutics 2008, 354 (1), 5662.

64. Koide, H.; Asai, T.; Hatanaka, K.; Akai, S.; Ishii, T.; Kenjo, E.; Ishida, T.; Kiwada, H.; Tsukada, H.; Oku, N., T cell-independent B cell response is responsible for $A B C$ phenomenon induced by repeated injection of PEGylated liposomes. International Journal of Pharmaceutics 2010, 392 (1), 218-223.

65. Ishida, T.; Kiwada, H., Anti-polyethyleneglycol Antibody Response to PEGylated Substances. Biological and Pharmaceutical Bulletin 2013, 36 (6), 889-891.

66. Shiraishi, K.; Yokoyama, M., Toxicity and immunogenicity concerns related to PEGylated-micelle carrier systems: a review. Science and Technology of Advanced Materials 2019, 20 (1), 324-336.

67. Koide, H.; Asai, T.; Hatanaka, K.; Urakami, T.; Ishii, T.; Kenjo, E.; Nishihara, M.; Yokoyama, M.; Ishida, T.; Kiwada, H.; Oku, N., Particle size-dependent triggering of 
accelerated blood clearance phenomenon. International Journal of Pharmaceutics 2008, 362 (1), 197-200.

68. Ishida, T.; Harada, M.; Wang, X. Y.; Ichihara, M.; Irimura, K.; Kiwada, H., Accelerated blood clearance of PEGylated liposomes following preceding liposome injection: Effects of lipid dose and PEG surface-density and chain length of the first-dose liposomes. Journal of Controlled Release 2005, 105 (3), 305-317.

69. Sherman, M. R.; Williams, L. D.; Sobczyk, M. A.; Michaels, S. J.; Saifer, M. G. P., Role of the Methoxy Group in Immune Responses to mPEG-Protein Conjugates. Bioconjugate Chemistry 2012, 23 (3), 485-499.

70. Shimizu, T.; Abu Lila, A. S.; Fujita, R.; Awata, M.; Kawanishi, M.; Hashimoto, Y.; Okuhira, K.; Ishima, Y.; Ishida, T., A hydroxyl PEG version of PEGylated liposomes and its impact on anti-PEG IgM induction and on the accelerated clearance of PEGylated liposomes. European Journal of Pharmaceutics and Biopharmaceutics 2018, 127, 142-149.

71. Ishida, T.; Ichikawa, T.; Ichihara, M.; Sadzuka, Y.; Kiwada, H., Effect of the physicochemical properties of initially injected liposomes on the clearance of subsequently injected PEGylated liposomes in mice. Journal of Controlled Release 2004, 95 (3), 403-412.

72. Suzuki, T.; Ichihara, M.; Hyodo, K.; Yamamoto, E.; Ishida, T.; Kiwada, H.; Ishihara, H.; Kikuchi, H., Accelerated blood clearance of PEGylated liposomes containing doxorubicin upon repeated administration to dogs. International Journal of Pharmaceutics 2012, 436 (1), 636-643.

73. Suzuki, T.; Ichihara, M.; Hyodo, K.; Yamamoto, E.; Ishida, T.; Kiwada, H.; Kikuchi, H.; Ishihara, H., Influence of dose and animal species on accelerated blood clearance of PEGylated liposomal doxorubicin. International Journal of Pharmaceutics 2014, 476 (1), 205-212.

74. Garay, R. P.; El-Gewely, R.; Armstrong, J. K.; Garratty, G.; Richette, P., Antibodies against polyethylene glycol in healthy subjects and in patients treated with PEG-conjugated agents. Expert Opinion on Drug Delivery 2012, 9 (11), 1319-1323.

75. Armstrong, J. K., The occurrence, induction, specificity and potential effect of antibodies against poly(ethylene glycol). In PEGylated Protein Drugs: Basic Science and Clinical Applications, Veronese, F. M., Ed. Birkhäuser Basel: Basel, 2009; pp 147-168.

76. Le, Y.; Toyofuku, W. M.; Scott, M. D., Immunogenicity of murine mPEG-red blood cells and the risk of anti-PEG antibodies in human blood donors. Experimental Hematology 2017, 47, 36-47.

77. Hoffman, A. S., Hydrogels for biomedical applications. Advanced Drug Delivery Reviews 2012, 64, 18-23.

78. Buwalda, S. J.; Boere, K. W. M.; Dijkstra, P. J.; Feijen, J.; Vermonden, T.; Hennink, W. E., Hydrogels in a historical perspective: From simple networks to smart materials. 
Journal of Controlled Release 2014, 190, 254-273.

79. Hoare, T. R.; Kohane, D. S., Hydrogels in drug delivery: Progress and challenges. Polymer 2008, 49 (8), 1993-2007.

80. Vermonden, T.; Censi, R.; Hennink, W. E., Hydrogels for protein delivery. Chemical Reviews 2012, 112 (5), 2853-2888.

81. Tibbitt, M. W.; Anseth, K. S., Hydrogels as extracellular matrix mimics for 3D cell culture. Biotechnology and Bioengineering 2009, 103 (4), 655-663.

82. Caliari, S. R.; Burdick, J. A., A practical guide to hydrogels for cell culture. Nature Methods 2016, 13 (5), 405-414.

83. Mirbagheri, M.; Adibnia, V.; Hughes, B. R.; Waldman, S. D.; Banquy, X.; Hwang, D. K., Advanced cell culture platforms: a growing quest for emulating natural tissues. Materials Horizons 2019, 6 (1), 45-71.

84. Zhang, Y.; Yu, J.; Ren, K.; Zuo, J.; Ding, J.; Chen, X., Thermosensitive Hydrogels as Scaffolds for Cartilage Tissue Engineering. Biomacromolecules 2019, 20 (4), 1478-1492.

85. Censi, R.; Schuurman, W.; Malda, J.; di Dato, G.; Burgisser, P. E.; Dhert, W. J. A.; van Nostrum, C. F.; di Martino, P.; Vermonden, T.; Hennink, W. E., A Printable Photopolymerizable Thermosensitive p(HPMAm-lactate)-PEG Hydrogel for Tissue Engineering. Advanced Functional Materials 2011, 21 (10), 1833-1842.

86. Patenaude, M.; Hoare, T., Injectable, Mixed Natural-Synthetic Polymer Hydrogels with Modular Properties. Biomacromolecules 2012, 13 (2), 369-378.

87. Bhattarai, N.; Gunn, J.; Zhang, M., Chitosan-based hydrogels for controlled, localized drug delivery. Advanced Drug Delivery Reviews 2010, 62 (1), 83-99.

88. de Almeida, P.; Jaspers, M.; Vaessen, S.; Tagit, O.; Portale, G.; Rowan, A. E.; Kouwer, P. H. J., Cytoskeletal stiffening in synthetic hydrogels. Nature Communications 2019, 10 (1), article 609, (page 1-8).

89. Hoffman, A. S., Stimuli-responsive polymers: Biomedical applications and challenges for clinical translation. Advanced Drug Delivery Reviews 2013, 65 (1), 10-16.

90. Soppimath, K. S.; Aminabhavi, T. M.; Dave, A. M.; Kumbar, S. G.; Rudzinski, W. E., Stimulus-Responsive "Smart" Hydrogels as Novel Drug Delivery Systems. Drug Development and Industrial Pharmacy 2002, 28 (8), 957-974.

91. Li, Z.; Guan, J., Thermosensitive hydrogels for drug delivery. Expert Opinion on Drug Delivery 2011, 8 (8), 991-1007.

92. Wang, X.; Wang, C.; Zhang, Q.; Cheng, Y., Near infrared light-responsive and injectable supramolecular hydrogels for on-demand drug delivery. Chemical Communications 2016, 52 (5), 978-981.

93. Katz, J. S.; Burdick, J. A., Light-Responsive Biomaterials: Development and Applications. Macromolecular Bioscience 2010, 10 (4), 339-348. 
94. Hu, J.; Zhang, G.; Liu, S., Enzyme-responsive polymeric assemblies, nanoparticles and hydrogels. Chemical Society Reviews 2012, 41 (18), 5933-5949.

95. Gupta, P.; Vermani, K.; Garg, S., Hydrogels: from controlled release to $\mathrm{pH}$-responsive drug delivery. Drug Discovery Today 2002, 7 (10), 569-579.

96. Raza, F.; Zhu, Y.; Chen, L.; You, X.; Zhang, J.; Khan, A.; Khan, M. W.; Hasnat, M.; Zafar, H.; $W$ u, J.; Ge, L., Paclitaxel-loaded $\mathrm{pH}$ responsive hydrogel based on selfassembled peptides for tumor targeting. Biomaterials Science 2019, 7 (5), 2023-2036.

97. Purcell, B. P.; Lobb, D.; Charati, M. B.; Dorsey, S. M.; Wade, R. J.; Zellars, K. N.; Doviak, H.; Pettaway, S.; Logdon, C. B.; Shuman, J. A.; Freels, P. D.; Gorman lii, J. H.; Gorman, R. C.; Spinale, F. G.; Burdick, J. A., Injectable and bioresponsive hydrogels for ondemand matrix metalloproteinase inhibition. Nature Materials 2014, 13, 653-661.

98. Weeks, E. R., Introduction to the Colloidal Glass Transition. ACS Macro Letters 2017, 6 (1), 27-34.

99. Trappe, V.; Weitz, D. A., Scaling of the Viscoelasticity of Weakly Attractive Particles. Physical Review Letters 2000, 85 (2), 449-452.

100. Kratz, K.; Hellweg, T.; Eimer, W., Influence of charge density on the swelling of colloidal poly( $\mathrm{N}$-isopropylacrylamide-co-acrylic acid) microgels. Colloids and Surfaces $A$ : Physicochemical and Engineering Aspects 2000, 170 (2), 137-149.

101. Romeo, G.; Fernandez-Nieves, A.; Wyss, H. M.; Acierno, D.; Weitz, D. A., Temperature-Controlled Transitions Between Glass, Liquid, and Gel States in Dense p-NIPA Suspensions. Advanced Materials 2010, 22 (31), 3441-3445.

102. Heskins, M.; Guillet, J. E., Solution Properties of Poly(N-isopropylacrylamide). Journal of Macromolecular Science: Part A - Chemistry 1968, 2 (8), 1441-1455.

103. Wu, C.; Zhou, S.; Au-yeung, S. C. F.; Jiang, S., Volume phase transition of spherical microgel particles. Die Angewandte Makromolekulare Chemie 1996, 240 (1), 123-136.

104. Minami, S.; Suzuki, D.; Urayama, K., Rheological aspects of colloidal gels in thermoresponsive microgel suspensions: formation, structure, and linear and nonlinear viscoelasticity. Current Opinion in Colloid \& Interface Science 2019, 43, 113-124.

105. Wang, T.; Jin, L.; Song, Y.; Li, J.; Gao, Y.; Shi, S., Rheological study on the thermoinduced gelation behavior of poly( $\mathrm{N}$-isopropylacrylamide-co-acrylic acid) microgel suspensions. Journal of Applied Polymer Science 2017, 134 (35), article 45259, (page 1-8).

106. Minami, S.; Watanabe, T.; Suzuki, D.; Urayama, K., Viscoelasticity of dense suspensions of thermosensitive microgel mixtures undergoing colloidal gelation. Soft Matter 2018, 14 (9), 1596-1607. 



\section{Chapter 2}

\section{Poly( $N$-isopropylacrylamide); physicochemical properties and biomedical applications}

Marzieh Najafi, Erik Hebels, Wim E. Hennink, Tina Vermonden

Department of Pharmaceutics, Utrecht Institute for Pharmaceutical Sciences (UIPS), Faculty of Science, Utrecht University, P.O. Box 80082, 3508 TB Utrecht, The Netherlands.

Book chapter, Temperature-Responsive Polymers: Chemistry, Properties, and Applications, John Wiley \& Sons 2018; pp 1-34. 


\section{Introduction}

Poly(N-isopropylacrylamide) (PNIPAM, Figure 1) has attracted a lot of attention during the past decades because of its thermoresponsive behaviour in a biomedically interesting temperature window. This polymer exhibits inverse solubility in aqueous media and precipitates upon increasing the temperature. ${ }^{1,2}$ The temperature at which this polymer converts from a soluble state to an insoluble state, known as the cloud point (CP) or the lower critical transition temperature (LCST), is $32{ }^{\circ} \mathrm{C} .{ }^{3}$ The first study on the PNIPAM phase diagram was reported by Heskins et al. ${ }^{2}$ Since then this polymer has been known as a thermosensitive polymer. PNIPAM has been prepared by a wide range of polymerization techniques such as free radical polymerization, ${ }^{4}$ redox polymerization, ${ }^{5}$ ionic polymerization, ${ }^{6}$ radiation polymerization, ${ }^{7}$ and living radical polymerization. ${ }^{8}$

The focus of this chapter is on polymerization techniques and examples are given addressing PNIPAM's potential applications as biomaterial in drug and gene delivery, and bioseparation. For other applications of PNIPAM in e.g. membranes, sensors, thin films and brushes, the reader is refered to reviews published elsewhere. ${ }^{9,10,11,12}$

After introducing the general physicochemical properties of PNIPAM, an overview of the most frequently used polymerization techniques (free and living radical polymerization) is given and a variety of copolymers and structures obtained by these methods is highlighted. Copolymerization with other monomers or conjugation/grafting of PNIPAM with other stimuli-responsive polymers/materials results in dual responsive materials, of which the physical properties can be changed by several stimuli e.g. changes in $\mathrm{pH}$ or redox conditions, light and magnetic field. Examples of these systems along with the effect of copolymer composition on the LCST of PNIPAM are provided in this chapter. In addition, different methods of chemical and physical crosslinking and their effects on properties of the final materials are discussed.

Also, the potential of designing complex bioconjugates provided by recent developments in polymerization methods is discussed. Conjugation of responsive polymers to biomolecules (e.g. proteins, peptides, and nucleic acids) is a sophisticated method because the attached PNIPAM imparts responsiveness to these biomolecules. Furthermore, conjugation to biomolecules induces changes in stability and bioactivity as a result of altering the (surface) properties and solubility of materials. Here, we will review examples of grafting PNIPAM to biomolecules or growing polymeric chains from their surfaces. Finally, the future prospects of PNIPAM in biomedical and pharmaceutical applications are outlined. 


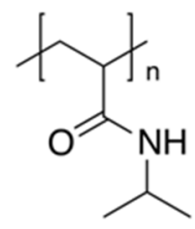

Figure 1. Chemical structure of poly(N-isopropyl acrylamide) (PNIPAM)

\section{PNIPAM as thermosensitive polymer}

Thermosensitive polymers are by definition polymers whose physical properties can change in response to temperature changes, usually occurring in aqueous media. ${ }^{13}$ This transition is most often drastic and follows upon passing a certain threshold which may be, in context of miscibility in a solvent, either an upper or lower critical solution temperature (UCST and LCST). LCST behaviour indicates the temperature above which the polymer will no longer be soluble whilst UCST behavior indicates the temperature below which immiscibility is reached. It should be noted that in literature the terms cloud point (CP) and LCST are often mixed up. The $\mathrm{CP}$ of a polymer solvent mixture is the temperature at which separation into a polymer rich and polymer poor phase occurs. The LCST is defined as the minimum of the CP in a temperature versus polymer concentration plot. So by definition, below the LCST, only one phase is observed independent of the polymer concentration (see section 3). ${ }^{14}$ PNIPAM is an especially interesting thermosensitive polymer for application in biomedical and pharmaceutical sciences because of its sharp lower critical solution temperature of $32{ }^{\circ} \mathrm{C}$ in aqueous media. This transition is reversible and PNIPAM solubilizes again when the temperature drops below its LCST. ${ }^{3}$

The exact mechanism by which PNIPAM self-assembles in water above the LCST is still not fully clear but believed to be because of the entropic gain of water molecules that dissociate from the hydrophobic isopropyl side chain moieties above the LCST. The enthalpy gain of water molecules associated via hydrogen bonds with the amide groups of the polymer becomes smaller than the counter effect of entropic gain of the system with water being dissociated when passing the LCST. ${ }^{3}$ Since the extent of hydration of polymers is dependent on the characteristics of the monomer units, the LCST of PNIPAM may be varied by copolymerizing NIPAM with monomers differing in hydrophobicity or hydrophilicity. Furthermore, hydrophobic interactions between the polymer segments themselves have also been suggested to be crucial to the LCST transition from isolated extended coils of PNIPAM to collapsed chains. $3,15,16$

Water molecules form hydrogen bonds with the carbonyl group, accepting two hydrogen 
bonds, and the nitrogen atom of the amide group can donate one hydrogen bond in the hydrated state below LCST. ${ }^{16}$ During this transition, it has been shown that the number of hydrogen bonds between PNIPAM and water are reduced and intra-chain hydrogen bonds are formed instead of which some remain, even when cooled again below LCST. This explanation is used to rationalize why the aggregated chains swell upon cooling and do not immediately dissociate slightly below the LCST and hence cause hysteretic behavior. ${ }^{17}$ Computer simulations confirmed that besides a reduction of intermolecular hydrogen bonds, there is a substantial decrease in the solvent accessible surface area and it has been even suggested that a decrease in torsional energy of the isopropyl groups occurs during this thermal transition. The model also predicted the decrease in LCST upon copolymerizing with hydrophobic tBAAM (tert-butylacrylamide), which is in line with experimental results. ${ }^{18}$ The carbon backbone has shown to play an important role in the hydrophobic contribution of phase transition. To investigate this effect, Lai et al ${ }^{19}$ used $\mathrm{N}$-isopropylpropionamide (NIPPA) as a small molecular model compound for PNIPAM. They observed that at high concentration (40 wt\%), the NIPPA solution shows a higher LCST of $39{ }^{\circ} \mathrm{C}$ with a broader phase transition temperature range. They explained that the carbonyl group in the small molecule of NIPPA has more interaction with water molecules, which explains the higher LCST. Yet, the presence of the hydrophobic main chain in PNIPAM interferes with hydrogen bonding between the carbonyl groups and water molecules. ${ }^{19}$ On the other hand, the presence of $\alpha$-methyl groups in the main chain (poly( $N$-isopropylmethacrylamide), PNIPMAM) results in increased hydrophobicity, however, surprisingly the LCST of this polymer is not lower than that of PNIPAM but even increased by about $15^{\circ} \mathrm{C}$. The authors speculated that the higher CP for PNIPMAM is due to the methyl groups that induce sterical hindrance for the hydrophobic groups to self-assemble in the most favorable manner. ${ }^{20}$

\section{Physical properties of PNIPAM}

This section briefly describes some of the physical properties of PNIPAM by highlighting the effect of composition of the media on its phase transition temperature.

\subsection{Phase behavior of PNIPAM in water/alcohol mixtures}


In water/organic solvent mixtures (e.g. alcohols/acetone) the LCST of PNIPAM is dependent on the type of co-solvent and its volume fraction. In general, first a decrease in a cloud point is found upon increasing the volume fraction of organic solvent, while after a certain volume ratio an increase in a cloud point is observed. The less polar the co-solvent, the lower the volume fraction at which the increase in the transition temperature occurs. For example, for acetone the minimum transition temperature is found at a molar

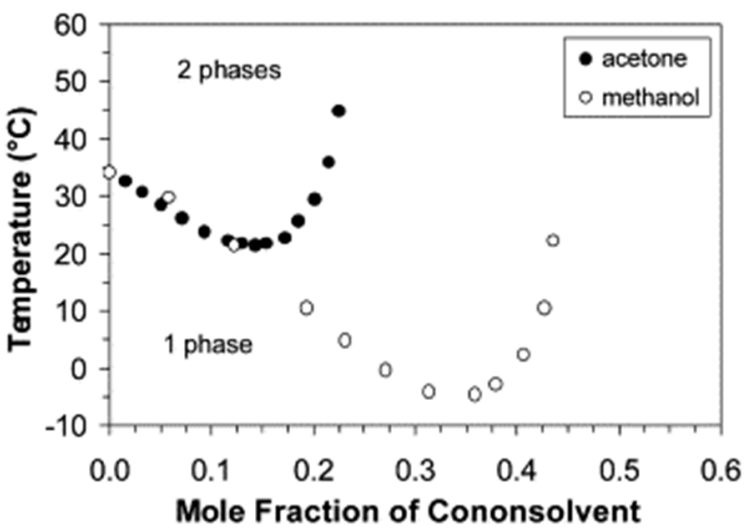

Figure 2: Comparison between phase transition temperatures of PNIPAM in water-methanol (open symbols) and water-acetone (filled symbols) solutions. Reported with permission from Ref. ${ }^{22}$

fraction of 0.15 , while for methanol this mole fraction is 0.34 (Figure 2). At low volume ratios, the co-solvent molecules and PNIPAM compete for water molecules, resulting in less hydration of PNIPAM and thus a lower cloud point. Upon increasing the volume fraction of a co-solvent, these solvent molecules interact with the polymer chains and increase their solubility. Remarkably, for some alcoholic co-solvents such as ethanol and 1-propanol a coexistence of LCST and UCST behavior is observed. In contrast, UCST behavior is not observed in water only or methanol-water mixtures. ${ }^{15,21,22}$

\subsection{Effect of concentration and molecular weight of PNIPAM on LCST}

As mentioned before (see section 2), the cloud point of a solution of PNIPAM versus the polymer concentration in the solvent can be used to establish the LCST. Previously, it has only been possible to investigate up to $40 \%$ weight concentration of PNIPAM in water due to the high viscosity of high concentration polymer solutions resulting in loading difficulties of glass capillaries. By use of nanoliter microchambers and microevaporation it is possible to concentrate PNIPAM in water up to $60 \%$ by weight and still be able to measure cloud points to establish the phase diagram of water/PNIPAM systems..$^{23,24,25}$

A decrease in CP can clearly be seen as concentration is increased to $40 \mathrm{wt} \%$, which is in agreement with previous studies conducted. ${ }^{26,27}$ Above 40 wt\%, the CP increases (Figure 3). It should be noted that although a wide concentration range was investigated, the LCST varies very little (between 28.5 and $32{ }^{\circ} \mathrm{C}$ ). 
There are various reports about the effect of molecular weight on the LCST of PNIPAM. According to these papers, the LCST could increase $^{28}$ or decrease $e^{29,30}$ or remain almost unchanged ${ }^{31,32 \text {, }}$ upon increasing the molecular weight of PNIPAM. The reasons for these different observations are not clear but may be related to differences in the concentrations used, measuring techniques, and variations in polymer end groups. Furyk et al. ${ }^{25}$ observed that the effect of end groups on LCST of PNIPAM is significant when the molecular weight is below $50 \mathrm{kDa}$. In general for these relatively

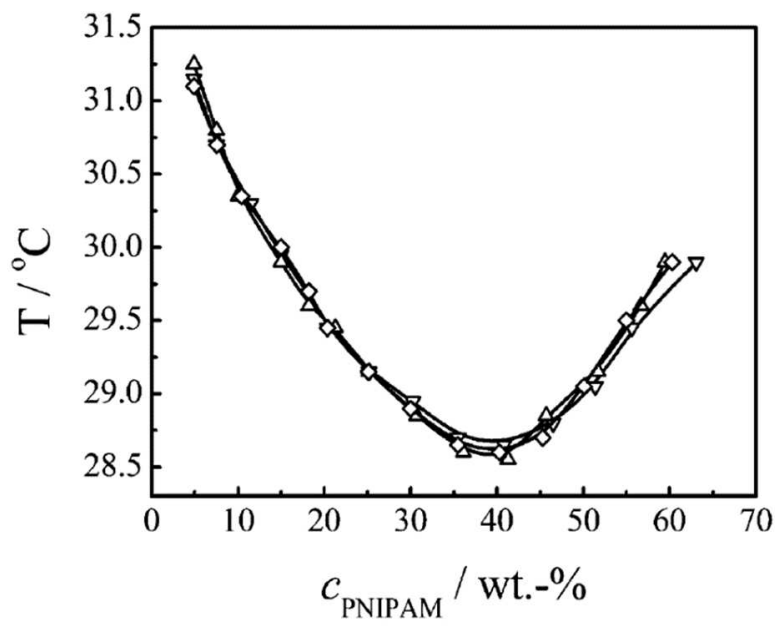

Figure 3: Phase diagram showing the concentration dependence of the cloud point temperatures of PNIPAM, $M_{w}=3.9 \times 10^{5}$ dissolved in water. The three curves are from three parallel measurements. Reported with permission from Ref. ${ }^{202}$ small polymers, the presence of hydrophilic or hydrophobic end groups results in higher or lower LCST, respectively. ${ }^{14,25}$

\subsection{Effect of surfactants on LCST}

Ionic surfactants such as sodium dodecyl sulfate (SDS) increase the LCST of PNIPAM by binding to the polymer and thereby converting it into a polyelectrolyte in a concentration dependent manner. This is caused by the entropy of counterions, which favors polymer hydration. ${ }^{33,34}$ The result of this can be seen in Figure 4. Here the LCST is found by measuring solution viscosity rather than the usually employed

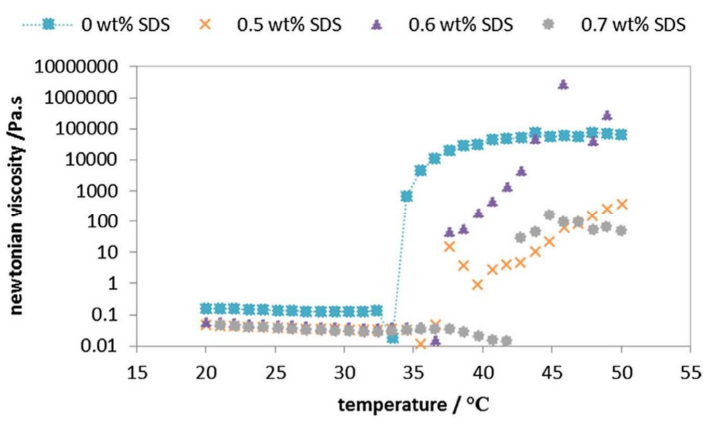

Figure 4: Dependence of Newtonian viscosity on temperature (heating system). Effect of the addition of SDS to 5 wt.\% PNIPAM (39 k) solutions. The depicted lines are methods determining the cloud point by a guide to the eye. Reported with permission from Ref. ${ }^{33}$ light scattering. 


\subsection{Effect of salts on LCST}

Salts can be classified either as kosmotropes or chaotropes depending on their ability to either salt-out or salt-in proteins/macromolecules in aqueous solutions as defined by the Hofmeister series. Kosmotropes (such as $\mathrm{CO}_{3}{ }^{2-}$, $\mathrm{SO}_{4}{ }^{2-}$ and $\mathrm{HPO}_{4}{ }^{2-}$ ) are strongly hydrated and have stabilizing and salting-out effects on proteins and macromolecules dissolved in water, whilst chaotropes (such as $\mathrm{SCN}^{-}, \mathrm{ClO}_{4}^{-}$ and $\left.\mathrm{I}^{-}\right)$destabilize folded $\mathrm{F}^{-} \approx \mathrm{SO}_{4}^{2-}>\mathrm{HPO}_{4}^{2-}>$ acetate $>\mathrm{Cl}^{-}>\mathrm{NO}_{3}^{-}>\mathrm{Br}^{-}>\mathrm{ClO}_{3}^{-}>\mathrm{I}^{-}>\mathrm{ClO}_{4}^{-}>\mathrm{SCN}^{-}$

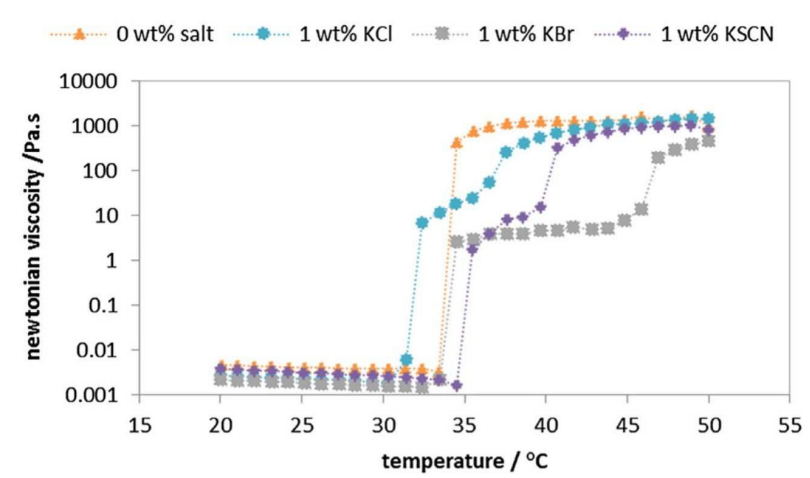

Figure 5: Dependence of Newtonian viscosity on temperature (heating system). Effect of anions in 5 wt. \% PNIPAM (20 k) solutions. The depicted lines are a guide to the eyes. Reported with permission from Ref. ${ }^{33}$ The Hofmeister series is depicted above the figure.

\section{proteins resulting in so-called} salting-in behavior. ${ }^{35}$ Indeed this effect can be seen experimentally for PNIPAM as well (Figure 5). Importantly, KBr does not alter the LCST (marking the middle of the Hofmeister series) whilst $\mathrm{KCl}$ (kosmotrope) and $\mathrm{KSCN}$ (chaotrope) have opposite effects on LCST. ${ }^{33}$ Interestingly, a second transition can be observed upon addition of salt which is attributed to the separate dehydration of isopropyl groups. ${ }^{33,35,36}$

\section{Common methods for polymerization of NIPAM}

\subsection{Free radical polymerization}

Free radical polymerization (FRP) is a standard and facile method for the polymerization of a great variety of monomers with unsaturated carbon-carbon bonds. It starts with an initiator molecule that is thermally or by UV-radiation decomposed into free radical(s) and that subsequently react(s) with monomers bearing carbon-carbon double bonds such as vinyl, acrylate or methacrylate groups. ${ }^{37}$ In the next step, radical containing moieties react with a next monomer molecule, resulting in chain propagation until termination of chain growth occurs when two free radical containing molecules react with each other. Poly $(N$ isopropylacrylamide) (PNIPAM) was first synthesized in the 1950 s by this conventional 
polymerization method. ${ }^{3}$ Although the molecular weight distribution obtained using FRP is rather broad, it is up to today a very versatile and frequently used polymerization technique, which can be performed in different organic solvents as well as in aqueous media. Common organic solvents used for this polymerization are methanol, benzene, acetone, THF, $t$ butanol, dioxane, chloroform as well as mixtures of these solvents. ${ }^{37}$ Typically, the preferred initiator is azobisisobutyronitrile (AIBN) although also other initiators have been used. Polymeric chains can inherit chain ends from the initiator depending on the type of initiator used. For instance, England et al. ${ }^{38}$ used 1-phenyl(trimethylsiloxy) ethylene as an initiator for polymerization of NIPAM and in this way obtained phenyl derivatized PNIPAM. ${ }^{38}$

As discussed in sections 2 and 3, a sharp transition in hydrophilicity of PNIPAM in response to temperature close to physiological temperature makes this polymer interesting for bioapplications. ${ }^{39}$ Another important criterion for using a polymer in biomedical applications is biodegradability, and PNIPAM, as a non-resorbable polymer, faces a challenge in this regard. A way of solving this problem is free radical copolymerization of NIPAM with hydrolysable monomers, such as $\mathrm{N}$-(2-hydroxypropyl)-methacrylamide lactate (HPMAmlactate $)^{40}$ or dimethyl- $y$-butyrolactone acrylate (DBA ${ }^{41}$. Hydrolysis of these groups increases the overall hydrophilicity of the copolymer which in turn results in an increase of the LCST. When the LCST passes body temperature, the polymer becomes soluble in body fluids and can potentially be secreted by the kidneys when the molecular weight is below a threshold of $45 \mathrm{kDa} .{ }^{42}$ Shah et al. ${ }^{43}$ copolymerized NIPAM with $\mathrm{N}$-acryloxysuccinimide (NAS) to modulate the biodegradability of PNIPAM. They showed an increase in the LCST of the polymer after hydrolysis of the NHS groups, which resulted in the formation of polar carboxyl moieties in the polymer chains. Similarly, the kinetics of polymer degradation could also be modulated by conjugation of a hydrophobic drug to the polymer via a hydrolysable linker. Hence, hydrolysis of the drug linker not only results in drug release, but also increases hydrophilicity and solubility of the polymer at body temperature as a result of increase in the LCST of polymer. ${ }^{43}$ Our group ${ }^{44}$ copolymerized NIPAM with HPMAm-mono(di)lactate monomers starting from a PEG-initiator to obtain P(NIPAM-co-HPMAm-mono(di)-lactate)$b$-PEG. Hydrolysis of the lactate ester side groups under physiological conditions in time led to an increase in hydrophilicity of the polymer, which resulted in a gradual increase in a cloud point. ${ }^{40}$ In another study, we showed that the block copolymer of P(NIPAM-coHPMAm-di-lactate)- $b$-PEG forms nanoparticles with a size of $\sim 70 \mathrm{~nm}$ above the LCST of the thermosensitive segment in water. $^{45}$

Apart from these examples, many studies have been devoted to free radical polymerization and copolymerization of NIPAM to obtain polymeric structures with interesting features for bioapplications..$^{46,47,48,49}$ For instance, Topp et al. ${ }^{50}$ combined the hydrophobic nature of 
PNIPAM above its LCST with the hydrophilic character of PEG in a block copolymer to obtain thermosensitive micelles.

\subsection{Living radical polymerization}

Living radical polymerizations are versatile techniques to control polymer molecular weight, architecture and copolymer composition. This control is based on the fact that the lifetime of a radical in the propagating step is longer than the lifetime of a radical during conventional free radical polymerization. Generally, living polymerization is characterized by a fast initiation and slow propagation and absence of termination. ${ }^{51}$ The increase in the lifetime is due to a reversible equilibrium between the active species $\left(\mathrm{P}^{*}\right)$ and dormant species $(\mathrm{P}-\mathrm{X})$, which minimizes irreversible chain stopping events e.g. termination (Figure 6).

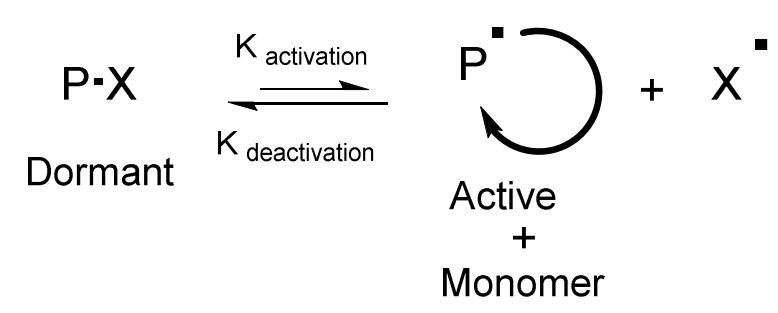

Figure 6: Reversible activation process in living radical polymerization.

Several methods have been developed for living radical polymerization such as nitroxide mediated polymerization (NMP), ${ }^{52,53}$ (reverse) iodine transfer polymerization (ITP and RITP), ${ }^{54}$ single electron transfer-degenerative transfer living radical polymerization (SETDTLRP), ${ }^{55}$ reversible addition fragmentation chain transfer (RAFT), ${ }^{56}$ atom transfer radical polymerization (ATRP) ${ }^{57}$ and single electron transfer-living radical polymerization (SETLRP). ${ }^{58}$ In this section, we focus on living radical polymerization of NIPAM using ATRP and RAFT as these methods are most commonly used and studied for polymerization of this monomer.

\subsubsection{ATRP polymerization of NIPAM}

Atom Transfer Radical Polymerization (ATRP) ${ }^{57}$ is a polymerization technique that offers good control over polymer molecular weight and polymer design. ATRP of a wide range of monomers (including NIPAM) can be carried out in both organic solvents and in aqueous media. ${ }^{59}$ NIPAM has been copolymerized with different hydrophilic and hydrophobic 
monomers by ATRP to yield polymers for a wide range of applications. ${ }^{46,60}$ However, ATRP polymerization of acrylamides can be problematic because of complexation of the amide group to the copper catalyst, which can lead to catalyst deactivation. The solvent choice therefore plays a key role in successful polymerization of this class of monomers. It is known that polar protic solvents form hydrogen-bonds with both monomers and polymers, thereby reducing the possibility of monomer complexation with the copper catalyst. ${ }^{61}$ However, the use of a protic solvent increases the risk of fast and less controlled polymerization due to inefficient deactivation, relative to activation and propagation in ATRP polymerization. ${ }^{62}$ A successful example of living radical copolymerization of NIPAM in water was reported by Haddleton et al. ${ }^{63}$ They copolymerized NIPAM with different watersoluble monomers like $\mathrm{N}, \mathrm{N}$-dimethyl acrylamide (DMA), 2-hydroxyethyl acrylate (HEA) and oligo(ethylene oxide) acrylate (OEOA), and obtained polymers with narrow molecular weight distributions (PDI 1.1). The authors also showed that the bromine chain-ends remain intact during polymerization, ${ }^{63}$ which provides the possibility to synthesize di- and even multiblock copolymers.

As discussed before, the cloud point (see section 2) can be tuned by incorporation of different hydrophobic and hydrophilic monomers in NIPAM copolymers and this strategy has also been used for polymers prepared by ATRP. For example, incorporation of $N, N$ dimethylacrylamide (DMA) into PNIPAM changes the LCST to a temperature slightly higher than body temperature $\left(37^{\circ} \mathrm{C}\right)$ as a result of the hydrophilic nature of DMA. Hu et al..$^{64}$ synthesized a triblock copolymer of P(NIPAM-co-DMA)- $b$-PLLA- $b$-P(NIPAM-co-DMA) by ATRP using Br-PLLA-Br as macroinitiator. They demonstrated that by increasing the ratio of DMA/NIPAM from 0 to $24 \%$, the LCST linearly increased from 32.2 to $39.1{ }^{\circ} \mathrm{C} .{ }^{64}$ This system was able to self-assemble in aqueous medium into micelles below the LCST because of the hydrophobic PLLA domains of this tri-block copolymer. ${ }^{64}$ Li et al. ${ }^{65}$ applied ATRP polymerization for the synthesis of a hydrogel based on NIPAM, DBA, and 2-hydroxyethyl methacrylate (HEMA) using a polycaprolactone macroinitiator. Physical properties of resulting hydrogel along with supporting cardiosphere-derived cells (CDCs) proliferation made this system a suitable candidate for myocardial injection of CDCs for cardiac cell therapy. The hydrolysis of the ester bonds in the lactone ring of the DBA moieties resulted in a gradual increase in LCST (above $37^{\circ} \mathrm{C}$ ) which led to solubility of the resulting polymer in body fluids over time. ${ }^{65}$

De Graaf et al. ${ }^{66}$ used ATRP polymerization for the synthesis of AB diblock and BAB triblock copolymers (block $A$ is poly(ethylene glycol) (PEG) and block $B$ is PNIPAM). These polymers once dissolved in water and at low concentration formed star-like and flower-like micelles. ${ }^{66}$ On the other hand, solutions of BAB polymers at high polymer concentrations formed 
hydrogels above the LCST of the polymer. These hydrogels were loaded with paclitaxel (PTX) and it was shown that they released drug-loaded flower-like micelles when in contact with an aqueous environment. An in vivo study in mice showed a reduced tumor growth using these hydrogel formulations upon intraperitoneal injection. ${ }^{67}$

Kim et al. ${ }^{68}$ used a PEG macroinitiator for ATRP polymerization of NIPAM at $25^{\circ} \mathrm{C}$ in aqueous media. They introduced $N, N^{\prime}$-ethylenebisacrylamide during polymerization to prepare stable hydrogel nanoparticles and controlled the size of these nanoparticles (from 300$1200 \mathrm{~nm}$ ) by using THF as a co-solvent. THF increases the solubility of growing PNIPAM chains, which could explain the increase in size of the nanoparticles in the presence of this solvent. $^{68}$

ATRP also is a powerful method for graft polymerization. For instance, Gin et al. ${ }^{69}$ reported graft polymerization of PNIPAM onto poly( $N$-vinylpyrrolidone) (PVP) by ATRP. After free radical polymerization of $P V P$, the pendant allylic groups of the obtained polymer were functionalized with $N$-bromosuccinimide (NBS) to form PVP-Br ATRP initiators. Subsequently, brushes of PNIPAM were grafted from PVP in the presence of $\mathrm{CuCl}$ and bipyridine as a catalyst in water and at room temperature. An aqueous solution of PVP-g-PNIPAM was converted into hydrogel above the cloud point of the polymer at $35.3^{\circ} \mathrm{C}$. This system has potential applications in drug delivery as it was shown that the kinetics of drug release was controlled by drug diffusion through the gel. ${ }^{69}$

\subsubsection{RAFT polymerization of NIPAM}

Reversible addition-fragmentation chain transfer (RAFT) ${ }^{56}$ polymerization is another versatile technique used to polymerize a wide range of monomers. Different from ATRP, RAFT polymerization proceeds without the need for a metal catalyst, but requires the presence of a radical initiator e.g. azobisisobutyronitrile (AIBN) and a RAFT chain transfer agent (CTA). The RAFT agent consists of a thiocarbonylthio moiety and a so-called R and $Z$ group (Figure 7). The $Z$ group primarily affects the stability of the $S=C$ bond and the stability of the adduct radical while the R group initiates growth of a polymer chain. ${ }^{70,71}$<smiles>[R]SC([Z])=S</smiles>

Figure 7: General structure of the RAFT chain transfer agent.

RAFT polymerization can be performed in both organic and aqueous solvents as well as their mixtures. The first example of RAFT polymerization of NIPAM in water at ambient temperature was reported by McCormick et al. ${ }^{72}$ using mono- and difunctional $\mathrm{N}, \mathrm{N}$-dimethyl 
acrylamide (DMA) macro-CTA's. Diblock $(A B)$ and triblock copolymers (BAB) were obtained using a fixed molecular weight DMA macro-CTA as A block and varying molecular weights of PNIPAM as B block(s) with narrow PDI (PDI $\approx 1.15)$. Micellization of these polymers occurred above the $\mathrm{CP}$ of the polymers $\left(34-45^{\circ} \mathrm{C}\right)$ with longer PNIPAM chains, as expected, showing a lower LCST. However, triblock polymers with short PNIPAM blocks did not form micelles at any temperature. ${ }^{72}$

A RAFT agent can be immobilized onto a surface or substrate and can be used to introduce different functional groups after polymerization. Polymer growth from, for example, a protein provides the possibility to synthesize polymer-bioconjugates. Conversion of the RAFT agent after polymerization includes, for instance, hydrolysis of thiocarbonylthio group resulting in a free thiol that can be subsequently used for thiol-ene, ${ }^{73}$ thiol-isocyanate ${ }^{74}$ click reactions or to form a reduction sensitive disulfide bond. ${ }^{75}$

\section{Dual sensitive systems}

\section{1. pH and thermosensitive systems}

The $\mathrm{pH}$ of various tissues and cellular compartments differs, for example, the $\mathrm{pH}$ of blood is 7.4, whereas in the stomach the $\mathrm{pH}$ ranges from 1.0-5.0. It has also been reported that the $\mathrm{pH}$ in tumors and other sites of inflammation can be as low as 6.5-6.9. ${ }^{76,77}$ Finally, the $\mathrm{pH}$ in cellular vesicles like endosomes and lysosomes (the compartments in which e.g. nanomedicines mostly accumulate after internalization ${ }^{78,79}$ ) can be between 5.0 and 6.2..$^{80,81}$ Polymers containing monomers that can alter their ionization states upon variation of the $\mathrm{pH}$ are interesting for the design of triggerable drug delivery systems. ${ }^{82}$ Commonly used monomers in $\mathrm{pH}$ responsive polymers are acrylic acid (AA), methacrylic acid (MAA), and $N, N$ dimethylaminoethyl methacrylate (DMAEMA). Also maleic anhydride (MA) is frequently used, which after hydrolysis leaves carboxylic acid moieties on the polymer chain.

An example of a thermo- and $\mathrm{pH}$ - responsive diblock copolymer was reported by Chang et al. ${ }^{83}$ They synthesized a PNIPAM-poly(lysine) diblock polymer using a heterofunctional initiator designed for ATRP polymerization of NIPAM and ring opening polymerization of $\mathrm{N}^{\varepsilon}$ (carbobenzoxy)-L-lysine-N-carboxyanhydride (Z-L-lysine NCA). The heterofunctional initiator had a phthalimido moiety on one side and an ATRP initiator on the other side. In short, first ATRP polymerization of NIPAM was performed using $\mathrm{CuBr} / \mathrm{Me}_{6}$ TREN in 2propanol at $0{ }^{\circ} \mathrm{C}$. Subsequently, hydrolysis of the phthalimido group on the other terminal end of the polymeric chain resulted in a primary amine-functionalized PNIPAM (PNIPAM$\mathrm{NH}_{2}$ ), which was used as macroinitiator for ring opening polymerization of Z-L-lysine NCA in 
DMF at $20^{\circ} \mathrm{C}$ to obtain poly( $N$-isopropylacrylamide)- $b$-poly(Z-L-lysine) (PNIPAM- $b$-PZLys). This amphiphilic block copolymer can undergo coil-to-helix and coil-globule transitions as a response to changes in $\mathrm{pH}$ and temperature. ${ }^{83}$

Chen et al. ${ }^{84}$ combined ATRP and RAFT polymerization to synthesize linear tetrablock quaterpolymers consisting of PEG, poly(styrene) (PS), PNIPAM, and poly(2-(dimethylamino) ethyl methacrylate) (PDMAEMA) blocks. After ATRP polymerization of PS using a PEG macroinitiator, the resulting end-chain bromine groups were substituted by azide groups using $\mathrm{NaN}_{3}$. Subsequently, a click reaction between an alkyne-functionalized CTA and the azide group resulted in a PEG- $b$-PS-CTA RAFT macroinitiator. Polymerization continued by block copolymerization of NIPAM and DMAEMA and finally a PEG- $b$-PS- $b$-PNIPAM- $b$ PDMAEMA polymer was obtained and characterized. GPC analysis showed that the $M_{w}$ was $28 \mathrm{kDa}$ with a PDI of 1.3, which shows that multiblock copolymers with low PDI can be obtained by combining ATRP and RAFT. These linear polymers formed micelles with a PS core and PEG, PNIPAM and PDMAEMA as a shell at $\mathrm{pH} 4$ and $25^{\circ} \mathrm{C}$. Upon increasing the $\mathrm{pH}$ to 9, PDMAEMA blocks are deprotonated and the resulting more hydrophobic block participates in the core to yield micelles with PS/PDMAEMA hybrid core and a PEG/PNIPAM shell. On the other hand, at temperatures above the polymer LCST $\left(45^{\circ} \mathrm{C}\right)$ and at $\mathrm{pH} 4$, micelles with a PS/PNIPAM core and PEG/PDMAEMA shell were obtained. ${ }^{84}$

\subsection{Reduction sensitive and thermosensitive systems}

Polymeric structures containing disulfide bonds are interesting systems for drug delivery as reduction sensitive materials. Such polymers have shown potential for the design of nanoparticles suitable for intracellular delivery of drugs and other pharmacologically active compounds (like pharmaceutical proteins and nucleic acid based drugs). These systems are destabilized due to the significantly higher concentration of glutathione as reductive agent intracellularly resulting in release of the payload. ${ }^{85,86,87}$ A NIPAM based system was reported by Vogt et al. ${ }^{88}$ They first polymerized NIPAM using a difunctional trithiocarbonate and subsequently polymerized poly $(\mathrm{N}, \mathrm{N}$-dimethylacrylamide) (DMA) to obtain a reduction- and thermo-responsive PNIPAM- $b$-PDMA- $b$-PNIPAM polymer (Figure 8). They observed that below the LCST the polymer was fully soluble in aqueous solution and above the LCST, $40^{\circ} \mathrm{C}$, the polymer solution was converted into a hydrogel of micellar structures. In this network, the PNIPAM blocks form hydrophobic domains that were bridged by the hydrophilic PDMA blocks. The use of a bifunctional trithiocarbonate as initiator resulted in the presence of a cleavable trithiocarbonate linkage in the middle of the central pDMA block. At a polymer concentration of $50 \%$ a stable gel was formed. Aminolysis of the trithiocarbonate links 
resulted in the formation of PNIPAM- $b$-PDMA-SH, and consequently, gel destruction due to scission of the PDMA blocks responsible for intermicellar bridging. It was also demonstrated that subsequent oxidation of the thiol groups resulted in the formation of disulfide bridges and recovery of the gel (Figure 8). ${ }^{88}$

Another example of a thermally and biochemically responsive hydrogel based on NIPAM was reported by Li et al. ${ }^{89} \mathrm{~A}$ bifunctional ATRP initiator containing a disulfide bond was used for polymerization of 2-methacryloyloxyethyl phosphorylcholine (MPC) as a mid-block. Subsequently, NIPAM was polymerized using this macroinitiator to form a BAB triblock copolymer. Thermogelation resulted in a three- dimensional network, which was used to release hydrophobic anticancer drugs. Micelles can be released from this hydrogel in the presence of reducing agents like DTT or glutathione. ${ }^{89}$ However, at the moment the application of this hydrogel in vivo due to the absence of reductive agents in extracellular environments is questionable.

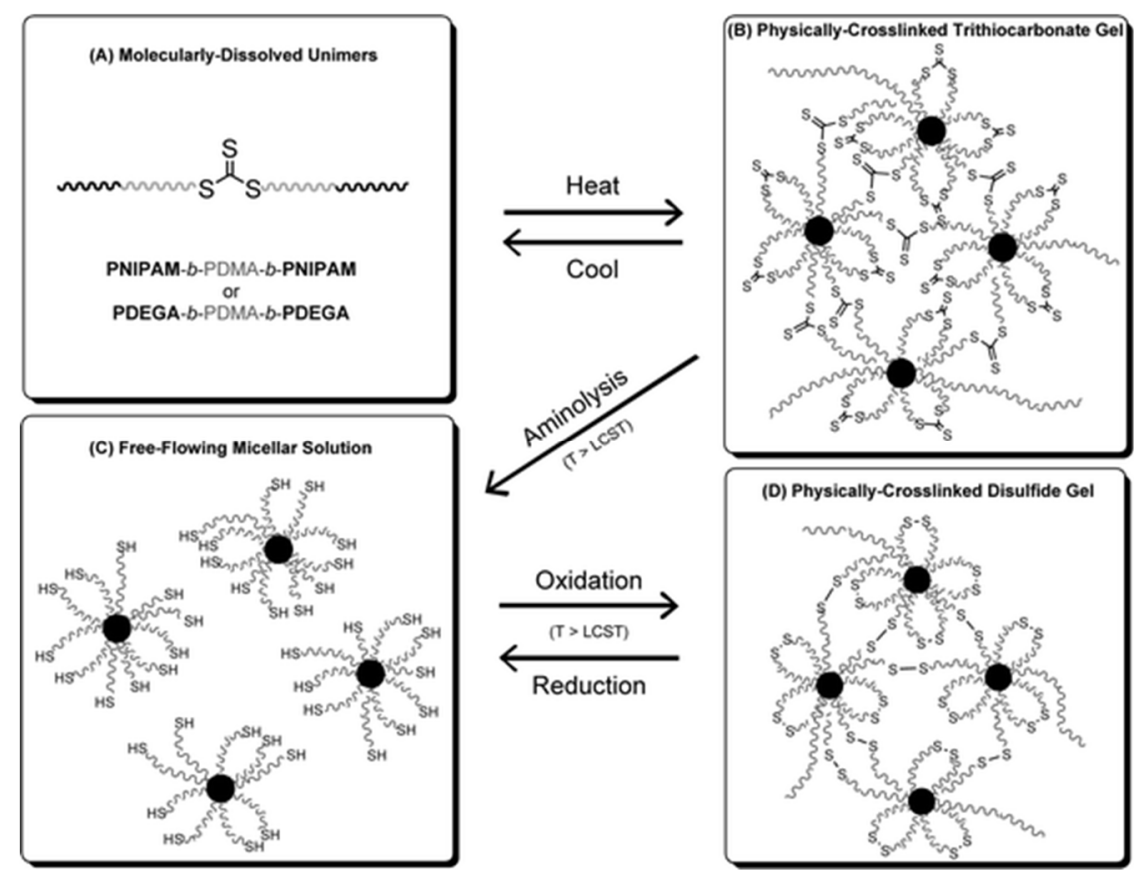

Figure 8: Temperature and redox-responsive gelation of triblock copolymers prepared by RAFT. (a) Molecularlydissolved unimers of PNIPAM-b-PDMA-b-PNIPAM or PDEGA-b-PDMA-b-PDEGA; (b) hydrogels are formed upon heating above the LCST of the responsive PNIPAM or PDEGA blocks; (c) free-flowing micellar solutions of PNIPAMb-PDMA-SH or PDEGA-b-PDMA-SH resulting from trithiocarbonate aminolysis at T > LCST; (d) hydrogels formed from PNIPAM-b-PDMA-S-S-PDMA -b-PNIPAM or PDEGA-b-PDMA-S-S-PDMA-b-PDEGA upon oxidation of the thiolterminated diblock aminolysis products. Reproduced with permission from Ref. ${ }^{88}$ 


\subsection{Hybrid thermosensitive materials}

Incorporation of metal particles into various PNIPAM based systems results in new classes of hybrid materials with attractive thermal, optical and magnetic properties. ${ }^{90,91}$ Different methods have been described for the encapsulation of metal nanoparticles into PNIPAM (micro/nano)gels ${ }^{92}$ or in situ formation of metal nanoparticles in the presence of PNIPAM. ${ }^{93,94}$ PNIPAM has also been directly grafted on the surface of metal nanoparticles. ${ }^{95,96}$

An example of in situ formation of gold nanoparticles was reported by Frey et al. ${ }^{94}$ PNIPAM was first prepared via free radical polymerization in aqueous medium. Next, gold-PNIPAM colloids were produced from aqueous mixtures of $\mathrm{HAuCl}_{4}$ and PNIPAM using ascorbic acid as reducing agent at ambient temperature to yield gold nanoparticles, onto which PNIPAM was adsorbed by weak interactive forces.

Wie et al. ${ }^{97}$ immobilized a disulfide initiator on the surface of gold nanorods and grew PNIPAM brushes using CUBr/PMDETA as catalyst in a mixed solvent system of $\mathrm{H}_{2} \mathrm{O} / 2$ propanol/DMF. To show the feasibility of this system for drug delivery, norvancomycin hydrochloride (NVan, a hydrophilic drug) was loaded into these core-shell gold-PNIPAM particles. They anticipated that at $25{ }^{\circ} \mathrm{C}$ (below the LCST) drug molecules adsorbed to PNIPAM through hydrogen bonding. It was demonstrated that the rate of drug release was faster during laser exposure due to collapse of PNIPAM chain as a result of gold nanorod heating from 25 to $41^{\circ} \mathrm{C} .{ }^{97}$ Clinical translation of this system is limited due to the fact that PNIPAM at $37^{\circ} \mathrm{C}$ is already hydrophobic and will thus show untriggered fast drug release at body temperature.

Wang et al..$^{98}$ prepared positively charged gold nanorods (GNR) ${ }^{99}$ and coated them with negatively charged $\mathrm{p}$ (NIPAM-MAA) at $\mathrm{pH} 7.4$ to obtain a core-shell nanosphere system. The GNRs in the core of nanosphere were able to adsorb and convert light to heat upon irradiation of the nanospheres with a near-infrared (NIR) laser. The nanospheres with an average size of $110 \mathrm{~nm}$ exhibited an LCST of $40{ }^{\circ} \mathrm{C}$ at $\mathrm{pH}$ 7.4. The hydrophilic drug 5fluorouracil (5-FU) was loaded to the nanospheres by electrostatic interaction between negatively charged carboxyl moieties of MAA and positively charged amine groups in 5-FU. They observed a cumulative release of $71 \%$ in $12 \mathrm{~h}$ by reducing the $\mathrm{pH}$ to 5.5 , resulting in protonation of carboxylate groups and consequently, shrinking of the particle shell. Also, cumulative release at $\mathrm{pH} 6.6$ increased from $20 \%$ to $45 \%$ in about $3 \mathrm{~h}$ after irradiation of nanospheres with NIR light (for 4 cycles of 60s) due to elevation of temperature and consequently an increase in hydrophobicity of the shell. The effect of nanosphere-loaded 5 -FU on tumor growth inhibition was investigated in vivo in mice. They observed that the 
nanosphere-loaded 5-FU formulation in combination with irradiation resulted in significant inhibition of tumor growth in comparison with free drug and the 5-FU formulation without irradiation. $^{98}$

Magnetite nanoparticles with a size below the superparamagnetic limit show on-off magnetic switching behavior in a magnetic field. ${ }^{100}$ This feature of magnetite nanoparticles has attracted a lot of attention in the biomedical field e.g. for magnetic resonance imaging, (triggered) drug delivery, and biosensors. ${ }^{101,102}$ On demand drug diffusion from nanocomposite membranes consisting of magnetite nanoparticles and PNIPAM based nanogels was demonstrated by Hoare et al. ${ }^{103}$ They synthesized a copolymer of NIPAM, AA, $\mathrm{N}$-isopropylmethacrylamide (NIPMAM), and $N, N^{\prime}$-methylenebisacrylamide by a precipitation polymerization method ${ }^{104}$ to obtain a nanogel with a swelling transition temperature of $43{ }^{\circ} \mathrm{C}$. For membrane preparation, super-paramagnetic magnetite nanoparticles and the nanogels were entrapped in ethyl cellulose as a membrane support by a co-evaporation technique. Conversion of magnetite energy to thermal energy by magnetite nanoparticles resulted in nanogel shrinking and consequently release of a model drug (sodium fluorescein). ${ }^{103}$

\section{Bioconjugation of PNIPAM}

In recent years, there has been a growing interest in biohybrid materials like peptide/protein polymer conjugates due to their potential of combining advantageous properties of both building blocks. ${ }^{105,106,107}$ PEGylation of proteins is a well-known example of bioconjugation, which is used to enhance plasma half-life and reduce immunogenicity of pharmaceutical proteins. ${ }^{108,109}$ Peptide sequences can also be used to introduce interesting properties in polymeric systems. Decoration of polymers with peptides that are substrates for endogenous proteases can be used to trigger and control e.g. hydrogel degradation and drug release in vivo. ${ }^{110}$

The grafting of a stimulus responsive polymer near the active site of a protein can be used to modulate the affinity of the protein for its specific target. Such systems have potential applications for biosensors, affinity separations and immunoassays. These conjugations have also been used for instance to control protein-ligand recognition (Figure 9). ${ }^{111}$ Advanced polymerization techniques like ATRP provide opportunities for the preparation of such hybrid polymer systems. ${ }^{60}$ Amino acid initiators for ATRP have been developed to synthesize compounds with site specific modifications, while retaining control over polymer chain length and composition. ${ }^{112}$

RAFT polymerization is also known as an attractive technique for conjugation of polymers 
to biomaterials. ${ }^{70}$ Coupling of a RAFT agent $(\mathrm{Z}-\mathrm{C}(=\mathrm{S}) \mathrm{S}-\mathrm{R})$ to a protein has been achieved using either its $\mathrm{R}$ or $\mathbf{Z}$ group. The R-group approach provides a hydrolysable thiocarbonylthio moiety at the end of the polymer chains, which can be used in subsequent steps to attach desired functional groups. In addition, a control over molecular weight of the polymer is easier when the CTA residue is not in close proximity to the protein surface due to steric hindrance. ${ }^{113} \mathrm{~A}$ CTA can also be attached to the protein via its Z-group. The advantage of this method is that only dormant 'living' chains are conjugated to the protein, while terminated chains are not. ${ }^{114}$ Moreover, triggered cleavage of the polymer from the protein is possible by e.g. aminolysis due to the relatively labile thiocarbonylthio moiety enabling separate characterization of the polymer. ${ }^{115}$
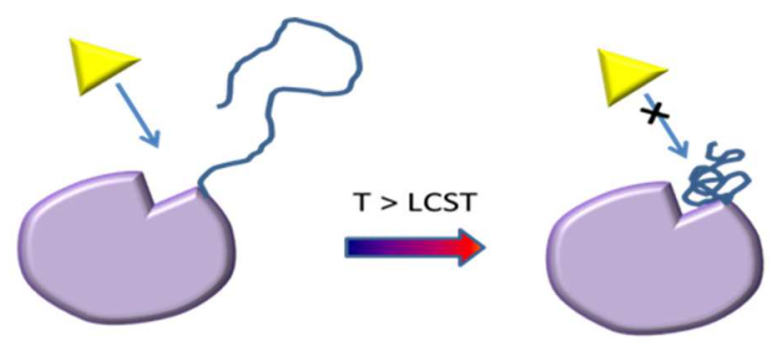

Figure 9: Schematic illustration of conjugation of a stimuli-responsive polymer close to binding pocket of a protein. In the hydrated random coil state, the polymer interferes minimally with ligand binding to the receptor binding pocket. Upon increasing temperature, the collapsed polymer blocks access to the binding pocket.

\subsection{Protein-PNIPAM conjugates}

One of the well-known strategies to synthesize protein-PNIPAM conjugates is the "grafting to" approach in which a functionalized polymer is conjugated to biomaterials. Polymers containing conjugating groups (e.g. NHS) are commonly used for this purpose. For example, Hoffman et al. ${ }^{116}$ conjugated a copolymer of poly(NIPAM-co- $N$-acryloxy succinimide) (P(NIPAM-Co-NAS)) to protein A. Next, the conjugate was used for affinity precipitation separation of human immunogammaglobulin (IgG). Binding of protein A-PNIPAM conjugate to IgG formed a Protein A-PNIPAM/IgG complex. Precipitation of the obtained complex was achieved by increasing the temperature above the LCST, which resulted in easy separation of IgG without the need for chromatographic columns. ${ }^{116}$ Also, conjugation of PNIPAM to proteins through disulfide bonds has been reported. ${ }^{117}$ 
Recently, Lorenzo et al. ${ }^{118}$ used a two ends tetrazine functionalized PNIPAM for homodimerization of T4 lysozyme. This mutant T4 lysozyme (V131C) having a single surfaceexposed cysteine was conjugated to a bis-tetrazine PNIPAM (2 kDa) via tetrazine-trans cyclooctene ligation. As expected, the LCST of the conjugate increased to $39{ }^{\circ} \mathrm{C}$ due to the increased hydrophilicity of the hybrid structure. ${ }^{119}$ They observed that the T4L-PNIPAM-T4L lost $75 \%$ of its activity both above and below the LCST, however, the activity of T4L-PNIPAM was preserved after conjugation. ${ }^{120}$ They speculated that the reduction in its activity, especially towards a large substrate, is due to steric hindrance of the second protein. ${ }^{118}$ Maynard et al. ${ }^{120}$ were the first to develop a PNIPAM-protein conjugate by attaching an ATRP initiator to free thiol residues on proteins. PNIPAM was grown from lysozyme in the presence of $\mathrm{Cu}(\mathrm{I}) \mathrm{Br} / \mathrm{bipy}$ as a catalyst and water as a solvent at $23{ }^{\circ} \mathrm{C}$ ("grafting from" approach). The polymerization process was not detrimental to lysozyme which was demonstrated by comparing its activity with and without PNIPAM both below and above its LCST. ${ }^{120}$

Controlling the activity and stability of an enzyme by grafting stimuli responsive polymers was reported by Russell et al. ${ }^{121}$ They observed an increase in the stability of chymotrypsin after conjugation of PNIPAM to this protein. To synthesize such a bioconjugate, amines present in lysine residues and on the $\mathrm{N}$-terminus of this protein were functionalized with a water-soluble ATRP initiator and PNIPAM chains were subsequently grafted from the protein in aqueous solution in the presence of $\mathrm{CuBr} / \mathrm{Me} 6 \mathrm{TREN}$ as a catalyst at $4{ }^{\circ} \mathrm{C}$. This modification did not only affect the affinity of the chymotrypsinPNIPAM (CT-PNIPAM) for $N$-succinylAla-Ala-Pro-Phe p-nitroanilide as a model substrate, but also influenced the activity and the stability of the

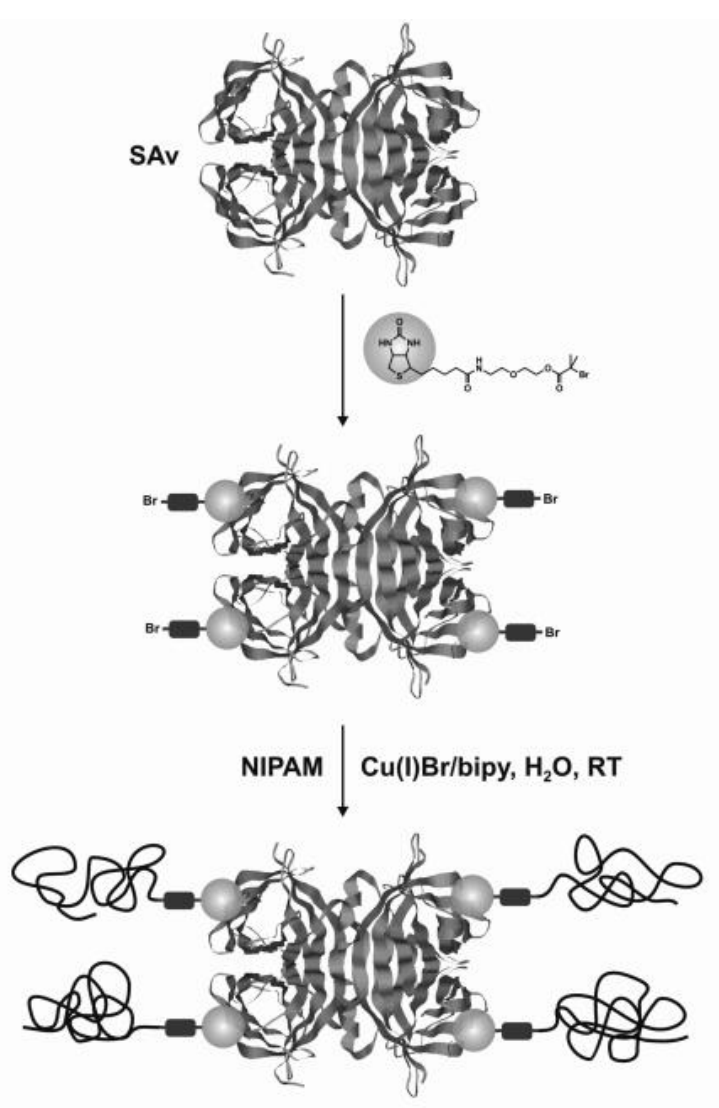

Figure 10: Synthesis of streptavidin-[biotin]4 macroinitiator and streptavidin-[biotin-PNIPAM] bioconjugate. Reported with permission from Ref. ${ }^{60}$ 
enzyme. Not surprisingly, above the LCST of PNIPAM (at $40^{\circ} \mathrm{C}$ ), the affinity for the substrate reduced due to competing hydrophobic interactions of the substrate with PNIPAM chains. ${ }^{121}$

Streptavidin (SAv) modified with four arms of PNIPAM was synthesized by functionalization of the protein with a biotin ATRP initiator. ${ }^{122}$ This polymerization was done at ambient temperature in water using $\mathrm{Cu}(\mathrm{I}) \mathrm{Br} / \mathrm{bipy}$ as a catalyst. At temperatures above the LCST of PNIPAM, precipitation of the polymer occurred, which made it a promising bioconjugate for the development of systems for enzyme assays and bioanalysis (Figure 10). ${ }^{60,122}$

An early example of protein-PNIPAM conjugates obtained by RAFT polymerization was reported by Boyer et al. ${ }^{123}$ The free thiol group of the Cys-34 moiety in bovine serum albumin (BSA) was functionalized with a water-soluble RAFT initiator via its Z-group. They observed polymer-protein aggregation upon increase in the temperature above the LCST of PNIPAM and formation of particles with a size of 250-300 nm. ${ }^{123}$

Also Sumerlin et al. ${ }^{124}$ polymerized NIPAM from BSA but in their publication the RAFT agent was attached via the R-group. A maleimide-functionalized trithiocarbonate was attached to the free thiol group of the Cys-34 residue on BSA at pH 7.2. They reported facile separation of the protein-polymer conjugates from non-conjugated protein just by heating the mixture of conjugate and unmodified BSA above the LCST of PNIPAM $\left(35^{\circ} \mathrm{C}\right) . .^{124}$ To demonstrate the retention of the active thiocarbonylthio moieties on the $\omega$ end of the conjugated chains, the same strategy was used to synthesize BSA-PNIPAM- $b$-PDMA and, as expected, the LCST increased with increasing molecular weight of the hydrophilic PDMA block. ${ }^{125}$ They also block copolymerized NIPAM and DMA from lysozyme by conjugating an $\mathrm{N}$ hydroxysuccinimide (NHS) modified chain transfer agent to amine groups of the protein. To investigate the molecular weight and polydispersity of resulting polymer, the conjugate was treated with Tergazyme to decompose the protein. An average PDI of 1.3 and $M_{n}$ of $31 \mathrm{kDa}$ were obtained after cleavage of the polymer from the protein, which confirmed successful RAFT polymerization of well-defined polymers even in bio-friendly conditions. ${ }^{126}$

\subsection{Peptide-PNIPAM conjugates}

The most commonly applied conjugation methods of polymers to peptides can be categorized by two approaches: ${ }^{127}$ (1) a peptide can be conjugated to a preformed polymer utilizing frequently used coupling methods e.g. click chemistry, ${ }^{128,129}$ or (2) a peptide can act as a macroinitiator and a polymer chain can be grown from the peptide; ${ }^{130}$ For approach 1 , preformed polymers can be used, but purification and functionalization of polymer chains before attachment to a peptide is necessary. For example, Bulmus et al. ${ }^{131}$ copolymerized 
NIPAM and acrylic acid (AA) to obtain P(NIPAM-co-AA) consisting of $30 \%$ acrylic acid with an LCST of $60^{\circ} \mathrm{C}$ at $\mathrm{pH} 7.4$. The pending carboxylic groups of this polymer were functionalized with methyl ester protected alanine and the extent of derivatization was $93 \%$. They observed that conjugation of these alanine methyl esters to the polymer reduced the transition temperature to $41{ }^{\circ} \mathrm{C}$ due to a reduction in hydrophilicity of the polymeric chains by modification of the acid groups. ${ }^{131}$

Conjugation of an immunogenic VNTR peptide present in the cancer associated protein MUC1 to PNIPAM was investigated by Kakwere et al. ${ }^{132}$ RAFT polymerization of NIPAM using an alkyne-functionalized-CTA resulted in polymer chains with an alkyne moiety on one chain end and a thiocarbonylthio group on the other end. After polymerization, an azide containing hydrophilic peptide was clicked to the polymer and subsequent treatment of thiocarbonylthio groups with TCEP resulted in a thiol-functionalized polymer-peptide, which was then coupled to a fluorescent label. Self-assembly of the polymers above the LCST resulted in nanoparticles with the hydrophilic peptide exposed on the surface. The authors suggested that these nanostructures can be used to develop vaccines for cancer therapy. ${ }^{132}$

For approach 2, both RAFT and ATRP have been used for the polymerization of a variety of monomers using a peptide as a macroinitiator. For instance, PNIPAM was grafted to the surface of a peptide nanotube by Couet et al. ${ }^{133}$ To this end, a bromide functionalized cyclic peptide self-assembled into a peptide nanotube. Initiation of living radical polymerization from the surface of this nanotube gave peptide-polymer hybrid nanotubes. A similar system was designed using RAFT polymerization of NIPAM by Daniel et al. ${ }^{134}$ and they confirmed that the obtained constructs were capable of forming artificial channels in a phospholipid bilayer (Figure 11). ${ }^{134}$ They demonstrated that due to the hydrophobic nature of

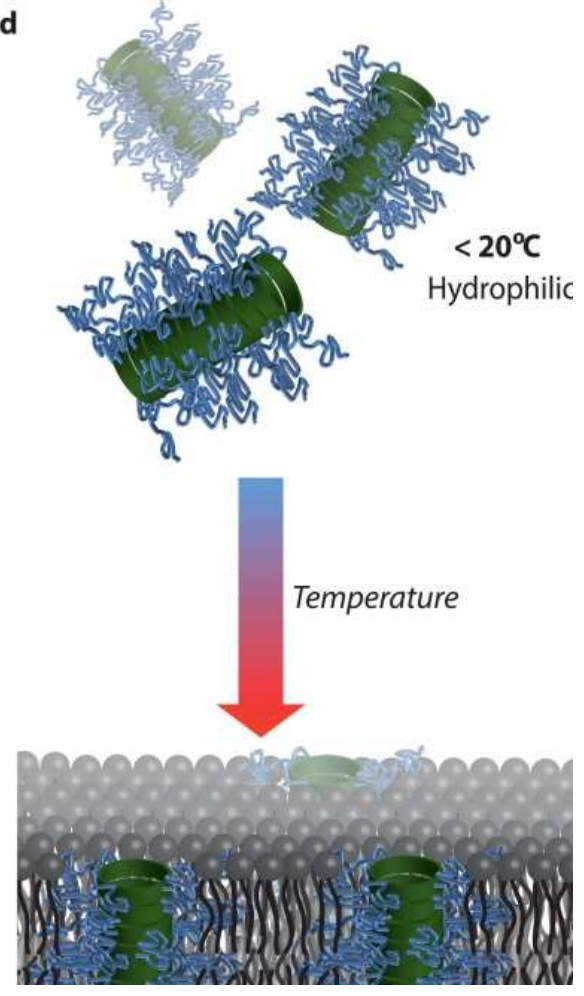

Figure 11: Illustration of well-defined unimeric channels formed upon heating a solution of cyclic peptide-PNIPAM up to an intermediate temperature of $35^{\circ} \mathrm{C}$ in the presence of large unilamellar vesicles (LUVs). Reported with permission from Ref. ${ }^{134}$ 
PNIPAM above the LCST, these nanotubes penetrate into the lipophilic bilayer, which link the cytosol and extracellular media. ${ }^{134}$ Although clinical translation seems rather limited with a transition at $28^{\circ} \mathrm{C}$, technically it is an interesting concept.

Trzebicka et al. ${ }^{135}$ conjugated a cleavable synthetic peptide to PNIPAM. A pentapeptide initiator was grafted to the surface of a polystyrene resin and used as ATRP macroinitiator. Polymerization of PNIPAM was performed using $\mathrm{CuCl} / \mathrm{Me}_{6}$ TREN as a catalyst in a mixed solvent of DMF/water, and subsequently the polymer-peptide conjugate was cleaved from the resin. The hydrophilic nature of the peptide caused a higher transition temperature (34 ${ }^{\circ} \mathrm{C}$ ) for the PNIPAM-peptide conjugate than that of PNIPAM. Enzymatic degradation of the peptide sequence was possible at temperatures both above and below the LCST indicating that the peptide residues coupled to PNIPAM are accessible for a matching enzyme. ${ }^{135}$ De Graaf et al. ${ }^{136}$ used the thermosensitivity of PNIPAM for designing an enzyme-triggered drug delivery system. They positioned a cleavable peptide between a thermosensitive (PNIPAM) and hydrophilic poly(oligo (ethylene glycol) methyl ether methacrylate) (POEGMA) block using ATRP. For this purpose, a cleavable peptide sequence was functionalized at its C-terminus with an ATRP initiator to polymerize OEGMA. This step was followed by functionalization of the $N$-terminus of peptide-POEGMA with another ATRP initiator to perform polymerization of NIPAM. Inactivation of the end chain after polymerization of the first block, by substitution of its $\mathrm{Cl}$-terminus with an azide group provided an extra opportunity for attaching a functional group such as a fluorescent label by click chemistry. Furthermore, the hydrophilic corona of micelles formed by these amphiphilic polymers could be cleaved by metalloproteinases (MMPs), ${ }^{136}$ which are upregulated in inflamed tissues. ${ }^{137}$

Borner et al. ${ }^{138}$ reported the coupling of a RAFT agent via the R end to a GGRGDS peptide. Subsequently, they used this initiator for RAFT polymerization of NIPAM, and after hydrolysis of the resulting polymer, a thermosensitive GGRGDS-PNIPAM-SH conjugate was obtained. These conjugates were subsequently grafted onto planar gold surfaces and used in a cell adhesion study. Their results illustrated that above the LCST of the polymer, the cells could be attached to the surface as the hydrophobic behavior of the polymer was dominant. Lowering temperature below the LCST caused cell detachment within 30 min because of hydrophilization of the surface. Also, they observed that cell adhesion to the surface was improved by the presence of the peptide sequence. ${ }^{138}$

\subsection{Nucleic acid-PNIPAM conjugates}


Conjugation of single or double stranded DNA fragments to a polymer aimed for applications in the fields of biosensors, imaging, and drug delivery has been studied extensively. ${ }^{139,140141}$ Maeda et al. published a number of papers dealing with DNA-PNIPAM conjugates and their application for affinity precipitation/separation of DNA-binding molecules. ${ }^{142,143,144}$ For instance, they reported sequence-specific precipitation separation of oligonucleotides. To this end, free radical copolymerization of NIPAM with vinylderivatized oligonucleotide monomers was used to obtain an SsDNA-PNIPAM conjugate. This conjugate was able to interact with complementary oligonucleotide sequences in solution and subsequent separation could be achieved by increasing the temperature above the LCST. ${ }^{145,146}$

Maeda et al. ${ }^{147}$ also reported an example of the synthesis of DNA-diblock copolymers by RAFT polymerization using 4-cyanopentanoic acid dithiobenzoate as CTA. After polymerization of NIPAM in DMF at $60^{\circ} \mathrm{C}$, the dithiobenzoate end group was hydrolyzed to obtain PNIPAM-SH. The resulting polymer was subsequently covalently linked to a maleimide-modified single DNA strand to yield a PNIPAM-DNA diblock copolymer. Above the LCST, the polymeric chains self-assembled into micelles with a PNIPAM hydrophobic core and a hydrophilic DNA corona. The micelles had excellent stability even in high ionic strength medium due to electrostatic and entropic repulsion between ssDNA on the shell of micelles. The colloidal stability of the micellar dispersion was reduced significantly after addition of complementary DNA strands and formation of fully matched dsDNA. Less flexibility of dsDNA in comparison with ssDNA resulted in reduced entropic repulsion, ${ }^{148}$ and consequently aggregation of the micelles. ${ }^{147}$ This system has potential application in affinity separation of DNA-binding molecules. However, the application for gene delivery is expected to be limited due to the presence of DNA in the shell of micelles and its accessibility to nucleases present in extracellular fluids.

\section{Liposome surface modification with PNIPAM}

Liposomes are nanosized systems consisting of an aqueous core surrounded by one or more lipid membranes, which have been developed among others for the encapsulation of chemotherapeutic drugs to improve the therapeutic efficacy and to reduce adverse events. ${ }^{149,150}$ Drug release from liposomes can be triggered by external factors such as changes in $\mathrm{pH}$, light and ultrasound exposure ${ }^{151,152}$ but so far heat has been the most intensively studied trigger for drug release. Two main groups of temperature sensitive liposomes have been developed: liposomes containing thermosensitive lipids e.g. 1,2dipalmitoyl-sn-glycero-3-phosphocholine $\quad$ (DPPC), 1,2-distearoyl-sn-glycero-3- 
phosphocholine (DSPC); ${ }^{153}$ and liposomes that are decorated with thermosensitive polymers e.g. PNIPAM. ${ }^{154,155}$

The mechanism of drug release from thermosensitive liposomes is related to the melting phase transition temperature $\left(T_{m}\right)$ of the phospholipids. At this temperature, the lipids undergo a phase transition from a solid gel phase to a liquid crystalline phase, which causes phase separation of the lipid bilayer that is associated with its permeabilization. ${ }^{156,157}$ In case of polymer coated liposomes, the thermosensitive polymer undergoes a coil-to-globe transition upon passing the LCST, which can induce destabilization of liposomes in a controlled manner.

Temperature triggered drug release from PNIPAM coated liposomes was pioneered by Kono et al. ${ }^{152,154,158}$ (Figure 12). Another example of modification of liposomes by thermosensitive polymers was reported by Dong Han et al. ${ }^{159}$ They modified liposomal surfaces with $\mathrm{P}($ NIPAM-AAM) and polyethyleneglycol (PEG) to tune the release profile of encapsulated doxorubicin (DOX) and enhance the stability of these liposomes in blood. An increase in DOX release was observed in a temperature range close to the LCST of the polymer due to a coilglobule transition of the polymer that above the LCST interacts with the bilayer resulting in destabilization of the liposomal membranes. ${ }^{159}$

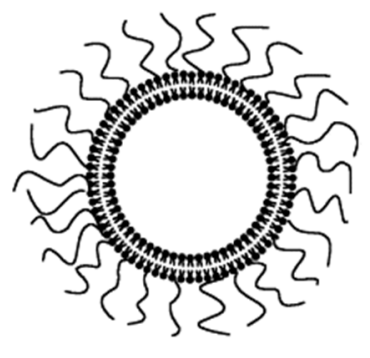

T<LCST

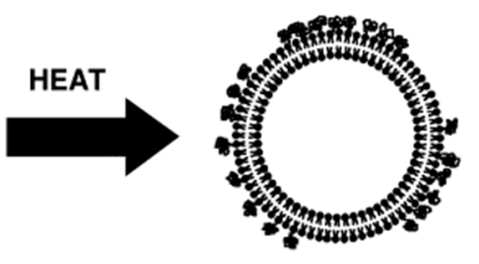

LCST $<\mathrm{T}$

Figure 12: Schematic illustration of design of temperature-sensitive liposomes using a thermosensitive polymer. The liposome is stabilized by hydrated polymer chains below its LCST. However, above the LCST, the dehydrated and contracted polymer chains cause destabilization of the liposome, an increase in hydrophobicity of the liposome surface, and/or exposure of the bare liposome surface. Reported with permission from Ref. 155

\section{Applications of PNIPAM in cell culture}

The temperature sensitive behaviour of PNIPAM has been exploited by Okano et al. ${ }^{160,161,162}$ 
for controling cell attachment and detachment from polymeric substrates. Efficient recovery of cells from the polymeric substrate is a crucial step for successful cell passage and characterization. Cells are able to attach, spread and proliferate on relatively hydrophobic PNIPAM-modified surfaces above the LCST. By lowering the temperature below the LCST, cell adhesion to the surface will be weakened as result of hydration of the NIPAM coated surface (Figure 13). For example, Schmaljohann et al. ${ }^{163}$ immobilized a diblock copolymer of PEGPNIPAM onto tissue culture substrates by low-pressure argon plasma treatment. ${ }^{164}$ They observed that by lowering the temperature to $34{ }^{\circ} \mathrm{C}$ (below the LCST), cells detached from the surface in 20 min due to the increase in surface hydrophilicity. ${ }^{163}$

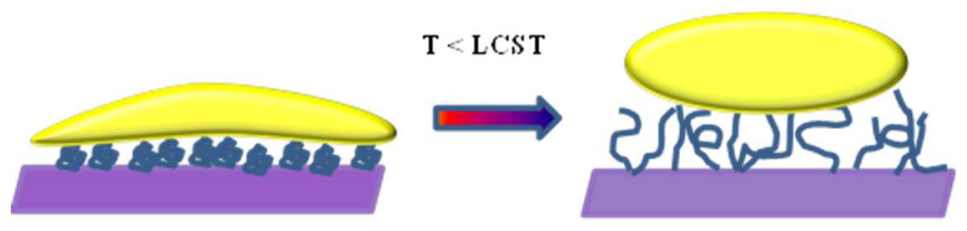

Figure 13. Schematic representation of the adhesion above the LCST and detachment below the LCST of a cell on a PNIPAM-grafted surface.

\section{Crosslinking methods for polymers}

There has been considerable attention for crosslinking methods of polymeric structures to modulate their properties for aimed applications. For instance, in hydrogels, the degree of crosslinking is an important factor used to tune their mechanical and physical properties, modulate swelling and shrinking behavior, and thereby gaining control over porosity of the hydrogel. ${ }^{165}$ Crosslinking also improves micelle stability and prevents their dissociation even at low concentrations. ${ }^{166,167}$

The selected method of crosslinking (chemical or physical) strongly affects the properties of polymeric materials. Among different chemical crosslinking methods, we can distinguish between crosslinking by radical polymerization or chemical reaction of complementary groups. Physical crosslinking methods make use of non-covalent interactions such as ionic interactions, hydrogen bonding, hydrophobic interactions, and crystal formation. ${ }^{168}$ Thermoresponsive self-assembling behavior of PNIPAM is also an example of physical crosslinking (see section 2 ).

\subsection{Crosslinking in PNIPAM based hydrogels}

Hydrogels are materials used for controlled drug delivery, as cell culture matrices and for 
tissue engineering applications. ${ }^{169}$ Due to their high water content and soft nature, hydrogels mimic the physicochemical properties of native extracellular matrices and therefore have, generally speaking, a good biocompatibility. Facile mobility of water, proteins, and nutrients within hydrogel matrices along with their mechanical support and softness make them attractive materials for pharmaceutical and biomedical applications. ${ }^{170,171}$ Several studies have been published on thermosensitive hydrogels based on PNIPAM as reviewed in detail by Park et al. ${ }^{172}$ and Klouda. ${ }^{173}$

Biodegradability of hydrogels should also be taken into account for biomedical and pharmaceutical applications and therefore hydrogels are often designed bearing labile bonds either in the polymer backbone or in the crosslinks. For example, disulfide bonds and ester bonds can be degraded by reduction or hydrolysis, respectively. Hydrolysis is the most studied mechanism for hydrogel degradation but there is an increasing interest in reductive and enzymatic processes to induce degradation of mainly nano sized hydrogels for e.g. intracellular drug delivery. ${ }^{174,175}$

A PNIPAM based hydrogel exhibits a sharp reversible swelling-deswelling transition below and above the LCST, which is also referred to as the volume phase transition temperature (VPTT) usually around $32-34{ }^{\circ} \mathrm{C}$ in aqueous media. ${ }^{176,177}$ Hydrophobicity and hydrophilicity of PNIPAM based hydrogels can be tuned by copolymerization with different monomers, which in turn affect the volume phase transition temperature. In general, the hydrogel response to stimuli is strongly affected by the crosslink density. Increasing the crosslink density decreases the swelling of PNIPAM based gels while only slightly affecting the critical volume-transition temperature. ${ }^{178}$ Furthermore, crosslink density impacts stiffness of the gel, which should be considered when designing e.g. scaffolds for tissue engineering.

Amongst the different methods of crosslinking, in situ crosslinking of hydrogels is relatively novel and attractive for many applications. In situ crosslinking allows the design of injectable hydrogels that can be used for minimally invasive treatments and to potentially fill irregularly shaped defect sites. However, toxicity of byproducts or catalysts of crosslinking reactions should be considered carefully ${ }^{179}$. Boere et al. ${ }^{180}$ developed an injectable PNIPAM hydrogel with a dual hardening mechanism. An ABA tri-block copolymer of PNIPAM, $\mathrm{N}$-(2hydroxypropyl)methacrylamide-cysteine (HPMA-Cys) as thermosensitive block and PEG as hydrophilic block (P(NIPAM-co-(HPMA-Cys)-PEG-(NIPAM-co-(HPMA-Cys)) was synthesized by free radical polymerization. PEG-thioester functionalized linkers were used to crosslink the hydrophobic domains after thermogelation via native chemical ligation (NCL). ${ }^{180} \mathrm{~A}$ major advantage of native chemical ligation over other chemical crosslinking methods is that this ligation proceeds without the need for a catalyst, at ambient temperature and in aqueous media at physiological $\mathrm{pH}$. This reaction involves coupling of an $\mathrm{N}$-terminal cysteine to a 
thioester resulting in a thioester-linked intermediate product that after spontaneous S-Nacyl rearrangement yields an amide bond (Figure 14). The ligation is highly specific and hence only a single product is expected even in the presence of additional cysteine residues present in e.g. proteins. In a follow-up study, NHS-functionalized PEG was used for crosslinking and dimethyl- $\gamma$-butyrolactone acrylate (DBA) was introduced as a hydrolysable monomer in the thermosensitive blocks. In this case, the same reaction results in the release of $\mathrm{N}$-hydroxysuccinimide after oxo-ester native chemical ligation in contrast to the previously system that released a thioester byproduct. The biodegradability of the hydrogel was tunable by the DBA content and varied between a few days up to several months showing the versatility of this system as an injectable hydrogel. It was also demonstrated that the crosslinking density of the hydrogel network could be controlled by the amount of cysteine groups present in the thermosensitive block and using 8-arm functionalized PEG instead of linear functionalized PEG. ${ }^{181}$

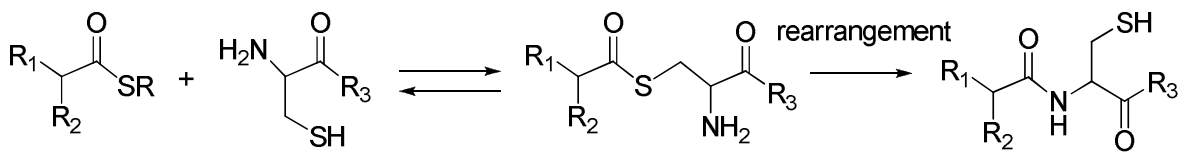

Figure 14: Native chemical ligation of thioester and $\mathrm{N}$-terminal cysteine.

Non-degradability issues of PNIPAM can also be tackled by grafting PNIPAM to a degradable polymer. For this purpose, Marra et al. ${ }^{182}$ developed an injectable, degradable PNIPAM gel by grafting PNIPAM to aminated hyaluronic acid. These AHA-g-PNIPAM copolymers exhibited an LCST of around $30^{\circ} \mathrm{C}$. It was observed that the enzymatic degradability of the hydrogel was highly affected by the weight ratios of PNIPAM. Hydrogels with higher PNIPAM grafting degree exhibited a lower rate of degradation. ${ }^{182}$

A two component degradable, injectable hydrogel loaded with magnetite nanoparticles was first described by Hoare et al. ${ }^{183}$ Free radical copolymerization of NIPAM and acrylic acid resulted in P(NIPAM-s-AA). After functionalization of carboxylic acid groups of the polymer with hydrazide groups, the polymer was peptized to the surface of magnetite nanoparticles. This hydrazide PNIPAM-magnetite conjugate was mixed with aldehyde functionalized dextran to crosslink the polymer-magnetite particles via hydrolysable hydrazone bonds. The resulting composite had a high elasticity $\left(G^{\prime}>60 \mathrm{kPa}\right)$ even at high water content $(>82 \%)$. Bupivacaine hydrochloride was loaded into this composite material to investigate possible application as a pulsatile drug delivery system. A significant increase in the rate of drug release after exposure of the gel to a magnetic field was indeed observed (Figure 15), ${ }^{183}$ showing that this hydrogel is an attractive example of a dual sensitive material. 


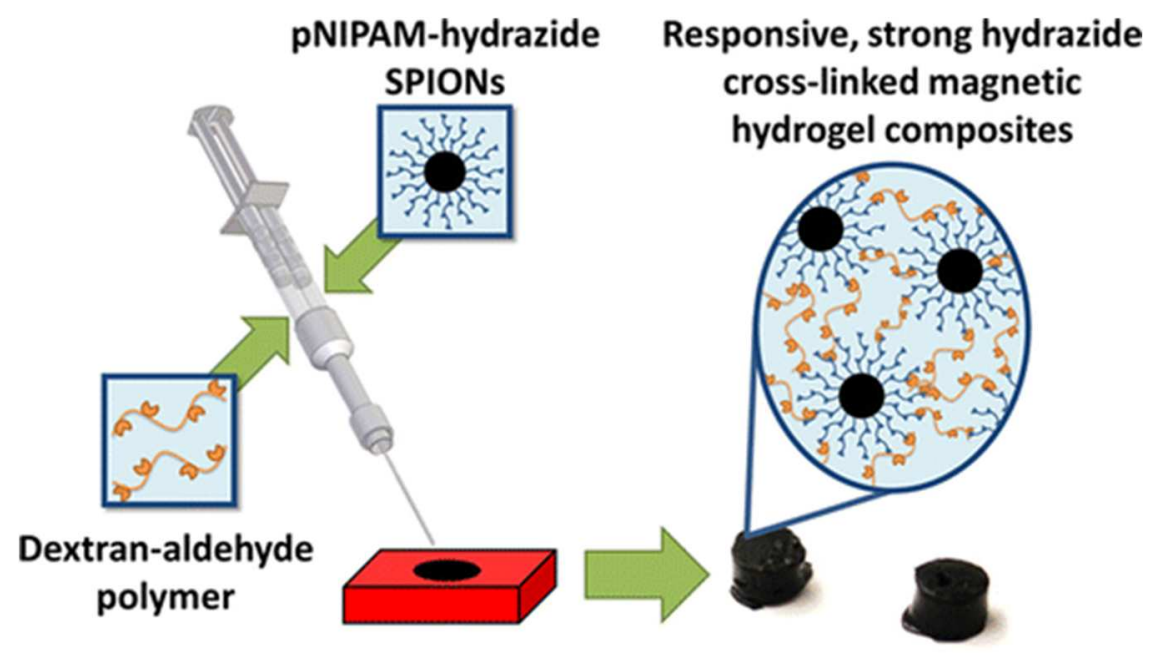

Figure 15: Hydrogel fabrication process. A double barrel syringe in which one barrel contains the PNIPAM-hydrazide-coated SPIONs and the others contain the dextran-aldehyde hydrogel precursor was used to prepare the composite disks for testing. Both materials are dissolved/suspended in PBS, with a pharmaceutical agent of interest dissolved in both barrels if desired. Upon injection, the solutions are intimately mixed in a static mixing channel before being injected into a silicone mold through a needle tip for the formation of the test composite magnetic disks. Reproduced with permission from Ref. ${ }^{183}$

Crosslinking of a PNIPAM based polymer via click reaction was reported by Zhang et al. ${ }^{184}$ RAFT polymerization of NIPAM and propargyl acrylate resulted in a polymer with pendant alkyne groups. An azide functionalized cystamine was used to crosslink this polymer through copper catalyzed click chemistry. The obtained hydrogel showed quick and reproducible swelling-shrinkage behavior upon temperature switching between 20 and $40{ }^{\circ} \mathrm{C}$. The presence of disulfide bonds in the crosslinks ensures degradability of the hydrogel in a reductive environment. ${ }^{184}$

A degradable PNIPAM hydrogel with ester linkages both in the polymer backbone and in the crosslinker was reported by Galperin et al. ${ }^{185}$ In this study, NIPAM was photocopolymerized with 2-methylene-1,3-dioxepane (MDO) and polycaprolactone dimethacrylate (PCLDMA). They observed that upon increase in the temperature to $37^{\circ} \mathrm{C}$, the hydrogel swelling decreased. Cell culture studies conducted with this hydrogel illustrated that not only the hydrogel but also the released degradation products were non-toxic. ${ }^{185}$

Vernon et al. ${ }^{186}$ fabricated a PNIPAM gel using Michael-type reactions for crosslinking. They copolymerized NIPAM with $\mathrm{N}$-acryloxysuccinimide (NAS) by free radical polymerization. The NHS groups were then substituted with cysteamine to obtain free thiol groups in the 
polymer chains after a one step reduction. Subsequent Michael-type reaction of free thiol groups with poly(ethylene glycol) diacrylate resulted in a PNIPAM based hydrogel. Not surprisingly, the gel swelling was highly dependent on temperature and crosslinking density. ${ }^{186}$

\subsection{Crosslinking of PNIPAM based micelles}

The importance of crosslinking of micelles has been acknowledged for their application in drug delivery. ${ }^{187,188,189}$ It is known that micelles spontaneously dissociate upon dilution below their critical micelle concentration (CMC). ${ }^{190}$ This dissociation can lead to premature release of a loaded drug from the micelles upon administration e.g. in the circulation. ${ }^{191}$ It has been shown that the stability of micelles can be enhanced considerably by crosslinking the polymer chains in the core or shell, which are classified as either core cross-linked $(C C L)^{192}$ or shell cross-linked (SCL) ${ }^{167,193}$ micelles.

\subsubsection{Shell cross-linked (SCL)}

An example of shell crosslinking of PNIPAM based micelles was reported by McCormick et al. ${ }^{194}$ who used RAFT polymerization to synthesize a PEO- $b$-P(DMA-s-NAS)- $b$-PNIPAM triblock copolymer. This copolymer dissolved in water to form micelles above the LCST of the PNIPAM block and it was shown that bifunctional as well as multifunctional primary aminecontaining compounds can be used for crosslinking of the DMA-s-NAS block. ${ }^{194}$ In a follow up paper, they introduced crosslinking of the same polymer blocks with cystamine. Micellization of this polymer in aqueous solution occurred at $37{ }^{\circ} \mathrm{C}$ and subsequent crosslinking of the mid-block of the micelles with cystamine resulted in SCL micelles with a PNIPAM core. Dipyridamole (DIP), as a model drug, was loaded into the micelles and it was shown that in the presence of reducing agents such as DTT and TCEP cleavage of the crosslinks occurred resulting in release of the loaded drug. ${ }^{195}$

Shell crosslinking of polymeric micelles by the reaction of aldehydes with amines was investigated by Li et al. ${ }^{196} \mathrm{~A}$ Y-shaped polymer of PNIPAM- $b$-(poly(L-lysine) (PLL)) 2 was synthesized by a combination of ATRP, ROP and click chemistry. This polymer dissolved in water and self-assembled into micelles with a PNIPAM core and a PLL shell. Addition of glutaraldehyde to the micelles yielded $S C L$ as a result of a reaction between primary amines and aldehydes. These micelles were loaded with the hydrophobic drug prednisone acetate and, as expected, the release of the drug was dependent on the crosslink density and temperature. ${ }^{196}$ However, the application of glutaraldehyde crosslinked biomaterials can be 
considered as controversial due to reported cytotoxicity of this compound. ${ }^{197}$ Crosslinking of micelles can also be performed by organic-inorganic shell-crosslinking methods. ${ }^{198}$ An example of such a hybrid system was reported by Wei et al. ${ }^{199}$ who synthesized a block copolymer of NIPAM and 3-(trimethoxysilyl) propyl methacrylate (MPMA) as hydrophilic block A and poly(methyl methacrylate) as hydrophobic block B. Silica crosslinking of micelles was triggered under acidic conditions after micellization. An in vitro release study of a model drug (prednisone acetate) showed that the crosslinked micelles not only displayed higher loading but also slower drug release in comparison with the noncrosslinked micelles. ${ }^{199}$

\subsubsection{Core cross-linked (CCL)}

Redox-sensitive core crosslinked PNIPAM based micelles were reported by Narain et al. ${ }^{200}$ They copolymerized NIPAM and bis(2-methacryloyloxyethyl) disulfide (DSDMA) using a poly(2-aminoethylmethacrylamide) (PAEMA) macro-RAFT agent and 4,4'-azobis(4cyanovaleric acid) (ACVA) as initiator at $70{ }^{\circ} \mathrm{C}$, in a dioxane/water mixture. This polymerization proceeded and immediately $\mathrm{CCL}$ micelles were formed with a PAEMA shell and P(NIPAM-Co-DSDMA) as core. The resulting micelles exhibited reversible swelling behavior within a temperature range of $28-32{ }^{\circ} \mathrm{C}$. Importantly, the disulfide bonds in the cross-linked core imparts redox sensitivity to the micelles and as result incubation with DTT at $40^{\circ} \mathrm{C}$ for 1 day resulted in a clear solution, which showed micelle degradation in reductive conditions. This system has potential application for intracellular drug delivery. ${ }^{200}$

Core crosslinking of micelles based on click chemistry was reported by Zhang et al. ${ }^{201}$ They copolymerized 3-azidopropyl methacrylate (AzPMA) and tert-butyl methacrylate (tBMA) by ATRP. Subsequent click reaction with monoalkyne-terminated PNIPAM resulted in P(tBMAco-AzPMA)-g-PNIPAM. Next, they hydrolyzed $t$-BMA groups to obtain P(MAA-co-AzPMA)- $g$ PNIPAM, as anionic polymer chains. Similarly, ATRP copolymerization of AzPMA and $N, N$ dimethylacrylamide (DMA) and further grafting of monoalkyne-PNIPAM to this construct yielded P(DMA-co-AzPMA)-g-PNIPAM. In the next step, the amine groups of the DMA units of this polymer were fully quaternized with methyl iodide $\left(\mathrm{CH}_{3} \mathrm{l}\right)$ to form a positively charged polymer of P(QDMA-co-AzPMA)-g-PNIPAM. Mixing these two oppositely charged polymers resulted in polyion complex (PIC) micelles with PNIPAM as shell and complexed blocks of P(QDMA-co-AzPMA) and P(MAA-co-AzPMA)-g-PNIPAM as core. Then, the core of the PIC micelles was crosslinked via click chemistry using propargyl ether as an alkyne bifunctional molecule. They observed that the resulting micelles showed a higher stability than noncrosslinked micelles at various $\mathrm{pH}$ and in salt containing solutions at $25^{\circ} \mathrm{C}$. Above $35^{\circ} \mathrm{C}$, 
macroscopic phase separation was observed due to aggregation of the PNIPAM based corona. The polar nature of the polyion complex cores introduces promising features as nano-sized carriers for delivery of charged therapeutic agents. ${ }^{201}$ However, degradability of the polymers for pharmaceutical applications should be considered.

\section{Conclusion and outlook of applications of PNIPAM}

In this chapter, we highlighted examples of PNIPAM polymers of different compositions and architectures and their potential applications in the biomedical and pharmaceutical research fields. NIPAM can be polymerized by a wide range of polymerization methods to produce thermosensitive PNIPAM. The thermosensitive properties around physiological relevant temperatures provide many opportunities to prepare triggerable biomaterials. Combination with other stimuli-responsive molecules, such as $\mathrm{pH}$ - and photo-sensitive functional groups allows even more prospects to engineer environmentally sensitive systems that can be used for the design of smart materials.

The advanced controlled radical polymerization techniques available nowadays enabled scientists to broaden the horizon to well-defined hybrid polymer-protein materials. Conjugation of PNIPAM to peptides and proteins offers possibilities for e.g. bioseparation and biosensing applications. For many biomedical applications, degradability of polymers in physiological conditions is essential and PNIPAM as such does not to fulfil this requirement. Nevertheless, copolymerization of NIPAM with degradable monomers such as DBA and HPMA-lac yield polymers and materials that overcome this issue, keeping the door wide open for PNIPAM based materials in biomedicine. Importantly, outside the body, the thermoreversibility of PNIPAM at $32{ }^{\circ} \mathrm{C}$ has shown to yield unsurpassed properties for in vitro applications, illustrated by the group of Okano et al. who demonstrated that PNIPAM functionalized substrates are excellent coatings for the preparation of cell sheets.

In addition, the extensive research done on PNIPAM and its copolymers has generated inspiration to design other thermosensitive polymers and fully biodegradable materials that potentially have applications for drug delivery and tissue engineering. Furthermore, the research done on PNIPAM resulted in better fundamental insights into the physical properties of temperature sensitive polymers and materials.

\section{Acknowledgements:}

The Dutch Technology Foundation STW (VIDI grant \#13457) is acknowledged for financial support. 


\section{References:}

(1) Yin, X.; Hoffman, A. S.; Stayton, P. S. Biomacromolecules 2006, 7, 1381-1385.

(2) Heskins, M.; Guillet, J. E. J. Macromol. Sci. Part A - Chem. 1968, 2, 1441-1455.

(3) Schild, H. G. Prog. Polym. Sci. 1992, 17, 163-249.

(4) Winnik, F. M.; Ringsdorf, H.; Venzmer, J. Macromolecules 1990, 23, 2415-2416.

(5) Wang, Y. P.; Yuan, K.; Li, Q. L.; Wang, L. P.; Gu, S. J.; Pei, X. W. Mater. Lett. 2005, 59, 17361740.

(6) Kobayashi, M.; Matsumoto, Y.; Uchiyama, M.; Ohwada, T. Macromolecules 2004, 37, 4339-4341.

(7) Senel, S.; Isik-Yuruksoy, B.; Cicek, H.; Tuncel, A. J. Appl. Polym. Sci. 1997, 64, 1775-1784.

(8) Ganachaud, F.; Monteiro, M. J.; Gilbert, R. G.; Dourges, M. A.; Thang, S. H.; Rizzardo, E. Macromolecules 2000, 33, 6738-6745.

(9) López, G. P. Langmuir 2003, 19, 2545-2549.

(10) Magerl, D.; Philipp, M.; Qiu, X.-P.; Winnik, F. M.; Müller-Buschbaum, P. Macromolecules 2015, 48, 3104-3111.

(11) Rao, G. V. R.; Krug, M. E.; Balamurugan, S.; Xu, H.; Xu, Q.; Lo, G. P. Chem. Mater. 2002, 14, 5075-5080.

(12) Hu, J.; Liu, S. Macromolecules 2010, 43, 8315-8330.

(13) Liu, R.; Fraylich, M.; Saunders, B. R. Colloid Polym. Sci. 2009, 287, 627-643.

(14) Halperin, A.; Kröger, M.; Winnik, F. M. Angew. Chemie Int. Ed. 2015, 54, 15342-15367.

(15) Bischofberger, I.; Calzolari, D. C. E.; De Los Rios, P.; Jelezarov, I.; Trappe, V. Sci. Rep. 2014, $4,4377$.

(16) Torii, H.; Tatsumi, T.; Tasumit, M. J. Raman Spectrosc. 1998, 29, 537-546.

(17) Cheng, H.; Shen, L.; Wu, C. Macromolecules 2006, 39, 2325-2329.

(18) Liu, M. S.; Taylor, C.; Chong, B.; Liu, L.; Bilic, A.; Terefe, N. S.; Stockmann, R.; Thang, S. H.; Silva, K. De. Eur. Polym. J. 2014, 55, 153-159.

(19) Lai, H.; Wu, P. Polymer 2010, 51, 1404-1412.

(20) Djokpé, E.; Vogt, W. Macromol. Chem. Phys. 2001, 202, 750-757.

(21) Hore, M. J. A.; Hammouda, B.; Li, Y.; Cheng, H. Macromolecules 2013, 46, 7894-7901.

(22) Costa, R. O. R.; Freitas, R. F. S. Polymer 2002, 43, 5879-5885.

(23) Zhou, X.; Li, J.; Wu, C.; Zheng, B. Macromol. Rapid Commun. 2008, 29, 1363-1367.

(24) Boutris, C.; Chatzi, E. G.; Kiparissides, C. Polymer 1997, 38, 2567-2570.

(25) Furyk, S.; Zhang, Y.; Ortiz-Acosta, D.; Cremer, P. S.; Bergbreiter, D. E. J. Polym. Sci. Part A Polym. Chem. 2006, 44, 1492-1501.

(26) Van Durme, K.; Van Assche, G.; Van Mele, B. Macromolecules 2004, 37, 9596-9605. 
(27) Afroze, F.; Nies, E.; Berghmans, H. J. Mol. Struct. 2000, 554, 55-68.

(28) Tong, Z.; Zeng, F.; Zheng, X.; Sato, T. Macromolecules 1999, 32, 4488-4490.

(29) Schild, H. G.; Tirrell, D. A. J. Phys. Chem. 1990, 94, 4352-4356.

(30) Baltes, T.; Garret-Flaudy, F.; Freitag, R. J. Polym. Sci. Part A Polym. Chem. 1999, 37, 2977-2989.

(31) Tiktopulo, E. I.; Uversky, V. N.; Lushchik, V. B.; Klenin, S. I.; Bychkova, V. E.; Ptitsyn, O. B. Macromolecules 1995, 28, 7519-7524.

(32) Takei, Y. G.; Aoki, T.; Sanui, K.; Ogata, N.; Okano, T.; Sakurai, Y. Bioconjug. Chem. 1993, 4, 42-46.

(33) Costa, M. C. M.; Silva, S. M. C.; Antunes, F. E. J. Mol. Liq. 2015, 210, 113-118.

(34) Loh, W.; Teixeira, L. A. C.; Lee, L. J. Phys. Chem. B 2004, 108, 3196-3201.

(35) Zhang, Y.; Cremer, P. Curr. Opin. Chem. Biol. 2006, 10, 658-663.

(36) Zhang, Y.; Furyk, S.; Bergbreiter, D. E.; Cremer, P. S. J. Am. Chem. Soc. 2005, 127, 1450514510.

(37) Odian, G. In Principles of Polymerization; John Wiley \& Sons, Inc.: Hoboken, NJ, USA, 2004; Vol. 58, pp 198-349.

(38) England, R. M.; Rimmer, S. Chem. Commun. 2010, 46, 5767-5769.

(39) Mendes, P. M. Chem. Soc. Rev. 2008, 37, 2512-2529.

(40) Neradovic, D.; van Steenbergen, M. J.; Vansteelant, L.; Meijer, Y. J.; van Nostrum, C. F.; Hennink, W. E. Macromolecules 2003, 36, 7491-7498.

(41) Cui, Z.; Lee, B. H.; Vernon, B. L. Biomacromolecules 2007, 8, 1280-1286.

(42) Seymour, L. W.; Duncan, R.; Strohalm, J.; Kopecek, J. J. Biomed. Mater. Res. 1987, 21, 1341-1358.

(43) Shah, S. S.; Wertheim, J.; Wang, C. T.; Pitt, C. G. J. Control. Release 1997, 45, 95-101.

(44) Neradovic, D.; Hinrichs, W. L. J.; Kettenes-Van Den Bosch, J. J.; Hennink, W. E. Macromol. Rapid Commun 1999, 20, 577-581.

(45) Neradovic, D.; Soga, O.; Van Nostrum, C. F.; Hennink, W. E. Biomaterials 2004, 25, 2409-2418.

(46) Zhang, J.; Cui, Z.; Field, R.; Moloney, M. G.; Rimmer, S.; Ye, H. Eur. Polym. J. 2015, 67, 346-364.

(47) Huber, D. L.; Manginell, R. P.; Samara, M. A.; Kim, B.-I.; Bunker, B. C. Science 2003, 301, 352-354.

(48) Shi, D.; Ma, D.; Dong, F.; Zong, C.; Liu, L.; Shen, D.; Yuan, W.; Tong, X.; Chen, H.; Wang, J. Biosci. Rep. 2010, 30, 149-158.

(49) Cunliffe, D.; De Las Heras Alarcón, C.; Peters, V.; Smith, J. R.; Alexander, C. Langmuir 2003, 19, 2888-2899. 
(50) Topp, M. D. C.; Dijkstra, P. J.; Talsma, H.; Feijen, J. Macromolecules 1997, 30, 85188520.

(51) Handbook of Radical Polymerization; Matyjaszewski, K., Davis, T. P., Eds.; John Wiley \& Sons, Inc.: Hoboken, NJ, USA, 2002.

(52) Nicolas, J.; Guillaneuf, Y.; Lefay, C.; Bertin, D.; Gigmes, D.; Charleux, B. Prog. Polym. Sci. 2013, 38, 63-235.

(53) Schulte, T.; Siegenthaler, K. O.; Luftmann, H.; Letzel, M.; Studer, A. Macromolecules 2005, 38, 6833-6840.

(54) David, G.; Boyer, C.; Tonnar, J.; Ameduri, B.; Lacroix-Desmazes, P.; Boutevin, B. Chem. Rev. 2006, 106, 3936-3962.

(55) Percec, V.; Popov, A. V.; Ramirez-Castillo, E.; Coelho, J. F. J.; Hinojosa-Falcon, L. a. J. Polym. Sci. Part A Polym. Chem. 2004, 42, 6267-6282.

(56) Chiefari, J.; Chong, Y. K. B.; Ercole, F.; Krstina, J.; Jeffery, J.; Le, T. P. T.; Mayadunne, R. T. A.; Meijs, G. F.; Moad, C. L.; Moad, G.; Rizzardo, E.; Thang, S. H.; South, C. Macromolecules 1998, 9297, 5559-5562.

(57) Matyjaszewski, K.; Xia, J. Chem. Rev. 2001, 101, 2921-2990.

(58) Percec, V.; Guliashvili, T.; Ladislaw, J. S.; Wistrand, A.; Stjerndahl, A.; Sienkowska, M. J.; Monteiro, M. J.; Sahoo, S. J. Am. Chem. Soc. 2006, 128, 14156-14165.

(59) Siegwart, D. J.; Oh, J. K.; Matyjaszewski, K. Prog. Polym. Sci. 2012, 37, 18-37.

(60) Nicolas, J.; Mantovani, G.; Haddleton, D. M. Macromol. Rapid Commun. 2007, 28, 1083-1111.

(61) Xia, Y.; Yin, X.; Burke, N. a D.; Sto, H. D. H. Macromolecules 2005, 5937-5943.

(62) Tsarevsky, N. V.; Pintauer, T.; Matyjaszewski, K. Macromolecules 2004, 37, 9768-9778.

(63) Zhang, Q.; Wilson, P.; Li, Z.; McHale, R.; Godfrey, J.; Anastasaki, A.; Waldron, C.; Haddleton, D. M. J. Am. Chem. Soc. 2013, 135, 7355-7363.

(64) Hu, Y.; Darcos, V.; Monge, S.; Li, S.; Zhou, Y.; Su, F. J. Mater. Chem. B 2014, 2, 27382748.

(65) Li, Z.; Guo, X.; Matsushita, S.; Guan, J. Biomaterials 2011, 32, 3220-3232.

(66) De Graaf, A. J.; Boere, K. W. M.; Kemmink, J.; Fokkink, R. G.; Van Nostrum, C. F.; Rijkers, D. T. S.; Van Der Gucht, J.; Wienk, H.; Baldus, M.; Mastrobattista, E.; Vermonden, T.; Hennink, W. E. Langmuir 2011, 27, 9843-9848.

(67) De Graaf, A. J.; Azevedo Próspero Dos Santos, I. I.; Pieters, E. H. E.; Rijkers, D. T. S.; Van Nostrum, C. F.; Vermonden, T.; Kok, R. J.; Hennink, W. E.; Mastrobattista, E. J. Control. Release 2012, 162, 582-590.

(68) Kim, K. H.; Kim, J.; Jo, W. H. Polymer 2005, 46, 2836-2840.

(69) Jin, S.; Liu, M.; Chen, S.; Gao, C. Eur. Polym. J. 2008, 44, 2162-2170. 
(70) Boyer, C.; Bulmus, V.; Davis, T. P.; Ladmiral, V.; Liu, J.; Perrier, S. Chem. Rev. 2009, 109, 5402-5436.

(71) Chong, B. Y. K.; Krstina, J.; Le, T. P. T.; Moad, G.; Postma, A.; Rizzardo, E.; Thang, S. H. Macromolecules 2003, 36, 2256-2272.

(72) Convertine, A. J.; Lokitz, B. S.; Vasileva, Y.; Myrick, L. J.; Scales, C. W.; Lowe, A. B.; McCormick, C. L. Macromolecules 2006, 39, 1724-1730.

(73) Hoyle, C. E.; Bowman, C. N. Angew. Chemie Int. Ed. 2010, 49, 1540-1573.

(74) Li, H.; Yu, B.; Matsushima, H.; Hoyle, C. E.; Lowe, A. B. Macromolecules 2009, 42, 65376542.

(75) Roth, P. J.; Boyer, C.; Lowe, A. B.; Davis, T. P. Macromol. Rapid Commun. 2011, 32, 1123-1143.

(76) Stubbs, M.; McSheehy, P. M. .; Griffiths, J. R.; Bashford, C. L. Mol. Med. Today 2000, 6, 15-19.

(77) Estrella, V.; Chen, T.; Lloyd, M.; Wojtkowiak, J.; Cornnell, H. H.; Ibrahim-Hashim, A.; Bailey, K.; Balagurunathan, Y.; Rothberg, J. M.; Sloane, B. F.; Johnson, J.; Gatenby, R. A.; Gillies, R. J. Cancer Res. 2013, 73, 1524-1535.

(78) Vasir, J.; Labhasetwar, V. Adv. Drug Deliv. Rev. 2007, 59, 718-728.

(79) Fröhlich, E. Int. J. Nanomedicine 2012, 7, 5577-5591.

(80) Scott, C. C.; Vacca, F.; Gruenberg, J. Semin. Cell Dev. Biol. 2014, 31, 2-10.

(81) Kneipp, J.; Kneipp, H.; Wittig, B.; Kneipp, K. J. Phys. Chem. C 2010, 114, 7421-7426.

(82) Schmaljohann, D. Adv. Drug Deliv. Rev. 2006, 58, 1655-1670.

(83) Huang, C.-J.; Chang, F.-C. Macromolecules 2008, 41, 7041-7052.

(84) Chen, J.; Liu, M.; Gong, H.; Cui, G.; Lü, S.; Gao, C.; Huang, F.; Chen, T.; Zhang, X.; Liu, Z. Polym. Chem. 2013, 4, 1815-1825.

(85) Saito, G.; Swanson, J. A.; Lee, K.-D. Adv. Drug Deliv. Rev. 2003, 55, 199-215.

(86) Meng, F.; Hennink, W. E.; Zhong, Z. Biomaterials 2009, 30, 2180-2198.

(87) Brülisauer, L.; Gauthier, M. A.; Leroux, J.-C. J. Control. Release 2014, 195, 147-154.

(88) Vogt, A. P.; Sumerlin, B. S. Soft Matter 2009, 5, 2347-2351.

(89) Li, C.; Madsen, J.; Armes, S. P.; Lewis, A. L. Angew. Chemie - Int. Ed. 2006, 45, 35103513.

(90) Saha, K.; Agasti, S. S.; Kim, C.; Li, X.; Rotello, V. M. Chem. Rev. 2012, 112, 2739-2779.

(91) Thomas, V.; Namdeo, M.; Murali Mohan, Y.; Bajpai, S. K.; Bajpai, M. J. Macromol. Sci. Part A 2007, 45, 107-119.

(92) Kawano, T.; Niidome, Y.; Mori, T.; Katayama, Y.; Niidome, T. Bioconjug. Chem. 2009, 20, 209-212.

(93) Xu, H.; Xu, J.; Zhu, Z.; Liu, H.; Liu, S. Macromolecules 2006, 39, 8451-8455. 
(94) Morones, J. R.; Frey, W. J. Nanoparticle Res. 2010, 12, 1401-1414.

(95) Fernández-Barbero, A.; Suárez, I. J.; Sierra-Martín, B.; Fernández-Nieves, A.; de las Nieves, F. J.; Marquez, M.; Rubio-Retama, J.; López-Cabarcos, E. Adv. Colloid Interface Sci. 2009, 147-148, 88-108.

(96) Chomoucka, J.; Drbohlavova, J.; Huska, D.; Adam, V.; Kizek, R.; Hubalek, J. Pharmacol. Res. 2010, 62, 144-149.

(97) Wei, Q.; Ji, J.; Shen, J. Macromol. Rapid Commun. 2008, 29, 645-650.

(98) Jin, H.; Liu, X.; Gui, R.; Wang, Z. Colloids Surfaces B Biointerfaces 2015, 128, 498-505.

(99) Yang, H.-W.; Liu, H.-L.; Li, M.-L.; Hsi, I.-W.; Fan, C.-T.; Huang, C.-Y.; Lu, Y.-J.; Hua, M.-Y.; Chou, H.-Y.; Liaw, J.-W.; Ma, C.-C. M.; Wei, K.-C. Biomaterials 2013, 34, 5651-5660.

(100) Neuberger, T.; Schöpf, B.; Hofmann, H.; Hofmann, M.; von Rechenberg, B. J. Magn. Magn. Mater. 2005, 293, 483-496.

(101) Lee, N.; Yoo, D.; Ling, D.; Cho, M. H.; Hyeon, T.; Cheon, J. Chem. Rev. 2015, 115, 10637-10689.

(102) Hola, K.; Markova, Z.; Zoppellaro, G.; Tucek, J.; Zboril, R. Biotechnol. Adv. 2015, 33, 1162-1176.

(103) Hoare, T.; Santamaria, J.; Goya, G. F.; Irusta, S.; Lin, D.; Lau, S.; Padera, R.; Langer, R.; Kohane, D. S. Nano Lett. 2009, 9, 3651-3657.

(104) Pelton, R. H.; Chibante, P. Colloids and Surfaces 1986, 20, 247-256.

(105) Hoffman, A. S.; Stayton, P. S. Prog. Polym. Sci. 2007, 32, 922-932.

(106) Alemdaroglu, F. E.; Herrmann, A. Org. Biomol. Chem. 2007, 5, 1311-1320.

(107) Averick, S.; Mehl, R. A.; Das, S. R.; Matyjaszewski, K. J. Control. Release 2015, 205, 45-57.

(108) Harris, J. M.; Chess, R. B. Nat. Rev. Drug Discov. 2003, 2, 214-221.

(109) Pasut, G.; Veronese, F. M. J. Control. Release 2012, 161, 461-472.

(110) Dorresteijn, R.; Billecke, N.; Schwendy, M.; Pütz, S.; Bonn, M.; Parekh, S. H.; Klapper, M.; Müllen, K. Adv. Funct. Mater. 2014, 24, 4026-4033.

(111) Stayton, P. S.; Shimoboji, T.; Long, C.; Chilkoti, A.; Ghen, G.; Harris, J. M.; Hoffman, A. S. Nature 1995, 378, 472-474.

(112) Broyer, R. M.; Quaker, G. M.; Maynard, H. D. J. Am. Chem. Soc. 2008, 130, 1041-1047.

(113) Cate, M. G. J.; Rettig, H.; Bernhardt, K.; Bo, H. G. 2005, 38, 10643-10649.

(114) Liu, J.; Bulmus, V.; Herlambang, D. L.; Barner-Kowollik, C.; Stenzel, M. H.; Davis, T. P. Angew. Chemie 2007, 119, 3159-3163.

(115) Sumerlin, B. S. ACS Macro Lett. 2012, 1, 141-145.

(116) Chen, J. Biomaterials 1990, 11, 631-634.

(117) Tan, H.; Zhao, L.; Liu, W.; Ren, L.; Xu, S.; Chen, L.; Li, W. RSCAdv. 2014, 4, 60413-60420. 
(118) Lorenzo, M. M.; Decker, C. G.; Kahveci, M. U.; Paluck, S. J.; Maynard, H. D. Macromolecules 2016, 49, 30-37.

(119) Tan, H.; Zhao, L.; Liu, W.; Ren, L.; Xu, S.; Chen, L.; Li, W. RSC Adv. 2014, 4, 60413-60420.

(120) Heredia, K. L.; Bontempo, D.; Ly, T.; Byers, J. T.; Halstenberg, S.; Maynard, H. D. J. Am. Chem. Soc. 2005, 127, 16955-16960.

(121) Cummings, C.; Murata, H.; Koepsel, R.; Russell, A. J. Biomaterials 2013, 34, 74377443.

(122) Bontempo, D.; Maynard, H. D. J. Am. Chem. Soc. 2005, 127, 6508-6509.

(123) Boyer, C.; Bulmus, V.; Liu, J.; Davis, T. P.; Stenzel, M. H.; Barner-Kowollik, C. J. Am. Chem. Soc. 2007, 129, 7145-7154.

(124) De, P.; Li, M.; Gondi, S. R.; Sumerlin, B. S. J. Am. Chem. Soc. 2008, 130, 11288-11289.

(125) Li, M.; Li, H.; De, P.; Sumerlin, B. S. Macromol. Rapid Commun. 2011, 32, 354-359.

(126) Li, H.; Li, M.; Yu, X.; Bapat, A. P.; Sumerlin, B. S. Polym. Chem. 2011, 2, 1531-1535.

(127) Adams, D. J.; Topham, P. D. Soft Matter 2010, 6, 3707-3721.

(128) Deshayes, S.; Maurizot, V.; Clochard, M. C.; Baudin, C.; Berthelot, T.; Esnouf, S.; Lairez, D.; Moenner, M.; Déléris, G. Pharm. Res. 2011, 28, 1631-1642.

(129) Van Dijk, M.; Rijkers, D. T. S.; Liskamp, R. M. J.; Van Nostrum, C. F.; Hennink, W. E. Bioconjug. Chem. 2009, 20, 2001-2016.

(130) Molawi, K.; Studer, A. Chem. Commun. 2007, 5173-5175.

(131) Bulmus, V.; Patir, S.; Ali Tuncel, S.; Piskin, E. J. Appl. Polym. Sci. 2003, 88, 2012-2019.

(132) Kakwere, H.; Chun, C. K. Y.; Jolliffe, K. a; Payne, R. J.; Perrier, S. Chem. Commun. 2010, 46, 2188-2190.

(133) Couet, J.; Samuel, J. D. J. S.; Kopyshev, A.; Santer, S.; Biesalski, M. Angew. Chemie Int. Ed. 2005, 44, 3297-3301.

(134) Danial, M.; Tran, C. M.-N.; Jolliffe, K. A.; Perrier, S. J. Am. Chem. Soc. 2014, 136, 80188026.

(135) Trzebicka, B.; Robak, B.; Trzcinska, R.; Szweda, D.; Suder, P.; Silberring, J.; Dworak, A. Eur. Polym. J. 2013, 49, 499-509.

(136) De Graaf, A. J.; Mastrobattista, E.; Vermonden, T.; Van Nostrum, C. F.; Rijkers, D. T. S.; Liskamp, R. M. J.; Hennink, W. E. Macromolecules 2012, 45, 842-851.

(137) Benassi, M. S.; Gamberi, G.; Magagnoli, G.; Molendini, L.; Ragazzini, P.; Merli, M.; Chiesa, F.; Balladelli, A.; Manfrini, M.; Bertoni, F.; Mercuri, M.; Picci, P. Ann. Oncol. 2001, 12, 75-80.

(138) Hentschel, J.; Bleek, K.; Ernst, O.; Bo, H. G. Macromolecules 2008, 41, 1073-1075.

(139) McQuade, D. T.; Pullen, A. E.; Swager, T. M. Chem. Rev. 2000, 100, 2537-2574.

(140) Peterson, A. M.; Heemstra, J. M. Wiley Interdiscip. Rev. Nanomedicine 
Nanobiotechnology 2015, 7, 282-297.

(141) Guo, W.; Lu, C. H.; Qi, X. J.; Orbach, R.; Fadeev, M.; Yang, H. H.; Willner, I. Angew. Chemie - Int. Ed. 2014, 53, 10134-10138.

(142) Umeno, D.; Kawasaki, M.; Maeda, M. Bioconjug. Chem. 1998, 9, 719-724.

(143) Umeno, D.; Maeda, M. Anal. Sci. 1997, 13, 553-556.

(144) Pan, P.; Fujita, M.; Ooi, W.-Y.; Sudesh, K.; Takarada, T.; Goto, A.; Maeda, M. Langmuir 2012, 28, 14347-14356.

(145) Mori, T.; Umeno, D.; Maeda, M. Biotechnol. Bioeng. 2001, 72, 261-268.

(146) Miyamoto, D.; Tang, Z.; Takarada, T.; Maeda, M. Chem. Commun. 2007, 4743-4745.

(147) Isoda, K.; Kanayama, N.; Miyamoto, D.; Takarada, T.; Maeda, M. React. Funct. Polym. 2011, 71, 367-371.

(148) Crocker, J. C.; Matteo, J. A.; Dinsmore, A. D.; Yodh, A. G. Phys. Rev. Lett. 1999, 82, 4352-4355.

(149) Yeh, M.-K.; Hsin-I Chang; Ming-Yen Cheng. Int. J. Nanomedicine 2011, 7, 49-60.

(150) Allen, T. M.; Cullis, P. R. Adv. Drug Deliv. Rev. 2013, 65, 36-48.

(151) Gerasimov, O. V.; Boomer, J. a.; Qualls, M. M.; Thompson, D. H. Adv. Drug Deliv. Rev. 1999, 38, 317-338.

(152) Huang, S.-L. Adv. Drug Deliv. Rev. 2008, 60, 1167-1176.

(153) Ta, T.; Porter, T. M. J. Control. Release 2013, 169, 112-125.

(154) Kono, K.; Hayashi, H.; Takagishi, T. J. Control. Release 1994, 30, 69-75.

(155) Kono, K. Adv. Drug Deliv. Rev. 2001, 53, 307-319.

(156) Papahadjopoulos, D.; Jacobson, K.; Nir, S.; Isac, I. BBA - Biomembr. 1973, 311, 330348.

(157) Yatvin, M.; Weinstein, J.; Dennis, W.; Blumenthal, R. Science 1978, 202, 1290-1293.

(158) Kono, K.; Murakami, T.; Yoshida, T.; Haba, Y.; Kanaoka, S.; Takagishi, T.; Aoshima, S. Bioconjug. Chem. 2005, 16, 1367-1374.

(159) Han, H.; Shin, B.; Choi, H. Eur. J. Pharm. Biopharm. 2006, 62, 110-116.

(160) Yamada, N.; Okano, T.; Sakai, H.; Karikusa, F.; Sawasaki, Y.; Sakurai, Y. Die Makromol. Chemie, Rapid Commun. 1990, 11, 571-576.

(161) Okano, T.; Yamada, N.; Sakai, H.; Sakurai, Y. J. Biomed. Mater. Res. 1993, 27, $1243-$ 1251.

(162) Kushida, A.; Yamato, M.; Konno, C.; Kikuchi, A.; Sakurai, Y.; Okano, T. J. Biomed. Mater. Res. 2000, 51, 216-223.

(163) Schmaljohann, D.; Oswald, J.; Jørgensen, B.; Nitschke, M.; Beyerlein, D.; Werner, C. Biomacromolecules 2003, 4, 1733-1739.

(164) Schmaljohann, D.; Beyerlein, D.; Nitschke, M.; Werner, C. Langmuir 2004, 20, 10107- 
10114.

(165) Tillet, G.; Boutevin, B.; Ameduri, B. Prog. Polym. Sci. 2011, 36, 191-217.

(166) Hoare, T. R.; Kohane, D. S. Polymer 2008, 49, 1993-2007.

(167) Talelli, M.; Barz, M.; Rijcken, C. J. F.; Kiessling, F.; Hennink, W. E.; Lammers, T. Nano Today 2015, 10, 93-117.

(168) Hennink, W. E.; van Nostrum, C. F. Adv. Drug Deliv. Rev. 2012, 64, 223-236.

(169) Lee, K. Y.; Mooney, D. J. Chem. Rev. 2001, 101, 1869-1880.

(170) Hoffman, A. S. Ann. N. Y. Acad. Sci. 2001, 944, 62-73.

(171) Vermonden, T.; Censi, R.; Hennink, W. E. Chem. Rev. 2012, 112, 2853-2888.

(172) Qiu, Y.; Park, K. Adv. Drug Deliv. Rev. 2012, 64, 49-60.

(173) Klouda, L. Eur. J. Pharm. Biopharm. 2015, 97, 338-349.

(174) Cheng, R.; Feng, F.; Meng, F.; Deng, C.; Feijen, J.; Zhong, Z. J. Control. Release 2011, 152, 2-12.

(175) Li, Y.; Maciel, D.; Rodrigues, J.; Shi, X.; Tomás, H. Chem. Rev. 2015, 115, 8564-8608.

(176) Otake, K.; Inomata, H.; Konno, M.; Saito, S. Macromolecules 1990, 23, 283-289.

(177) Yoshida, R.; Uchida, K.; Kaneko, Y.; Sakai, K.; Kikuchi, A.; Sakurai, Y.; Okano, T. Nature 1995, 374, 240-242.

(178) Varga, I.; Gilányi, T.; Mészáros, R.; Filipcsei, G.; Zrínyi, M. J. Phys. Chem. B 2001, 105, 9071-9076.

(179) Hu, B.-H.; Su, J.; Messersmith, P. B. Biomacromolecules 2009, 10, 2194-2200.

(180) Boere, K. W. M.; Soliman, B. G.; Rijkers, D. T. S.; Hennink, W. E.; Vermonden, T. Macromolecules 2014, 47, 2430-2438.

(181) Boere, K. W. M.; van den Dikkenberg, J.; Gao, Y.; Visser, J.; Hennink, W. E.; Vermonden, T. Biomacromolecules 2015, 16, 2840-2851.

(182) Tan, H.; Ramirez, C. M.; Miljkovic, N.; Li, H.; Rubin, J. P.; Marra, K. G. Biomaterials 2009, 30, 6844-6853.

(183) Campbell, S. B.; Patenaude, M.; Hoare, T. Biomacromolecules 2013, 14, 644-653.

(184) Zhang, B.; He, W.-D.; Li, L.; Sun, X.; Li, W.; Zhang, K. J. Polym. Sci. Part A Polym. Chem. 2010, 48, 3604-3612.

(185) Galperin, A.; Long, T. J.; Ratner, B. D. Biomacromolecules 2010, 11, 2583-2592.

(186) Robb, S. A.; Lee, B. H.; McLemore, R.; Vernon, B. L. Biomacromolecules 2007, 8, 22942300 .

(187) Li, Y.; Xiao, K.; Zhu, W.; Deng, W.; Lam, K. S. Adv. Drug Deliv. Rev. 2014, 66, 58-73.

(188) Wei, H.; Cheng, S.-X.; Zhang, X.-Z.; Zhuo, R.-X. Prog. Polym. Sci. 2009, 34, 893-910.

(189) van Nostrum, C. F. Soft Matter 2011, 7, 3246-3259.

(190) Read, E. S.; Armes, S. P. Chem. Commun. 2007, 3021-3035. 
(191) Bronich, T. K.; Keifer, P. A.; Shlyakhtenko, L. S.; Kabanov, A. V. J. Am. Chem. Soc. 2005, $127,8236-8237$.

(192) Kim, S. Y.; Lee, Y. M. Biomaterials 2001, 22, 1697-1704.

(193) Thurmond, K. B.; Kowalewski, T.; Wooley, K. L. J. Am. Chem. Soc. 1996, 118, 72397240.

(194) Li, Y.; Lokitz, B. S.; McCormick, C. L. Macromolecules 2006, 39, 81-89.

(195) Li, Y.; Lokitz, B. S.; Armes, S. P.; McCormick, C. L. Macromolecules 2006, 39, 27262728.

(196) Li, L.-Y.; He, W.-D.; Li, J.; Zhang, B.-Y.; Pan, T.-T.; Sun, X.-L.; Ding, Z.-L. Biomacromolecules 2010, 11, 1882-1890.

(197) Reddy, N.; Reddy, R.; Jiang, Q. Trends Biotechnol. 2015, 33, 362-369.

(198) Yuan, J. J.; Mykhaylyk, O. O.; Ryan, A. J.; Armes, S. P. J. Am. Chem. Soc. 2007, 129, 1717-1723.

(199) Wei, H.; Cheng, C.; Chang, C.; Chen, W. Q.; Cheng, S.; Zhang, X. Z.; Zhuo, R. X. Langmuir 2008, 24, 4564-4570.

(200) Jiang, X.; Liu, S.; Narain, R. Langmuir 2009, 25, 13344-13350.

(201) Zhang, J.; Zhou, Y.; Zhu, Z.; Ge, Z.; Liu, S. Macromolecules 2008, 41, 1444-1454.

(202) Zhou, X.; Li, J.; Wu, C.; Zheng, B. Macromol. Rapid Commun. 2008, 29, 1363-1367. 



\section{Chapter 3}

\section{Native chemical ligation for crosslinking of flower-like micelles}

Marzieh Najafi ${ }^{1}$, Neda Kordalivand ${ }^{1}$, Mohammad-Amin Moradi ${ }^{2,3}$, Joep van den Dikkenberg ${ }^{1}$, Remco Fokkink ${ }^{4}$, Heiner Friedrich ${ }^{2,3}$, Nico A. J. M. Sommerdijk ${ }^{2,3}$, Mathew Hembury ${ }^{1}$, Tina Vermonden ${ }^{1}$

${ }^{1}$ Department of Pharmaceutics, Utrecht Institute for Pharmaceutical Sciences (UIPS), Science for Life, Faculty of Science, Utrecht University, P.O. Box 80082, 3508 TB Utrecht, The Netherlands.

${ }^{2}$ Laboratory of Materials and Interface Chemistry \& Centre for Multiscale Electron Microscopy Department of Chemical Engineering and Chemistry, Eindhoven University of Technology, Eindhoven $5600 \mathrm{MB}$, The Netherlands

${ }^{3}$ Institute for Complex Molecular Systems, Eindhoven University of Technology, Eindhoven $5600 \mathrm{MB}$, The Netherlands

${ }_{4}^{4}$ Physical Chemistry and Soft Matter, Wageningen University \& Research, Stippeneng 4, 6708 WE Wageningen, The Netherlands. 


\section{Abstract}

In this study, native chemical ligation (NCL) was used as a selective crosslinking method to form core-crosslinked thermosensitive polymeric micelles for drug delivery applications. To this end, two complementary ABA triblock copolymers having polyethylene glycol (PEG) as midblock were synthesized by atom transfer radical polymerization (ATRP). The thermosensitive poly isopropyl acrylamide (PNIPAM) outer blocks of the polymers were copolymerized with either $\mathrm{N}$-(2-hydroxypropyl)methacrylamide-cysteine (HPMA-Cys), P(NIPAM-co-HPMA-Cys)-PEG-P(NIPAM-co-HPMA-Cys) (PNC), or N-(2-hydroxypropyl) methacrylamide-ethylthioglycolate succinic acid (HPMA-ETSA), P(NIPAM-co-HPMA-ETSA) PEG-P(NIPAM-CO-HPMA-ETSA) (PNE). Mixing of these polymers in aqueous solution followed by heating to $50{ }^{\circ} \mathrm{C}$ resulted in the formation of thermosensitive flower-like micelles. Subsequently, native chemical ligation in the core of micelles resulted in stabilization of the micelles with a Z-average of $65 \mathrm{~nm}$ at body temperature. Decreasing the temperature to $10^{\circ} \mathrm{C}$ only affected the size of the micelles (increased to $90 \mathrm{~nm}$ ) but hardly affected the polydispersity index (PDI) and aggregation number ( $\left.N_{a g g}\right)$ confirming covalent stabilization of the micelles by NCL. CryoTEM images showed micelles with an uniform spherical shape and dark patches close to the corona of micelles were observed in the tomographic view. The dark patches represent more dense areas in the micelles which coincide with the higher content of HPMA-Cys/ETSA close to the PEG chain revealed by the polymerization kinetics study. Notably, this crosslinking method provides the possibility for conjugation of functional molecules either by using the thiol moieties still present after NCL or by simply adjusting the molar ratio between the polymers (resulting in excess cysteine or thioester moieties) during micelle formation. Furthermore, in vitro cell experiments demonstrated that fluorescently labeled micelles were successfully taken up by HeLa cells while cell viability remained high even at high micelle concentrations. These results demonstrate the potential of these micelles for drug delivery applications. 


\section{Introduction}

Polymeric micelles (PM) can be formed by amphiphilic block copolymers and have been studied extensively to improve delivery of mainly hydrophobic drugs ${ }^{1,2}$. In aqueous media, at concentrations above the critical micelle concentration (CMC), amphiphilic block copolymers $(A B$ or $A B A)$ self-assemble into nano-sized particles. While $A B$ polymers selfassemble into star-like micelles, $A B A$ triblock copolymers with a hydrophilic midblock (B) are capable of self-assembling into so-called flower-like micelles ${ }^{3,4,5}$. Although flower-like micelles share many characteristics with star-like micelles, they have been reported to have some advantageous properties regarding lower CMC and higher stability ${ }^{6,7,8}$.

The hydrophilic midblock (B block) in flower-like micelles is often composed of poly (ethylene glycol) (PEG) ${ }^{6}$. Starting from this PEG midblock, there are several methods to synthesize ABA block copolymers among which atom transfer radical polymerization (ATRP) provides good control over the structure and sequence of monomers in a polymer ${ }^{9,10}$. ATRP conditions enable the growth of hydrophobic A-blocks with a desired length and tunable monomer composition ${ }^{11}$.

During micellization of ABA block polymers, in aqueous solution, the PEG block forms a looped structure of the hydrophilic corona surrounding the hydrophobic core composed of the self-assembled outer blocks ${ }^{3}$. The hydrophobic core has been used to solubilize hydrophobic drugs and the hydrophilic shell stabilizes micelles and enhances their circulation time in body fluids ${ }^{12,13}$. However, the equilibrium between micelles and unimers is highly affected by dilution and at concentrations below the $\mathrm{CMC}$, micelles start to dissociate. This dissociation can lead to premature release of a loaded drug from the micelles upon administration in e.g. the circulation ${ }^{14}$. Moreover, proteins present in the body can extract drugs from micellar formulations. To enhance the in vivo stability of micelles several methods have been developed, including the frequently applied method of core crosslinking and covalent linking of drugs to the polymers present in the micelles ${ }^{15}$. For covalent crosslinking of the micellar core, the reactive groups should be located in the hydrophobic block of the polymers. Of course, it is important that the chemical properties of these functional groups do not hamper micelle formation. A commonly applied method for core crosslinking of micelles makes use of radical polymerization. In this method, polymerizable groups such as methacrylates or acrylates are introduced in the hydrophobic block of amphiphilic polymers, which are located in the core of the micelles after micellization. Subsequently, by UV illumination in the presence of a photoinitiator or by thermal radical polymerization, these functional groups can be crosslinked in the core of this type of micelles ${ }^{16,8}$. Another common method for covalent crosslinking of micelles is introducing reactive groups, such as, epoxy or acidic moieties in the side chain of 
amphiphilic polymers that are subsequently crosslinked by a bifunctional molecule, such as a diamine ${ }^{17,18}$. Also, disulfide bridges as crosslinks can be introduced by using cystamine in the micellar core, which gives the micelles triggered release properties interesting for compounds that need to be delivered intracellularly. These disulfide bridges will be broken in the intracellular reducing environment ${ }^{19,20}$. Also, free thiols in the hydrophobic block of the amphiphilic polymer can be used for disulfide core crosslinking, however, oxidative conditions should be provided ${ }^{21,22}$. Recently, 'click' reactions received attention for crosslinking of polymeric micelles ${ }^{23,24}$. Several studies reported the formation of crosslinked micelles and nanoparticles using copper-catalyzed alkyne-azide cycloaddition reaction $(\mathrm{CUAAC})^{25,26}$. For example, redox-responsive core crosslinked micelles were synthesized using bis-(azidoethyl) disulfide as a redox-responsive cross-linker ${ }^{27}$. Other studies used Diels-Alder reactions for crosslinking, e.g. formation of star-like micelles by crosslinking block copolymers having furan functionalities using bismaleimide crosslinkers in tetrahydrofuran (THF $)^{28}$. Furthermore, PEG-polyester type polymers with pending mercapto groups were crosslinked via a thiol-ene 'click' chemistry approach using a diacrylate crosslinker ${ }^{29}$.

Besides all the developments in this field, many of the applied crosslinking methods suffer from some limitations (e.g. exposure to radicals, oxidative conditions, use of (toxic) catalysts which mostly require an inert environment, or use of organic solvents), that can potentially damage the cargo of the micelles. Furthermore, the functional groups used in these methods will be consumed during crosslinking and cannot be used for further modification steps. Therefore, introducing a crosslinking method that is not only applicable in aqueous solutions but also retains its reactive groups in the micellar core after crosslinking is of high interest. Among chemical conjugation methods performed in aqueous solution, native chemical ligation ${ }^{30}$, a chemoselective method, has been studied for crosslinking of hydrogels ${ }^{31,32,33}$. However, this crosslinking method has not been applied yet for corecrosslinking of micelles. Native chemical ligation involves nucleophilic attack of the thiol group of an $\mathrm{N}$-terminal cysteine to a thioester moiety. The obtained thioester intermediate rearranges by an intramolecular $\mathrm{S}, \mathrm{N}$-acyl shift that results in the formation of an amide. Notably, after the $S, N$-acyl shift a free thiol moiety remains available, which offers the possibility to further conjugate desirable molecules via e.g. a disulfide bond.

The goal of this study was to investigate native chemical ligation as a selective reaction that occurs in water for core crosslinking of flower-like micelles ${ }^{34}$. For this purpose, an ABA triblock copolymer consisting of polyethylene glycol (PEG) as midblock and thermosensitive poly $\mathrm{N}$-isopropyl acrylamide (NIPAM)-co- $N$-(2-hydroxypropyl) methacrylamide-cysteine (HPMA-Cys) as outer blocks P(NIPAM-co-HPMA-Cys)-PEG- P(NIPAM-co-HPMA-Cys) (PNC)) was synthesized by ATRP. A complementary polymer, P(NIPAM-co-HPMA-ETSA)-PEG- 
P(NIPAM-Co-HPMA-ETSA) (PNE), containing a novel monomer of $\mathrm{N}$-(2hydroxypropyl)methacrylamide-ethylthioglycolate succinic acid (HPMA-ETSA) carrying a thioester moiety, was developed. In these amphiphilic polymers designed to self-assemble into micelles, the PEG block represents the hydrophilic part, PNIPAM blocks initiate micellization in aqueous solution by increasing temperature and HPMA-Cys/ETSA monomers provide $\mathrm{N}$-terminal cysteine and thioester functionalities for NCL cross-linking to stabilize the micellar structure. Upon mixing of the polymers in aqueous solution, the presence of PNIPAM thermosensitive blocks allows micelle formation by increasing the temperature above the lower critical solution temperature (LCST) of these polymers ${ }^{35}$. The stability of the micelles after covalent cross-linking by NCL was investigated by lowering the temperature of the micellar solution below the LCST of the polymers. In addition, the shape and conformation of micelles were studied by static light scattering and CryoTEM. To investigate cytocompatibility for potential biomedical applications of the obtained micelles, a cytotoxicity assay was performed. Moreover, cellular uptake of labeled micelles was studied using A549 (human lung carcinoma cell line) and HeLa (human epithelial cervix carcinoma cell line) cells.

\section{Materials and Methods}

All commercial chemicals were obtained from Sigma-Aldrich (Zwijndrecht, The Netherlands) and used as received unless indicated otherwise. $N$-(2-hydroxypropyl)methacrylamide (HPMA) was synthesized by a reaction of methacryloyl chloride with 1-aminopropan-2-ol in dichloromethane according to a literature procedure ${ }^{36}$. Peptide grade dichloromethane $\left(\mathrm{CH}_{2} \mathrm{Cl}_{2}\right)$ was obtained from Biosolve (Valkenswaard, The Netherlands). N,N'dimethylaminopyridine (DMAP) was purchased from Fluka (Zwijndrecht, The Netherlands). Boc-S-acetamidomethyl-L-cysteine (Boc-Cys(Acm)-OH was purchased from Bachem (Bubendorf, Switzerland). N-(2-hydroxypropyl)methacrylamide-Boc-S-acetamidomethyl-Lcysteine (HPMA-Boc-Cys (Acm)) was synthesized as described by Boere et $\mathrm{al}^{33}$. Ethylthioglycolate succinic acid (ETSA) was prepared according to a literature procedure ${ }^{31}$. Phosphate buffered saline (PBS) pH $7.4\left(8.2 \mathrm{~g} / \mathrm{L} \mathrm{NaCl}, 3.1 \mathrm{~g} / \mathrm{L} \mathrm{Na} \mathrm{HPO}_{4} \cdot 12 \mathrm{H}_{2} \mathrm{O}, 0.3 \mathrm{~g} / \mathrm{L}\right.$ $\mathrm{NaH}_{2} \mathrm{PO}_{4} \cdot 2 \mathrm{H}_{2} \mathrm{O}$ ) was purchased from B. Braun (Melsungen, Germany). Celltiter AQ-MTS (3(4,5-dimethylthiazol-2-yl)- 5-(3-carboxymethoxyphenyl)-2-(4-sulfophenyl)-2H-tetrazolium) \#3580 and Lysis Solution \#G182A were purchased from Promega (USA). NHS-Alexa Fluor $^{\circledR} 647$ and maleimide-Alexa flour C5 568 fluorescent dyes were obtained from Invitrogen (Eugene, OR, USA).

\section{Synthesis of poly(ethylene glycol) bis(2-bromoisobutyrate)}


The synthesis of a PEG macroinitiator was achieved according to a method previously reported ${ }^{3}$ with slight modifications. Briefly, dried PEG $6 \mathrm{kDa}$ (5 gr) was dissolved in $70 \mathrm{~mL}$ of dry THF and purged with nitrogen. To this solution, triethylamine $(0.7 \mathrm{~mL})$ and $\alpha-$ bromoisobutyryl bromide $(0.6 \mathrm{~mL})$ were added and the reaction mixture was stirred overnight at room temperature. Next, the formed bromide salt was filtered off and subsequently, THF was evaporated under reduced pressure. The crude product was dialyzed against water for 2 days and afterwards lyophilized. The degree of functionalization was determined by addition of trichloroacetyl isocyanate (TAIC) reacting with unmodified $\mathrm{OH}$ groups ${ }^{37} .{ }^{1} \mathrm{H}-\mathrm{NMR}$ analysis confirmed the formation of a fully functionalized PEG ATRP macroinitiator. ${ }^{1} \mathrm{H}-\mathrm{NMR}\left(\mathrm{CDCl}_{3}\right): \delta=4.3\left(\mathrm{t}, 4 \mathrm{H}, \mathrm{OCH}_{2}\right), 3.85\left(\mathrm{t}, 4 \mathrm{H}, \mathrm{OCH}_{2}\right), 3.65(\mathrm{t}, 531 \mathrm{H}$, $\left.\mathrm{OCH}_{2}\right), 3.35\left(\mathrm{t}, 4 \mathrm{H}, \mathrm{OCH}_{2}\right), 1.85 \mathrm{ppm}\left(\mathrm{s}, 12 \mathrm{H}, \mathrm{CCH}_{3}\right)$.

\section{Synthesis of $\mathrm{N}$-(2-hydroxypropyl) methacrylamide-ethylthioglycolate succinic acid (HPMA-ETSA)}

In a typical procedure, ethylthioglycolate succinic acid (ETSA) (1.54 g, $7 \mathrm{mmol})$, HPMA (1.00 $\mathrm{g}, 7 \mathrm{mmol}$ ), and DMAP (85 mg, $7 \mu \mathrm{mol}$ ) were dissolved in dry $\mathrm{CH}_{2} \mathrm{Cl}_{2}(7 \mathrm{~mL})$. To this solution, $N, N^{\prime}$-dicyclohexylcarbodiimide (DCC) $(1.44 \mathrm{~g}, 7 \mathrm{mmol}$ ) was added, and the reaction mixture was stirred for $16 \mathrm{~h}$ under a nitrogen atmosphere at room temperature. Subsequently, the suspension was cooled to $0{ }^{\circ} \mathrm{C}$ and filtered, after which the filtrate was concentrated. The crude product was purified by silica gel chromatography, using $\mathrm{CH}_{2} \mathrm{Cl}_{2} / \mathrm{CH}_{3} \mathrm{OH}(9: 1 \mathrm{v} / \mathrm{v})$ as eluent. The monomer HPMA-ETSA was obtained as milky oil with a yield of $57 \%$. ${ }^{1} \mathrm{H}-\mathrm{NMR}$ $\left(\mathrm{CDCl}_{3}\right): \delta=6.28(\mathrm{~s}, 1 \mathrm{H}, \mathrm{NH}), 5.68\left(\mathrm{~s}, 1 \mathrm{H}, \mathrm{H}_{2} \mathrm{C}=\mathrm{CH}\right), 5,31\left(\mathrm{~s}, 1 \mathrm{H}, \mathrm{H}_{2} \mathrm{C}=\mathrm{CH}\right), 5.05(\mathrm{~m}, 1 \mathrm{H}$, $\mathrm{CH}_{2} \mathrm{CH}\left(\mathrm{CH}_{3}\right) \mathrm{O}$ ), 4.16 (q, 2H, $\left.\mathrm{CH}_{3} \mathrm{CH}_{2} \mathrm{O}\right), 3.67$ (d, $2 \mathrm{H}, \mathrm{C}(\mathrm{O}) \mathrm{SCH}_{2} \mathrm{C}(\mathrm{O})$ ), 3.56 (dd, $1 \mathrm{H}$, $\mathrm{NHCH}_{2} \mathrm{CH}\left(\mathrm{CH}_{3}\right) \mathrm{O}$ ), 3.32 (dd, $1 \mathrm{H}, \mathrm{NHCH}_{2} \mathrm{CH}\left(\mathrm{CH}_{3}\right) \mathrm{O}$ ), 2.95 (t, 2H, $\mathrm{SC}(\mathrm{O}) \mathrm{CH}_{2} \mathrm{CH}_{2}$ ), 2.63 (t, $2 \mathrm{H}$, $\left.\mathrm{SC}(\mathrm{O}) \mathrm{CH}_{2} \mathrm{CH}_{2}\right), 1.94\left(\mathrm{~s}, 3 \mathrm{H}, \mathrm{C}=\mathrm{C}\left(\mathrm{CH}_{3}\right)\right), 1.24\left(\mathrm{~m}, 6 \mathrm{H}, \mathrm{CH}_{3} \mathrm{CH}_{2} \mathrm{O}\right)$ and $\left.\mathrm{NHCH}_{2} \mathrm{CH}\left(\mathrm{CH}_{3}\right) \mathrm{O}\right) .{ }^{13} \mathrm{C}-\mathrm{NMR}$ $\left(\mathrm{CDCl}_{3}\right): \delta=196.5,172.3,171.3,168.5,139.8,119.5,70.7,61.9,44.0,38.2,31.2,29.4,18.9$, 17.5, 14.0 (SI-Fig. 1).

\section{Synthesis of P(NIPAM-co-HPMA-ETSA)-PEG-P(NIPAM-co-HPMA-ETSA), PNE}

Poly(ethylene glycol) bis(2-bromoisobutyrate) (50 mg, $7.9 \mu \mathrm{mol}$ ), CuBr (4.5 mg, 0.031 mmol), $\mathrm{CuBr}_{2}$ (4.7 mg, $0.021 \mathrm{mmol}$ ), NIPAM (268.9 mg; $2.3 \mathrm{mmol}$ ) and HPMA-ETSA (55 mg, $0.16 \mathrm{mmol}$ ) were dissolved in a mixture of $2.5 \mathrm{~mL}$ water and $2 \mathrm{~mL}$ ethanol. The mixture was stirred and deoxygenated by flushing with nitrogen for 15 minutes at room temperature, then placed in an ice bath for another 15 minutes. After addition of $16 \mu \mathrm{L}(0.06 \mathrm{mmol})$ of tris[2-(dimethylamino)ethyl]amine (Me6TREN) to the solution, the color of the mixture 
immediately changed to blue and the reaction was left for 5 hours in an ice bath. The final product was dialyzed (cut off $10 \mathrm{kDa}$ ) against water at $4{ }^{\circ} \mathrm{C}$ for one day and lyophilized. The obtained polymer was characterized by GPC and ${ }^{1} \mathrm{H}-\mathrm{NMR}$ (SI-Fig. 2, 4A).

\section{Synthesis of P(NIPAM-co-HPMA-Cys)-PEG-P(NIPAM-co-HPMA-Cys), PNC}

Poly(ethylene glycol) bis(2-bromoisobutyrate) (50 mg, $7.9 \mu \mathrm{mol}$ ), $\mathrm{CuBr}(4.5 \mathrm{mg}, 0.031$ $\mathrm{mmol}$ ), $\mathrm{CuBr}_{2}$ (4.7 mg, $0.021 \mathrm{mmol}$ ), NIPAM (268.9 mg; $2.4 \mathrm{mmol}$ ) and HPMA-Boc-Cys(Acm) $(67 \mathrm{mg}, 0.16 \mathrm{mmol}$ ) were dissolved in a mixture of $3.5 \mathrm{ml}$ of water and acetonitrile with a ratio of 3:1. The mixture was stirred and deoxygenated by flushing with nitrogen for 15 minutes at room temperature, then placed in an ice bath for another 15 minutes. After addition of $16 \mu \mathrm{L}(0.06 \mathrm{mmol})$ of Me 6 TREN to the solution, the color of the mixture immediately changed to blue and the reaction was left stirring for two hours in an ice bath. The final product was dialyzed (cut off $10 \mathrm{kDa}$ ) against water at $4{ }^{\circ} \mathrm{C}$ for one day and subsequently lyophilized. The obtained polymer was characterized by GPC and ${ }^{1} \mathrm{H}-\mathrm{NMR}$. To replace the bromide end groups, the obtained polymer was dissolved in $5 \mathrm{~mL} \mathrm{CH} \mathrm{Cl}_{2}$ and treated with an excess $(500 \mu \mathrm{L})$ of mercaptoethanol for $24 \mathrm{~h}$. Next, the solvent was concentrated by evaporation and the pure polymer was obtained after precipitation in cold diethyl ether. Finally, the Acm and Boc protecting groups of cysteine were removed as explained previously ${ }^{32}$. The obtained polymer was characterized by GPC and ${ }^{1} \mathrm{H}-\mathrm{NMR}$ (SIFig. 3, 4B).

\section{Kinetics of polymerization}

At several time points, $250 \mu \mathrm{L}$ samples were taken from polymerization mixtures to monitor the conversion of polymerization by ${ }^{1} \mathrm{H}-\mathrm{NMR}$ and GPC. Samples of $50 \mu \mathrm{L}$ were diluted with air-saturated $\mathrm{CDCl}_{3}$ and analyzed by ${ }^{1} \mathrm{H}-\mathrm{NMR}$. The integrals of signals at 5.5 and $5.30 \mathrm{ppm}$ related to NIPAM and HPMA-Cys/ETSA respectively, were compared to the PEG signal at $3.65 \mathrm{ppm}$ as a reference. The remaining $200 \mu \mathrm{L}$ was dialyzed against water for one day and then lyophilized. The dried product was dissolved in $\mathrm{DMF} / \mathrm{LiCl}$ and the molecular weight was analyzed by GPC as described below.

\section{NMR spectroscopy}

The obtained monomers, polymers, and micelles were studied by ${ }^{1} \mathrm{H}-\mathrm{NMR}$ (400 MHz) and ${ }^{13} \mathrm{C}-\mathrm{NMR}(100 \mathrm{MHz})$ measured on an Agilent 400-MR NMR spectrometer (Agilent Technologies, Santa Clara, USA). The chemical shifts were calibrated against residual solvent 
peaks of $\mathrm{CDCl}_{3}(\delta=7.26 \mathrm{ppm}), \mathrm{D}_{2} \mathrm{O}(\delta=4.79 \mathrm{ppm})$ for ${ }^{1} \mathrm{H}-\mathrm{NMR}$, and $\delta=77.16 \mathrm{ppm}$ of $\mathrm{CDCl}_{3}$ for ${ }^{13} \mathrm{C}-\mathrm{NMR}$. Micelle solutions were analyzed on a Varian Inova $500 \mathrm{NMR}$ instrument (Varian Inc., California, USA) in $\mathrm{D}_{2} \mathrm{O}$.

\section{Gel permeation chromatography (GPC)}

To determine the molecular weight of polymers, GPC was performed on a Waters Alliance System (Waters Corporation, Milford, MA, USA) equipped with a refractive index detector using a Mixed-D column (Polymer Laboratories) at a temperature of $65^{\circ} \mathrm{C}$. The eluent consisting of $10 \mathrm{mM} \mathrm{LiCl}$ in DMF with a flow of $1 \mathrm{~mL} / \mathrm{min}$ was used as mobile phase and samples were prepared in the same solvent at a concentration of $5 \mathrm{mg} / \mathrm{mL}$. A series of linear PEGs with narrow and defined molecular weights were used as calibration standards.

\section{Determination of cloud point}

The cloud point (CP) of the obtained polymers was measured on a Jasco FP8300 spectrofluorometer (Tokyo, Japan) and excitation/emission wavelength was set at $650 \mathrm{~nm}$. Polymers were dissolved at a concentration of $1 \mathrm{mg} / \mathrm{mL}$ in PBS and heated from 10 to $50^{\circ} \mathrm{C}$ with a heating rate of $1{ }^{\circ} \mathrm{C} / \mathrm{min}$. The $\mathrm{CP}$ was defined as the onset of increasing scattering intensity.

\section{Micelle formation}

Micelles were formed by a fast heating method as follows: PNC and PNE were dissolved separately in PBS at a concentration of $3 \mathrm{mg} / \mathrm{mL}$ at $4{ }^{\circ} \mathrm{C}$. PNC and PNE polymers were mixed in a 1:1 mass ratio since they have equal molar ratios of HPMA-Cys and HPMA-ETSA and similar molecular weights. Subsequently, the mixed solution was quickly heated up to $50^{\circ} \mathrm{C}$ using an oil bath and left at $50{ }^{\circ} \mathrm{C}$ for 3 hours. Resulting micellar solutions were dialyzed against water for two days at room temperature and subsequently lyophilized. Micelles were characterized by DLS and NMR both before and after lyophilization.

\section{CryoTEM}

Transmission electron microscopy at cryogenic temperatures (CryoTEM) was done using the TU/e CryoTitan (FEI Company) ${ }^{38}$. Graphene oxide grids, prepared by adding a single layer graphene sheet to a Quantifoil grid $^{39}$, were used for sampling via an automated robot (Vitrobot ${ }^{\mathrm{TM}}$ Mark III, FEI Company), which was kept at room temperature. The excess of liquid sample on the grid was blotted away with filter paper to form a thin film of the 
dispersion. The thin layer of liquid on the grid was plunged rapidly into liquid ethane. The vitrification was done in $100 \%$ humidity atmosphere. A tilt series of 27 cryoTEM images from -65 to +65 degrees with 5 degrees steps were taken to reconstruct the 3D structure of the particles using IMOD, via patch tracking alignment, and AVIZO 9.0 software ${ }^{40}$.

\section{Dynamic light scattering (DLS)}

The size of the micelles was measured by DLS on a Malvern CGS-3 goniometer (Malvern Ltd., Malvern, U.K.), ALV/LSE-5003 Correlator and He-Ne $633 \mathrm{~nm}$ laser. All measurements were carried out at a $90^{\circ}$ angle at temperatures of 10,37 and $45{ }^{\circ} \mathrm{C}$ controlled by a thermostat water bath Julabo FS18. The solvent viscosity was corrected at each temperature by the software. The Z-average radius and polydispersity index was calculated by ALV and DTS software, respectively.

\section{Static light scattering (SLS)}

Weight-average molecular weight of the micelles and the radius of gyration were determined by SLS using an ALV7004 correlator, ALV/LSE-5004 Goniometer, ALV/Dual High QE APD detector unit with fiber splitting device with a set-up of 2 off detection system and a Uniphase Model 1145P He-Ne Laser. The laser wavelength and power were set to 632.8 $\mathrm{nm}$ and $22 \mathrm{~mW}$, respectively and the temperature was controlled by a Julabo CF41 Thermostatic bath.

\section{Ellman assay}

Ellman's reaction was performed on micelles to quantify the crosslink density in the micelles. Cysteine hydrochloride monohydrate standards were prepared at concentrations ranging from 0-1.5 $\mathrm{mM}$ in a 0.1 $\mathrm{M}$ sodium buffer/1 mM EDTA at $\mathrm{pH}$ 8.0. A HiTrap ${ }^{\mathrm{TM}} 1.5 \mathrm{~mL}$, desalting column was equilibrated with the same buffer and was used for separation of micelles from ethyl thioglycolate. A stock solution of $4 \mathrm{mg} / \mathrm{mL}$ of Ellman's reagent was made and $50 \mu \mathrm{L}$ was added to a mixture of $2.5 \mathrm{ml}$ of buffer and $250 \mu \mathrm{L}$ of each standard and ethyl thioglycolate sample. The thiol contents of samples were determined by measuring the absorbance at $\lambda=412 \mathrm{~nm}$ using a BMG spectrostar nano well plate reader.

\section{Preparation of fluorescently labeled micelles}

To fluorescently label the micelles, micelles were formed following the same procedure as described above, then dialyzed and lyophilized. Subsequently, $6 \mathrm{mg}$ of the obtained micelles 
was dissolved in $1 \mathrm{~mL}$ DMSO and $10 \mu \mathrm{L}$ of a $20 \mu \mathrm{g} / \mathrm{mL}$ NHS-Alexa fluor 647 or maleimideAlexa fluor C5 568 stock solution in DMSO was added and left to react overnight. Next, the labeled micelles were dialyzed against DMSO and then water and used for the cellular uptake study.

To make sure enough cysteine moieties were available for conjugation of NHS-Alexa fluor 647, micelles with an excess of cysteine moieties (PNC/PNE ratio of 3:2) were made following the same protocol. Conjugation of maleimide-Alexa fluor C5 568 was conducted on micelles formed from the 1:1 molar ratio of PNC and PNE.

\section{Cellular internalization study}

To investigate the cellular uptake, HeLa and A549 cells were seeded in a glass-bottomed 96 well-plate at a density of $10^{4}$ cells/well and incubated at $37^{\circ} \mathrm{C}$ for $24 \mathrm{~h}$. Then, fluorescently labeled micelles were added and incubated with cells at concentrations of 100, 200, and $400 \mu \mathrm{g} / \mathrm{mL}$ for 2,4 and $24 \mathrm{~h}$ at $37^{\circ} \mathrm{C}$. Subsequently, Hoechst 33430 was added to each well 30 min before imaging with a final concentration of $10 \mathrm{nM}$. The cells were washed twice with PBS and the plate was transferred into a Yokogawa CV7000 (Tokyo, Japan) spinning disk microscope with a 60x 1.2NA water objective.

\section{Cell viability assays}

Cytocompatibility of the formed micelles was assessed by MTS assay ${ }^{41}$. HeLa cells were seeded one day before the experiment into 96-well plates at a density of 8000 cells/well and were maintained in $200 \mu \mathrm{l}$ DMEM Low Glucose $(1 \mathrm{~g} / \mathrm{l})$ medium containing $1 \%$ antibiotics/ antimycotics and $10 \% \mathrm{FBS}$ for $24 \mathrm{~h}$, at $37^{\circ} \mathrm{C}$. A stock solution of the lyophilized micelles at a concentration of $50 \mathrm{mg} / \mathrm{mL}$ in PBS was prepared. The micelle solution was diluted in medium to final concentrations of $1.5,0.75,0.375,0.187,0.093,0.046,0.023$, and $0.011 \mathrm{mg} / \mathrm{mL}$. The cells were incubated with the micelles at different concentrations for 24 $\mathrm{h}$. Then, one washing step was performed using PBS and subsequently, $100 \mu \mathrm{L}$ fresh medium and $20 \mu \mathrm{l}$ MTS reagent was added to the cells and incubated for $2 \mathrm{~h}$. As a negative control group, cells were incubated with $100 \%$ culture medium and as a positive control group, cells were incubated in medium containing $1 \%$ Triton X-100. The cell viability was determined by measuring the absorbance at $492 \mathrm{~nm}$ using a Biochrome EZ microplate reader.

\section{Results and discussion}


Figure 1 shows the overall synthesis scheme of the two complementary polymers, PNC and PNE, containing cysteine and thioester functional groups, respectively. First, the monomer HPMA-ETSA was designed as co-monomer in the thermosensitive blocks of PNE. This monomer was synthesized by conjugation of the hydroxyl group of HPMA to the carboxylic acid functionality of ETSA via a DCC-activated esterification method using DMAP as a catalyst. After column chromatography, the pure monomer was isolated in a yield of $57 \%$ and its structure was confirmed by ${ }^{1} \mathrm{H}-\mathrm{NMR}$ and ${ }^{13} \mathrm{C}-\mathrm{NMR}$ (SI-Fig. 1 ).

PEG with a number-average molecular weight $\left(M_{n}\right)$ of $6 \mathrm{kDa}$ was completely functionalized at both chain ends with bromoisobutyryl bromide groups. In the next step, this PEG macroinitiator was used for the synthesis of PNC and PNE triblock copolymers by ATRP (Fig. $1 \mathrm{~B}$ and $1 \mathrm{C})$.

For PNE, the thermosensitive outer blocks consisted of $N$-isopropylacrylamide (NIPAM) and the above described novel monomer HPMA-ETSA with a ratio of 93:7. The HPMA-ETSA monomer was introduced to obtain a thioester functionality in the thermosensitive domain. After polymerization, the pure polymer was obtained by dialysis and lyophilization in a yield of $81 \%$. The complementary polymer PNC was designed to have a similar structure and size as PNE. Therefore, the same PEG macroinitiator was used for polymerization and a ratio of NIPAM and HPMA-Boc-Cys(Acm) of 93:7 was used. Next, the bromide end groups of PNC polymer chains were substituted by mercaptoethanol to prevent polymer self-crosslinking after deprotection of Boc-cysteine $(\mathrm{Acm})^{42}$.

Several methods have been reported for the polymerization of NIPAM in aqueous solutions ${ }^{43,44}$, here for the first time, we introduced an ATRP method for copolymerization of NIPAM and HPMA-Boc-Cys(Acm) or HPMA-ETSA in aqueous solutions. It has been reported before that the polymerization of HPMA can proceed uncontrolled mainly due to complexation of the amine group to the transition-metal complex ${ }^{45}$. Using ligands with a high complexation constant, such as $\mathrm{Me}_{6}$ TREN and the addition of $\mathrm{CuBr}_{2}$ to increase deactivation rate can improve control over the polymerization ${ }^{45}$. In addition, using solvents with the ability to form hydrogen bonds with polymer and monomers reduces the risk of monomer complexation to catalyst ${ }^{46}$.

Figure $2 \mathrm{~A}$ shows the conversion of monomers during the polymerization of PNE and PNC. For $\mathrm{PNE}$, the residual monomer concentration decreased exponentially over time revealing that the kinetics of polymerization is relatively well-controlled. Moreover, $M_{n}$ evolved linearly with the conversion which indicates that no significant termination due to recombination occurred (Fig. 2C). During the polymerization of PNC, a very fast conversion of HPMA-Boc-Cys(Acm) and NIPAM was observed. The monomer conversion displayed pseudo-first-order kinetics as observed from the linear curves in figure $2 \mathrm{~B}$; however, $\mathrm{Mn}_{\mathrm{n}}$ as a function of conversion did not result in a linear relationship as was observed for PNE (Fig. 
2C). The higher reactivity of HPMA-Boc-Cys(Acm)/ETSA monomers compared to NIPAM's reactivity during polymerization resulted in a not completely random copolymer having a relatively high HPMA-Boc-Cys(Acm)/ETSA content close to the PEG block. The outer parts of polymeric chains in PNC and PNE are mainly formed of PNIPAM only. The PDIs of the obtained polymers (1.7-1.8) are slightly higher than usually observed for ATRP ${ }^{47}$ (Table 1) which can be attributed to the difference in reactivity of acrylamide and methacrylamide monomers, the high length of polymers and the composition of three polymers blocks ${ }^{48}$. A higher $M_{n}$ determined by GPC compared to NMR is often observed for these kinds of polymers and can be explained by the use of PEGs as GPC-standards, which are not perfectly representative polymers to compare hydrodynamic volumes with the synthesized triblock copolymers in the used eluent ${ }^{49,50}$ (Table 1 ).

The cloud point (CP) of PNC prepared by ATRP was $34{ }^{\circ} \mathrm{C}$, which was similar to the $\mathrm{CP}$ of a PNC polymer synthesized by free radical polymerization in a previous study $\left(33^{\circ} \mathrm{C}\right)^{33}$. As expected, the presence of the more hydrophobic monomer HPMA-ETSA in PNE resulted in a slightly lower $\mathrm{CP}\left(29^{\circ} \mathrm{C}\right)$ than the well-known value of $32{ }^{\circ} \mathrm{C}$ for homopolymers of PNIPAM $^{51}$ (Table 1).

Table 1: Characteristics of the two ABA triblock copolymers (PNC and PNE) synthesized by ATRP. Both polymers contain PEG mid blocks of $6 \mathrm{kDa}$ with outer blocks composed of either NIPAM (N) and HPMABoc-Cys(Acm) (C) or NIPAM and HPMA-ETSA (E).

\begin{tabular}{cccccccc}
\hline Polymer & $\begin{array}{c}\text { Feed ratio } \\
{[\mathrm{N}]:[\mathrm{C}] /[\mathrm{E}]}\end{array}$ & $\begin{array}{c}\text { Obtained ratio } \\
{[\mathrm{N}]:[\mathrm{C}] /[\mathrm{E}]^{\mathrm{a}}}\end{array}$ & $\begin{array}{c}\mathrm{M}_{\mathrm{n}}{ }^{\mathrm{a}} \\
(\mathrm{kDa})\end{array}$ & $\begin{array}{c}\mathrm{M}_{\mathrm{n}}{ }^{\mathrm{b}}(\mathrm{kDa}) \\
\mathrm{PDD})^{\mathrm{b}}\end{array}$ & $\begin{array}{c}\mathrm{CP} \\
\left({ }^{\circ} \mathrm{C}\right)\end{array}$ & $\begin{array}{c}\text { Yield } \\
(\%)\end{array}$ \\
\hline PNC & $93: 7$ & $93: 7$ & 42.7 & 83 & 1.82 & $34.1^{\mathrm{c}}$ & 87 \\
PNE & $93: 7$ & $92: 8$ & 42.1 & 67 & 1.72 & 29.2 & 81 \\
\hline
\end{tabular}

a Determined by ${ }^{1} \mathrm{H}-\mathrm{NMR} .{ }^{\mathrm{b}}$ Determined by GPC. ${ }^{\mathrm{c}}$ Cloud point of deprotected PNC 
A

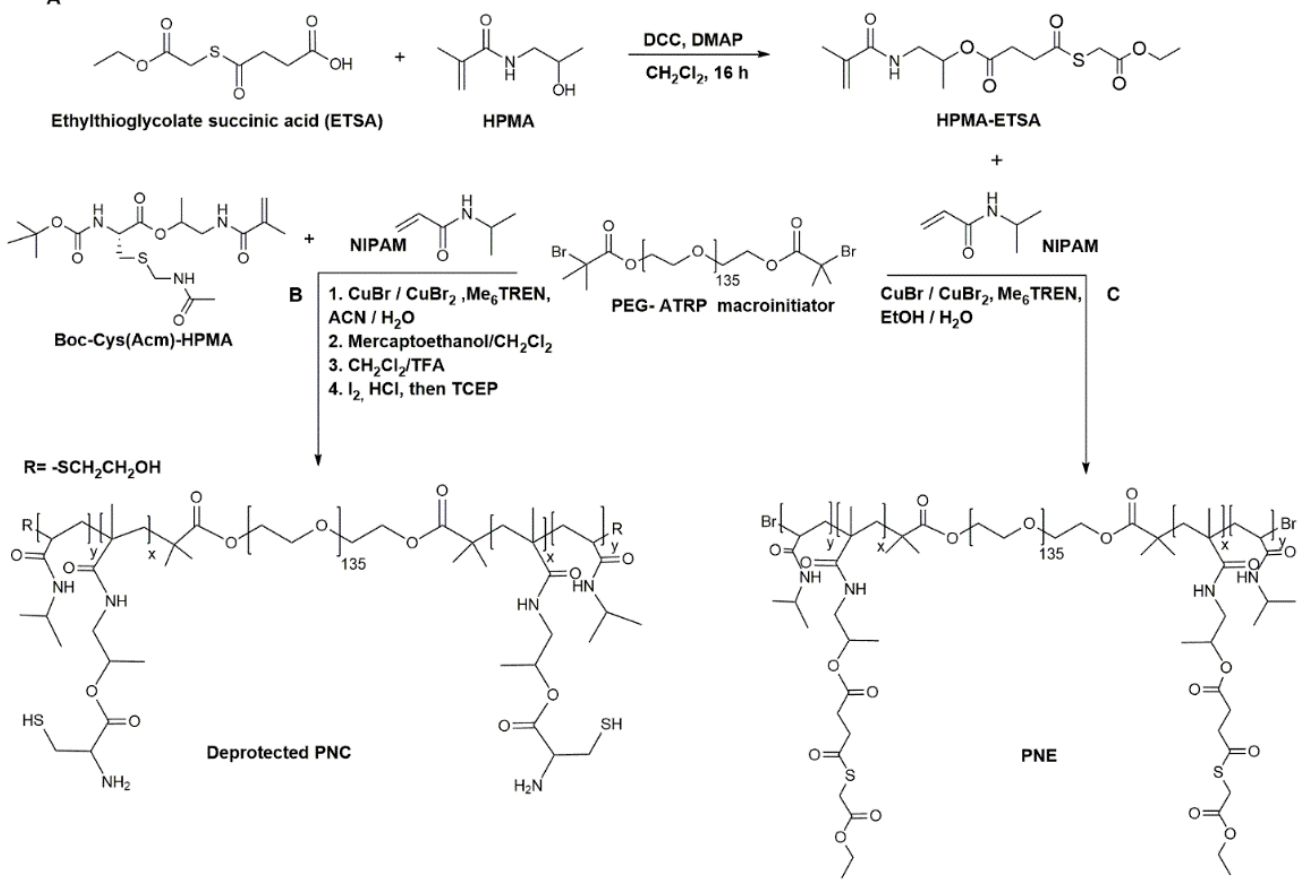

Figure 1: Synthesis route of A) HPMA-ETSA and B-C) ABA tri-block copolymers containing PEG as midblocks and either copolymer of (B) NIPAM and HPMA-Boc-Cys(Acm)(PNC) or (C) NIPAM and HPMAETSA (PNE) as outer-blocks.

A

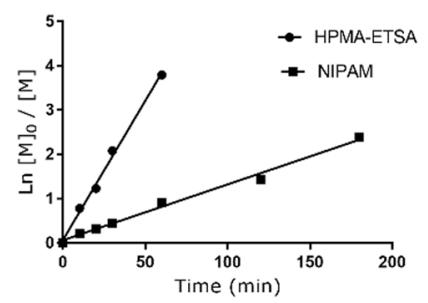

B

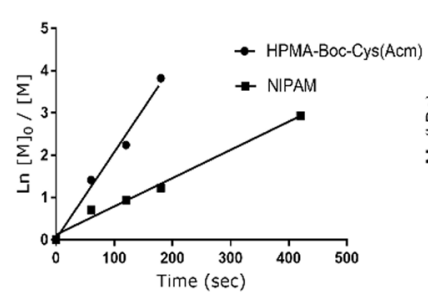

C

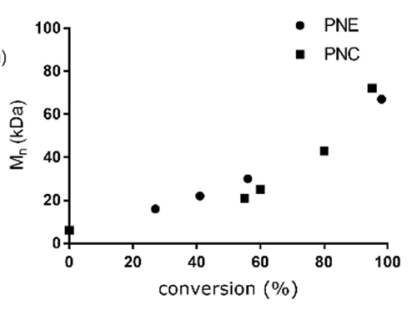

Figure 2: Kinetics of the ATRP of PNE and PNC. A: $\ln \left([M]_{0} /[M]\right)$ as a function of time for the copolymerization of HPMA-ETSA and NIPAM measured by ${ }^{1} \mathrm{H}-\mathrm{NMR}$. B: $\ln \left([\mathrm{M}]_{0} /[\mathrm{M}]\right)$ as a function of time for the copolymerization of HPMA-Boc-Cys(Acm) and NIPAM measured by ${ }^{1} \mathrm{H}-\mathrm{NMR}$. [M] $]_{0}$ : the initial concentration of monomers (NIPAM/HPMA-Boc-Cys(Acm)/HPMA-ETSA) and [M] concentration of monomers in time (NIPAM/HPMA-Boc-Cys(Acm)/HPMA-ETSA). Polymerization of PNC is significantly faster than polymerization of PNE. C: molecular weight evolution of PNC and PNE as a function of monomer conversion. Representative results from one out of three experiments are shown. 


\section{Micelle characterization}

Both PNC and PNE exhibit an increase in solution turbidity above 34.1 and $29.2{ }^{\circ} \mathrm{C}$ respectively, indicating lower critical solution temperature (LCST) behavior. Mixing of these polymers at $4{ }^{\circ} \mathrm{C}$, at a relatively low concentration of $3 \mathrm{mg} / \mathrm{mL}$ followed by fast heating (above the LCST of polymers) resulted in dehydration of the outer A-blocks of the polymers and their self-assembly into flower-like micelles (Fig. 3). The obtained micelles had a diameter of $65 \mathrm{~nm}$ and PDI of 0.11 at $37^{\circ} \mathrm{C}$ (Fig. 4A), similar to previously reported PNIPAMPEG-PNIPAM triblock copolymer micelles ${ }^{3}$. Noteworthy, separate solutions of PNC or PNE displayed formation of similar micelles at high temperature, but micelles disappeared immediately upon cooling. covalent crosslinking of the micellar core by native chemical ligation ${ }^{52}$. To confirm that cross-linking occurred via native chemical ligation (NCL), the formed micelles were passed through a HiTrap ${ }^{\mathrm{TM}}$ desalting column to separate micelles from ethyl thioglycolate, which is the byproduct of the NCL reaction. The Ellman's assay was used to quantify the concentration of released ethyl thioglycolate solution revealing that at least $23 \%$ of HPMA-ETSA monomer contributed to crosslinking. Due to the volatile nature of ethyl thioglycolate (boiling point $=54{ }^{\circ} \mathrm{C}$ ) and therefore partial loss of this compound during the workup the actual percentage of reacted thioester groups is likely much higher. Moreover, the effectiveness of the crosslinking method was examined by lowering the temperature below the LCST of the PNC and PNE polymers to remove the effect of heatinduced micelle formation ${ }^{53}$. By lowering the temperature below the LCST $\left(10^{\circ} \mathrm{C}\right)$, the size of the micelles increased to a diameter of $90 \mathrm{~nm}$ due to rehydration of thermosensitive chains resulting in swelling of the micelles. However, the extent of micelle swelling is limited because of the presence of permanent crosslinks. To investigate the reversibility of this behavior, the temperature was changed between 10 and $37^{\circ} \mathrm{C}$ repeatedly (below and above LCST of PNE and PNC, respectively). As expected, the size of the micelles changed reversibly as a function of temperature, but notably, the PDI was barely affected (Fig. 4B). This "sponge" behavior can be explained by the structure of the polymer as determined from the polymerization kinetics study. According to kinetics study, most HPMA-Cys/ETSA monomers are located next to the PEG chain in both polymers, which results in a crosslinking layer between shell (PEG) and core (PNIPAM segment) of micelles. The PNIPAM segments in the core are relatively flexible and can adjust their conformation depending on temperature resulting in a relatively large difference in size below and above the LCST. The sponge behavior of the micelles is an interesting feature and may be used for loading desirable cargo. 
For storage reasons and to remove the released by-product of NCL, ethyl thioglycolate, the micelles were dialyzed against water and lyophilized. Interestingly, freeze drying, even without a cryo-protectant, hardly affected the micellar size upon resuspension in buffer (Fig. $4 \mathrm{~A})$.

The CryoTEM images confirmed the formation of micelles with uniform spherical shape (Fig. $5 A)$. The size of micelles reported by this method varies from about 50 to $70 \mathrm{~nm}$, which coincides with the data obtained by DLS. The tomographic view reveals that the polymeric micelles have dark patches mostly located in the corona and some inside the micelle together with lighter areas filling the micellar space (Fig. 5B), similar as reported before by Berlepsch et al. for double hydrophobic triblock copolymers ${ }^{54}$. According to an earlier review, the dark patches can be attributed to less hydrated areas in the triblock micelle structure $^{55}$. A close-up of a representative micelle (Fig. 5C) shows the distribution of dark patches in the micelle which is unlike the completely dark micellar core that has been observed in, for example, PEO-PB micelles ${ }^{56}$. The surface representation of an extricated micelle (Fig. $5 \mathrm{C}$ ) shows that these patches are distributed throughout the crosslinked space of the micelle. This can be explained by the fact that during polymer synthesis, most HPMACys/ETSA (crosslinkable monomers) were polymerized close to the PEG block. After micellization and crosslinking, these monomers will be located closer to the micelles' corona and form dense and hydrophobic areas which can be seen as dark patches in tomographic view. The low contrast part in the core of micelles could be assigned as the PNIPAM polymeric chains since they remain hydrated at the temperature of sample preparation (room temperature). These observations are in agreement with the observations of Berlepsch et al. to find patches inside the spherical micelle form instead of a solid single core $^{54}$.

The micelles were studied by ${ }^{1} \mathrm{H}-\mathrm{NMR}$ in $\mathrm{D}_{2} \mathrm{O}$ at 25 and $37^{\circ} \mathrm{C}$, which showed that the signals corresponding to PNIPAM at 1.04 and $3.8 \mathrm{ppm}$ were suppressed by increasing the temperature above the LCST, while the signal corresponding to the protons of PEG at 3.6 ppm remain visible at both temperatures. The disappearance of these characteristic signals confirmed the proposed structure of the micelles with dehydrated PNIPAM hydrophobic cores and hydrated PEG hydrophilic shells (Fig. 6) ${ }^{57}$. 
PNE
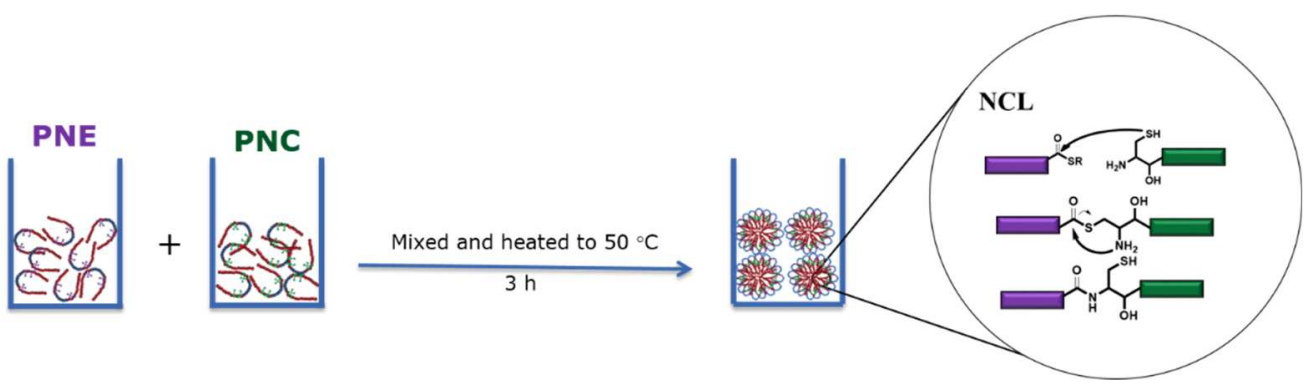

Figure 3: PNC and PNE were dissolved separately in PBS at a concentration of $3 \mathrm{mg} / \mathrm{mL}$ at $4^{\circ} \mathrm{C}$ and afterwards mixed in a 1:1 ratio and immediately heated up to $50{ }^{\circ} \mathrm{C}$ using an oil bath. The micellar solution was left at $50^{\circ} \mathrm{C}$ for $3 \mathrm{~h}$ to let native chemical ligation proceed in the micellar core.

A

B
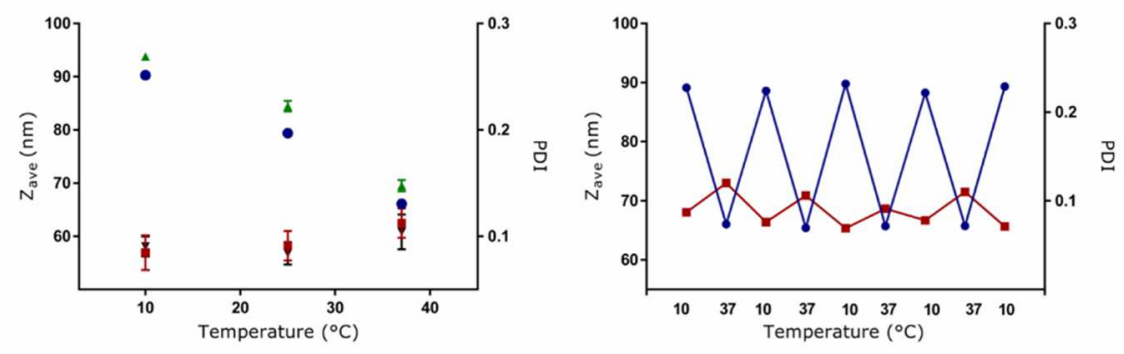

- $z_{\text {ave }}(\mathrm{nm})$

- PDI

A $Z_{\text {ave }}(\mathrm{nm})$ (after lyoph.)

v PDI (after lyoph.)

Figure 4: A) Size of the micelles as a function of temperature, before and after lyophilization. B) Effect of repeated temperature cycles from 10 to $37^{\circ} \mathrm{C}$ on micelle size and PDI.

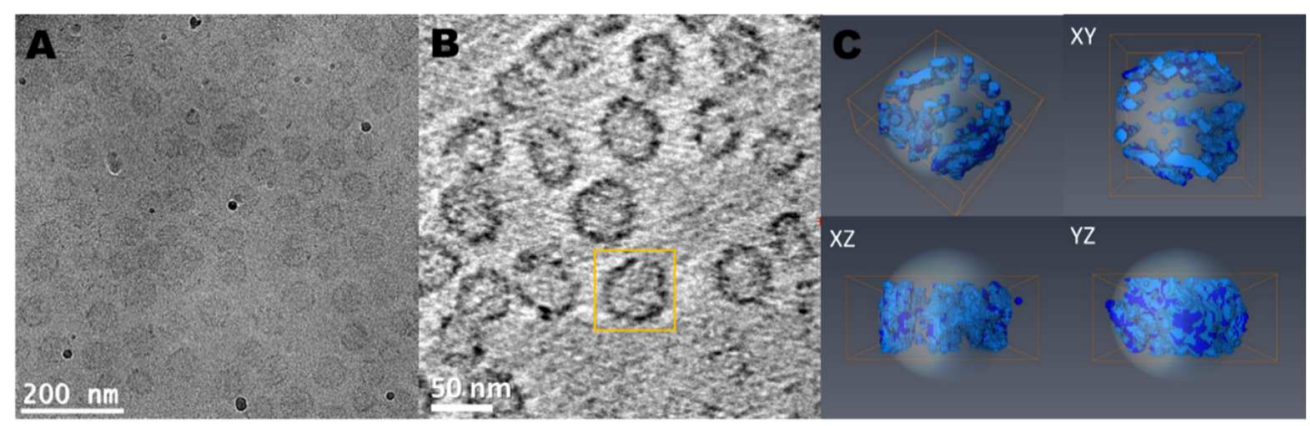

Figure 5: A) CryoTEM images of uniformly sized spherical micelles at a concentration of $3 \mathrm{mg} / \mathrm{mL}$ in water on graphene oxide grid. B) $5 \mathrm{~nm}$ thick tomographic reconstruction of the spherical micelles shown in A. C) A cut-off of the tomographic reconstruction of the selected particle from B in 3D, X-Y, $\mathrm{Y}-\mathrm{Z}$ and $\mathrm{X}-\mathrm{Z}$ views. The bounding box in $\mathrm{C}$ is of $55 \times 55 \times 30 \mathrm{~nm}^{3}$. 


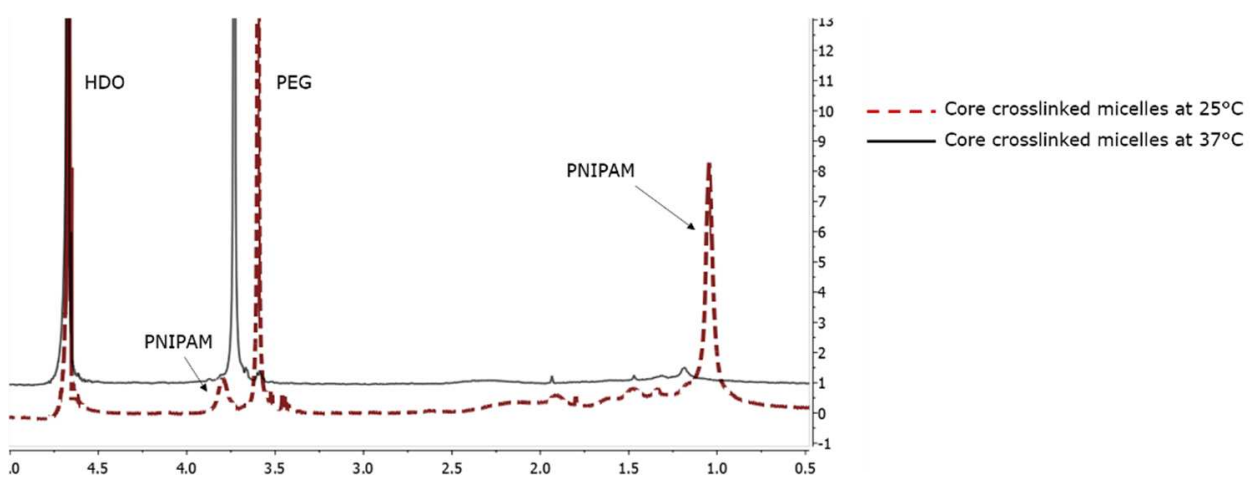

Figure 6: ${ }^{1} \mathrm{H}-\mathrm{NMR}$ spectra of the core crosslinked micelles in $\mathrm{D}_{2} \mathrm{O}$ at 25 and $37^{\circ} \mathrm{C}$. The peak shifts are due to the change in temperature.

The micelles' average molecular weight $\left(\mathrm{M}_{w}\right)$ and aggregation number $\left(N_{a g g}\right)$ was measured at different temperatures of $10,25,37$, and $45^{\circ} \mathrm{C}$ by static light scattering (SLS) (table 2). The obtained data by SLS revealed that the micelles' $N_{a g g}$ and $\mathrm{M}_{w}$ did not change significantly with increasing temperature. Considering the number of PNE polymers in each micelle according to $N_{\text {agg }}$ and crosslink density calculated based on the Ellman's assay, the minimum number of crosslinking points in each micelle is approximately 1000 . This number of crosslinks corroborates with the high stability of the micelles even at low temperatures.

The ratio of the radius of gyration to the hydrodynamic radius $\left(R_{g} / R_{h}\right)$ is an important parameter to understand the conformation of the nanoparticles in solution. $R_{g} / R_{h}$ values of $0.773, \sim 1.8$, and $\sim 2$ have been reported for an uniform sphere, a polydisperse linear coil, and a rod-like linear chain, respectively ${ }^{58,59}$.

Table 2: Characteristics of core crosslinked polymeric micelles consisting of PNE and PNC (1:1) measured by DLS and SLS in PBS.

\begin{tabular}{lllllll}
\hline Temperature & $R_{g}{ }^{a}(\mathrm{~nm})$ & $R_{h}{ }^{b}(\mathrm{~nm})$ & $R_{g} / R_{h}$ & $\begin{array}{l}M_{w \text { (mic.) }} \\
\left(10^{6} \mathrm{Da}\right)\end{array}$ & $\begin{array}{l}\rho_{\text {(mic.) }}{ }^{\mathrm{c}} \\
\left(\mathrm{g} . \mathrm{cm}^{-3}\right)\end{array}$ & $N_{\text {agg }}{ }^{d}$ \\
\hline $10^{\circ} \mathrm{C}$ & 46.3 & 48.2 & 0.95 & 16.37 & 0.06 & 435 \\
$25^{\circ} \mathrm{C}$ & 35.8 & 42.8 & 0.84 & 16.20 & 0.14 & 431 \\
$37^{\circ} \mathrm{C}$ & 29.7 & 35.8 & 0.83 & 14.63 & 0.21 & 389 \\
$45^{\circ} \mathrm{C}$ & 29.9 & 35.0 & 0.85 & 14.24 & 0.20 & 379 \\
\hline
\end{tabular}

${ }^{a}$ Radius of gyration extrapolated to zero concentration. ${ }^{b}$ Hydrodynamic radius extrapolated to zero concentration and zero scattering angle. ${ }^{c}$ Density of the micelles. ${ }^{d}$ Aggregation number of the micelles. 
In this study, the found $R_{g} / R_{h}$ ratios for the micelles $(0.83-0.95)$ were also similar at the various temperatures and close to the theoretical limit for spherical structures. Furthermore, the increase in the density $(\rho)$ of micelles with increasing temperature, clearly shows shrinking of micelles at temperatures above the LCST of the polymers.

\section{Cell study}

To investigate possible biomedical applications of the developed micelles, cellular uptake was studied on HeLa and A549 cells. To this end, two kinds of dyes which also represent model cargos, having maleimide and amine functionalities, were used for labeling the micelles. An NHS modified Alexa fluor 647 was used for conjugation to cysteine moieties while a maleimide-modified Alexa fluor C5 568 was used for conjugation to free thiol groups remaining present after native chemical ligation or of non-reacted cysteine moieties. To conjugate NHS modified dyes, micelles with a molar ratio of 3:2 for PNC/PNE were formed. This ratio ensures that sufficient cysteine groups are present in the core of the micelles, which were used for covalent attachment of dye. For the conjugation of maleimidefunctionalized dye, micelles with 1:1 molar ratio of PNC and PNE were used. To perform the conjugation, the crosslinked micelles were swollen in DMSO, therefore cysteine functionalities or thiol moieties became available for conjugation to the dyes. The swelling of micelles in organic solvent did not result in dissociation due to the presence of covalent crosslinks and no aggregation was observed after extensive dialysis against water to remove excess dye and solvent. The successful covalent conjugation of these two kinds of dyes demonstrated the accessibility of functional groups in the core of micelles for conjugation to desired cargo molecules having different functional groups.

Cells were incubated with labeled micelles for 2, 4, and $24 \mathrm{~h}$, and eventually washed with PBS before imaging. The confocal images in figure 7 showed punctate fluorescence close to the nuclei confirming the internalization of micelles by cells after $24 \mathrm{~h}$ at a concentration of $400 \mu \mathrm{g} / \mathrm{mL}$. At lower concentrations and shorter incubation times, the presence of micelles inside cells was hardly observed, which can be expected for micelles with PEG corona without targeting ligand ${ }^{60}$.

In addition, cytotoxicity of the micelles was studied on HeLa cells. An MTS assay was performed to assess the metabolic activity of cells in the presence of the micelles. In living cells, mitochondrial enzymes reduce the MTS tetrazolium compound and generate a colored formazan that can be quantified by a colorimetric method ${ }^{61}$. Figure 8 shows that no change in mitochondrial activity was observed upon incubation of HeLa cells with micelles at concentrations ranging from $11 \mu \mathrm{g} / \mathrm{mL}$ to $1.5 \mathrm{mg} / \mathrm{mL}$ after $24 \mathrm{~h}$. To study possible cell membrane damage upon exposure to the micelles, an LDH assay was performed. 

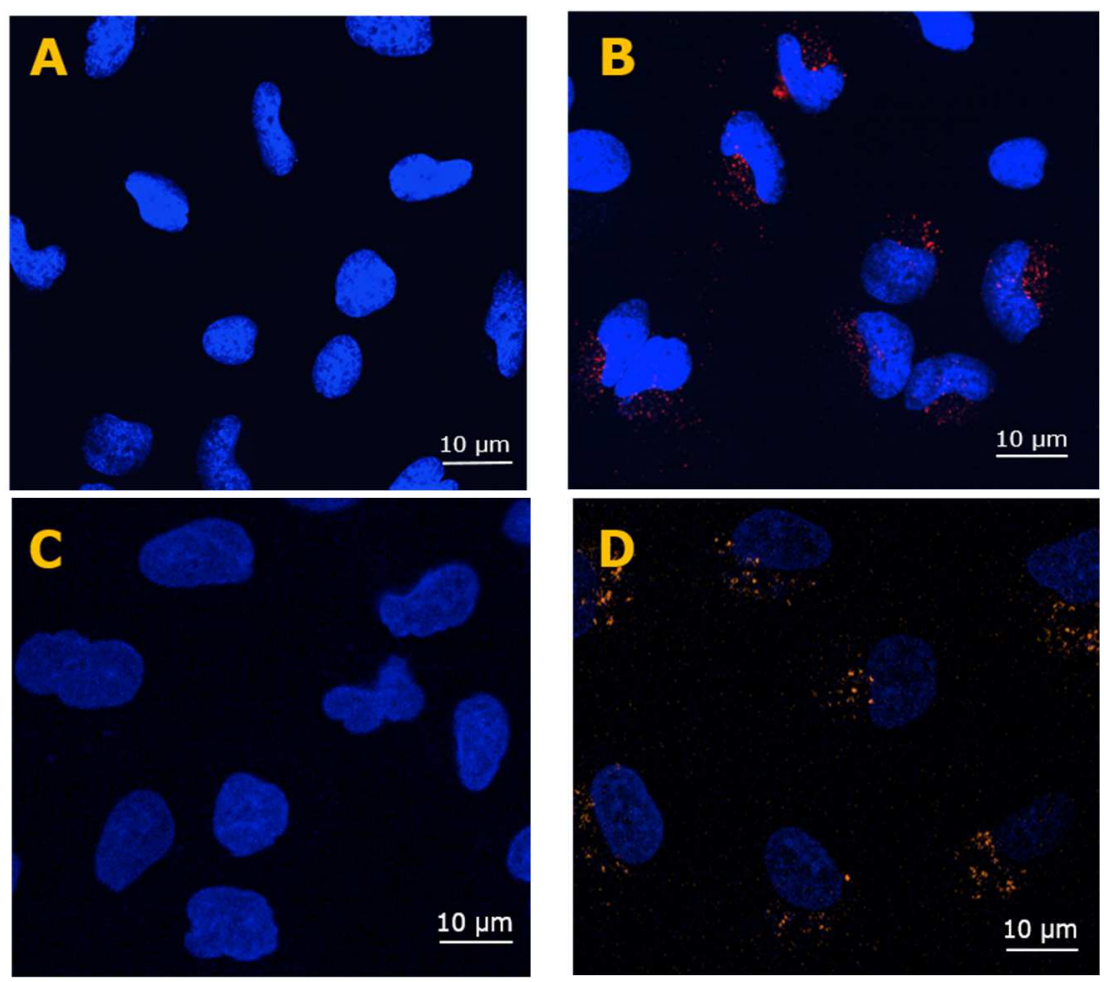

Figure 7: Core crosslinked micelle internalization study. Laser confocal scanning microscopy images of HeLa cells and A549 incubated for $24 \mathrm{~h}$ with (A and C) cell culture medium (B and D) fluorescentlylabeled micelles at a concentration of $400 \mu \mathrm{g} / \mathrm{mL}$. Cell nuclei are stained with Hoechst (blue) while the micelles are visualized by NHS-Alexa fluor 647 in B (red) and maleimide-Alexa flour C5 568 in D (orange).

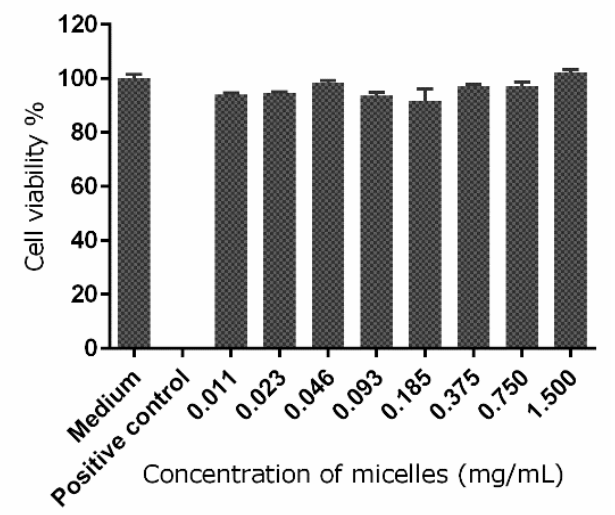

Figure 8: In vitro cytotoxicity (MTS assay) on HeLa cells after $24 \mathrm{~h}$ incubation with micelles across a 135fold concentration range $(0.011-1.5 \mathrm{mg} / \mathrm{mL})$. Data are presented as mean values $\pm S D$ of three independent experiments. 
In damaged cells, lactate dehydrogenase (LDH) is released, which catalyzes a series of reactions that eventually cause reduction of a tetrazolium salt to a highly-colored formazan, which absorbs strongly at $490-520 \mathrm{~nm}^{62}$. The results demonstrated no damage to the cell membrane upon incubation with micelles over a wide range of concentrations (SI-Fig. 5). These results confirm the cytocompatibility of these micelles.

\section{Conclusion}

In this study, native chemical ligation was introduced as a mild, chemoselective method for the crosslinking of micelles in an aqueous medium. Mixing two complementary PNIPAM based ABA polymers, containing either HPMA-Cys or HPMA-ETSA, and subsequently, increasing the temperature above the LCST resulted in flower-like micelles with a size of 65 $\mathrm{nm}$ at $37^{\circ} \mathrm{C}$. Reducing the temperature to $10{ }^{\circ} \mathrm{C}$ resulted in a change in the size of the micelles to $90 \mathrm{~nm}$, but hardly showed any change in polydispersity and aggregation number, thus confirming permanent crosslinking of the micelles. The uniform spherical shape of micelles was confirmed by Cryo-TEM. Interestingly, in tomographic view of the micelles, dark patches were seen close to the corona of micelles, which according to the kinetics study corresponds to the area with the highest content of crosslinkable monomers. Therefore, the dark patches can be interpreted as the crosslinked area of the micelles while the inner low contrast part of micelles is composed of the more hydrated PNIPAM chains. In addition, by adjusting the molar ratio between PNC and PNE polymers during micelle formation, nucleophilic (cysteine) or electrophilic (thioester) sites can be introduced within the micellar core. Here, we have shown that the presence of cysteine and thiol moieties remaining after crosslinking could be used for conjugation of an NHS or maleimide functionalized dyes respectively. These functional sites may also be used for further modification of the micelle to introduce (pro) drugs or charge in the core. Notably, this crosslinking method resulting in the presence of thiol moieties in the micellar core also provides the possibility for conjugation of cargo via a disulfide bond, which may be interesting for intracellular drug delivery. Moreover, the high cell viability and observed cellular uptake of these micelles by HeLa cells show a good cytocompatibility profile and potential of this nanocarrier for drug delivery applications.

\section{Acknowledgments}

The Netherlands Organization for Scientific Research (NWO/VIDI 13457 and NWO/Aspasia 015.009.038) is acknowledged for funding. 


\section{References}

(1) Miyata, K.; Christie, R. J.; Kataoka, K. React. Funct. Polym. 2011, 71 (3), 227-234.

(2) Shi, Y.; van Nostrum, C. F.; Hennink, W. E. ACS Biomater. Sci. Eng. 2015, 1 (6), 393-404.

(3) De Graaf, A. J.; Boere, K. W. M.; Kemmink, J.; Fokkink, R. G.; Van Nostrum, C. F.; Rijkers, D. T. S.; Van Der Gucht, J.; Wienk, H.; Baldus, M.; Mastrobattista, E.; Vermonden, T.; Hennink, W. E. Langmuir 2011, 27, 9843-9848.

(4) Liu, B.; Chen, H.; Li, X.; Zhao, C.; Liu, Y.; Zhu, L.; Deng, H.; Li, J.; Li, G.; Guo, F.; Zhu, X. RSC Adv. 2014, 4 (90), 48943-48951.

(5) Sprakel, J.; Besseling, N. A. M.; Cohen Stuart, M. A.; Leermakers, F. A. M. Eur. Phys. J. E 2008, 25 (2), 163-173.

(6) Oh, K. T.; Oh, Y. T.; Oh, N.; Kim, K.; Lee, D. H.; Lee, E. S. Int. J. Pharm. 2009, 375 (1-2), 163-169.

(7) Lee, E. S.; Oh, K. T.; Kim, D.; Youn, Y. S.; Bae, Y. H. J. Control. Release 2007, 123 (1), 1926.

(8) Lee, W.-C.; Li, Y.-C.; Chu, I.-M. Macromol. Biosci. 2006, 6 (10), 846-854.

(9) Matyjaszewski, K.; Xia, J. Chem. Rev. 2001, 101, 2921-2990.

(10) Jankova, K.; Chen, X.; Kops, J.; Batsberg, W. Macromolecules 1998, 31 (2), 538-541.

(11) Boyer, C.; Corrigan, N. A.; Jung, K.; Nguyen, D.; Nguyen, T.-K.; Adnan, N. N. M.; Oliver, S.; Shanmugam, S.; Yeow, J. Chem. Rev. 2016, 116 (4), 1803-1949.

(12) Gref, R.; Lück, M.; Quellec, P.; Marchand, M.; Dellacherie, E.; Harnisch, S.; Blunk, T.; Müller, R. H. Colloids Surfaces B Biointerfaces 2000, 18 (3-4), 301-313.

(13) Kataoka, K.; Harada, A.; Nagasaki, Y. Adv. Drug Deliv. Rev. 2012, 64 (SUPPL.), 37-48.

(14) Talelli, M.; Rijcken, C. J. F.; Hennink, W. E.; Lammers, T. Curr. Opin. Solid State Mater. Sci. 2012, 16 (6), 302-309.

(15) Talelli, M.; Barz, M.; Rijcken, C. J. F.; Kiessling, F.; Hennink, W. E.; Lammers, T. Nano Today 2015, 10, 93-117.

(16) lijima, M.; Nagasaki, Y.; Okada, T.; Kato, M.; Kataoka, K. Macromolecules 1999, 32 (4), 1140-1146.

(17) Siegwart, D. J.; Whitehead, K. A.; Nuhn, L.; Sahay, G.; Cheng, H.; Jiang, S.; Ma, M.; Lytton-Jean, A.; Vegas, A.; Fenton, P.; Levins, C. G.; Love, K. T.; Lee, H.; Cortez, C.; Collins, S. P.; Li, Y. F.; Jang, J.; Querbes, W.; Zurenko, C.; Novobrantseva, T.; Langer, R.; Anderson, D. G. Proc. Natl. Acad. Sci. 2011, 108 (32), 12996-13001.

(18) Bronich, T. K.; Keifer, P. A.; Shlyakhtenko, L. S.; Kabanov, A. V. J. Am. Chem. Soc. 2005, $127,8236-8237$.

(19) Kim, J. O.; Sahay, G.; Kabanov, A. V.; Bronich, T. K. Biomacromolecules 2010, 11 (4), 919-926. 
(20) Zhang, Z.; Yin, L.; Tu, C.; Song, Z.; Zhang, Y.; Xu, Y.; Tong, R.; Zhou, Q.; Ren, J.; Cheng, J. ACS Macro Lett. 2013, 2 (1), 40-44.

(21) Jiang, X.; Zheng, Y.; Chen, H. H.; Leong, K. W.; Wang, T.-H.; Mao, H.-Q. Adv. Mater. 2010, 22 (23), 2556-2560.

(22) Li, Y.; Xiao, K.; Luo, J.; Xiao, W.; Lee, J. S.; Gonik, A. M.; Kato, J.; Dong, T. A.; Lam, K. S. Biomaterials 2011, 32 (27), 6633-6645.

(23) Fu, R.; Fu, G.-D. Polym. Chem. 2011, 2, 465-475.

(24) Lowe, A. B. Polym. Chem. 2014, 5 (17), 4820-4870.

(25) O’Reilly, R. K.; Joralemon, M. J.; Hawker, C. J.; Wooley, K. L. New J. Chem. 2007, 31 (5), 718-724.

(26) Zhang, J.; Zhou, Y.; Zhu, Z.; Ge, Z.; Liu, S. Macromolecules 2008, 41 (4), 1444-1454.

(27) Xia, Y.; He, H.; Liu, X.; Hu, D.; Yin, L.; Lu, Y.; Xu, W. Polym. Chem. 2016, 7 (41), 63306339.

(28) Bapat, A. P.; Ray, J. G.; Savin, D. A.; Hoff, E. A.; Patton, L.; Sumerlin, B. S. 2012.

(29) Huang, Y.; Sun, R.; Luo, Q.; Wang, Y.; Zhang, K.; Deng, X.; Zhu, W.; Li, X.; Shen, Z. 2016, 99-107.

(30) Johnson, E. C. B.; Kent, S. B. H. J. Am. Chem. Soc. 2006, 128 (20), 6640-6646.

(31) Hu, B.-H.; Su, J.; Messersmith, P. B. Biomacromolecules 2009, 10, 2194-2200.

(32) Boere, K. W. M.; van den Dikkenberg, J.; Gao, Y.; Visser, J.; Hennink, W. E.; Vermonden, T. Biomacromolecules 2015, 16, 2840-2851.

(33) Boere, K. W. M.; Soliman, B. G.; Rijkers, D. T. S.; Hennink, W. E.; Vermonden, T. Macromolecules 2014, 47, 2430-2438.

(34) Zhou, Z.; Chu, B. Macromolecules 1994, 27 (8), 2025-2033.

(35) Temperature-Responsive Polymers: Chemistry, Properties and Applications; V. V. Khutoryanskiy, T. K. G., Ed.; John Wiley \& Sons, Inc., 2018.

(36) Ulbrich, K.; Šubr, V.; Strohalm, J.; Plocová, D.; Jelínková, M.; Říhová, B. J. Control. Release 2000, 64 (1-3), 63-79.

(37) Loccufier, J.; Van Bos, M.; Schacht, E. Polym. Bull. 1991, 27 (2), 201-204.

(38) Patterson, J. P.; Xu, Y.; Moradi, M.-A.; Sommerdijk, N. A. J. M.; Friedrich, H. Acc. Chem. Res. 2017, 50 (7), 1495-1501.

(39) van de Put, M. W. P.; Patterson, J. P.; Bomans, P. H. H.; Wilson, N. R.; Friedrich, H.; van Benthem, R. A. T. M.; de With, G.; O’Reilly, R. K.; Sommerdijk, N. A. J. M. Soft Matter 2015, 11 (7), 1265-1270.

(40) Wirix, M. J. M.; Bomans, P. H. H.; Hendrix, M. M. R. M.; Friedrich, H.; Sommerdijk, N. A. J. M.; de With, G. J. Mater. Chem. A 2015, 3 (9), 5031-5040.

(41) Malich, G.; Markovic, B.; Winder, C. Toxicology 1997, 124 (3), 179-192.

(42) Tsarevsky, N. V.; Matyjaszewski, K. Macromolecules 2002, 35 (24), 9009-9014. 
(43) de Graaf, A. J.; Mastrobattista, E.; Vermonden, T.; van Nostrum, C. F.; Rijkers, D. T. S.; Liskamp, R. M. J.; Hennink, W. E. Macromolecules 2012, 45 (2), 842-851.

(44) Zhang, Q.; Wilson, P.; Li, Z.; McHale, R.; Godfrey, J.; Anastasaki, A.; Waldron, C.; Haddleton, D. M. J. Am. Chem. Soc. 2013, 135 (19), 7355-7363.

(45) Teodorescu, M.; Matyjaszewski, K. Macromolecules 1999, 32 (15), 4826-4831.

(46) Xia, Y.; Yin, X.; Burke, N. a D.; Sto, H. D. H. Macromolecules 2005, 5937-5943.

(47) Masci, G.; Giacomelli, L.; Crescenzi, V. Macromol. Rapid Commun. 2004, 25, 559-564.

(48) Handbook of Radical Polymerization; Matyjaszewski, K., Davis, T. P., Eds.; John Wiley \& Sons, Inc.: Hoboken, NJ, USA, 2002.

(49) Smithenry, D. W.; Kang, M.-S.; Gupta, V. K. Macromolecules 2001, 34 (24), 8503-8511.

(50) Su, W.-F. Principles of Polymer Design and Synthesis; Lecture Notes in Chemistry; Springer Berlin Heidelberg: Berlin, Heidelberg, 2013; Vol. 82.

(51) Schild, H. G. Prog. Polym. Sci. 1992, 17, 163-249.

(52) Dawson, P. E.; Kent, S. B. H. Annu. Rev. Biochem. 2000, 69 (1), 923-960.

(53) Wei, H.; Cheng, S.-X.; Zhang, X.-Z.; Zhuo, R.-X. Prog. Polym. Sci. 2009, 34, 893-910.

(54) Berlepsch, H. v.; Böttcher, C.; Skrabania, K.; Laschewsky, A. Chem. Commun. 2009, No. $17,2290$.

(55) Holder, S. J.; Sommerdijk, N. A. J. M. Polym. Chem. 2011, 2 (5), 1018.

(56) Zheng, Y.; Won, Y.-Y.; Bates, F. S.; Davis, H. T.; Scriven, L. E.; Talmon, Y. J. Phys. Chem. B 1999, 103 (47), 10331-10334.

(57) Soga, O.; van Nostrum, C. F.; Ramzi, A.; Visser, T.; Soulimani, F.; Frederik, P. M.; Bomans, P. H. H.; Hennink, W. E. Langmuir 2004, 20 (21), 9388-9395.

(58) Aharoni, S. M; Murthy, N. S. Polym. Commun. 1983, 24 (5), 132-136.

(59) Van de Sande, W.; Persoons, A. J. Phys. Chem. 1985, 89 (3), 404-406.

(60) Mishra, S.; Webster, P.; Davis, M. E. Eur. J. Cell Biol. 2004, 83 (3), 97-111.

(61) Berridge, M. V.; Herst, P. M.; Tan, A. S. In Biotechnology Annual Review; 2005; pp 127152.

(62) Khattak, S. F.; Spatara, M.; Roberts, L.; Roberts, S. C. Biotechnol. Lett. 2006, 28 (17), 1361-1370. 


\section{Supporting information}

A

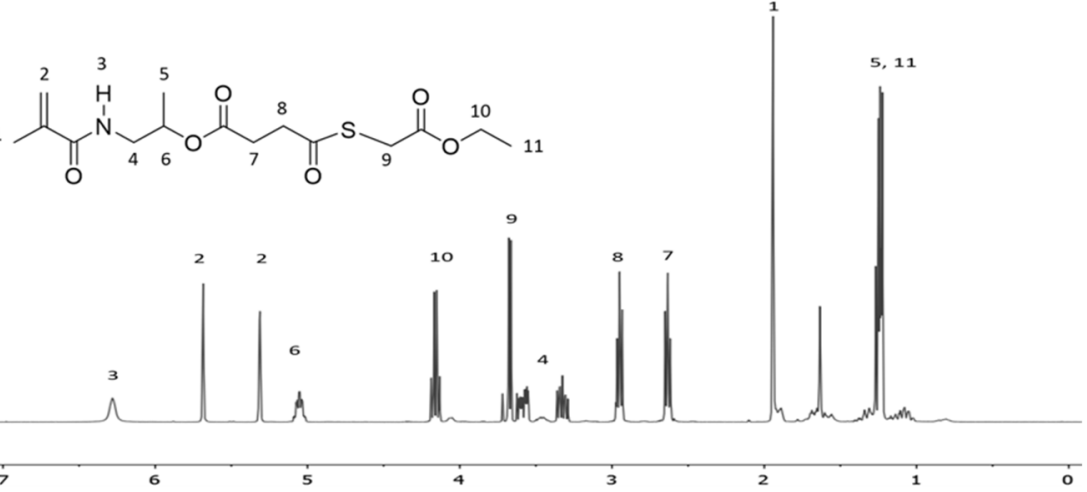

B

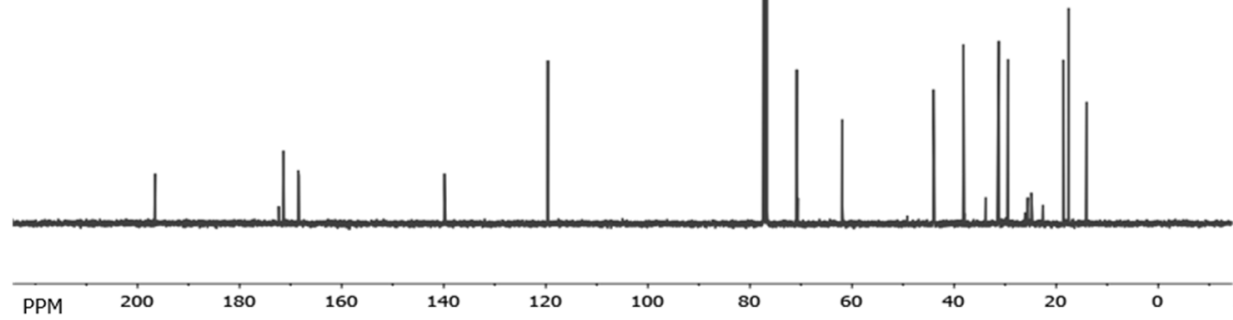

SI-Figure 1: A) ${ }^{1} \mathrm{H}-\mathrm{NMR}$ spectrum of HPMA-ETSA in $\left.\mathrm{CDCl}_{3} \mathrm{~B}\right){ }^{13} \mathrm{C} \mathrm{NMR}^{\mathrm{N}}$ spectrum of HPMA-ETSA in $\mathrm{CDCl}_{3}$.

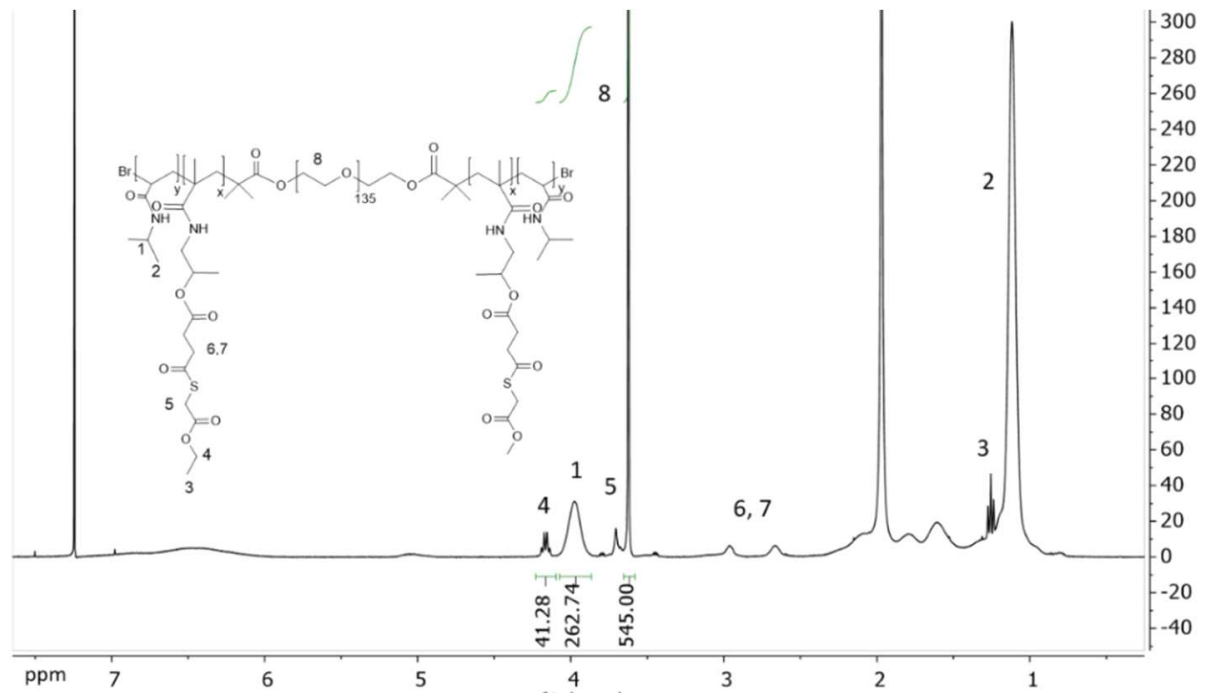

SI-Figure 2: ${ }^{1} \mathrm{H}-\mathrm{NMR}$ spectrum of PNE in $\mathrm{CDCl}_{3}$. 


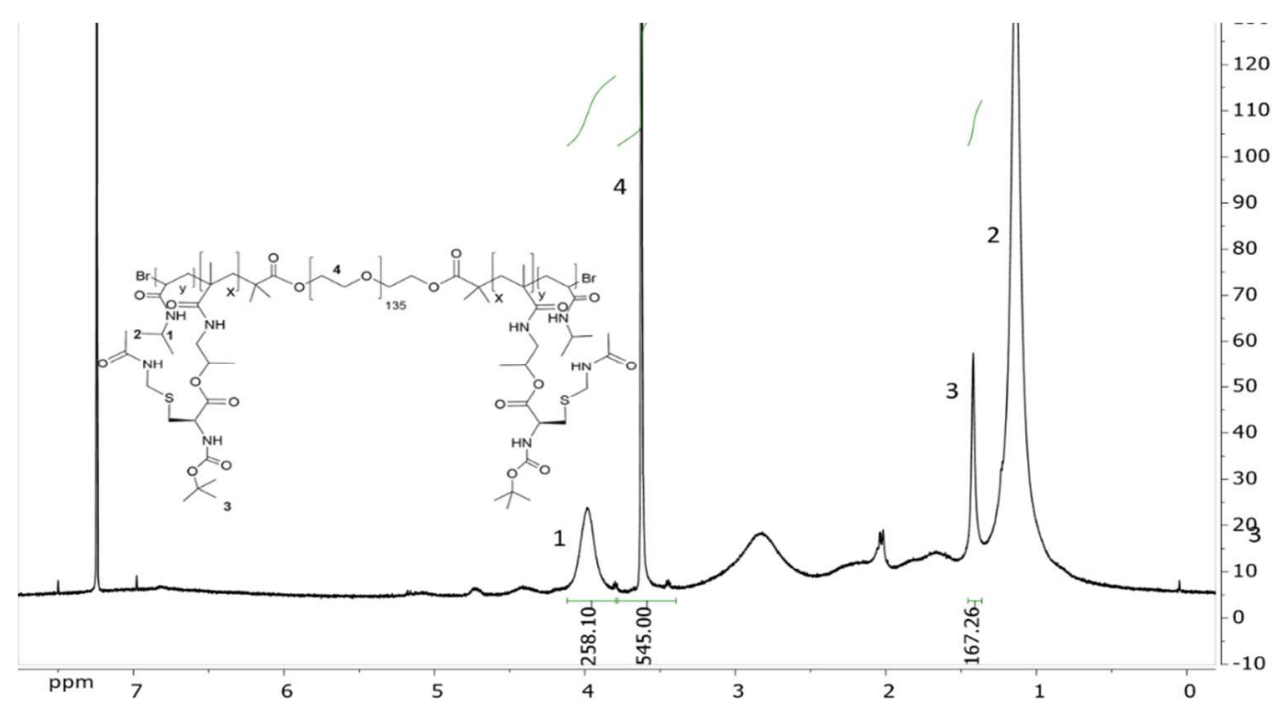

SI-Figure 3: ${ }^{1} \mathrm{H}-\mathrm{NMR}$ spectrum of $\mathrm{PNC}$ in $\mathrm{CDCl}_{3}$.

A

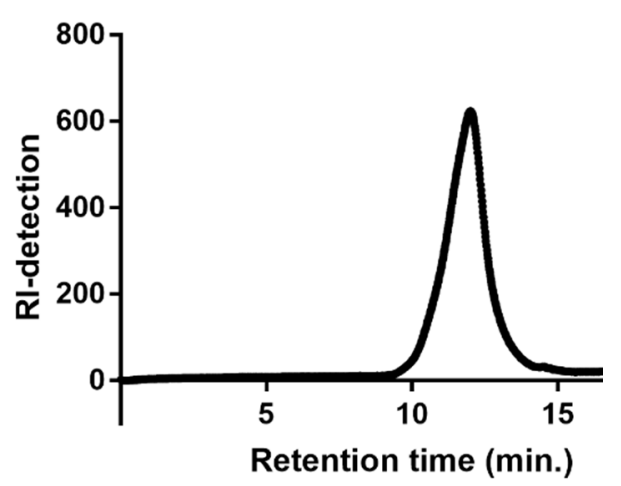

B

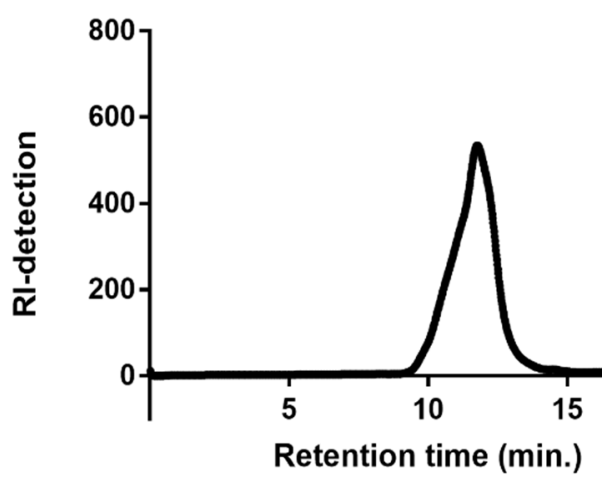

SI-Figure 4: A) GPC chromatogram of PNE, B) GPC chromatogram of PNC in DMF contaning $10 \mathrm{mM} \mathrm{LiCl.}$ 


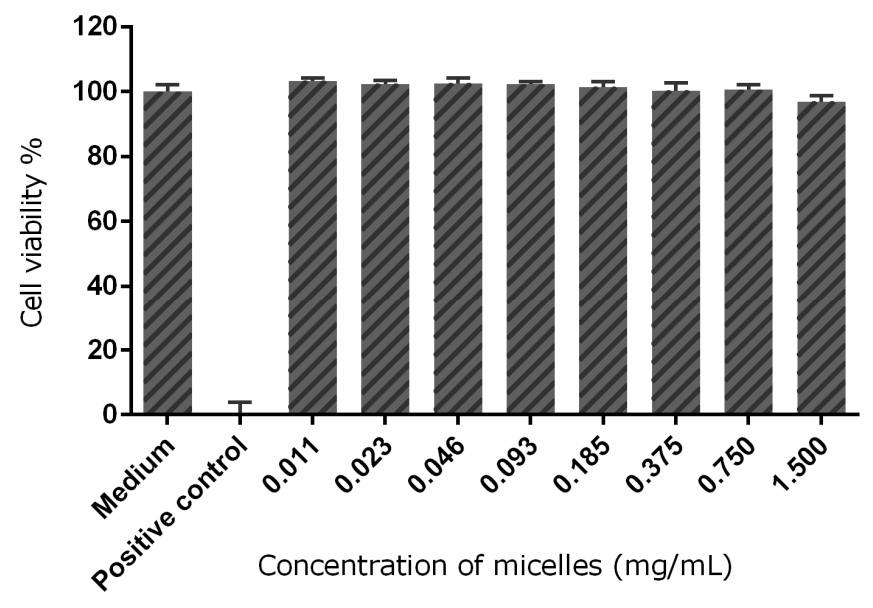

SI-Figure 5: LDH assay on HeLa cells at concentrations ranging from $0.011-1.5 \mathrm{mg} / \mathrm{mL}$. 


\section{Chapter 4}

\section{The influence of PEG conformation in flower- and star-like micelles on circulation time and accelerated blood clearance}

M. Najafi ${ }^{1}$, M. H. A. M. Fens ${ }^{1}$, C. A. de Jongh ${ }^{1,2}$, P. Boross ${ }^{2}$, I. K. Ventouri' ${ }^{3}$, K. Dijkxhoorn², L. A. L. Fliervoet ${ }^{1}$, A. A. Varela Moreira ${ }^{1,4}$, R. Fokkink ${ }^{5}$, A. Astefanei ${ }^{3}$, P. J. Schoenmakers ${ }^{3}$, C. F. van Nostrum ${ }^{1}$, W. E. Hennink ${ }^{1}$, T. Vermonden ${ }^{1}$

${ }^{1}$ Department of Pharmaceutics, Utrecht Institute for Pharmaceutical Sciences (UIPS), Science for Life, Faculty of Science, Utrecht University, P.O. Box 80082, 3508 TB Utrecht, the Netherlands ${ }^{2}$ Center for Translational Immunology, University Medical Center Utrecht, P.O. Box 85500, 3508 GA, Utrecht, the Netherlands

${ }^{3}$ Analytical Chemistry Group, van't Hoff Institute for Molecular Sciences, University of Amsterdam, Science Park 904, 1098 XH Amsterdam, the Netherlands

${ }^{4}$ Department of Clinical Chemistry and Haematology, University Medical Center Utrecht, P.O. Box 85500, 3508 GA, Utrecht, the Netherlands

${ }^{5}$ Physical Chemistry and Soft Matter, Wageningen University \& Research, Stippeneng 4, 6708 WE Wageningen, the Netherlands 


\section{Abstract}

In this study, we investigated the effect of PEG conformation on circulation kinetics and accelerated blood clearance ( $A B C$ phenomenon) of flower- and star-like micelles after intravenous administration. To this end, two types of $A B A$ and two types of $A B$ block copolymers were synthesized by atom transfer radical polymerization (ATRP) using poly(ethylene glycol) (PEG) as a macroinitiator (B block). The A blocks were composed of $\mathrm{N}$ isopropylacrylamide (NIPAM; 92 mol\%) and $\sim 8$ mol\% of either $\mathrm{N}$-(2-hydroxypropyl) methacrylamide-cysteine (HPMA-Cys) or N-(2-hydroxypropyl)methacrylamideethylthioglycolate succinic acid (HPMA-ETSA). In aqueous solution and at a temperature (50 ${ }^{\circ} \mathrm{C}$ ) above the cloud point of the A blocks, the ABAs ( $\left.\sim 0 \mathrm{kDa}\right)$ and $\mathrm{ABs}(\sim 20 \mathrm{kDa})$ copolymers self-assembled into flower-like and star-like micelles, respectively. Both types of micelles had a PEG shell with a loop conformation in flower-like and a brush conformation (and with a methoxy end group) in star-like micelles and a PNIPAM based core. The micellar core was covalently crosslinked by native chemical ligation between thioester functionalized monomers (HPMA-ETSA) and cysteines in HPMA-Cys monomers present in the thermosensitive blocks. Dynamic light scattering showed a Z-average diameter of $65 \pm 2$ (PDI 0.1 ) and $83 \pm 2 \mathrm{~nm}$ (PDI 0.05) for flower-, and star-like micelles, respectively. Static light scattering analysis showed aggregation numbers of $\sim 400$ and $\sim 1700$ for flower- and starlike micelles, respectively, meaning a surface area per PEG chains of $\sim 37$ versus $12 \mathrm{~nm}^{2}$. The circulation kinetics of these micelles in immunocompetent mice after a single intravenous (i.v.) injection were studied and it was revealed that flower-like micelles have a significantly longer circulation half-life compared to star-like micelles (18.6 \pm 3.1 versus $10.7 \pm 0.7 \mathrm{~h}$ ). To investigate the possible occurrence of accelerated blood clearance $(A B C)$ phenomenon, each of the above groups was divided into two subgroups receiving a second i.v. injection of either flower- or star-like micelles, seven days after the first injection when star-like micelles were injected twice, accelerated blood clearance was observed. Importantly, after injection of flower-like micelles, whether applied as a first, second of both injections, no $A B C$ effect was seen. Production of anti-PEG IgM upon injection of both types of micelles was proved by ELISA. Moreover, ELISA revealed binding of anti-PEG antibodies (IgM and IgG) to star-like micelles but not to flower-like micelles.

Taken together, the observed difference in circulation times and binding of anti-PEG antibodies to star- and flower-like micelles is likely related to the difference in PEG conformation. According to previous reports, the methoxy end group of PEG triggers immune system resulting in the formation of anti-PEG antibodies. Therefore, introducing PEG in a looped rather than a brush conformation on nanoparticles is an attractive and so 
The influence of PEG conformation in flower- and star-like micelles on circulation time and accelerated blood clearance

far unexploited and unexplored option to prolong circulation time and to avoid the occurrence of the unwanted $A B C$ phenomenon upon repeated injections of pegylated nanomedicines. 


\section{Introduction}

Polymeric micelles are formed from amphiphilic block copolymers in aqueous solutions and have been studied extensively to facilitate the (targeted) delivery of mainly hydrophobic drugs to specific tissues ${ }^{1-4}$. Poly(ethylene glycol) (PEG) has been widely applied as hydrophilic block to prolong the systemic circulation of micelles due to its so-called "stealth" properties ${ }^{5-7}$. This is ascribed to PEG's specific physicochemical characteristics including high water solubility and high chain mobility, which result in the formation of a hydration zone around the surface of nanoparticles. The presence of this hydrated zone limits adsorption of opsonins and consequently decreases recognition of PEGylated nanoparticles by cells of the mononuclear phagocyte system (MPS) ${ }^{8}$. However, pegylated nanoparticles are not inert and do not fully suppress protein adsorption referred to as the protein corona ${ }^{7,9-11}$. For instance, it was shown that PEG coated nanoparticles have the tendency to form a protein corona that contains an abundance of clusterin proteins. This corona reduces unspecific uptake of these particles by macrophages ${ }^{9}$. Despite the increased circulation time and excellent colloidal stability of PEGylated nanoparticles compared to non-PEGylated counterparts both in vitro and in vivo ${ }^{12-15}$ there are publications in which the ability of PEG to induce the production of anti-PEG antibodies mainly of the IgM isotype was demonstrated $^{16,17}$. These anti-PEG IgMs are considered to be responsible for the rapid clearance of PEGylated therapeutics upon repeated administration, which is known as the accelerated blood clearance $(A B C)$ phenomenon ${ }^{18-21}$. It is hypothesized that the first administration of PEGylated therapeutics triggers the production of anti-PEG IgM by splenic marginal zone $B$ cells ${ }^{19}$. Upon subsequent administrations of PEGylated therapeutics, antiPEG IgM recognizes and binds to the particles leading to opsonization by C3 fragments and ultimately enhanced uptake by phagocytes such as Kupffer cells via complement receptormediated endocytosis ${ }^{22}$. Several parameters have been described that may affect the extent of anti-PEG antibody production, such as particle size ${ }^{23}$, injected dose, PEG molecular weight and density ${ }^{24}$, and the nature of the terminal end group on $\mathrm{PEG}^{25}$. For instance, methoxy $\left(\mathrm{OCH}_{3}\right)$ end groups trigger the production of higher titers of anti-PEG IgM than hydroxy $(\mathrm{OH})$, carboxyl $(\mathrm{COOH})$ and amino $\left(\mathrm{NH}_{2}\right)$ end groups in PEGylated nanoparticles ${ }^{26}$. However, for all pegylated nanoparticles with different terminal PEG groups, significant clearance of a second dose was reported as a consequence of opsonization by C3 fragments and complement activation ${ }^{26}$. The $A B C$ phenomenon occurs in animals e.g. mice ${ }^{27}$, rats $^{18}$, beagle dogs ${ }^{28}$, cynomolgus monkeys, and mini pigs ${ }^{29}$. Moreover, in a number of publications it has been demonstrated that the induction of anti-PEG antibodies also occurs in humans treated with PEGylated therapeutics or those persistently exposed to PEG-containing 
products $^{17,30-33}$. Armstrong et al. showed that the presence of anti-PEG antibodies in plasma was strongly associated with rapid clearance of PEG-asparaginase in patients treated for acute lymphoblastic leukemia ( $A L L)^{34}$. Therefore, the $A B C$ phenomenon is an important clinical concern for PEGylated proteins, nanoparticles and nanomedicines and logically finding a strategy to circumvent PEG-induced immunogenicity is of high interest ${ }^{22}$. Besides the factors mentioned above, the PEG density on the surface has also shown to affect the circulation kinetics of PEGylated nanoparticles ${ }^{15,35,36}$. In these papers it was shown that with an increasing PEG density, the PEG chains lose conformational freedom, resulting in an enhanced barrier for proteins to diffuse in the shell of hydrated PEG chains which in turn results in reduction of protein adsorption on the surface of PEGylated nanoparticles ${ }^{37,38}$. Interestingly, the PEG chain flexibility varies depending on the PEG conformation on the micelles' corona. For instance, de Graaf et al. compared the flexibility of PEG chains in starand flower-like micelles and demonstrated using NMR analysis that the PEG chains in the corona of star- and flower-like micelles are attached to the micellar core through one and two rigid segments, respectively. NMR analysis showed that the number of rigid ethylene oxide units in flower-like micelles is around two times higher than that of star-like micelles (38 and 16 units for PEG 6 and 2 kDa for flower- and star-like micelles, respectively). Moreover, the flexible segments shown to have a lower mobility in the flower-like micelles compared to star-like micelles ${ }^{39}$. Thus, PEG chains in flower-like micelles are forced into a more mushroom conformation than the brush-like conformation in star-like micelles limiting their flexibility ${ }^{39}$. Importantly, the effect of PEG conformation on the circulation kinetics of these types of micelles has not been studied yet.

In this study, we therefore aim to investigate the effect of the PEG conformation on the physical properties e.g. size, $\zeta$-potential, aggregation number $\left(\mathrm{N}_{\mathrm{agg}}\right)$ of micelles, as well as circulation kinetics upon single and second intravenous injection in immunocompetent mice. To this end, two complementary $A B A$ and two complementary $A B$ block copolymers consisting of PEG as hydrophilic B-block and a thermosensitive copolymers of either $N$ isopropylacrylamide (NIPAM) and N-(2-hydroxypropyl)methacrylamide-cysteine (HPMACys), P(NIPAM-co-HPMA-Cys), or NIPAM and N-(2-hydroxypropyl)methacrylamideethylthioglycolate succinic acid (HPMA-ETSA), P(NIPAM-co-HPMA-ETSA) as A-block(s) were synthesized. Flower- and star-like micelles were prepared based on these $A B A$ or $A B$ thermosensitive block copolymers and core crosslinked by native chemical ligation as reported before ${ }^{40}$. Sizes and aggregation numbers of the two types of micelles were determined using dynamic and static light scattering (DLS and SLS). The circulation kinetics and biodistribution upon intravenous injection of these micelles were investigated in immunocompetent BALB/c mice. Importantly, the in vivo behavior of both types of micelles 
upon a second injection was studied to assess the possible occurrence of accelerated blood clearance $(A B C)$.

\section{Materials and Methods}

\subsection{Materials}

All commercial chemicals were obtained from Sigma-Aldrich (Zwijndrecht, the Netherlands) and used as received unless indicated otherwise. N-(2-hydroxypropyl)methacrylamide (HPMA) was synthesized by a reaction of methacryloyl chloride with 1-aminopropan-2-ol in dichloromethane according to a literature procedure ${ }^{41}$. Peptide grade dichloromethane $\left(\mathrm{CH}_{2} \mathrm{Cl}_{2}\right)$ was obtained from Biosolve (Valkenswaard, the Netherlands). N-(2-hydroxypropyl) methacrylamide-Boc-S- acetamidomethyl-L-cysteine (HPMA-Boc-Cys-(Acm)) and N-(2hydroxypropyl) methacrylamid-ethylthioglycolate succinic acid (HPMA-ETSA) were synthesized as previously reported ${ }^{40,42}$. Poly(ethylene glycol) bis(2-bromoisobutyrate) and methoxypolyethylene glycol 2-bromoisobutyrate were synthesized according to a previously reported method ${ }^{39}$, 40 . Phosphate buffered saline $10 X(P B S)$ pH $7.4(1.37 \mathrm{M} \mathrm{NaCl}$, $0.027 \mathrm{M} \mathrm{KCl}$ and $0.119 \mathrm{M}$ phosphates) BioReagents were purchased from B. Braun (Melsungen, Germany), which was diluted (1:10) to yield a final composition of $(0.13 \mathrm{M}$ $\mathrm{NaCl}, 2.7 \mathrm{mM} \mathrm{KCl}$ and $11.9 \mathrm{mM}$ phosphates, $\mathrm{pH} 7.4$ ) and used in all experiments. Alexa Fluor $^{\mathrm{TM}} 750$ was obtained from Thermo Fisher Scientific (Massachusetts, United States). PD10 desalting columns were purchased from GE Healthcare (Uppsala, Sweden). PEG standards (molecular weights ranging from 106 to $969000 \mathrm{Da}$ ) for GPC characterization were purchased from Agilent Technologies BV (Santa Clara, US). Dialysis tubes (molecular weight cut-off (MWCO) 3.5-10 kDa) were obtained from Fisher Scientific (Bleiswijk, the Netherlands). Mice (BALB/c) were obtained from Envigo, the Netherlands. PEGylated granulocyte colony-stimulating factor (PEG-G-CSF) (PEGfilgrastim/Neulasta) was purchased from Amgen (California, USA). Horseradish peroxidase (HRP)-conjugated anti-mouse IgG and biotinylated anti-mouse IgM were obtained from Southern Biotech (Birmingham, USA). Streptavidin-horseradish peroxidase (SA-HRP) was purchased from Roche (Basel, Switzerland). BM blue (3,3',5,5'-tetramethylbenzidine (TMB) was obtained from Invitrogen (California, USA). Anti-PEG IgM (ab133471) specific to PEG backbone and IgG specific to methoxy group (ab190652) were purchased from Abcam (Cambridge, UK). HRP-conjugated swine anti-rabbit Ig was purchased from Dako Agilent, (Santa Clara, USA). Human sera were obtained from University Medical Center Utrecht minidonor service (donors ID: 2019725 and 2017183) and IgM and IgG titers were determined by ELISA. 


\subsection{Synthesis of P(NIPAM-co-HPMA-Cys)-PEG-P(NIPAM-co-HPMA-Cys), PNC and m-PEG-P(NIPAM-co-HPMA-Cys), m-PNC}

ATRP macroinitiators of poly(ethylene glycol) bis(2-bromoisobutyrate $)^{40}(50 \mathrm{mg}, 7.9 \mu \mathrm{mol})$ and methoxy polyethylene glycol 2-bromoisobutyrate ${ }^{39}(50 \mathrm{mg}, 16 \mu \mathrm{mol})$ were used for the preparation of PNC and $\mathrm{mPNC}$, respectively. In addition to the PEG-macroinitiator, $\mathrm{CuBr}$ (4.5 $\mathrm{mg}, 0.031 \mathrm{mmol}), \mathrm{CuBr}_{2}(4.7 \mathrm{mg}, 0.021 \mathrm{mmol}), \operatorname{NIPAM}(268.9 \mathrm{mg} ; 2.38 \mathrm{mmol}$ ) and HPMABoc-Cys-(Acm) $(67 \mathrm{mg}, 0.16 \mathrm{mmol})$ were dissolved in a mixture of $2.8 \mathrm{~mL}$ of water and 0.9 $\mathrm{mL}$ acetonitrile. The mixtures were stirred and deoxygenated by flushing with nitrogen for $15 \mathrm{~min}$. at room temperature, followed by $15 \mathrm{~min}$. flushing in an ice bath. After addition of $16 \mu \mathrm{L}\left(0.06 \mathrm{mmol}\right.$ ) of tris[2-(dimethylamino)ethyl]amine (Me $6{ }_{6}$ TREN) to the polymerization flasks, polymerization started, and the color of the mixture changed to blue. The reaction mixtures were stirred for $2 \mathrm{~h}$ in an ice bath and subsequently the crude products were diluted with water to $15 \mathrm{~mL}$ and dialyzed (MWCO, $10 \mathrm{kDa}$ ) against water at room temperature for two days and obtained in solid form after lyophilization. In the next step, the acetamidomethyl (Acm) and tert-butyloxycarbonyl (Boc) protecting groups of cysteines were removed as described before ${ }^{43}$. Briefly, Boc protecting groups of cysteines were removed by dissolving $250 \mathrm{mg}$ of the protected PNC (or mPNC) in a mixture of dry DCM and trifluoroacetic acid (TFA) $(1: 1 \mathrm{v} / \mathrm{v}, 10 \mathrm{~mL})$. These mixtures were stirred for $1 \mathrm{~h}$ at room temperature, the solvent was subsequently evaporated under reduced pressure and the crude products were dissolved in $2 \mathrm{~mL} D C M$ and precipitated in an excess of cold diethyl ether. Subsequently, the Acm protecting groups of cysteines were removed by dissolving the polymers $(250 \mathrm{mg})$ in a mixture of methanol $(\mathrm{MeOH})$ and water $(1: 1 \mathrm{v} / \mathrm{v}, 10 \mathrm{~mL})$ under a nitrogen atmosphere. Next, $500 \mu \mathrm{L} \mathrm{HCl}(1 \mathrm{M})$ and $1 \mathrm{~mL}$ iodine in $\mathrm{MeOH}(0.2 \mathrm{M})$ were added to the mixtures and left to react for $1 \mathrm{~h}$ at room temperature. To quench the excess of iodine, $1 \mathrm{~mL}$ of ascorbic acid (1 M) in water was added to the reaction flasks followed by addition of an excess of tris(2-carboxyethyl)phosphine (TCEP) $(1 \mathrm{mmol})$ to reduce the formed disulfide bonds and the mixture was stirred overnight. Finally, the mixtures were dialyzed against water for 2 days at room temperature (MWCO, $10 \mathrm{kDa}$ ) and the products were obtained after lyophilization. The obtained polymers were characterized by ${ }^{1} \mathrm{H}-\mathrm{NMR}$ (PNC: SI-Fig. 1 A-B, mPNC: SI-Fig. 2 A-B) and GPC (PNC: SI-Fig. 5 A, and mPNC: SI-Fig. 5 C) analyses.

\subsection{Synthesis of P(NIPAM-co-HPMA-ETSA)-PEG-P(NIPAM-co-HPMA-ETSA), PNE and m-PEG-P(NIPAM-co-HPMA-ETSA), m-PNE}


PNE and mPNE were synthesized using poly(ethylene glycol) bis(2-bromoisobutyrate) ${ }^{40}$ (50 $\mathrm{mg}, 7.9 \mu \mathrm{mol}$ ) and methoxy polyethylene glycol 2-bromoisobutyrate (50 mg, $16 \mu \mathrm{mol}$ ) ATRP macroinitiators ${ }^{39}$, respectively. In addition to the macroinitiator, $\mathrm{CuBr}(4.5 \mathrm{mg}, 0.031 \mathrm{mmol})$, $\mathrm{CuBr}_{2}$ (4.7 mg, $0.021 \mathrm{mmol}$ ), NIPAM (268.9 mg; $2.38 \mathrm{mmol}$ ) and HPMA-ETSA (55 mg, 0.16 mmol) were dissolved in a mixture of $2.5 \mathrm{~mL}$ water, $0.6 \mathrm{~mL}$ acetonitrile and $1.3 \mathrm{~mL}$ of DMSO. The mixtures were stirred and deoxygenated by flushing with nitrogen for $15 \mathrm{~min}$. at room temperature and $15 \mathrm{~min}$. in an ice bath. Next, polymerizations were started by the addition of $16 \mu \mathrm{L}(0.06 \mathrm{mmol})$ of Me 6 TREN after which The color of the mixtures changed to blue and the reaction mixtures were stirred for $5 \mathrm{~h}$ in an ice bath. The crude products were diluted with water (final volume $15 \mathrm{~mL}$ ) and subsequently dialyzed (MWCO, $10 \mathrm{kDa}$ ) against water at room temperature for two days and the products were obtained after lyophilization and characterized using ${ }^{1} \mathrm{H}-\mathrm{NMR}$ analysis (PNE: SI-Fig. 3, mPNE: SI-Fig. 4) and GPC (PNE: SI-Fig. 5 B, mPNE SI-Fig. 5 D) analyses.

\section{4. ${ }^{1} \mathrm{H}-\mathrm{NMR}$ Spectroscopy}

The obtained polymers were characterized by ${ }^{1} \mathrm{H}-\mathrm{NMR}$ using a Bruker 600 UltraShield ${ }^{\mathrm{TM}}$ spectrometer (Billerica, Massachusetts, USA). The chemical shifts were calibrated against the residual solvent peak of $\mathrm{CHCl}_{3}(\delta=7.26 \mathrm{ppm})$.

\subsection{Gel Permeation Chromatography (GPC)}

The molecular weight and polydispersity index of the synthesized polymers were determined by GPC using a Waters Alliance 2414 System (Waters Corporation, Milford, MA) equipped with a refractive index detector. Samples were dissolved in DMF containing 10 $\mathrm{mM} \mathrm{LiCl}$ at a concentration of $5 \mathrm{mg} \cdot \mathrm{mL}^{-1}$ with an injection volume of $50 \mu \mathrm{L}$. The separation was performed using the same solvent as eluent using 2 PLgel $5 \mu \mathrm{m}$ Mixed-D columns (Polymer Laboratories, UK) at a temperature of $65^{\circ} \mathrm{C}$ with a flow of $1 \mathrm{~mL}$. $\mathrm{min}^{-1}$. A series of linear PEGs with narrow and defined molecular weights were used as calibration standards ${ }^{44}$. Data were recorded and analyzed with Empower software v. 3.

\subsection{Determination of Cloud Point}

The cloud point (CP) of the synthesized polymers was determined on a Jasko FP-8300 spectrofluorometer (JASCO, Tokyo, Japan). The wavelength was set at $650 \mathrm{~nm}$ and the CP is defined as the onset of increasing scattering intensity. Samples were dissolved at a 
concentration of $1 \mathrm{mg} \cdot \mathrm{mL}^{-1}$ in PBS and heated from 10 to $50{ }^{\circ} \mathrm{C}$ with a heating rate of $1^{\circ} \mathrm{C}$ per minute.

\subsection{Micelle Formation}

Polymers, (m)PNC and (m)PNE, were separately dissolved at a concentration of $20 \mathrm{mg} \cdot \mathrm{mL}^{-1}$ at $4{ }^{\circ} \mathrm{C}$ for $3 \mathrm{~h}$. Subsequently, the obtained solutions of (m)PNC and (m)PNE were mixed in a volume ratio of 1:1 (i.e. 1:1 molar ratio of HPMA-Cys to HPMA-ETSA) and quickly heated up to $50{ }^{\circ} \mathrm{C}$ using an oil bath and stirred at this temperature overnight. The resulting core crosslinked micelles were purified using a PBS equilibrated PD-10 desalting column according to the method provided by the supplier.

\subsection{Preparation of the Fluorescently Labeled Micelles}

Micelles were prepared and purified as described in section 2.7 and subsequently, $50 \mu \mathrm{L}$ of a $20 \mu \mathrm{g} \cdot \mathrm{mL}^{-1}$ maleimide-Alexa fluor C5 750 solution in DMSO was added to $5 \mathrm{~mL}$ of the micelle dispersion (20 mg. $\mathrm{mL}^{-1}$ ) in PBS and left to react at $4{ }^{\circ} \mathrm{C}$ for 2 days (the maleimide group of the dye reacts with residual thiol groups present in the micellar core after native chemical ligation or of non-reacted cysteine moieties). Next, the labeled micelles were purified three times by PD-10 desalting columns equilibrated with deionized water according to the method provided by the supplier. Finally, the concentration of micellar dispersions was adjusted to $15 \mathrm{mg} \cdot \mathrm{mL}^{-1}$ in PBS. The dispersions were filtered with a $0.45 \mu \mathrm{m}$ syringe filter (Whatman, GE Healthcare Life Sciences) prior to injection in mice.

\subsection{Zeta-potential}

The micellar dispersions in PBS were diluted (1:40 v/v) with a $20 \mathrm{mM} \mathrm{HEPES,} \mathrm{pH} 7.4$ (final concentration $\left.0.5 \mathrm{mg} \cdot \mathrm{mL}^{-1}\right)$. The zeta-potential of the micelles was measured using a Zetasizer Nano-Z (Malvern Instruments Ltd., Malvern, UK).

\subsection{Dynamic Light Scattering (DLS)}

The diameters of the different micelles were determined by DLS using a Zetasizer Nano $S$ (ZEN 1600) (Malvern Instruments Ltd., Malvern, UK) equipped with a He-Ne $4 \mathrm{~mW}, 632.8$ $\mathrm{nm}$ laser. The measurements were carried out at a $173^{\circ}$ angle and at $37^{\circ} \mathrm{C}$ controlled by instrument; the solvent viscosity was corrected for this temperature by the software. The 
Z-average diameter and polydispersity index were calculated by the Zetasizer software $\mathrm{v}$. 7.13 .

\subsection{Static Light Scattering (SLS)}

The weight-average molecular weight, aggregation number $\left(\mathrm{N}_{\text {agg }}\right)$ and the radius of gyration of the micelles were determined by SLS using an ALV7004 correlator, ALV/LSE-5004 Goniometer, ALV/Dual High QE APD detector unit (Langen, Germany) with fiber splitting device with a set-up of 2 off detection system. A uniphase Model 1145P He-Ne Laser was used at the laser wavelength of $632.8 \mathrm{~nm}$ and power of $22 \mathrm{~mW}$. The temperature was controlled by a Julabo (Seelbach, Germany) CF41 thermostatic bath and viscosity was corrected by the software.

\subsection{Asymmetrical Flow Field-Flow Fractionation (AF4) and Multi-Angle Light Scattering (MALS)}

AF4 experiments were performed using an AF2000 system (Postnova Analytics, Salt Lake City, UT) coupled with an SPD-20A UV/Vis detector operated PN3212 at $280 \mathrm{~nm}$ (Shimadzu, Kyoto, Japan), a refractive-index detector PN3150 (Postnova Analytics, Landsberg am Lech, Germany) and a multi-angle light scattering (MALS) detector PN3070 (Postnova Analytics). The system was equipped with an analytical AF4 channel (Postnova Z-AF4-CHA-611) using a $350 \mu \mathrm{m}$ spacer. A regenerated cellulose membrane (MWCO, $10 \mathrm{kDa}$ ) (Z-AF4-MEM-603$10 K D$, Postnova Analytics) was used as the accumulation wall. The temperature of the channel was kept at $25{ }^{\circ} \mathrm{C}$ during the measurements. Data acquisition was carried out by AF2000 control software v. 2.1.0.1 (Postnova Analytics). The molar mass and radius of the micelles were calculated after subtraction of the blank using the ( $2^{\text {nd }}$ degree) Berry model. The measurements were carried out in PBS buffer containing $0.05 \% \mathrm{NaN}_{3}$. Micellar dispersions in PBS diluted to a final concentration of $4.5 \mathrm{mg} \cdot \mathrm{mL}^{-1}$ with human serum (1:1 v/v) either positive for anti-PEG IgM and IgG (+ Serum) or negative for anti-PEG antibodies (-Serum) and incubated at $37^{\circ} \mathrm{C}$ for $0,1,6$, and $24 \mathrm{~h}$. Serum samples (+ or - ) were also diluted to $1: 1 \mathrm{v} / \mathrm{v}$ with PBS prior to the injections. The injection volume was $10 \mu \mathrm{L}$ and the detector flow was kept at $0.5 \mathrm{~mL}$. $\mathrm{min}^{-1}$. After injection at a flow of $0.20 \mathrm{~mL}^{\mathrm{min}} \mathrm{m}^{-1}$, the sample was focused for $4 \mathrm{~min}$. at a focusing flow rate of $2.3 \mathrm{~mL} \cdot \mathrm{min}^{-1}$ and with a cross-flow (CF) of $2 \mathrm{~mL}$. $\mathrm{min}^{-1}$. After focusing, the profile of the CF was kept constant at $2 \mathrm{~mL} \cdot \mathrm{min}^{-1}$ for $7 \mathrm{~min}$ followed by a time-delayed exponential decay (TDE; 0.5 exponent decay) over a $20 \mathrm{~min}$ period until CF reached $0.1 \mathrm{~mL}$. $\mathrm{min}^{-1}$. The CF was then kept at $0.1 \mathrm{~mL} \cdot \mathrm{min}^{-1}$ for $15 \mathrm{~min}$. Lastly, 
as a rinsing step, the CF was turned to zero and only the laminar detector flow of $0.5 \mathrm{~mL}$. $\mathrm{min}$

${ }^{1}$ was kept for $10 \mathrm{~min}$. Cross-flow programmed with a TDE was found optimal to separate the serum proteins and the micelles.

\subsection{Animal Experiments}

The animal study was approved by the animal experimental committee of Utrecht University, the Netherlands. Both female and male (2:1), 8-10 weeks old immunocompetent BALB/c mice were used (Envigo, the Netherlands). The mice were kept in standard housing with standard rodent chow and water available ad libitum at a $12 \mathrm{~h}$ light/dark cycle.

\subsection{Circulation Kinetics and Biodistribution of Fluorescently Labeled Micelles}

The mice were divided into different groups and the experimental design is depicted in figure $2 \mathrm{~A}$. At the first time point, all mice received a single intravenous (i.v.) injection of 100 $\mu \mathrm{L}$ of either flower- or star-like micelles at a concentration of $15 \mathrm{mg} \cdot \mathrm{mL}^{-1}$ in PBS. After administration, blood was collected at the following time points: $1 \mathrm{~min}$. (100\% injection control), $1 \mathrm{~h}, 4 \mathrm{~h}$, and $24 \mathrm{~h}$ via submandibular puncture $(50-80 \mu \mathrm{L})$ in ethylenediaminetetraacetic acid (EDTA) blood tubes (SARSTEDT, Germany). From three mice of each group, blood was drawn after $24 \mathrm{~h}$ via cardiac puncture under deep isoflurane anesthesia and finally, the animals were sacrificed by cervical dislocation. Major organs (brain, lungs, kidneys, liver, spleen, and heart) from the mice were collected and imaged for whole organ fluorescence using a Pearl ${ }^{\circledR}$ impulse imager (LI-COR, Lincoln, Nebraska, USA). Finally, the mentioned organs were snap-frozen in liquid nitrogen and stored at $-80^{\circ} \mathrm{C}$ until further analysis.

For the second injection, seven days after the first injection, the groups that first received either flower- or star-like micelles were divided into two subgroups receiving a second i.v. injection of $100 \mu \mathrm{L}$ of either flower- or star-like micelles at an equal concentration (15 $\mathrm{mg} \cdot \mathrm{mL}^{-1}$ ) (see schedule figure $2 \mathrm{~A}$ ). Subsequently, the same method of blood sampling was performed and after $24 \mathrm{~h}$ tissues were collected, imaged and stored at $-80^{\circ} \mathrm{C}$. Plasma was prepared by centrifugation at $1000 \times \mathrm{g}$ for $10 \mathrm{~min}$. at $4{ }^{\circ} \mathrm{C}$ and stored at $-20{ }^{\circ} \mathrm{C}$ prior to analysis.

The groups are abbreviated as follows: the group that received star-like micelles in the first injection: S, flower-like micelles in the first injection: $\mathbf{F}$, flower-like micelles in both injections: FF, star-like micelles in both injections: SS, flower-like micelles in the first and 
star-like micelles in the second injection: FS, and finally star-like micelles in the first and flower-like micelles in the second injection: SF.

To assess the accumulation of fluorescently-labeled micelles in the isolated organs, parts of frozen organs were weighed and homogenized in PBS buffer (100 mg tissue/100 $\mu \mathrm{L}$ PBS). For this purpose, a Precellys 24, (Bertin Instruments, Montigny-le-Bretonneux, France) bead mill homogenizer with a rotation speed of $6000 \mathrm{rpm}$ for $60 \mathrm{~s}$ was used. The homogenates were centrifuged for $10 \mathrm{~min}$. at $12,000 \times \mathrm{g}$ and at $4{ }^{\circ} \mathrm{C}$, and subsequently, the fluorescence intensity in the supernatants was measured using an Odyssey ${ }^{\circledR}$ scanner (LI-COR, Lincoln, Nebraska, USA). The same instrument was used for the detection of the fluorescence intensity in plasma. A calibration curve of spiked amounts of the corresponding dyeconjugated micelles in plasma was used to assess the concentration of micelles.

For histological examination of the collected organs, the remaining parts of the frozen tissues were cryosectioned with $5 \mu \mathrm{m}$ thickness Leica CM1950 cryostat (Leica Biosystems, Wetzlar, Germany). The sections were fixed in $100 \%$ methanol for $15 \mathrm{~min}$. at room temperature. Subsequently, nuclei and the actin cytoskeleton were stained at room temperature with a $1 \mu \mathrm{g} \cdot \mathrm{mL}^{-1}$ solution of HOECHST 33342 in PBS for $15 \mathrm{~min}$. and phalloidinAlexa 488 (1:50) in PBS for 45 min., respectively. Next, the slides were imaged using a $60 \times$ 1.49NA Nikon oil objective (Nikon Instruments Inc., New York, USA). For HOECHST staining, a 330-380 $\mathrm{nm}$ excitation filter, a $400 \mathrm{~nm}$ longpass dichroic mirror and a $420 \mathrm{~nm}$ longpass emission filter (Nikon UV-2A filterset) was used. A 450-490 nm excitation filter, a $505 \mathrm{~nm}$ longpass dichroic mirror and a $520 \mathrm{~nm}$ longpass emission filter (Nikon B-2A filterset) was used for Alexa 488 staining. And eventually, a 690-730 nm excitation filter, a $741 \mathrm{~nm}$ longpass dichroic mirror and a 750-800 nm emission filter (Semrock Cy7-A-NTE-ZERO filterset) were applied for Alexa 750-labeled micelles. The images were recorded with an Andor NEO sCMOS camera and were analyzed using ImageJ software v. 10.2.

\subsection{Determination of Anti-PEG Antibodies Using ELISA}

To determine the concentrations of anti-PEG antibodies (IgG and IgM) in plasma, 96 well plates (Greiner, 655101) were coated overnight with a solution of PEGylated granulocyte colony-stimulating factor (PEG-G-CSF; $1 \mu \mathrm{g} \cdot \mathrm{mL}^{-1}$ ) at $4^{\circ} \mathrm{C}$. Plates were then blocked for $2 \mathrm{~h}$ at room temperature with a $2 \%$ solution of bovine serum albumin (BSA) in PBS. Mouse plasma was added to the wells at different dilutions. The plate was incubated at room temperature for $1.5 \mathrm{~h}$ and washed five times with a $0.05 \%$ solution of 3-[(3cholamidopropyl)dimethylammonio]-1-propanesulfonate (CHAPS) in PBS. Next, $100 \mu \mathrm{L}$ of detection antibodies of either horseradish peroxidase (HRP)-conjugated anti-mouse IgG 
(2000 times in 2\% BSA/PBS) or biotinylated anti-mouse IgM (diluted 8000 times in $2 \%$ BSA/PBS) were added. Subsequently, the plate was incubated for $1 \mathrm{~h}$ at room temperature and then washed five times. Streptavidin-horseradish peroxidase (SA-HRP; $100 \mu \mathrm{L}$, diluted 1000 times in $2 \%$ BSA/PBS) was added to the wells containing biotinylated anti-mouse IgM. Only PBS was added to the wells containing HRP-conjugated anti-mouse IgG and then the plate was incubated for $30 \mathrm{~min}$. at room temperature. Binding of anti-PEG was visualized by the addition of $100 \mu \mathrm{L}$ of $3,3^{\prime}, 5,5^{\prime}$-tetramethylbenzidine (TMB) to the plates and incubated for 12 or $5 \mathrm{~min}$. in the case of IgG or IgM detection, respectively. Next, $100 \mu \mathrm{L}$ of a $1 \mathrm{M} \mathrm{HCl}$ was added to stop the reaction. The optical density (OD) was measured on an iMark ELISA reader (Bio-Rad Laboratories Inc., Hercules, CA) at $450 \mathrm{~nm}$. To check for the specificity of the binding, 1\% mPEG ( $\mathrm{Mn}_{\mathrm{n}}$ of $5 \mathrm{kDa}$ ) was added as a competitor.

The binding of both types of micelles to commercial anti-PEG antibodies was also studied. In this assay rabbit monoclonal antibodies, an IgM isotype specific for the PEG backbone (ab133471) and an IgG isotype specific for the methoxy group of PEG (ab190652) were used. The two different micelles were diluted in $10 \% \mathrm{BALB} / \mathrm{c}$ mouse plasma (negative for anti-PEG antibodies) in 2\%BSA/PBS, with the final concentrations of $0.05,0.1,0.2$ and $0.4 \mathrm{mg} \cdot \mathrm{mL}^{-1}$. A negative control was taken along without any micelles. In these mixtures, the commercially available anti-PEG antibodies were added with final concentrations ranging from 3.7-100 ng. $\mathrm{mL}^{-1}$. These samples were incubated for $1 \mathrm{~h}$ at $37^{\circ} \mathrm{C}$ before adding to PEG-G-CSF coated plates. Subsequently, the plates were incubated at room temperature for $1.5 \mathrm{~h}$ and washed five times with $0.05 \%$ CHAPS/PBS. The anti-PEG antibodies were detected with HRPconjugated swine anti-rabbit Ig. The rest of the protocol was followed as described above.

\subsection{Statistical Analysis}

The statistical differences between the experimental groups were determined by a student test with two-tailed distribution using the software GraphPad Prism 5 (GraphPad Software Inc., La Jolla, California). p values $<0.05$ were considered as statistically significant.

\section{Results and Discussions}

\subsection{Polymer Synthesis and Characterization}

Two complementary $A B A$ and $A B$ block copolymers were synthesized using atom transfer radical polymerization (ATRP) ${ }^{45}$. An overview of polymer structures is shown in figure 1 and the polymer characteristics are summarized in table 1 . The B-block in both types of polymers was PEG with a number average molecular weight $\left(M_{n}\right)$ of 3 and $6 \mathrm{kDa}$ in $A B$ and 
$A B A$, respectively. The PEG initiator used for the preparation of the $A B$ polymers carried an ATRP initiator on one terminal end whereas the other end was capped with a methoxy group. The PEG macroinitiator used for ABA polymerization carried ATRP initiators at both ends. The composition of A-blocks was either a copolymer of NIPAM and HPMA-Cys or NIPAM and HPMA-ETSA, both synthesized at a feed molar ratio of 93:7. This strategy was chosen to obtain $A B$ block copolymers with half-size of $A B A$ block copolymers and similar comonomer compositions. After polymerization and purification, the ABA copolymers of P(NIPAM-Co-HPMA-CYS)-PEG-P(NIPAM-Co-Boc-HPMA-Cys-(Acm)) (PNC) and P(NIPAM-coHPMA-ETSA)-PEG-P(NIPAM-CO-HPMA-ETSA) (PNE) were obtained with a yield of 94 and 85\%, respectively. The AB copolymers of methoxy-PEG-P(NIPAM-co-HPMA-Boc-Cys-(Acm)) (mPNC) and methoxy-PEG-P(NIPAM-co- HPMA-ETSA)(mPNE) were obtained with similar yields of 97 and $89 \%$, respectively. As shown in table 1, the comonomer compositions of the A blocks in the obtained polymers were, within the experimental error, equal to the feed ratios. GPC analysis showed that the Mn's of mPNC (21.4 kDa) and mPNE (21.7 kDa) were half of PNC (42.1 kDa) and PNE (40.9 kDa), as expected. The obtained polymers showed PDIs of 1.4-1.8, which is similar as observed before for block copolymers containing monomers with different reactivities and synthesized by ATRP techniques ${ }^{46}$. Table 1 shows that higher values of $\mathrm{Mn}_{n}$ as determined by GPC than Mn's obtained by NMR analysis were found which can be attributed to aggregation of PNIPAM due to inter- and intramolecular hydrogen bonding ${ }^{47,48}$. The Boc and Acm protecting groups of cysteines in the protected PNC and $\mathrm{mPNC}$ were removed to generate free thiol and amine groups in cysteine moieties required for native chemical ligation (NCL) ${ }^{49}$ in the micellar core (SI-Fig. 1 B and 2 B). The different polymers exhibited a cloud point $(\mathrm{CP})$ around $30{ }^{\circ} \mathrm{C}$ in aqueous solution as expected for polymers containing a PNIPAM rich block ${ }^{50}$ (Table 1 ). 


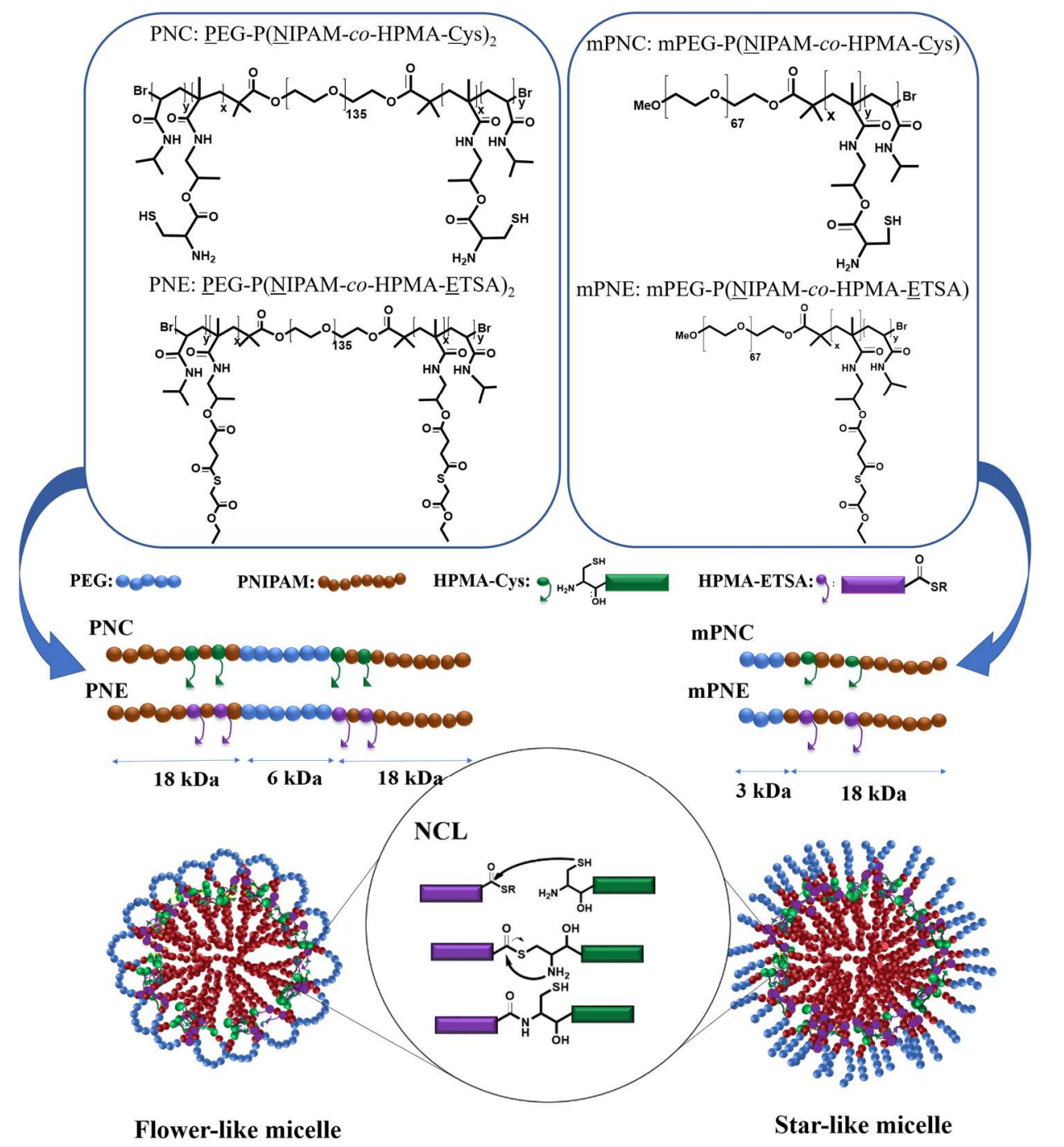

Figure 1: Schematic overview and structures of PNC, PNE, mPNC, and mPNE. PNC and PNE were used for the formation of flower-like micelles, whereas mPNC and MPNE were used to prepare star-like micelles. Native chemical ligation above the LCST of the thermosensitive PNIPAM block stabilized the micellar structure ${ }^{40}$. 
Table 1: Characteristics of the ABA triblock copolymers of protected PNC and PNE and AB diblock copolymers of protected mPNC and mPNE synthesized by ATRP. ABA and AB polymers contain PEG of 6 and $3 \mathrm{kDa}$ as $\mathrm{B}$ block, respectively. The thermosensitive blocks of different polymers are composed of either NIPAM and HPMA-ETSA in (PNE, mPNE) or NIPAM and HPMA-Boc-Cys-(Acm) (protected PNC and mPNC). The feed molar ratios of NIPAM to HPMA-Boc-Cys-(Acm) or HPMA-ETSA were 93:7.

\begin{tabular}{|c|c|c|c|c|c|c|c|}
\hline \multirow{2}{*}{ Polymer } & \multicolumn{2}{|c|}{ Composition of the thermosensitive B blocks ${ }^{a}$} & \multirow{2}{*}{$\begin{array}{c}\mathrm{Mn}^{\mathrm{a}} \\
\text { (kDa) }\end{array}$} & \multirow{2}{*}{$\begin{array}{c}\mathrm{M}_{\mathrm{n}}^{\mathrm{b}} \\
(\mathrm{kDa})\end{array}$} & \multirow{2}{*}{$P D I^{b}$} & \multirow{2}{*}{$\begin{array}{c}\mathrm{CP} \\
\left({ }^{\circ} \mathrm{C}\right)\end{array}$} & \multirow{2}{*}{$\begin{array}{c}\text { Yield } \\
\text { (\%) }\end{array}$} \\
\hline & [NIPAM]:[HPMA-Boc-Cys-Acm] & [NIPAM]: [HPMA-ETSA] & & & & & \\
\hline PNC & $92: 8$ & - & 42.1 & 69.1 & 1.5 & $34.1^{\mathrm{C}}$ & 94 \\
\hline PNE & - & $92: 8$ & 40.9 & 63.9 & 1.8 & 29.2 & 85 \\
\hline $\mathrm{mPNC}$ & $92: 8$ & - & 21.4 & 34.5 & 1.4 & $32.9^{c}$ & 97 \\
\hline mPNE & - & $93: 7$ & 21.7 & 40.5 & 1.7 & 29.0 & 89 \\
\hline
\end{tabular}

${ }^{\mathrm{a}}$ Determined by ${ }^{1} \mathrm{H}-\mathrm{NMR} .{ }^{\mathrm{b}}$ Determined by GPC. ${ }^{\mathrm{c}}$ Cloud point of the deprotected polymer

\subsection{Micelle Formation and Characterization}

AB block copolymers of MPNC and MPNE were used for the formation of star-like, whereas ABA block copolymers of PNC and PNE were used for the formation of flower-like micelles by a method reported before ${ }^{40}$. Thermosensitive self-assembly of $A$ blocks in $A B$ and $A B A$ polymers resulted in the formation of micelles with a PEG shell and a thermosensitive PNIPAM core. During the micellization, cysteine (in HPMA-Cys) and thioester (in HPMAETSA) moieties were brought in close proximity in the thermosensitive core, which facilitated covalent crosslinking by native chemical ligation. Crosslinking of micelles is known to improve the stability of micelles in body fluids ${ }^{5,51}$. The obtained flower-like micelles showed a Z-average diameter of $65 \pm 2 \mathrm{~nm}$ (PDI 0.1), and $\zeta$-potential of $-4.2 \pm 0.4 \mathrm{mV}$. The star-like micelles were slightly larger with a Z-average of $83 \pm 2 \mathrm{~nm}$ (PDI 0.05), and a $\zeta$ potential of $-5.9 \pm 0.2$. SLS characterizations (table 2 ) revealed a higher aggregation number $\left(\mathrm{N}_{\text {agg }}\right.$ ) for star-like micelles than for flower-like micelles ( 1700 vs. 400 ) comparable to what has been reported for similar micelles ${ }^{39}$. The lower $\mathrm{N}_{\text {agg }}$ for flower-like micelles is most likely due to the more surface area occupied by PEG chains in a loop conformation as compared to linear PEG in star-like micelles ${ }^{39}$. This results in a higher surface area per PEG chains (SA/PEG) in flower-like micelles than star-like micelles ( 37 vs. $12 \mathrm{~nm}^{2}$ ). The low value of SA/PEG for star-like micelles $\left(\sim 12 \mathrm{~nm}^{2}\right)$ indicates that the surface has a high PEG grafting density and that the PEG chains are in a highly stretched "brush" conformation ${ }^{36,44}$. The SA/PEG value for flower-like micelles is larger ( 37 vs. $12 \mathrm{~nm}^{2}$ ) implying a lower PEG grafting intensity. In such structure, PEG chains show low flexibility in a loop conformation meaning 
they are highly stretched in a "mushroom" conformation ${ }^{39}$. The ratios of radius of gyration $\left(R_{g}\right)$ to hydrodynamic radius $\left(R_{h}\right)\left(R_{g} / R_{h}\right)$ in both types of micelles were, within the experimental error, similar $(0.86 \pm 0.04$ and $0.92 \pm 0.01)$, and slightly higher than the value of 0.775 for hard spherical particles ${ }^{52,53}$. Both types of micelles showed slightly negative $\zeta-$ potential $(-4.2 \pm 0.4$ and $-5.9 \pm 0.2$ for flower-and star-like micelles, respectively), which is in agreement with what has been reported for the $\zeta$-potential of other PEGylated nanoparticles ${ }^{54}$. To investigate structural changes and/or possible aggregation of star-, and flower-like micelles upon incubation with human serum either positive for anti-PEG IgM and IgG (+ Serum) or negative (- Serum), asymmetrical flow field-flow fractionation (AF4) was used. No significant differences in the retention times and accordingly radius of micelles were observed indicating no micelles aggregation (SI-Fig. 7, SI-table 1).

Table 2: Characteristics of core crosslinked flower- and star-like micelles at $37^{\circ} \mathrm{C}$ as determined by dynamic and static light scattering (DLS and SLS).

\begin{tabular}{|c|c|c|c|c|c|c|c|}
\hline Micelle & $\begin{array}{c}\mathrm{R}_{\mathrm{g}} \\
(\mathrm{nm})\end{array}$ & $\begin{array}{l}\mathrm{R}_{\mathrm{h}} \mathrm{b} \\
(\mathrm{nm})\end{array}$ & $\mathrm{R}_{\mathrm{g}} / \mathrm{R}_{\mathrm{h}}$ & $\begin{array}{c}M_{w}{ }^{C} \\
\left(10^{6} \mathrm{Da}\right)\end{array}$ & $\mathrm{N}_{a g g}{ }^{\mathrm{d}}$ & $\begin{array}{c}\text { SA/PEG } \\
\left(\mathrm{nm}^{2}\right)\end{array}$ & $\begin{array}{c}\zeta \text {-potential } \\
(\mathrm{mV})\end{array}$ \\
\hline Flower-like & $29.3 \pm 1.2$ & $33.8 \pm 0.4$ & $0.86 \pm 0.04$ & $14.7 \pm 3.4$ & $389 \pm 9$ & $36.9 \pm 1.7$ & $-4.2 \pm 0.4$ \\
\hline Star-like & $37.0 \pm 1.0$ & $40.3 \pm 0.3$ & $0.92 \pm 0.01$ & $36.3 \pm 1.2$ & $1690 \pm 60$ & $12.1 \pm 0.6$ & $-5.9 \pm 0.2$ \\
\hline
\end{tabular}

${ }^{a}$ Radius of gyration determined by SLS, ${ }^{b}$ Hydrodynamic radius determined by DLS, ${ }^{c}$ molecular weight of micelles. (The calculation methods for $R_{g}, R_{h}$, and molecular weight are shown in supporting information section SLS). ${ }^{d}$ Aggregation number of the micelles.

\subsection{Circulation Kinetics of Star- and Flower-like Micelles}

Fluorescently labeled micelles were prepared by conjugation of maleimide functionalized Alexa Fluor ${ }^{\mathrm{TM}} 750$ to residual thiol moieties after native chemical ligation in the micellar core via maleimide-thiol conjugation chemistry ${ }^{55}$. The obtained micelles were extensively purified to remove non-conjugated dye. The resulting fluorescently labeled micelles

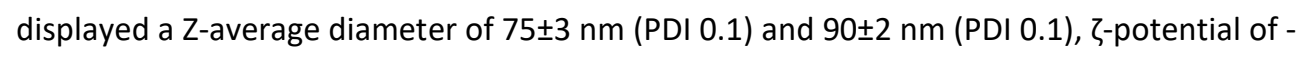
$4.8 \pm 0.3$ and $-5.0 \pm 0.6$ for flower-, and star-like micelles at $37{ }^{\circ} \mathrm{C}$, respectively. The slight increase in the size of micelles after dye conjugation ( $65 \pm 2$ vs. $75 \pm 3$ for flower-like micelles and $83 \pm 2$ vs. $90 \pm 2$ for star-like micelles) can be explained by repulsion of negative charges of the coupled dye molecules in the core of the micelles. The labeled micelles were used to investigate the effect of PEG conformation on circulation kinetics and tissue distribution in immunocompetent BALB/c mice. Additionally, to study the possible occurrence of the so- 
called $A B C$ phenomenon ${ }^{18,22}$, a second injection was given 7 days after the first injection (Fig. 2 A). This timing was chosen based on publications stating that the concentration of anti-PEG IgM in circulation peaks around 5 to 10 days after the first administration of PEGylated therapeutics in mice ${ }^{56,57}$. For the second injection, each group of flower- and star-like micelles was divided into two subgroups to receive either flower- or star-like micelles. The results are depicted in figure $2 \mathrm{~B}$ (first injection) and C (second injection). It was observed that in group $F, 32 \pm 10 \%$ of the injected dose (I.D.) was in the circulation $24 \mathrm{~h}$ after injection, while in group S, $13 \pm 4 \%$ of I.D. was detected at this time point (Fig. 2 B, SIFig. 6 A). The circulation data were used to calculate circulation half-life $\left(t_{1 / 2}\right)$ by applying non-compartmental pharmacokinetic model using $\mathrm{PKsolver}^{58}$. The $\mathrm{t}_{1 / 2}$ of flower-like micelles $(18.6 \pm 3.1 \mathrm{~h})$ was significantly longer $(p<0.05)$ than the $t_{1 / 2}$ of star-like micelles $(10.7 \pm 0.7 \mathrm{~h})$ (Fig. 2 D, SI-Fig. 6 C). Particle size is an important factor for circulation kinetics and biodistribution of (pegylated) nanoparticles ${ }^{59}$. Sub $\sim 100 \mathrm{~nm}$ pegylated nanoparticles have relatively long circulation times and high tumor accumulation ${ }^{60}$. The presence of fenestrated endothelium ( $60 \mathrm{~nm}$ ) in glands, digestive mucosa, kidneys, and discontinuous endothelium in liver and spleen ( $50-100 \mathrm{~nm}$ ) allows nanoparticles to selectively pass through theses organs ${ }^{61}$. Besides particle size, it has been demonstrated in many papers that the PEG density also affects circulation kinetics. Nanoparticles of equal size but with higher PEG density circulate generally longer than nanoparticles with low PEG grafting density $^{15,35,62,63}$. In this study, the investigated flower-like micelles were slightly smaller than star-like micelles (Z-average of dye conjugated micelles $75 \pm 3$ vs. $90 \pm 2 \mathrm{~nm}$ ), however, both types are sub $100 \mathrm{~nm}$. In contrast, the PEG density on the surface of star-like micelles is substantially higher than that of flower-like micelles (SA/PEG of $37 \mathrm{vs.} 12 \mathrm{~nm}^{2}$, table 2). Based on previous studies, no significant difference in the circulation kinetics of nanoparticles in this size range (having similar zeta potential) can be expected ${ }^{15,35,64}$. The longer circulation time of flower-like micelles can therefore be explained by the reduced flexibility of PEG in the loop structure ${ }^{39}$. Likely, such conformation presents a steric barrier, which is kinetically less favorable for protein adsorption than linear (brush-like) PEG in the corona of micelles thereby yielding longer circulation times ${ }^{38}$. 
A
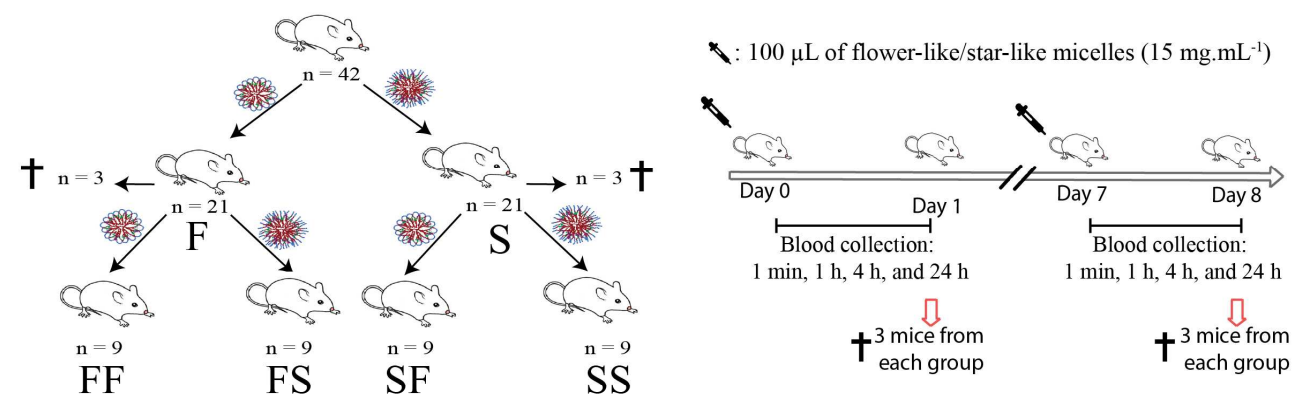

B

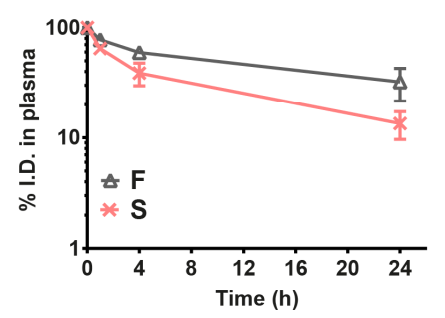

$\mathrm{C}$

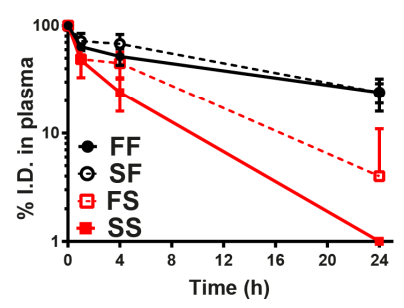

$\mathrm{D}$

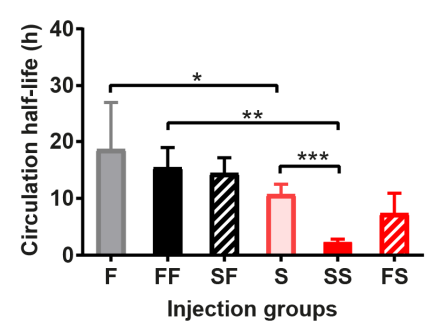

Figure 2: Circulation kinetics of fluorescently labeled (Alexa Fluor ${ }^{\mathrm{TM}} 750$ ) flower-like (F) and star-like (S) micelles investigated in immunocompetent BALB/C mice. A) Schematic representation of the animal study design B) Circulation kinetics of flower-like (in gray) and star-like (in light red) micelles after the first injection. The presented data are mean values of blood samples of 6 mice. C) Circulation kinetics of micelles in mice after the second injection; each data point represents an average of three samples. The concentration of micelles in SS group at $24 \mathrm{~h}$ was below detection limit. D) Circulation half-life $\left(t_{1 / 2}\right)$ calculated based on circulation kinetics of micelles using pharmacokinetics noncompartmental model by means of PKsolver. The error bars are the standard deviations of the mean. $* \mathrm{p}<0.05 ;{ }^{* *} \mathrm{p}<0.01 ; * * * \mathrm{p}<0.001$.

In the FF group, $24 \mathrm{~h}$ after the second injection, $19 \%$ of the I.D. was present in the circulation, while the fluorescence signal for the SS group was below the detection limit meaning that less than $0.1 \%$ of the micelles were in the circulation. After the second injection, micelles in the FF and $\mathbf{S S}$ groups displayed $t_{1 / 2}$ of $15.4 \pm 2.1$ and $2.3 \pm 0.3$, respectively. Notably, a significant difference between $t_{1 / 2}$ of micelles in $\mathbf{S}$ and $\mathbf{S S}$ groups was observed ( $10.7 \pm 0.7$ vs. $2.3 \pm 0.3 \mathrm{~h}, \mathrm{p}=0.0001)$, while $t_{1 / 2}$ of micelles in $\mathbf{F}$ and $\mathbf{F F}$ remained similar (18.6 $\pm 3.1 \mathrm{~h}$ vs. $15.4 \pm 2.2 \mathrm{~h} ; \mathrm{p}=0.56)$.

In the SF and FS groups, after $24 \mathrm{~h}, \sim 22$ and $4 \%$ of the I.D. was detected in the circulation, respectively. The $t_{1 / 2}$ of micelles in $\mathbf{S F}(14.4 \pm 1.1 \mathrm{~h})$ group was not significantly different from the $t_{1 / 2}$ of micelles of the $F$ group $(15.4 \pm 2.2, p=0.44)$. Also, the $t_{1 / 2}$ of star-like micelles in the 
FS group $(7.3 \pm 3.0 \mathrm{~h})$ was not significantly different from $t_{1 / 2}$ of micelles in the $\mathbf{S}(p=0.092)$ or SS $(p=0.073)$ groups. It should be mentioned that in the FS group the concentration of micelles in two mice out of three was below detection whereas one mouse showed $11 \%$ of I.D. $24 \mathrm{~h}$ after the second injection. Taken together, mice that received flower-like micelles and subsequently flower- or star-like micelles (FF and FS groups) showed the same circulation kinetics for flower-like micelles as their first injection (F group), whereas, starlike micelles were cleared rapidly from the circulation in 2 out of 3 mice (FS group). Mice that received star-like micelles and thereafter either flower- or star-like micelles (SF and SS groups) showed rapid clearance of star-like micelles, whereas the circulation kinetics of the flower-like micelles was the same as observed after their first injection (F group) (Fig. 2 CD). The rapid elimination of star-like compared to flower-like micelles can be explained by the $A B C$ phenomenon, which is associated with the induction of anti-PEG antibodies upon administration of the first dose ${ }^{16,19,30}$. Obviously, these formed anti-PEG antibodies do not seem to recognize PEG in the looped conformation as present in the shell of the flower-like micelles. This is discussed in more detail in the next section.

\subsection{Determination of Anti-PEG Antibodies in Mice Plasma Using ELISA}

An ELISA was used to investigate the presence of anti-PEG antibodies in plasma of mice that received the micelles. Samples collected $24 \mathrm{~h}$ after the second injection were used in this assay to quantify anti-PEG IgM and IgG titers in plasma. To check the specificity of the formed antibodies, 1\% free mPEG ( $\mathrm{Mn}_{\mathrm{kDa}} \mathrm{k}$ ) was added as a competitor to the plasma samples. No anti-PEG IgG antibodies were detected in any of the groups $24 \mathrm{~h}$ after the second injection of either flower- or star-like micelles (data not shown), which is not surprising since normally IgG antibodies are detected 3-4 weeks after administration of a vaccine/antigen ${ }^{65}, 66$. Furthermore, many papers have shown that PEG induces the formation of IgM type rather than IgG antibodies ${ }^{16,17,56}$. No anti-PEG IgM titers were detected in plasma of mice that did not receive any types of micelles (Fig. 3 A). Anti-PEG $\operatorname{IgM}$ antibodies were detected in $\mathbf{F F}$ and $\mathbf{S F}$ groups demonstrating that the mice produced anti-PEG IgM upon injection of both types of micelles (Fig. $3 \mathrm{~A}$ ). The anti-PEG IgM titers in the SS and FS groups were very low, which can be ascribed to binding of the produced antiPEG IgM to the micelles and their subsequent clearance (Fig. $3 \mathrm{~A}$, SI-Fig. 8). To investigate interaction of micelles and anti-PEG IgM and IgG antibodies, micelles were added in different concentrations (0.05-0.4 mg. $\mathrm{mL}^{-1}$; concentration of micelles directly after injection is $\sim 1 \mathrm{mg} . \mathrm{mL}^{-1}$; see legend Fig. 3 ) to control mouse plasma (previously tested negatively for IgM and IgG anti-PEG antibodies). Subsequently, they were tested in an ELISA set up with 
commercial anti-PEG IgM and IgG, specific for the PEG backbone and methoxy group, respectively. Figures $3 \mathrm{C}$-D show that in the presence of flower-like micelles, a slight decrease in anti-PEG IgM and IgG titers was observed, independent of micelle concentration. No binding of anti-PEG IgG to flower-like micelles was expected as this antibody was specific to methoxy group (Fig. 3 D). Surprisingly, no binding of anti-PEG IgM to flower-like micelles was observed although this antibody was specific for the PEG backbone (Fig. $3 \mathrm{C}$ ). Likely, the loop conformation of PEG in flower-like micelles prevents effective interaction between micelles and the anti-PEG IgM. In contrast, Fig. $2 \mathrm{E}$ and $\mathrm{F}$ show that addition of star-like micelles resulted in a drastic reduction in titers of both types of anti-PEG antibodies demonstrating that the PEG chains are recognized by both the IgM (specific for the PEG backbone) and IgG (specific for the methoxy end group of PEG) antibodies.

The observed concentrations of micelles and anti-PEG IgM antibodies present in plasma 24 $\mathrm{h}$ after the second injection (Fig. $3 \mathrm{~A}$, SI-Fig. 8) can provide mechanistic insights into the clearance of the micelles (Fig. 3 B). Detection of anti-PEG IgM in the SF group shows that the star-like micelles after the first injection induced the formation of anti-PEG IgM. It has been shown before that the methoxy end group of PEG is crucial for the induction of antiPEG IgM, anti-PEG IgM binding, and complement activation ${ }^{25,26}$. Therefore, injection of starlike micelles resulted in the formation of anti-PEG IgM that are directed against the methoxy end group of PEG. The similar circulation kinetics of the micelles in the $\mathbf{S F}$ and $\mathbf{F}$ groups $(14.4 \pm 1.1$ vs. $18.6 \pm 3.1 \mathrm{~h} ; \mathrm{p}=0.44)$ strongly suggests that the produced anti-PEG IgM antibodies do not bind to flower-like micelles because the PEG-loops lack methoxy groups. In the plasma of mice of the SS group, neither micelles (24 hour after the second injection) nor anti-PEG IgM (8 days after the first injection) were detected. This might indicate that the produced anti-PEG IgM antibodies bind to the star-like micelles causing opsonization of the micelles which in turn triggers the recognition of star-like micelles by the immune system, resulting in faster clearance of the micelles ( $t_{1 / 2}$ of $10.7 \pm 0.7 \mathrm{vs} .2 .3 \pm 0.3 \mathrm{~h}$ in $\mathbf{S}$ vs. SS groups; $p=0.0001$ ) but also of anti-PEG IgM. The strong binding of anti-PEG antibodies to star-like micelles (Fig. 3 E-F) supports the proposed explanation. Detection of anti-PEG IgM in the FF group (Fig. 3 A) demonstrates that flower-like micelles upon intravenous administration induced the production of anti-PEG IgM. However, the produced anti-PEG IgM did not trigger the faster clearance of the second dose of flower-like micelles $\left(\mathrm{t}_{1 / 2}\right.$ in $\mathbf{F}$ and $\mathbf{F F}$ groups are $18.6 \pm 3.1$ and $15.4 \pm 2.2 \mathrm{~h}$, respectively; $p=0.56)$. The mechanism behind this observation is not fully clear and could be hypothesized in number of ways: a) the antiPEG IgM antibodies induced by flower-like micelles have lower affinity than the anti-PEG IgM antibodies induced by star-like micelles; b) the anti-PEG IgM antibodies induced after 
injection of flower-like micelles bind in such a configuration to the micelles that it does not activate complement; c) binding sites in anti-PEG IgM do not match properly with the loop conformation in flower-like micelles due to the low mobility of PEG chains. In the FS group, anti-PEG IgM antibodies were hardly detected (Fig. 3 A, SI-Fig. 8). This can be explained by binding of the produced anti-PEG IgM to star-like micelles and consequently clearance of the micelles in 2 out of 3 mice. It cannot be excluded that in the mouse in which star-like micelles were detected after $24 \mathrm{~h}$ ( $11 \%$ of the I.D.) anti-PEG IgM antibodies were present, however, these were not detected due to the presence of micelles in the plasma sample (SIFig. 8). The anti-PEG IgM formed after injection of flower-like micelles are most likely directed against the ethylene oxide units present in the loop of PEG. As suggested, they do not strongly bind to flower-like micelles yielding the same circulation kinetics as observed after the first injection (Fig. 2 B-D). Surprisingly, these antibodies do bind to the PEG chains of the star-like micelles resulting in the rapid clearance of micelles (in 2 out of 3 mice the concentration of star-like micelles after $24 \mathrm{~h}$ was below detection (SI-Fig. 8)). A possible explanation is that due to the higher PEG density in star-like micelles the avidity of the antiPEG IgM for these micelles is higher than for flower-like micelles. However, further research is required to substantiate this hypothesis. 


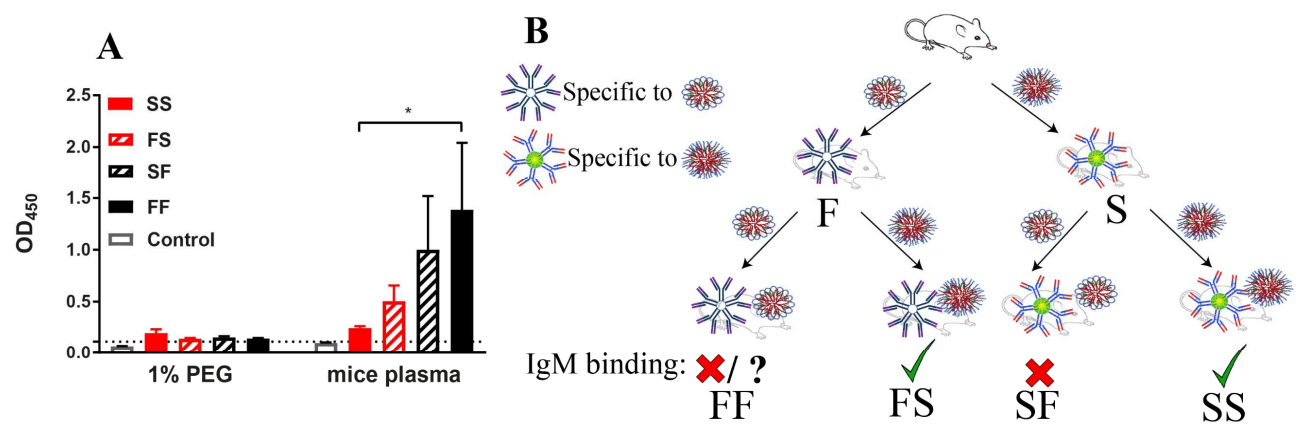

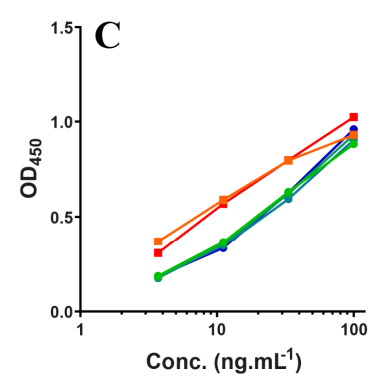

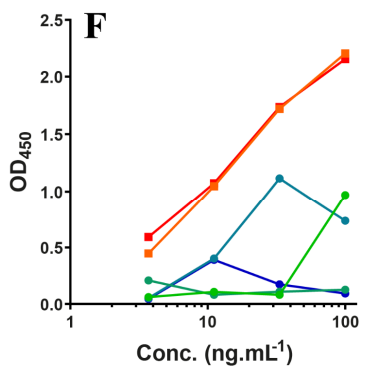

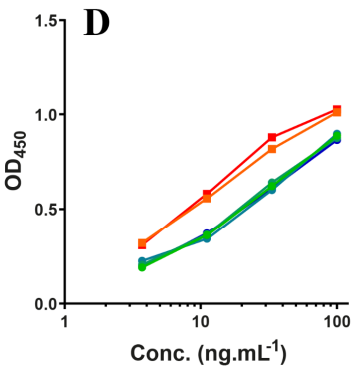

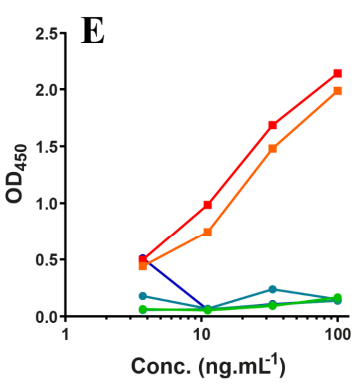

Figure 3: A) Detection of anti-PEG IgM by means of ELISA in plasmas collected from mice $24 \mathrm{~h}$ after the second injection. $1 \% \mathrm{mPEG}(5 \mathrm{kDa})$ was added to determine the specificity of the formed anti-PEG antibodies. BALB/c mice that did not receive any type of micelles were used as control. Data represented mean $\pm S D$ of three samples. ${ }^{*} p<$ 0.05. B) Schematic overview of possible interaction of anti-PEG IgM with flower- and star-like micelles. C) Competition of flower-like micelles with detection of commercial anti-PEG IgM antibody specific for the PEG backbone, D) Competition of flower-like micelles with detection of commercial anti-PEG IgG antibody specific for the methoxy end groups of PEG. E) Competition of star-like micelles with detection of anti-PEG IgM antibody specific to PEG backbone, F) Competition of star-like micelles with detection of anti-PEG IgG antibodies specific to methoxy end groups of PEG. X-axes in C to F refers to the concentration of the commercial anti-PEG antibodies (IgM or IgG). It must be noted that the concentration of micelles in the mice directly after injection was $\sim 1 \mathrm{mg}^{\mathrm{ml}}$ ${ }^{1}$ (100 $\mu \mathrm{L}$ of a $15 \mathrm{mg} \cdot \mathrm{mL}^{-1}$ dispersion was injected, the blood volume of a mouse is $\sim 1.5 \mathrm{~mL}$ ). This means that the lowest concentration of micelles in figures $\mathrm{C}-\mathrm{F}$ of $0.05 \mathrm{mg} \cdot \mathrm{ml}^{-1}$ corresponds with $\sim 5 \%$ of the injected does still in the circulation. 


\subsection{Biodistribution of Flower- and Star-like Micelles After Intravenous Administration}

The biodistribution of flower- and star-like micelles $24 \mathrm{~h}$ after injections was studied by measuring the fluorescence intensity of the micelles in different organs (Fig. 4). After the first injection of flower-like micelles, $1.0 \pm 0.0,9.2 \pm 1.5$, and $3.0 \pm 0.1 \%$ of the injected dose accumulated in the spleen, liver, and kidneys, respectively. In the star-like micelle group, $2.1 \pm 0.1,13.9 \pm 4.3$, and $7.3 \pm 2.4 \%$ of the I.D. accumulated in spleen, liver, and kidneys, respectively. In both groups, micelle accumulation in brain, heart, and lungs was negligible. The relatively low accumulation of both types of micelles seen after the first injection in spleen and liver is in accordance with what has been reported for core crosslinked MPEGb-P((20\% HEMAm-Lac 1$)$-co-(80\%HEMA-Lac 2$)$ micelles of similar size ${ }^{67}$ and can be explained by the dense PEG corona of micelles (SA/PEG was $~ 37$ and $12 \mathrm{~nm}^{2}$ for flower- and star-like micelles, respectively) which prevents their opsonization in comparison with less densely PEGylated nanoparticles (SA/PEG 70-300 $\left.\mathrm{nm}^{2}\right)^{62}$. Further, It has been shown that sub 100 $\mathrm{nm}$ pegylated nanoparticles display reduced plasma protein adsorption on their surface thereby undergoing reduced hepatic filtration ${ }^{68}$. Accumulation of micelles was also observed in the kidneys, which cannot be ascribed to free dye since first, micelles were extensively purified before injection and second, excretion half-life of free hydrophilic dye in mice is around $2.5 \mathrm{~min}^{69}$. Accumulation of micelles in kidneys is in agreement with what has been reported for other nanoparticles with similar sizes. For instance, Davis et al. has shown that PEGylated gold nanoparticles of $75 \pm 25 \mathrm{~nm}$ in diameter target the mesangium of kidneys ${ }^{70}$.

After the second injection, 2.6 $\pm 0.7,2.0 \pm 0.4,4.2 \pm 0.6,4.7 \pm 0.8 \%$ of the I.D. accumulated in the spleens of mice of FF, SF, SS and FS groups, respectively. On the other hand $27.0 \pm 9.7$, $21.3 \pm 6.1,40.7 \pm 3.4$, and $54.6 \pm 14.1 \%$ of the I.D. were found in livers of mice of the FF, SF, SS and FS groups, whereas, $7.1 \pm 6.8,5.0 \pm 0.8,8.6 \pm 2.2$, and $12.3 \pm 2.0 \%$ of the I.D. were detected in kidneys, respectively. The micelles accumulation in brain, heart, and lungs was negligible. The significant difference in the accumulation of star- and flower-like micelles in the liver and spleen coincides with blood circulation kinetics. Concentration of micelles in blood circulation $24 \mathrm{~h}$ after the second injection in FS and SS groups were $4 \%$ of I.D. and below detection limit, respectively. This confirms that the liver and spleen were actively involved in the clearance of star-like micelles after the second injection.

The part of the organs that was not used for quantification of micelles was sectioned and subsequently stained for nuclei and actin (SI-Fig. 9 and 10). The highest fluorescence was 
detected in livers where Kupffer cells are known to play an important role in the clearance of nanoparticles ${ }^{71,72}$.
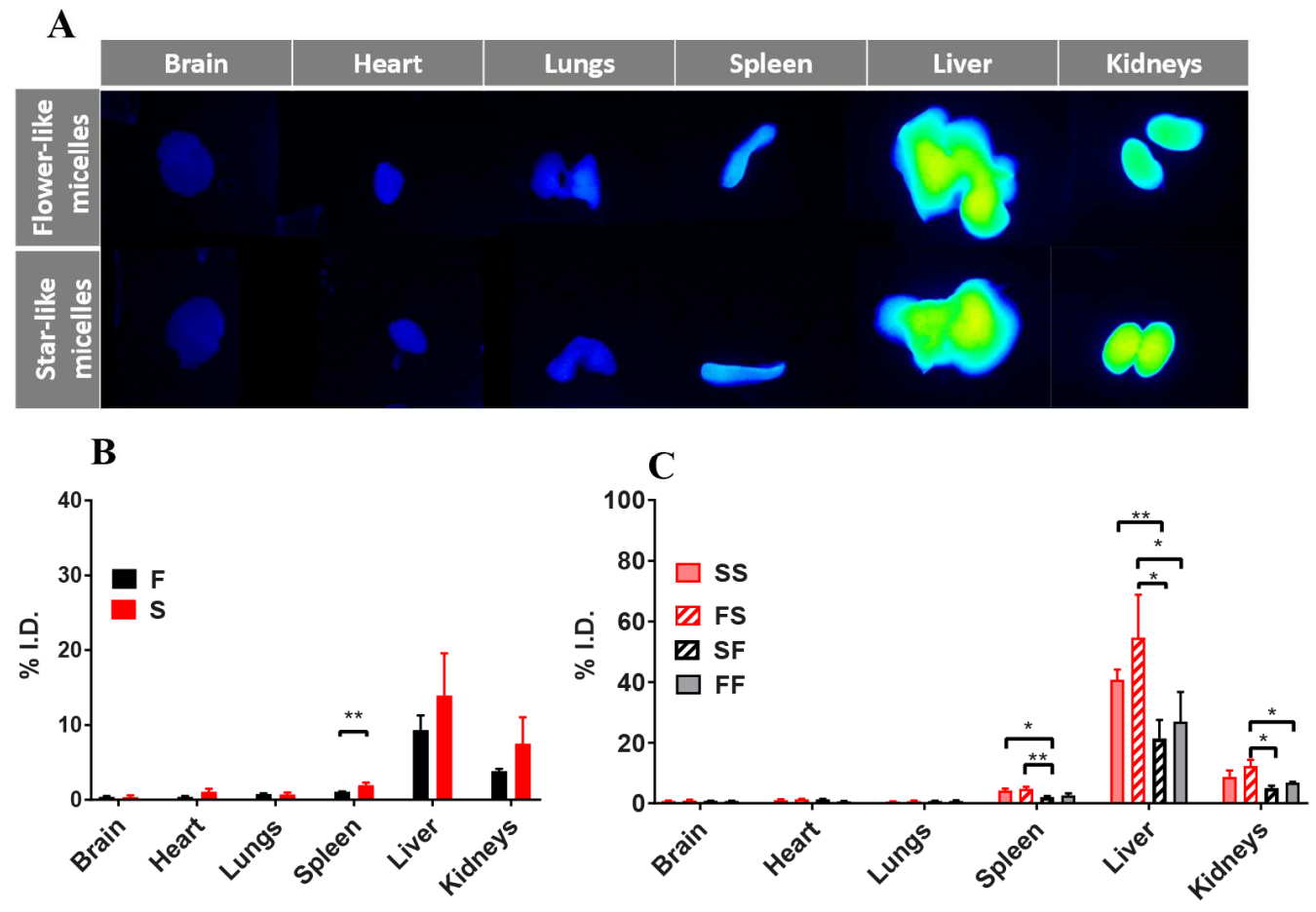

Figure 4: In vivo distribution of Alexa 750-labeled micelles in the major organs (brain, heart, lungs, spleen, liver, and kidneys) of immunocompetent BALB/c mice. Micelles were injected intravenously at a concentration of $15 \mathrm{mg}^{\mathrm{mL}} \mathrm{m}^{-1}(100 \mu \mathrm{L})$ and mice were sacrificed $24 \mathrm{~h}$ after administration. A) Fluorescence image of excised tissues after the first injection taken by LI-COR Pearl ${ }^{\circledR}$ impulse imager. $B, C)$ Quantification of micelle biodistributions in mice by measuring the fluorescence intensity of homogenized tissues that were collected $24 \mathrm{~h}$ after the first $(\mathrm{B})$ and the second injection (C). ${ }^{*} \mathrm{p}<$ $0.05, * * \mathrm{p}<0.01$.

\section{Conclusion}

This study demonstrates that, first, flower-like micelles circulate significantly longer than star-like micelles upon the first injection. Second, both types of micelles induce production of anti-PEG IgM. Third, the anti-PEG IgM induced by star-like micelles causes rapid clearance of a second dose of star-like micelles but not of flower-like micelles. Fourth, the anti-PEG 
IgM induced upon injection of flower-like micelles reduced the circulation time of the second dose of star-like micelles but not of flower-like micelles. Therefore, regardless of the presence of anti-PEG antibodies, flower-like micelles showed similar circulation kinetics after the first and the second injection. In contrast, circulation kinetics of star-like micelles was affected by the presence of anti-PEG IgM and circulation kinetics was dramatically shorter after the second injections compared to the first injection. However, better understanding of these observations needs mechanistic studies and bigger animal groups. Taken together, the results of this study show the great potential of looped PEG in preventing recognition by anti-PEG antibodies and thereby opsonization and clearance of PEGylated nanocarriers. This finding proposes that for clinical applications of pegylated nanoparticles especially the ones that are repeatedly administered, surface decoration with looped PEG should be considered.

\section{Acknowledgments}

The Netherlands Organization for Scientific Research (NWO/VIDI 13457 and NWO/Aspasia 015.009.038) is acknowledged for funding. 


\section{References}

1. Oerlemans, C.; Bult, W.; Bos, M.; Storm, G.; Nijsen, J. F. W.; Hennink, W. E., Polymeric Micelles in Anticancer Therapy: Targeting, Imaging and Triggered Release. Pharmaceutical Research 2010, 27 (12), 2569-2589.

2. Cabral, H.; Kataoka, K., Progress of drug-loaded polymeric micelles into clinical studies. Journal of Controlled Release 2014, 190, 465-476.

3. Deng, C.; Jiang, Y.; Cheng, R.; Meng, F.; Zhong, Z., Biodegradable polymeric micelles for targeted and controlled anticancer drug delivery: Promises, progress and prospects. Nano Today 2012, 7 (5), 467-480.

4. Varela-Moreira, A.; Shi, Y.; Fens, M. H. A. M.; Lammers, T.; Hennink, W. E.; Schiffelers, R. M., Clinical application of polymeric micelles for the treatment of cancer. Materials Chemistry Frontiers 2017, 1 (8), 1485-1501.

5. Talelli, M.; Barz, M.; Rijcken, C. J. F.; Kiessling, F.; Hennink, W. E.; Lammers, T., Core-crosslinked polymeric micelles: Principles, preparation, biomedical applications and clinical translation. Nano Today 2015, 10 (1), 93-117.

6. Torchilin, V. P., Micellar nanocarriers: pharmaceutical perspectives. Pharmaceutical Research 2007, 24 (1), 1-16.

7. Butcher, N. J.; Mortimer, G. M.; Minchin, R. F., Unravelling the stealth effect. Nature Nanotechnology 2016, 11, 310-311.

8. Harris, J. M.; Chess, R. B., Effect of pegylation on pharmaceuticals. Nature Reviews Drug Discovery 2003, 2 (3), 214-221.

9. Schöttler, S.; Becker, G.; Winzen, S.; Steinbach, T.; Mohr, K.; Landfester, K.; Mailänder, V.; Wurm, F. R., Protein adsorption is required for stealth effect of poly(ethylene glycol)- and poly(phosphoester)-coated nanocarriers. Nature Nanotechnology 2016, 11 (4), 372-377.

10. Miceli, E.; Kar, M.; Calderón, M., Interactions of organic nanoparticles with proteins in physiological conditions. Journal of Materials Chemistry B 2017, 5 (23), 4393-4405.

11. Dai, Q.; Bertleff-Zieschang, N.; Braunger, J. A.; Björnmalm, M.; Cortez-Jugo, C.; Caruso, F., Particle Targeting in Complex Biological Media. Advanced Healthcare Materials 2018, 7 (1), 1700575-1700607.

12. Lankveld, D. P. K.; Rayavarapu, R. G.; Krystek, P.; Oomen, A. G.; Verharen, H. W.; van Leeuwen, T. G.; De Jong, W. H.; Manohar, S., Blood clearance and tissue distribution of PEGylated and non-PEGylated gold nanorods after intravenous administration in rats. Nanomedicine 2011, 6 (2), 339-349. 
13. Peracchia, M. T.; Fattal, E.; Desmaële, D.; Besnard, M.; Noël, J. P.; Gomis, J. M.; Appel, M.; d'Angelo, J.; Couvreur, P., Stealth ${ }^{\circledR}$ PEGylated polycyanoacrylate nanoparticles for intravenous administration and splenic targeting. Journal of Controlled Release 1999, 60 (1), 121-128.

14. Yoo, J.-W.; Chambers, E.; Mitragotri, S., Factors that Control the Circulation Time of Nanoparticles in Blood: Challenges, Solutions and Future Prospects. Current Pharmaceutical Design 2010, 16 (21), 2298-2307.

15. Zhou, H.; Fan, Z.; Li, P. Y.; Deng, J.; Arhontoulis, D. C.; Li, C. Y.; Bowne, W. B.; Cheng, H., Dense and Dynamic Polyethylene Glycol Shells Cloak Nanoparticles from Uptake by Liver Endothelial Cells for Long Blood Circulation. ACS Nano 2018, 12 (10), 1013010141.

16. Ishida, T.; Kiwada, H., Anti-polyethyleneglycol Antibody Response to PEGylated Substances. Biological and Pharmaceutical Bulletin 2013, 36 (6), 889-891.

17. Verhoef, J. J. F.; Carpenter, J. F.; Anchordoquy, T. J.; Schellekens, H., Potential induction of anti-PEG antibodies and complement activation toward PEGylated therapeutics. Drug Discovery Today 2014, 19 (12), 1945-1952.

18. Dams, E. T. M.; Laverman, P.; Oyen, W. J. G.; Storm, G.; Scherphof, G. L.; van der Meer, J. W. M.; Corstens, F. H. M.; Boerman, O. C., Accelerated Blood Clearance and Altered Biodistribution of Repeated Injections of Sterically Stabilized Liposomes. Journal of Pharmacology and Experimental Therapeutics 2000, 292 (3), 1071-1079.

19. Koide, H.; Asai, T.; Hatanaka, K.; Akai, S.; Ishii, T.; Kenjo, E.; Ishida, T.; Kiwada, H.; Tsukada, H.; Oku, N., T cell-independent B cell response is responsible for ABC phenomenon induced by repeated injection of PEGylated liposomes. International Journal of Pharmaceutics 2010, 392 (1), 218-223.

20. Ishida, T.; Maeda, R.; Ichihara, M.; Irimura, K.; Kiwada, H., Accelerated clearance of PEGylated liposomes in rats after repeated injections. Journal of Controlled Release 2003, 88 (1), 35-42.

21. Wang, X.; Ishida, T.; Kiwada, H., Anti-PEG IgM elicited by injection of liposomes is involved in the enhanced blood clearance of a subsequent dose of PEGylated liposomes. Journal of Controlled Release 2007, 119 (2), 236-244.

22. Abu Lila, A. S.; Kiwada, H.; Ishida, T., The accelerated blood clearance (ABC) phenomenon: Clinical challenge and approaches to manage. Journal of Controlled Release 2013, 172 (1), 38-47.

23. Koide, H.; Asai, T.; Hatanaka, K.; Urakami, T.; Ishii, T.; Kenjo, E.; Nishihara, M.; Yokoyama, M.; Ishida, T.; Kiwada, H.; Oku, N., Particle size-dependent triggering of 
accelerated blood clearance phenomenon. International Journal of Pharmaceutics 2008, 362 (1), 197-200.

24. Ishida, T.; Harada, M.; Wang, X. Y.; Ichihara, M.; Irimura, K.; Kiwada, H., Accelerated blood clearance of PEGylated liposomes following preceding liposome injection: Effects of lipid dose and PEG surface-density and chain length of the first-dose liposomes. Journal of Controlled Release 2005, 105 (3), 305-317.

25. Sherman, M. R.; Williams, L. D.; Sobczyk, M. A.; Michaels, S. J.; Saifer, M. G. P., Role of the Methoxy Group in Immune Responses to mPEG-Protein Conjugates. Bioconjugate Chemistry 2012, 23 (3), 485-499.

26. Shimizu, T.; Abu Lila, A. S.; Fujita, R.; Awata, M.; Kawanishi, M.; Hashimoto, Y.; Okuhira, K.; Ishima, Y.; Ishida, T., A hydroxyl PEG version of PEGylated liposomes and its impact on anti-PEG IgM induction and on the accelerated clearance of PEGylated liposomes. European Journal of Pharmaceutics and Biopharmaceutics 2018, 127, 142-149.

27. Ishida, T.; Ichikawa, T.; Ichihara, M.; Sadzuka, Y.; Kiwada, H., Effect of the physicochemical properties of initially injected liposomes on the clearance of subsequently injected PEGylated liposomes in mice. Journal of Controlled Release 2004, 95 (3), 403-412.

28. Suzuki, T.; Ichihara, M.; Hyodo, K.; Yamamoto, E.; Ishida, T.; Kiwada, H.; Ishihara, H.; Kikuchi, H., Accelerated blood clearance of PEGylated liposomes containing doxorubicin upon repeated administration to dogs. International Journal of Pharmaceutics 2012, 436 (1), 636-643.

29. Suzuki, T.; Ichihara, M.; Hyodo, K.; Yamamoto, E.; Ishida, T.; Kiwada, H.; Kikuchi, H.; Ishihara, H., Influence of dose and animal species on accelerated blood clearance of PEGylated liposomal doxorubicin. International Journal of Pharmaceutics 2014, 476 (1), 205-212.

30. Garay, R. P.; El-Gewely, R.; Armstrong, J. K.; Garratty, G.; Richette, P., Antibodies against polyethylene glycol in healthy subjects and in patients treated with PEG-conjugated agents. Expert Opinion on Drug Delivery 2012, 9 (11), 1319-1323.

31. Armstrong, J. K., The occurrence, induction, specificity and potential effect of antibodies against poly(ethylene glycol). In PEGylated Protein Drugs: Basic Science and Clinical Applications, Veronese, F. M., Ed. Birkhäuser Basel: Basel, 2009; pp 147-168.

32. Le, Y.; Toyofuku, W. M.; Scott, M. D., Immunogenicity of murine mPEG-red blood cells and the risk of anti-PEG antibodies in human blood donors. Experimental Hematology 2017, 47, 36-47.

33. Kloos, R. Q. H. v. d. S., I. M.; Mastrobattista, E.; Hennink, W. E.; Pieters, R.; Verhoef, J. J. F., Acute Lymphoblastic Leukaemia Patients Treated with PEGasparaginase Develop Antibodies to PEG and the Succinate Linker. In Press 2019. 
34. Armstrong, J. K.; Hempel, G.; Koling, S.; Chan, L. S.; Fisher, T.; Meiselman, H. J.; Garratty, G., Antibody against poly(ethylene glycol) adversely affects PEG-asparaginase therapy in acute lymphoblastic leukemia patients. Cancer 2007, 110 (1), 103-111.

35. Walkey, C. D.; Olsen, J. B.; Guo, H.; Emili, A.; Chan, W. C. W., Nanoparticle Size and Surface Chemistry Determine Serum Protein Adsorption and Macrophage Uptake. Journal of the American Chemical Society 2012, 134 (4), 2139-2147.

36. Perry, J. L.; Reuter, K. G.; Kai, M. P.; Herlihy, K. P.; Jones, S. W.; Luft, J. C.; Napier, M.; Bear, J. E.; DeSimone, J. M., PEGylated PRINT Nanoparticles: The Impact of PEG Density on Protein Binding, Macrophage Association, Biodistribution, and Pharmacokinetics. Nano Letters 2012, 12 (10), 5304-5310.

37. Unsworth, L. D.; Sheardown, H.; Brash, J. L., Protein-Resistant Poly(ethylene oxide)Grafted Surfaces: Chain Density-Dependent Multiple Mechanisms of Action. Langmuir 2008, 24 (5), 1924-1929.

38. Satulovsky, J.; Carignano, M. A.; Szleifer, I., Kinetic and thermodynamic control of protein adsorption. Proceedings of the National Academy of Sciences 2000, 97 (16), 90379041.

39. de Graaf, A. J.; Boere, K. W.; Kemmink, J.; Fokkink, R. G.; van Nostrum, C. F.; Rijkers, D. T.; van der Gucht, J.; Wienk, H.; Baldus, M.; Mastrobattista, E.; Vermonden, T.; Hennink, W. E., Looped structure of flowerlike micelles revealed by $1 \mathrm{H}$ NMR relaxometry and light scattering. Langmuir 2011, 27 (16), 9843-9848.

40. Najafi, M.; Kordalivand, N.; Moradi, M. A.; van den Dikkenberg, J.; Fokkink, R.; Friedrich, H.; Sommerdijk, N.; Hembury, M.; Vermonden, T., Native Chemical Ligation for Cross-Linking of Flower-Like Micelles. Biomacromolecules 2018, 19 (9), 3766-3775.

41. Ulbrich, K.; Šubr, V.; Strohalm, J.; Plocová, D.; Jelínková, M.; Říhová, B., Polymeric drugs based on conjugates of synthetic and natural macromolecules: I. Synthesis and physico-chemical characterisation. Journal of Controlled Release 2000, 64 (1), 63-79.

42. Boere, K. W. M.; Soliman, B. G.; Rijkers, D. T. S.; Hennink, W. E.; Vermonden, T., Thermoresponsive Injectable Hydrogels Cross-Linked by Native Chemical Ligation. Macromolecules 2014, 47 (7), 2430-2438.

43. Boere, K. W.; van den Dikkenberg, J.; Gao, Y.; Visser, J.; Hennink, W. E.; Vermonden, T., Thermogelling and Chemoselectively Cross-Linked Hydrogels with Controlled Mechanical Properties and Degradation Behavior. Biomacromolecules 2015, 16 (9), 2840-51.

44. Soga, O.; van Nostrum, C. F.; Ramzi, A.; Visser, T.; Soulimani, F.; Frederik, P. M.; Bomans, P. H. H.; Hennink, W. E., Physicochemical Characterization of Degradable Thermosensitive Polymeric Micelles. Langmuir 2004, 20 (21), 9388-9395. 
45. Matyjaszewski, K.; Xia, J., Atom Transfer Radical Polymerization. Chemical Reviews 2001, 101 (9), 2921-2990.

46. Trzebicka, B.; Robak, B.; Trzcinska, R.; Szweda, D.; Suder, P.; Silberring, J.; Dworak, A., Thermosensitive PNIPAM-peptide conjugate - Synthesis and aggregation. European Polymer Journal 2013, 49 (2), 499-509.

47. Ganachaud, F.; Monteiro, M. J.; Gilbert, R. G.; Dourges, M.-A.; Thang, S. H.; Rizzardo, E., Molecular Weight Characterization of Poly(N-isopropylacrylamide) Prepared by Living Free-Radical Polymerization. Macromolecules 2000, 33 (18), 6738-6745.

48. Smithenry, D. W.; Kang, M.-S.; Gupta, V. K., Telechelic Poly(N-isopropylacrylamide): Polymerization and Chain Aggregation in Solution. Macromolecules 2001, 34 (24), 85038511.

49. Dawson, P. E.; Muir, T. W.; Clark-Lewis, I.; Kent, S. B., Synthesis of proteins by native chemical ligation. Science 1994, 266 (5186), 776-779.

50. Najafi, M.; Hebels, E.; Hennink, W. E.; Vermonden, T., Poly( N-isopropylacrylamide): Physicochemical Properties and Biomedical Applications: Chemistry, Properties and Applications. In Temperature-Responsive Polymers: Chemistry, Properties, and Applications, Khutoryanskiy, V. V.; Georgiou, K. T., Eds. John Wiley \& Sons: 2018; pp 1-34.

51. Talelli, M.; Iman, M.; Varkouhi, A. K.; Rijcken, C. J. F.; Schiffelers, R. M.; Etrych, T.; Ulbrich, K.; van Nostrum, C. F.; Lammers, T.; Storm, G.; Hennink, W. E., Core-crosslinked polymeric micelles with controlled release of covalently entrapped doxorubicin. Biomaterials 2010, 31 (30), 7797-7804.

52. aharoni, S. M.; Murthy, N. S., Experimental observation of the onset of finite domain boundaries in a simple two-phase system by small-angle $\mathrm{x}$-ray scattering. Macromolecules 1983, 16, 1943-1944.

53. van de Sande, W.; Persoons, A., The size and shape of macromolecular structures: determination of the radius, the length and the persistance length of rod-like micelles of dodecyldimethylammonium chloride and bromide. Journal of Physical Chemistry 1985, 89 (3), 404-406.

54. Martínez-Jothar, L.; Doulkeridou, S.; Schiffelers, R. M.; Sastre Torano, J.; Oliveira, S.; van Nostrum, C. F.; Hennink, W. E., Insights into maleimide-thiol conjugation chemistry: Conditions for efficient surface functionalization of nanoparticles for receptor targeting. Journal of Controlled Release 2018, 282, 101-109.

55. Lowe, A. B., Thiol-ene "click" reactions and recent applications in polymer and materials synthesis. Polymer Chemistry 2010, 1 (1), 17-36. 
56. Ichihara, M.; Shimizu, T.; Imoto, A.; Hashiguchi, Y.; Uehara, Y.; Ishida, T.; Kiwada, H., Anti-PEG IgM Response against PEGylated Liposomes in Mice and Rats. Pharmaceutics 2011, 3 (1), 1-11.

57. Ishida, T.; Masuda, K.; Ichikawa, T.; Ichihara, M.; Irimura, K.; Kiwada, H., Accelerated clearance of a second injection of PEGylated liposomes in mice. International Journal of Pharmaceutics 2003, 255 (1), 167-174.

58. Zhang, Y.; Huo, M.; Zhou, J.; Xie, S., PKSolver: An add-in program for pharmacokinetic and pharmacodynamic data analysis in Microsoft Excel. Computer Methods and Programs in Biomedicine 2010, 99 (3), 306-314.

59. Yue, J.; Liu, S.; Xie, Z.; Xing, Y.; Jing, X., Size-dependent biodistribution and antitumor efficacy of polymer micelle drug delivery systems. Journal of Materials Chemistry B 2013, 1 (34), 4273-4280.

60. Cabral, H.; Matsumoto, Y.; Mizuno, K.; Chen, Q.; Murakami, M.; Kimura, M.;Terada, Y.; Kano, M. R.; Miyazono, K.; Uesaka, M.; Nishiyama, N.; Kataoka, K., Accumulation of sub-100 $\mathrm{nm}$ polymeric micelles in poorly permeable tumours depends on size. Nature Nanotechnology 2011, 6 (12), 815-823.

61. Li, M.; Al-Jamal, K. T.; Kostarelos, K.; Reineke, J., Physiologically Based Pharmacokinetic Modeling of Nanoparticles. ACS Nano 2010, 4 (11), 6303-6317.

62. Yang, X.; Chen, Q.; Yang, J.; Wu, S.; Liu, J.; Li, Z.; Liu, D.; Chen, X.; Qiu, Y., TumorTargeted Accumulation of Ligand-Installed Polymeric Micelles Influenced by Surface PEGylation Crowdedness. ACS Applied Materials \& Interfaces 2017, 9 (50), 44045-44052.

63. Du, X.-J.; Wang, J.-L.; Liu, W.-W.; Yang, J.-X.; Sun, C.-Y.; Sun, R.; Li, H.-J.; Shen, S.; Luo, Y.-L.; Ye, X.-D.; Zhu, Y.-H.; Yang, X.-Z.; Wang, J., Regulating the surface poly(ethylene glycol) density of polymeric nanoparticles and evaluating its role in drug delivery in vivo. Biomaterials 2015, 69, 1-11.

64. Li, S.-D.; Huang, L., Pharmacokinetics and Biodistribution of Nanoparticles. Molecular Pharmaceutics 2008, 5 (4), 496-504.

65. Qin, L.; Smith, B. D.; Tsai, H. L.; Yaghi, N. K.; Neela, P. H.; Moake, M.; Fu, J.; Kasamon, Y. L.; Prince, G. T.; Goswami, M.; Rosner, G. L.; Levitsky, H. I.; Hourigan, C. S., Induction of high-titer IgG antibodies against multiple leukemia-associated antigens in CML patients with clinical responses to K562/GVAX immunotherapy. Blood Cancer Journal 2013, 3 (9), e145 (111).

66. Jackson, K. J. L.; Wang, Y.; Collins, A. M., Human immunoglobulin classes and subclasses show variability in VDJ gene mutation levels. Immunology and Cell Biology 2014, 92 (8), 729-733. 
67. Rijcken, C. J.; Snel, C. J.; Schiffelers, R. M.; van Nostrum, C. F.; Hennink, W. E., Hydrolysable core-crosslinked thermosensitive polymeric micelles: Synthesis, characterisation and in vivo studies. Biomaterials 2007, 28 (36), 5581-5593.

68. Alexis, F.; Pridgen, E.; Molnar, L. K.; Farokhzad, O. C., Factors Affecting the Clearance and Biodistribution of Polymeric Nanoparticles. Molecular Pharmaceutics 2008, 5 (4), 505-515.

69. Scarfe, L.; Rak-Raszewska, A.; Geraci, S.; Darssan, D.; Sharkey, J.; Huang, J.; Burton, N. C.; Mason, D.; Ranjzad, P.; Kenny, S.; Gretz, N.; Lévy, R.; Kevin Park, B.; García-Fiñana, M.; Woolf, A. S.; Murray, P.; Wilm, B., Measures of kidney function by minimally invasive techniques correlate with histological glomerular damage in SCID mice with adriamycininduced nephropathy. Scientific Reports 2015, 5 (1), 13601-13614.

70. Choi, C. H.; Zuckerman, J. E.; Webster, P.; Davis, M. E., Targeting kidney mesangium by nanoparticles of defined size. Proceedings of the National Academy of Sciences of the United States of America 2011, 108 (16), 6656-6661.

71. Dixon, L. J.; Barnes, M.; Tang, H.; Pritchard, M. T.; Nagy, L. E., Kupffer cells in the liver. Compr Physiol 2013, 3 (2), 785-797.

72. Zhang, Y.-N.; Poon, W.; Tavares, A. J.; McGilvray, I. D.; Chan, W. C. W., Nanoparticleliver interactions: Cellular uptake and hepatobiliary elimination. Journal of Controlled Release 2016, 240, 332-348. 


\section{Supporting Information:}
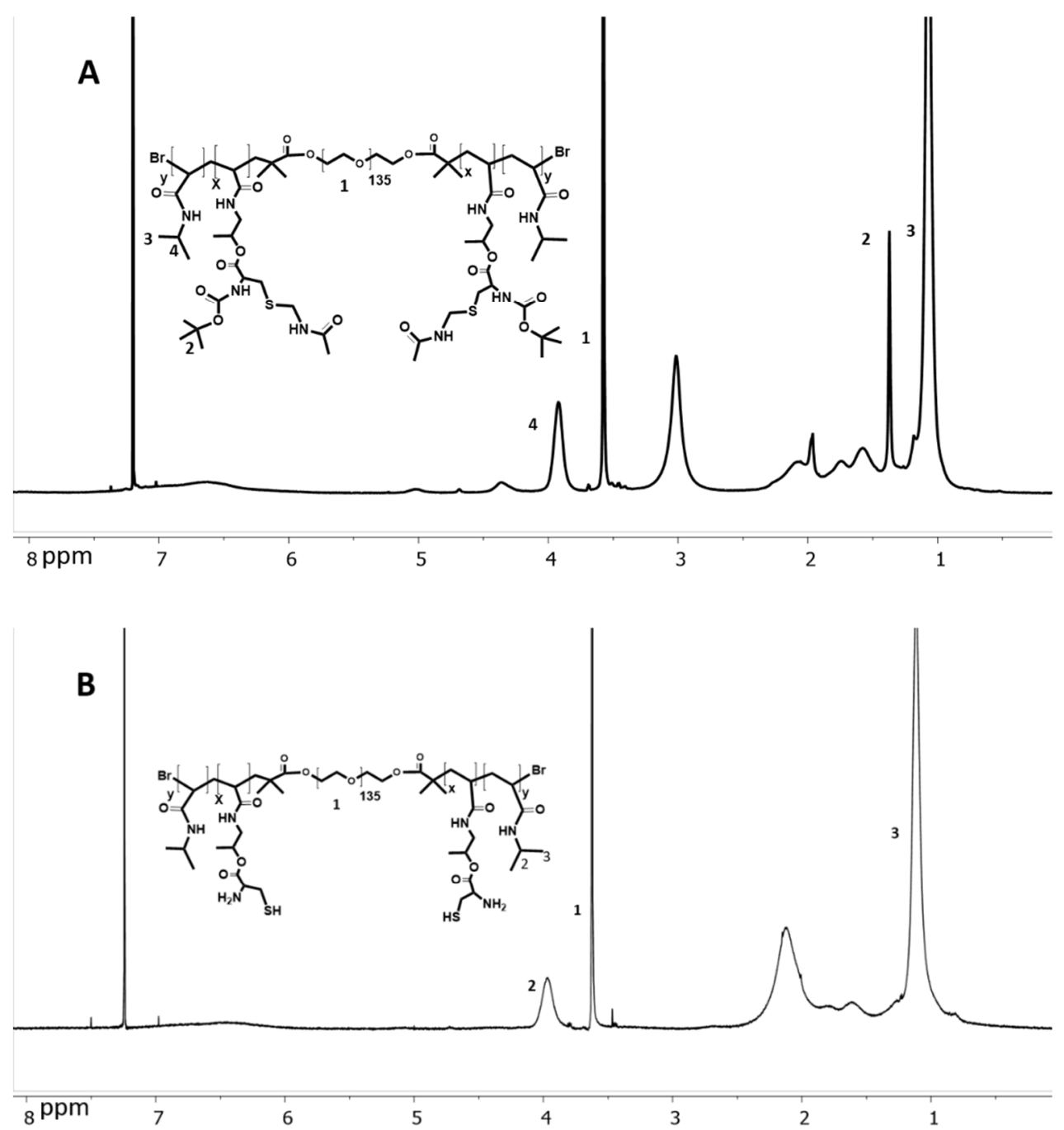

SI-Figure 1: ${ }^{1} \mathrm{H}-\mathrm{NMR}$ spectra of A) protected PNC B) PNC in $\mathrm{CDCl}_{3}$. 


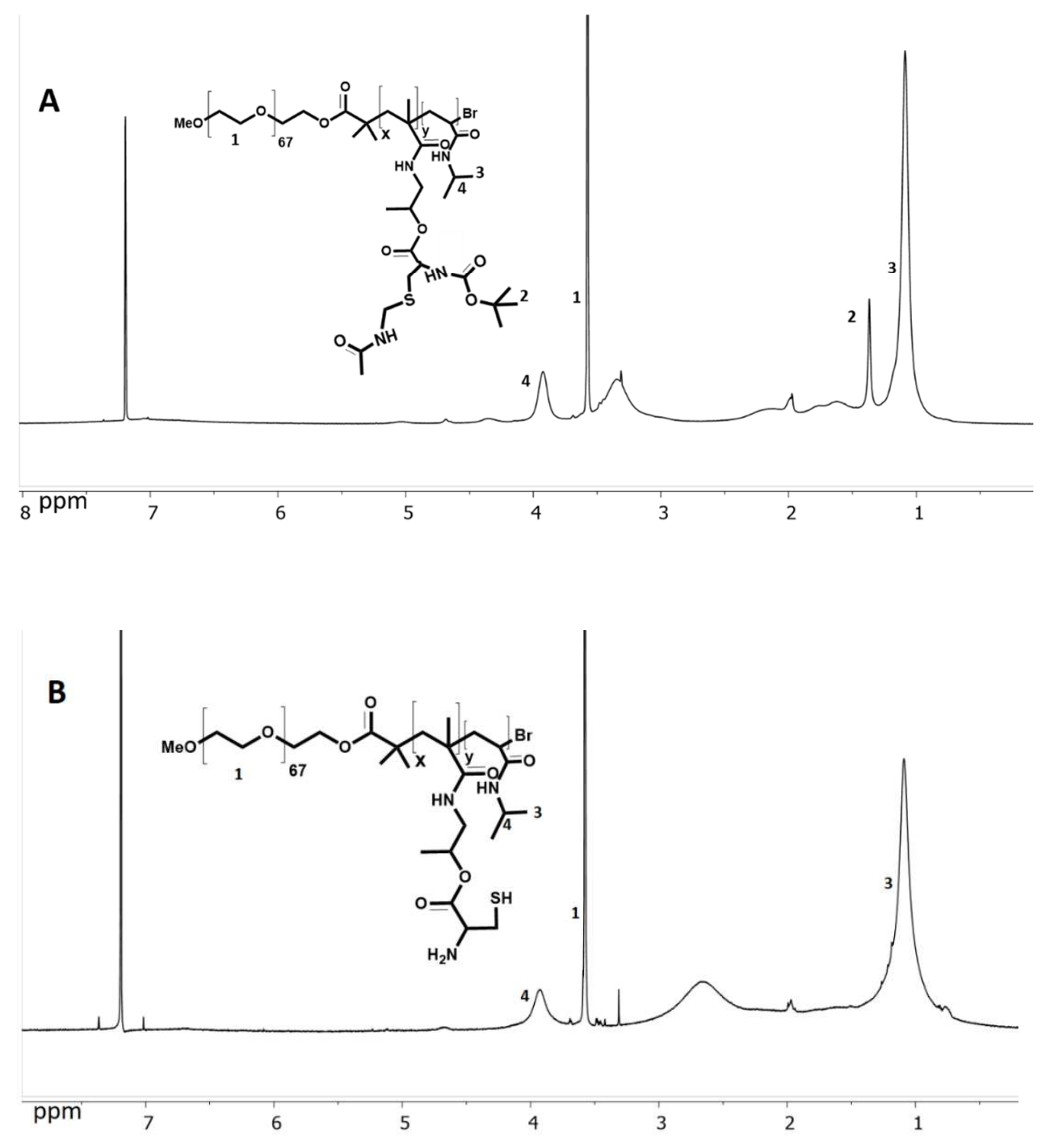

SI-Figure 2: ${ }^{1} \mathrm{H}-\mathrm{NMR}$ spectra of A) protected mPNC, B) mPNC in $\mathrm{CDCl}_{3}$. 


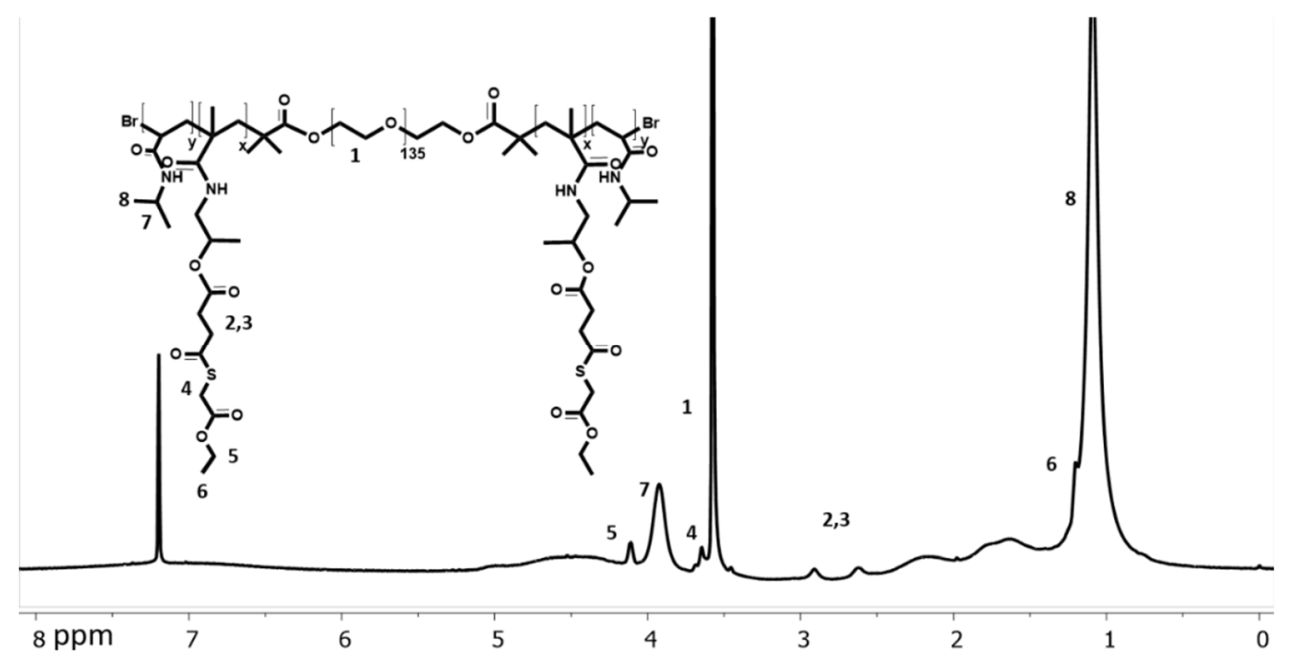

SI-Figure 3: ${ }^{1} \mathrm{H}-\mathrm{NMR}$ spectrum of PNE in $\mathrm{CDCl}_{3}$.

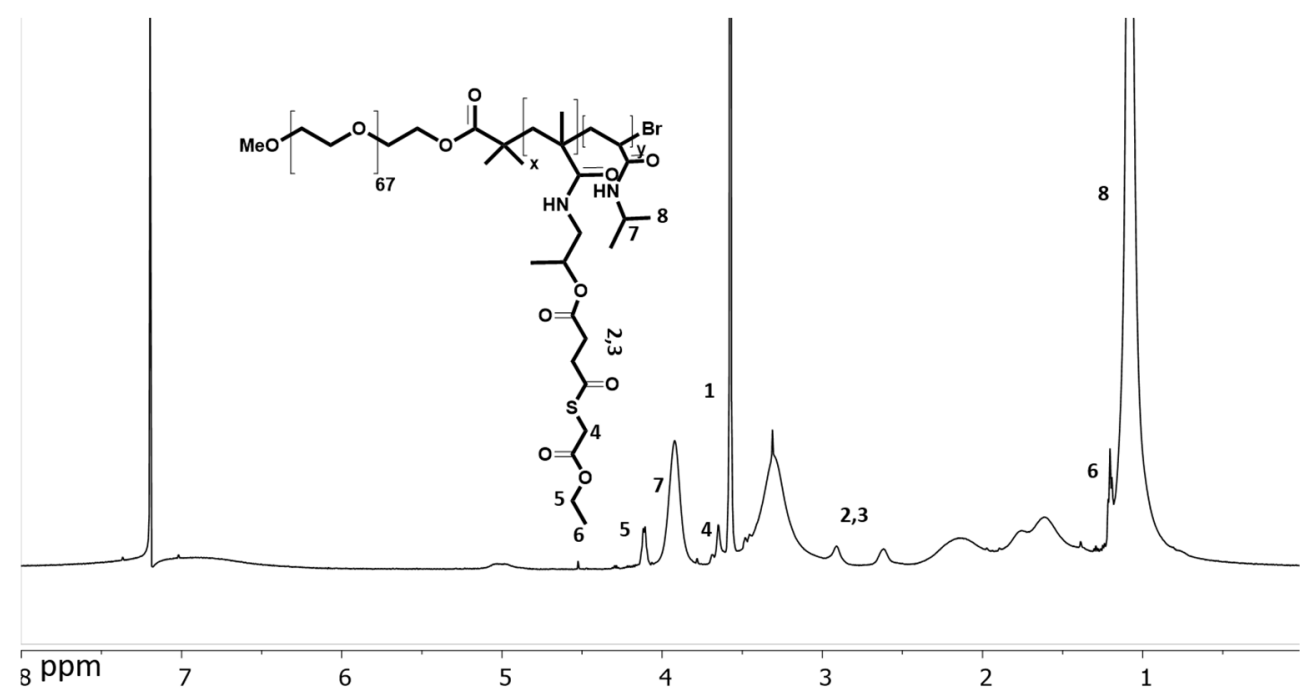

SI-Figure 4: ${ }^{1} \mathrm{H}-\mathrm{NMR}$ spectrum of $\mathrm{mPNE}$ in $\mathrm{CDCl}_{3}$. 

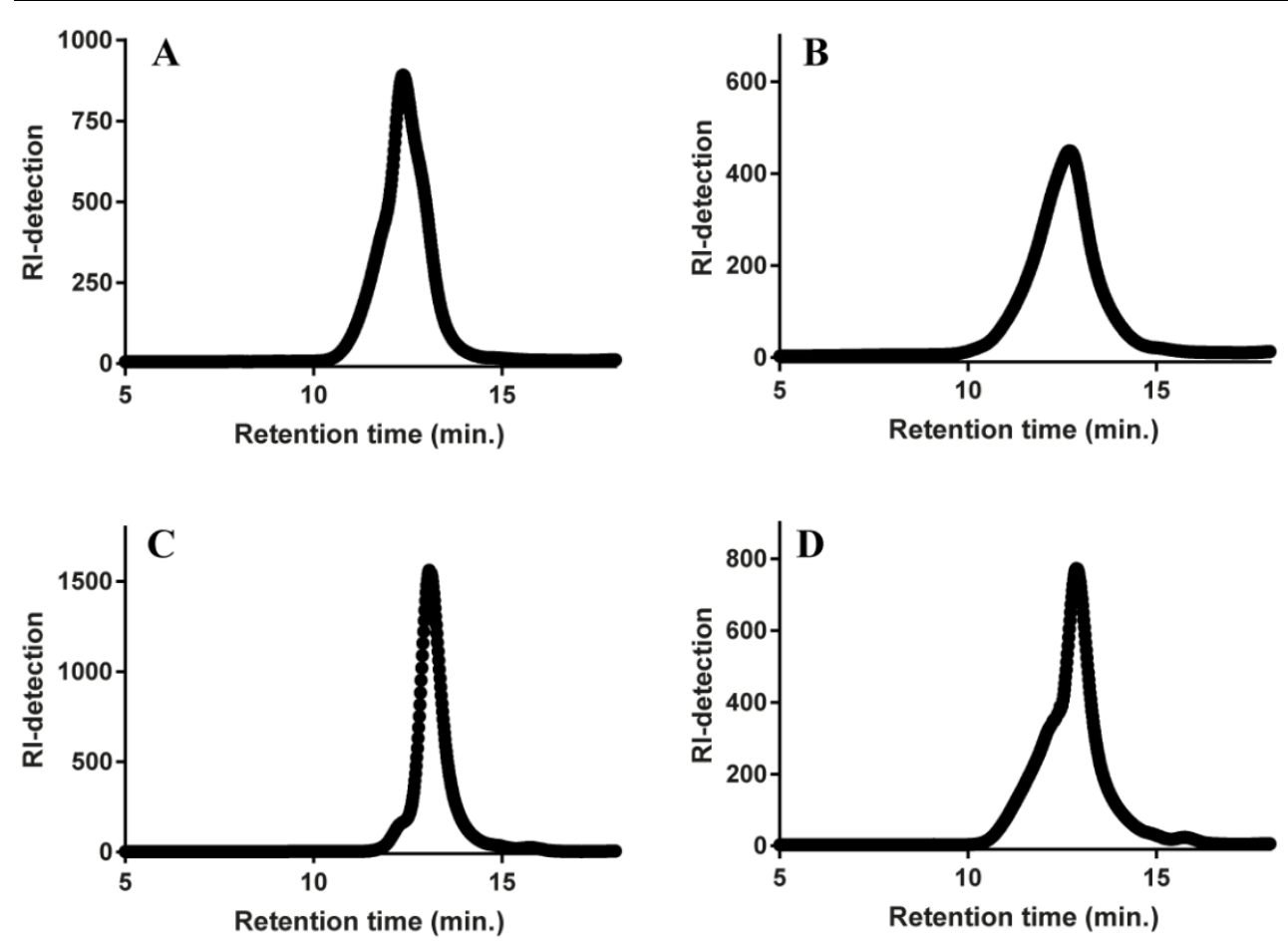

SI-Figure 5: GPC chromatogrammes of A) PNC, B) PNE, C) mPNC, and D) mPNE in DMF containing 10 $\mathrm{mM} \mathrm{LiCl}$.
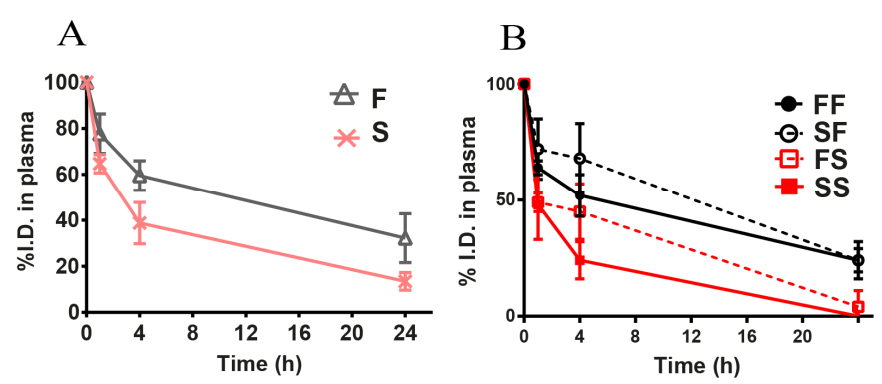

C
\begin{tabular}{|c|c|}
\hline injection & $\mathrm{t}_{1 / 2}(\mathrm{~h})$ \\
\hline $\mathrm{F}$ & $18.6 \pm 3.1$ \\
\hline $\mathrm{FF}$ & $15.4 \pm 2.2$ \\
\hline $\mathrm{SF}$ & $14.4 \pm 1.1$ \\
\hline $\mathrm{S}$ & $10.7 \pm 0.7$ \\
\hline $\mathrm{SS}$ & $2.3 \pm 0.3$ \\
\hline $\mathrm{FS}$ & $7.3 \pm 3.0$ \\
\hline
\end{tabular}

SI-Figure 6: Circulation kinetics of fluorescently labelled (Alexa Fluor ${ }^{\mathrm{TM}}$ 750) flower-like (F) and starlike (S) micelles in immunocompetent BALB/c mice. A) circulation kinetics of flower-like and star-like micelles after the first injection. The presented data are mean values of blood samples of 6 mice. B) circulation kinetics of micelles in mice after the second injection; each data point represents the average of three samples. C) circulation half-life $\left(t_{1} / 2\right)$ calculated based on plasma concentration-time curves of micelles using non-compartmental model by means of PKsolver ${ }^{1}$. 
-.. Only serum $\quad 0 \mathrm{~h} \quad-1 \mathrm{~h} \quad 6 \mathrm{~h} \quad 24 \mathrm{~h}$

A

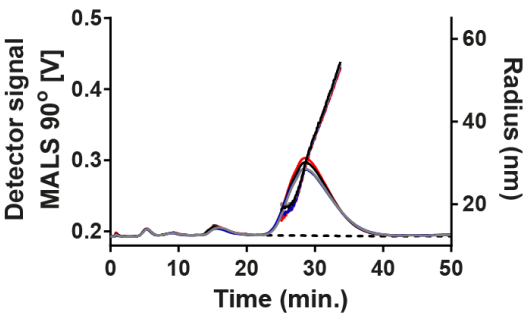

C

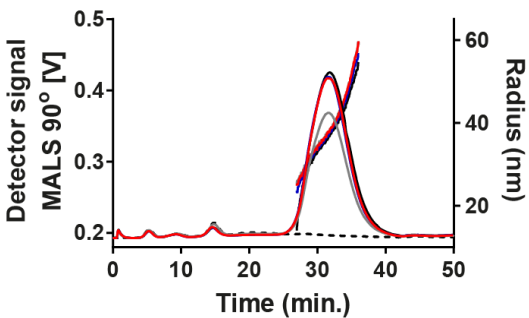

B

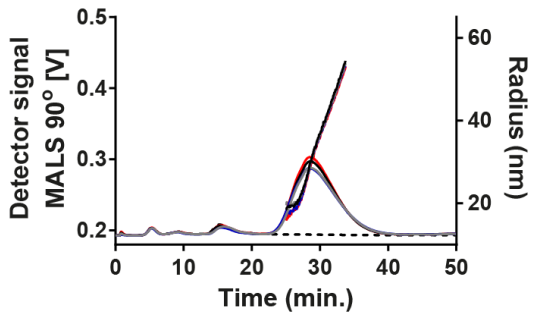

D

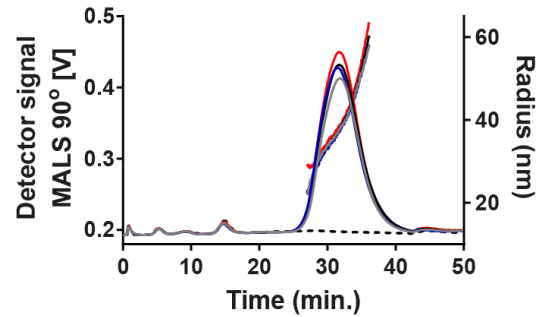

SI-Figure 7: AF4-MALS analysis of micelles upon incubation with human serum either positive for antiPEG IgM and IgG (+ Serum) or negative for both (-Serum). Micelles were diluted 1:1 v/v in plasma to a final concentration of $4.5 \mathrm{mg}^{\mathrm{mL}} \mathrm{m}^{-1}$ and incubated at $37^{\circ} \mathrm{C}$ for $0,1,6$, and $24 \mathrm{~h}$. Serum samples (+ or -) were also diluted 1:1 v/v with PBS prior to the injections. A) flower-like micelles in - serum, B) flower-like micelles in + serum, C) star-like micelles in - serum, and D) star-like micelles in + serum.

SI-table 1: Radius of gyration of micelles $(\mathrm{nm})$ upon incubation with human serum either positive for anti-PEG IgM and IgG (+ Serum) or negative for both (-Serum). Radius of gyration of flower- and starlike micelles in PBS was 28.6 and $35 \mathrm{~nm}$, respectively.

Flower-like micelles

Time (h)

\section{Star-like micelles}

\begin{tabular}{ccccc}
\cline { 2 - 5 } & - serum & + serum & - serum & + serum \\
\hline $\mathbf{0}$ & 30.0 & 29.5 & 35.5 & 34.2 \\
\hline $\mathbf{1}$ & 29.8 & 28.7 & 35.1 & 34.9 \\
\hline $\mathbf{6}$ & 28.7 & 29.5 & 34.9 & 35.3 \\
\hline $\mathbf{2 4}$ & 28.4 & 28.2 & 35.7 & 36.7 \\
\hline
\end{tabular}




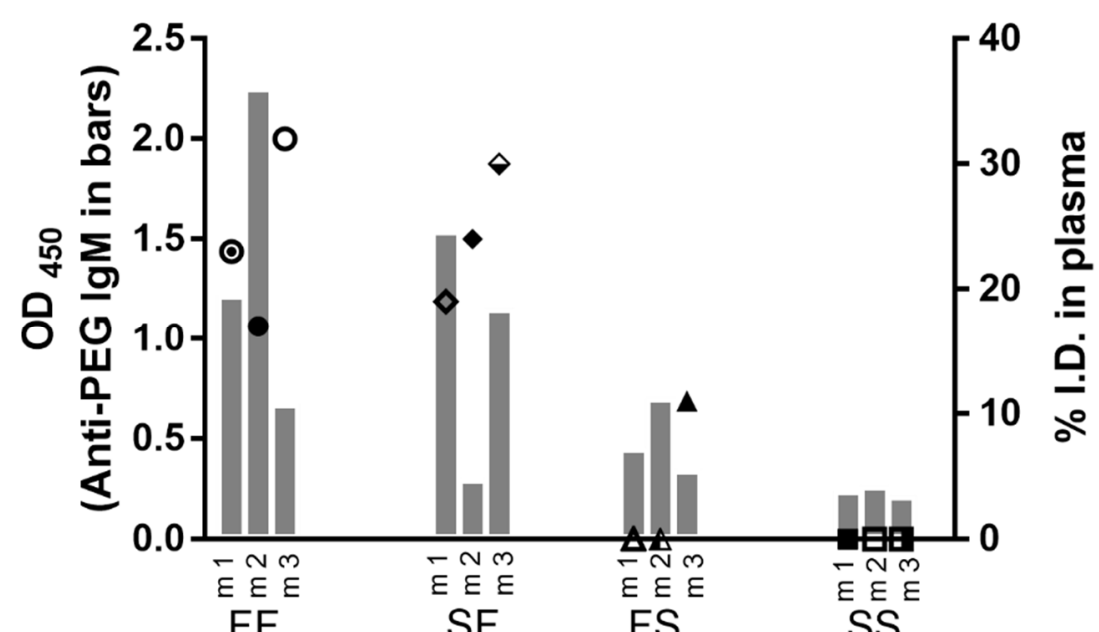

SI-Figure 8: Concentration of anti-PEG IgM in the corresponding blood samples (m1-3 in X-axis refer to mice number). The bars represent the anti-PEG IgM titers and the symbols show \%l.D. in the plasma $24 \mathrm{~h}$ after the second injection. 


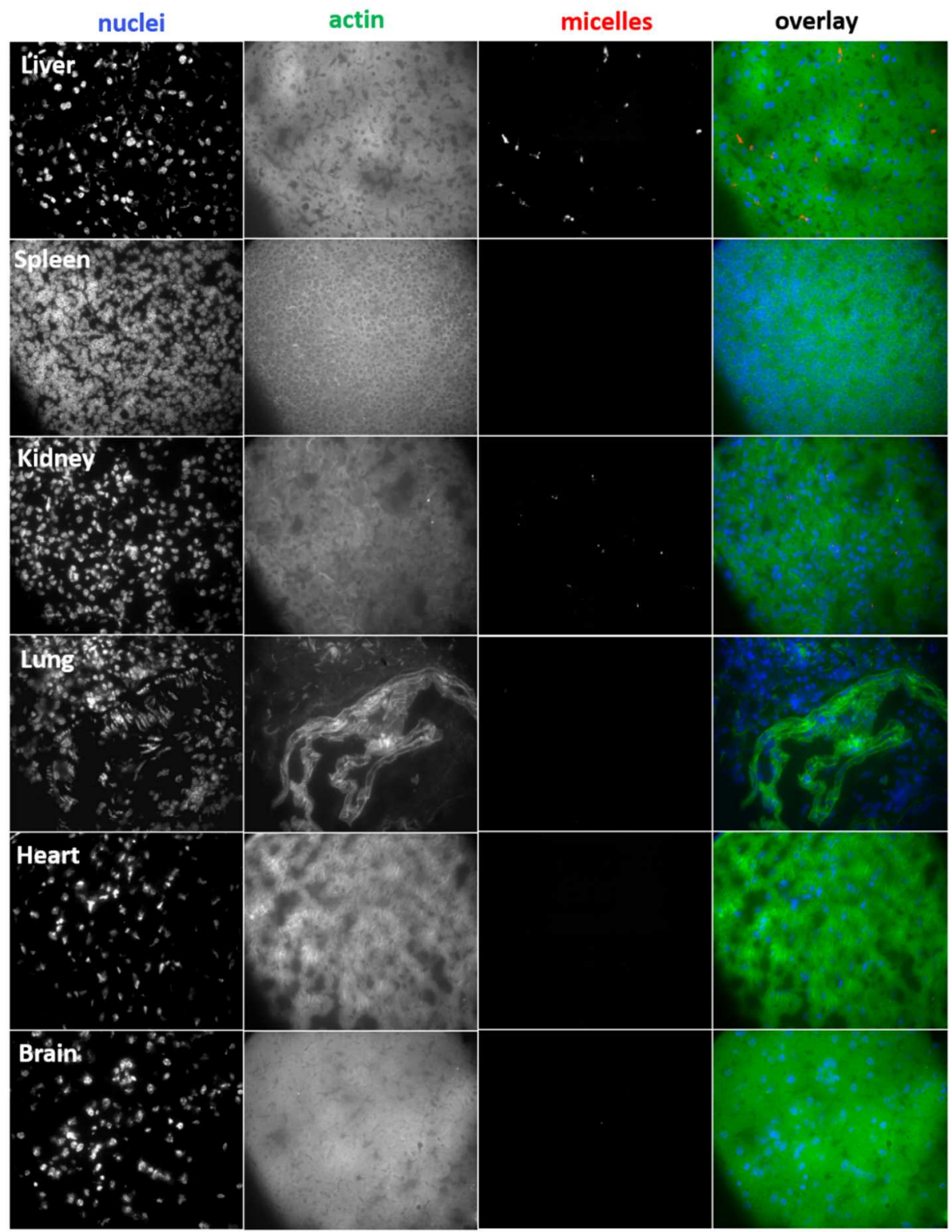

SI-Figure 9: Staining of major organs harvested from mice $24 \mathrm{~h}$ after the first injection of flower-like micelles. The images are taken at $60 \times$ magnification and processed using ImageJ. Nuclei are stained with HOECHST 33342 and depicted in blue, the actin cytoskeleton was stained using PhalloidinAlexa488 and shown in green and Alexa 750-labeled micelles are in red. 


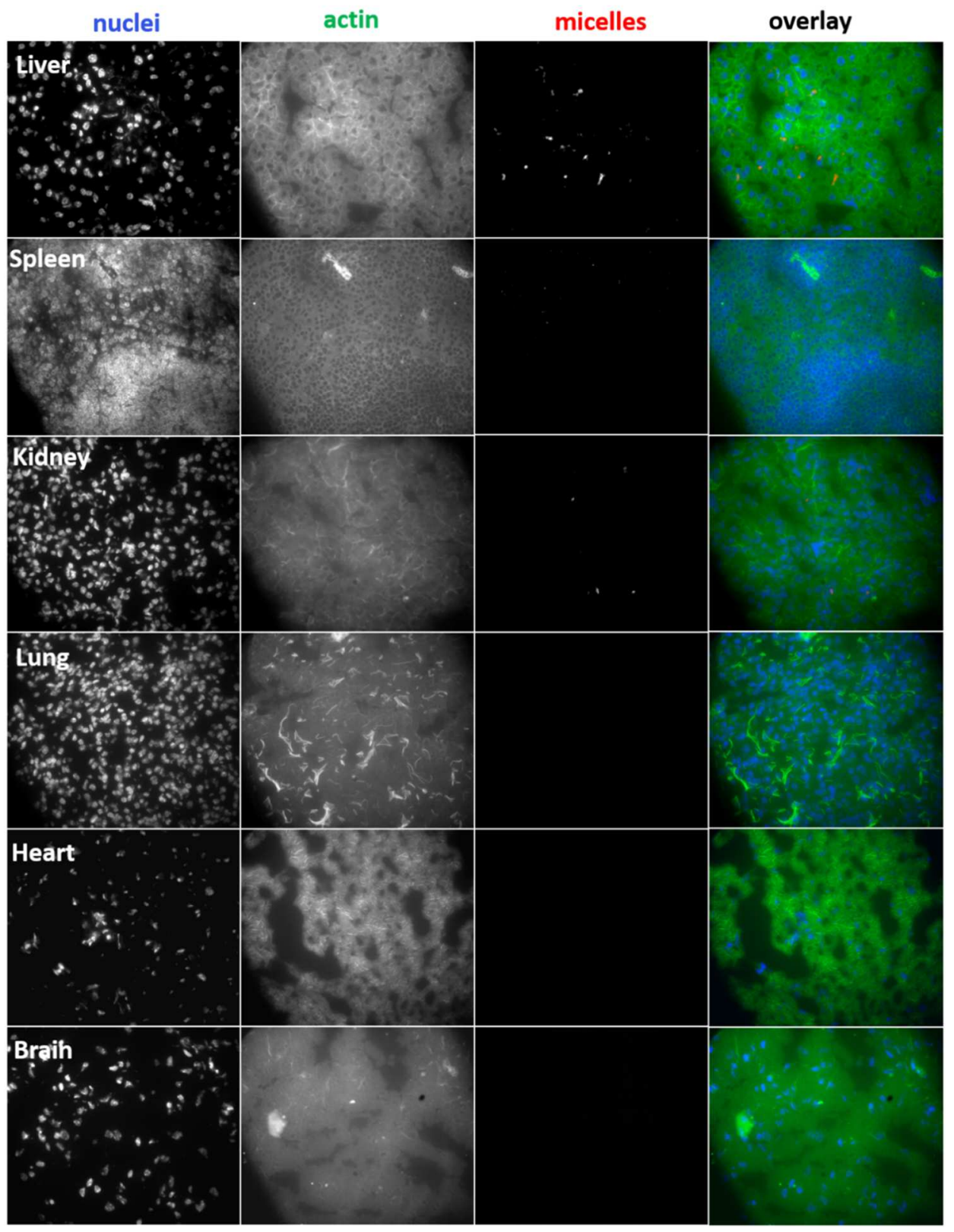

SI-Figure 10: Staining of major organs collected from mice $24 \mathrm{~h}$ after the first injection of star-like micelles. The images are taken at $60 \times$ magnification and processed using ImageJ. Nuclei are stained with HOECHST 33342 and depicted in blue and stained. The actin cytoskeleton stained with PhalloidinAlexa488 and shown in green and Alexa 750-labeled micelles are in red. 


\section{Static light scattering (SLS)}

Both static light scattering (SLS) and dynamic light scattering (DLS) were performed as a function of the detection angle $\Theta$. For SLS the scattering intensities for sample $\left(I_{s}\right)$, solvent (PBS) (Io) and toluene (Itol) as a function of the scattering angle $\Theta$ were measured. Furthermore the dark current Idark (detector intensity with laser switched off) was measured. The obtained intensities were converted into Rayleigh scattering $(R)$ to obtain absolute scattering of the sample using equation 1 :

$$
R(\Theta)=\frac{I_{S}(\Theta)-I_{0}(\Theta)}{I_{\text {tol }}(\Theta)-I_{\text {dark }}} \frac{n_{S}^{2}}{n_{\text {tol }}^{2}} R_{\text {tol }}
$$

In which $n_{t o l}$ is the refractive index of the reference $\left(n_{t o l}=1.494\right), n_{s}$ is the refractive index of the solvent $\left(n_{s}=1.332\right)$ and $R_{t o l}$ is the known absolute Rayleigh scattering for toluene at $632.8 \mathrm{~nm}$ wavelength. For $R_{t o l}$ we used $1.0210^{-3} \mathrm{~m}^{-1}{ }^{2}$; then the scattering angle $\Theta$ was converted into the wave vector $q$ using equation 2 :

$$
q=\frac{4 \pi \mathrm{n}_{s} \sin \frac{\Theta}{2}}{\lambda}
$$

where $\lambda$ is the wavelength in the vacuum.

- $\quad R_{g}$ was obtained according to Guinier ${ }^{3}$ by plotting $\ln (R)$ as a function of $q^{2}$ in which the slope is equal to: $\frac{R_{g}^{2}}{3}$

- $\quad R_{h}$ was obtained by DLS by plotting the decay rate $\Gamma$ extracted from the second order cumulant as a function of $q^{2}$. In case of monodisperse particles this should result in a straight line in which the slope equals to the diffusion coefficient $D$ and from that $R_{h}$ was obtained using equation 3 :

$$
D=\frac{\mathrm{kT}}{6 \pi \eta R_{h}}
$$

- $\quad M_{w}$ was obtained by plotting the absolute scattering intensity $R$ as a function of $q$ and extrapolated to $q=0$. The absolute scattering $R$ should equal to: 
(4)

$$
R=K_{r} C M_{w} \mathrm{~S}(\mathrm{q}) \mathrm{P}(\mathrm{qR})
$$

At low concentration, the structure factor $S(q) \approx 1$ and at $q \rightarrow 0$, the form factor was considered $\mathrm{P}(\mathrm{qR})=1$, therefore, $R(q \rightarrow 0)=K_{r} C, M_{w}$ where $K_{r}$ an optical constant (equation 5 ) and $C$ the particle concentration in $\mathrm{kg} \cdot \mathrm{m}^{-3}$.

$$
K_{r}=\frac{4 n_{S}^{2} \pi^{2}}{\lambda^{4} N_{A v}}\left(\frac{d n}{d c}\right)^{2}
$$

1. Zhang, Y.; Huo, M.; Zhou, J.; Xie, S., PKSolver: An add-in program for pharmacokinetic and pharmacodynamic data analysis in Microsoft Excel. Computer Methods and Programs in Biomedicine 2010, 99 (3), 306-314.

2. $\mathrm{Wu}, \mathrm{H}$., Correlations between the Rayleigh ratio and the wavelength for toluene and benzene. Chemical Physics 2010, 367 (1), 44-47.

3. Guinier, A.; Fournet, G., Small-Angle Scattering of X-Rays. John Wiley and Sons, Inc., New York

1955. 



\section{Chapter 5}

\section{Conversion of an injectable MMP- degradable hydrogel into core- crosslinked micelles}

Marzieh Najafi ${ }^{1}$, Hamed Asadi1,2, Joep van den Dikkenberg ${ }^{1}$, Mies J. van Steenbergen ${ }^{1}$, Marcel H.A.M Fens ${ }^{1}$, Wim E. Hennink ${ }^{1}$, Tina Vermonden ${ }^{1}$

1Department of Pharmaceutics, Utrecht Institute for Pharmaceutical Sciences (UIPS), Science for Life, Faculty of Science, Utrecht University, P.O. Box 80082, 3508 TB Utrecht, the Netherlands.

2Polymer Laboratory, Chemistry Department, School of Science, University of Tehran, Tehran, Iran. 


\section{Abstract}

In this study, a new type of injectable hydrogel called "HyMic" that can convert into core crosslinked (CCL) micelles upon exposure to matrix metalloproteinases (MMP's), was designed and developed for drug delivery applications. HyMic is composed of CCL micelles connected via an enzyme cleavable linker. To this end, two complementary ABA block copolymers with polyethylene glycol (PEG) as B block were synthesized using atom transfer radical polymerization (ATRP). The A blocks were composed of a random copolymer of $\mathrm{N}$ isopropylacrylamide (NIPAM) and either N-(2-hydroxypropyl)methacrylamide- cysteine (HPMACys) or N-(2-hydroxypropyl) methacrylamide-ethylthioglycolate succinic acid (HPMA-ETSA). Mixing the aqueous solutions of the obtained polymers and rising the temperature above the cloud point of the PNIPAM block resulted in the self-assembly of these polymers into flower-like micelles composed of a hydrophilic PEG shell and hydrophobic core. The micellar core was crosslinked by native chemical ligation between the cysteine (in HPMA-Cys) and thioester (in HPMA-ETSA) functionalities. A slight excess of thioester to cysteine groups (molar ratio 3:2) was used to allow further chemical reactions exploiting the unreacted thioester groups. The obtained micelles displayed a Z-average diameter of $80 \pm 1 \mathrm{~nm}$ (PDI 0.1 ), and $\zeta$-potential of $-4.2 \pm 0.4 \mathrm{mV}$ and were linked using two types of pentablock copolymers of P(NIPAM-co-HPMA-Cys)-PEG-Peptide-PEG-P(NIPAM-co -HPMA-Cys) (PepNC) to yield hydrogels. The pentablock copolymers were synthesized using a PEG-peptidePEG ATRP macroinitiator and the peptide midblock (lysine-glycine -proline-glutamineisoleucine-phenylalanine-glycine-glutamine-lysine (Lys-Gly-Pro-Gln-Gly -Ile-Phe-Gly-GlnLys)) consisted of either L or D amino acids (L-Pep-NC or D-Pep-NC), of which the L-amino acid sequence is a substrate for matrix metalloproteases 2 and 9 (MMPs 2 and 9). Upon mixing of the CCL micelles and the linker (L/D-Pep-NC), the cysteine functionalities of the L/D-Pep-NC reacted with remaining thioester moieties in the micellar core via native chemical ligation yielding a hydrogel within 160 minutes as demonstrated by rheological measurements. As anticipated, the gel crosslinked with L-Pep-NC was degraded in 7-45 days upon exposure to metalloproteases in a concentration dependent manner, while the gel crosslinked with the D-Pep-NC remained intact even after 2 months. Dynamic light scattering analysis of the release medium revealed the presence of nanoparticles with a Zaverage diameter of $\sim 120 \mathrm{~nm}(\mathrm{PDI}<0.3)$ and $\zeta$-potential of $\sim-3 \mathrm{mV}$ indicating release of core crosslinked micelles upon HyMic exposure to metalloproteases. An in-vitro study demonstrated that the released CCL micelles were taken up by HeLa cells. Therefore, HyMic as an injectable and enzyme degradable hydrogel displaying controlled and on-demand 
release of $\mathrm{CCL}$ micelles has potential for intracellular drug delivery in tissues with upregulation of MMPs, e.g. in cancer tissues. 


\section{Introduction}

Hydrogels are three-dimensional networks of crosslinked hydrophilic polymers that can retain large amounts of waters while maintaining their structure ${ }^{1-2}$. Hydrogels have been extensively studied for delivery of a variety of therapeutics ranging from small molecules ${ }^{3}$ to large protein $s^{4-5}$ and nucleic acids ${ }^{6-7}$. Various water-soluble therapeutics can be loaded into the hydrogel matrixes during their formation ${ }^{8-9}$ or encapsulated in carriers such as polymeric nanoparticles ${ }^{10}$, liposomes ${ }^{11-12}$, or micelles ${ }^{13}$. Characteristics of the hydrogel such as pore size, swelling kinetics, and degradation mechanism play an important role in the release kinetics of loaded therapeutics ${ }^{14}$. Regarding the release of drug-loaded nanoparticles from hydrogels, characteristics of the nanoparticles, in particular size, charge, stability, as well as uniform distribution into the gel matrix, affect particle release kinetics ${ }^{15-}$ ${ }^{16}$. In recent years hydrogels that are converted into nanoparticles have gained interest for drug delivery applications. For instance, de Graaf et al. reported on the development of a drug-loaded hydrogel based on an ABA block polymer having $A$ blocks of poly $(N$ isopropylacrylamide) PNIPAM and a B block of polyethylene glycol (PEG). They demonstrated that this hydrogel gradually and spontaneously converts into drug-loaded flower-like micelles ${ }^{17}$. In other studies, micelles have been connected to each other using a linker exploiting e.g. an aldehyde and hydroxylamine reaction ${ }^{18}$, radical polymerization ${ }^{19}$, and metal-ligand interactions $\mathrm{s}^{20-21}$ to yield a macroscopic hydrogel structure. Although the mentioned systems showed interesting properties for drug delivery applications, they lack a triggered drug release mechanism upon disease induced stimuli.

Hydrogels can be designed as stimuli-responsive materials that respond to signals from the surrounding environment resulting in e.g. triggered drug release $\mathrm{e}^{22-24}$. Incorporation of functional groups or enzyme responsive blocks such as trans azobenzene ${ }^{25}, \mathrm{~N}$ isopropylacrylamide (NIPAM) ${ }^{26}$, acrylic acid ${ }^{27}$ or cleavable peptides ${ }^{28}$ in polymer structures can result in stimuli-responsive hydrogels. These materials can release the loaded therapeutics upon a trigger by e.g. light exposure, temperature or $\mathrm{pH}$ changes, or the presence of enzymes upregulated in diseased tissues and organs.

Among stimuli-responsive materials, enzyme degradable hydrogels have shown to exhibit autoregulated degradation and accordingly they release their drug payload (for drug delivery purposes) or enhance cell migration (for tissue engineering purposes) ${ }^{29-30}$. For instance, Burdick et $a^{\beta 1}$. reported a matrix metalloproteinase (MMPs) responsive hydrogel loaded with a recombinant tissue inhibitor of MMPs (rTIMP-3). This polysaccharide-based hydrogel was crosslinked by MMP cleavable peptides. The authors demonstrated that the presence of MMP caused degradation of the gel and consequently release of the loaded rTIMP-3, which in turn resulted in a reduction in MMP activity in the overexpressed region ${ }^{31}$. 
In another study, it was shown that MMP responsive blocks in a heparin/PEG hydrogel network in the presence of the matching enzyme enhanced cell viability and proliferation of mesenchymal stromal cells (MSC), which is important for cell-based cartilage regeneration ${ }^{32}$.

MMPs are upregulated in blood and tissues of patients with many types of human cancers $^{33,34}$. The expression of MMPs varies in different cancers as well as in different stages of the disease and can serve as a cancer biomarker ${ }^{35}$. MMPs promote cancer progression by supporting cancer cell proliferation, migration, invasion, metastasis, and angiogenesis ${ }^{36}$. Therefore, designing a therapeutic tool such as a hydrogel that undergoes degradation to yield nanoparticles triggered by overexpressed MMPs in pathological tissues, is of high interest for the intracellular delivery of e.g. anti-cancer drugs and biotherapeutics. In this contribution, an enzyme responsive hydrogel consisting of core-crosslinked (CCL) flowerlike micelles named "HyMic" is developed and investigated. To construct HyMic, core crosslinked flower-like micelles based on two complementary thermosensitive ABA triblock copolymers of P(NIPAM-co-HPMA-Cys)-PEG-P(NIPAM -co-HPMA-Cys) (PNC) and P(NIPAMCO-HPMA-ETSA)-PEG-P(NIPAM-CO-HPMA-ETSA) (PNE), were prepared using Native Chemical Ligation (NCL) as core-crosslinking method ${ }^{37}$. The formed micelles were subsequently linked together using a pentablock copolymer of P(NIPAM-co-HPMA-Cys)PEG-peptide-PEG-P(NIPAM-co-HPMA-Cys) (Pep-NC) yielding a micellar hydrogel network. The selected peptide block (Lys-Gly-Pro-Gln-Gly-Ile-Phe -Gly-Gln-Lys) is an MMP responsive sequence $^{38}$. Additionally, uptake of the released $\mathrm{CCL}$ micelles by HeLa cells (human epithelial cervix carcinoma cell line) was explored to investigate the possibility of intracellular drug delivery by HyMic.

\section{Materials and Methods}

\subsection{Materials}

All commercial chemicals were obtained from Sigma-Aldrich (Zwijndrecht, the Netherlands) and used as received unless indicated otherwise. $N$-(2-hydroxypropyl) methacrylamide (HPMA) was synthesized by a reaction of methacryloyl chloride with 1-aminopropan-2-ol in dichloromethane according to a previously published procedure ${ }^{39}$. Peptide grade dichloromethane (DCM), tetrahydrofuran (THF) and hexafluoro-2-propanol (HFIP) were obtained from Biosolve (Valkenswaard, the Netherlands). N-(2-hydroxypropyl) methacrylamide-Boc-S-acetamidomethyl-L-cysteine (HPMA-Boc-Cys(Acm)) and $\mathrm{N}$-(2hydroxypropyl)methacrylamide-ethylthioglycolate succinic acid (HPMA-ETSA) were synthesized as described previously ${ }^{37,40}$. Phosphate buffered saline 10X (PBS) pH 7.4 (1.37 $\mathrm{M} \mathrm{NaCl}, 0.027 \mathrm{M} \mathrm{KCl}$ and $0.119 \mathrm{M}$ phosphates) BioReagents were purchased from $\mathrm{B}$. Braun 
(Melsungen, Germany). Alexa Fluor ${ }^{\mathrm{TM}} 750$ and 568 C5 maleimide dyes were obtained from Thermo Fisher Scientific (Massachusetts, United States). Alpha-t-butyloxycarbonylamino $\omega$-carboxy succinimidyl ester poly(ethylene glycol) (Boc-NH-PEG-NHS) (PEG-Mn 3 kDa) was purchased from Iris Biotech GMBH (Marktredwitz, Germany). Acetylated $N$-terminal L and $D$ peptides (sequence: Lys-Gly-Pro-GIn-Gly-Ile-Phe-GLy-GIn-Lys) were purchased from GenScript (Leiden, the Netherlands). PD-10 desalting columns were purchased from GE Healthcare (Uppsala, Sweden). Dialysis tubes (molecular weight cutoff (MWCO) 3.5 and 10 kDa) were obtained from Fisher Scientific (Bleiswijk, the Netherlands). PEG standards (molecular weights ranging from 106 to $969000 \mathrm{Da}$ ) for GPC characterization were purchased from Agilent Technologies BV (Santa Clara, US).

\subsection{Polymer synthesis and characterization}

\subsubsection{Synthesis of D or L-Pep-(NH2-PEG $3 \mathrm{k})_{2}$}

D and L-peptides (40 mg, $36 \mu \mathrm{mol}$ ) (lysine-glycine-proline-glutamine-isoleucinephenylalanine-glycine-glutamine-lysine) (Lys-Gly-Pro-Gln-Gly-Ile-Phe-Gly-Gln-Lys), figure 1) were separately dissolved in $2 \mathrm{~mL}$ of DMSO followed by the addition of $60 \mu \mathrm{L}$ triethylamine. Subsequently, Boc-NH-PEG-NHS (221 mg, $72 \mu \mathrm{mol})$ was added and dissolved in the reaction mixture and allowed to react for 48 hours at room temperature. The crude product, D or L-Pep-(Boc-NH-PEG $\left.{ }_{3 k}\right)_{2}$ was dialyzed against water (MWCO, $3.5 \mathrm{kDa}$ ) at room temperature for two days and subsequently lyophilized. The obtained product was analyzed by ${ }^{1} \mathrm{H}-\mathrm{NMR}$ and GPC (NMR: Fig. 2, SI-Fig.2B and GPC: Fig. 3).

To remove the Boc protecting group from L/D-Pep-(Boc-NH-PEG)2, $200 \mathrm{mg}$ of the polymers were dissolved in a $4 \mathrm{~mL}$ solution of dry DCM and trifluoroacetic acid (TFA) (1:3 v/v) and left to react for 1 hour at room temperature. Subsequently, the solvents were evaporated, and the residues were dissolved in water and dialyzed against water (MWCO, $3.5 \mathrm{kDa}$ ) for two days at room temperature and then lyophilized. The obtained products were characterized by GPC (Fig. 3) and ${ }^{1} \mathrm{H}-\mathrm{NMR}$ (Fig. 3, SI-Fig. 2).

\subsubsection{Synthesis of D- and L-Pep-(Br-C(CH3) $\left.)_{2}-\mathrm{CO}-\mathrm{NH}-\mathrm{PEG} \mathrm{G}_{3}\right)_{2}$ macroinitiator for ATRP}

D and L-Pep- $\left(\mathrm{NH}_{2}-\mathrm{PEG}_{3 \mathrm{k}}\right)_{2}(220 \mathrm{mg}, 30 \mu \mathrm{mol})$ (section 2.2.1) were separately dissolved in 9 $\mathrm{mL}$ dry THF followed by the addition of $1 \mathrm{~mL}$ hexafluoro-2-propanol (HFIP). Under a nitrogen atmosphere, triethylamine $(20 \mu \mathrm{L})$ and $\alpha$-bromoisobutyryl bromide $(15 \mu \mathrm{L}, 120 \mu \mathrm{mol})$ ( $2 \mathrm{eq}$. to the amine end groups of D- and L-Pep- $\left.\left(\mathrm{NH}_{2}-\mathrm{PEG}_{3 \mathrm{k}}\right)_{2}\right)$ were added and the reaction mixtures were stirred overnight at room temperature. Next, the formed ammonium 
bromide salts were filtered off and the solvents were evaporated under reduced pressure. The crude products were dissolved in water and the obtained solutions were dialyzed against water (MWCO, $3.5 \mathrm{kDa}$ ) for 2 days and subsequently lyophilized. The obtained macroinitiators were characterized by ${ }^{1} \mathrm{H}-\mathrm{NMR}$ (Fig. 2, SI-Fig. 2C, 3) and GPC (Fig. 3, SI-Fig.4).

\subsubsection{Synthesis of Peptide-(PEG-P(NIPAM-co-HPMA-Cys))2, Pep-NC}

$D$ and L-Pep-(Br-C(CH$\left.)_{2}-\mathrm{CO}-\mathrm{NH}-\mathrm{PEG}_{3 \mathrm{k}}\right)_{2}$ macroinitiators (section 2.2.2) (60 mg, $8.0 \mu \mathrm{mol}$ ), together with $\mathrm{CuBr}(4.5 \mathrm{mg}, 31 \mu \mathrm{mol}), \mathrm{CuBr}_{2}(4.7 \mathrm{mg}, 21 \mu \mathrm{mol}), \operatorname{NIPAM}(264 \mathrm{mg} ; 2.3 \mathrm{mmol})$ and HPMA-Cys ( $67 \mathrm{mg}, 0.16 \mathrm{mmol}$ ) were separately dissolved in a mixture of $2.0 \mathrm{~mL}$ water and $0.84 \mathrm{~mL}$ acetonitrile ${ }^{41}$. The mixtures were stirred and deoxygenated by flushing with nitrogen for 15 minutes at room temperature, followed by 15 minutes in an ice bath. Polymerization started after the addition of $16 \mu \mathrm{L}(0.06 \mathrm{mmol})$ of tris[2(dimethylamino)ethyl]amine (Me 6 TREN) and the reaction mixtures were stirred for 2 hours in an ice bath. Next, the formed polymers were diluted in water to $15 \mathrm{~mL}$, then dialyzed (MWCO, $10 \mathrm{kDa}$ ) against water at room temperature for one day and subsequently lyophilized and analyzed by ${ }^{1} \mathrm{H}-\mathrm{NMR}$ (SI-Fig. 5A) and GPC (Fig. 3). Finally, the Acm and Boc protecting groups of cysteine were removed as described previously ${ }^{42}$. Briefly, Boc protecting group of cysteines were removed by dissolving $250 \mathrm{mg}$ of the polymer in dry DCM and trifluoroacetic acid (TFA) $(1: 1 \mathrm{v} / \mathrm{v}, 10 \mathrm{~mL})$ and the mixture was stirred for 1 hour at room temperature in a nitrogen atmosphere. Subsequently, the solvent was evaporated under reduced pressure and the crude product was dissolved in $2 \mathrm{~mL} D C M$ and subsequently precipitated in cold diethyl ether. The Acm protecting groups were removed by dissolving $250 \mathrm{mg}$ of the Boc deprotected polymer in methanol (MeOH) and water (1:1 $\mathrm{v} / \mathrm{v}, 10 \mathrm{~mL}$ ) under a nitrogen atmosphere followed by the addition of $500 \mu \mathrm{L} \mathrm{HCl}(1 \mathrm{M})$ and $1 \mathrm{~mL}$ iodine $0.2 \mathrm{M}$ in $\mathrm{MeOH}$. The mixture was stirred for $1 \mathrm{~h}$ at room temperature, followed by the addition of $1 \mathrm{~mL}$ of ascorbic acid $(1 \mathrm{M})$ in water to quench the excess of iodine. Subsequently, excess of tris(2-carboxyethyl)phosphine (TCEP) (250 mg) was added to reduce the formed disulfide bonds. The mixture was stirred overnight, dialyzed against water for 2 days at room temperature (MWCO, $10 \mathrm{kDa}$ ), and the final product was obtained after lyophilization. The obtained product was analyzed by ${ }^{1} \mathrm{H}-\mathrm{NMR}$ (SI-Fig 5B) and GPC (SIFig. 9). The same method was used for deprotection of (NIPAM-co-HPMA-Cys)-PEG-DPeptide-PEG-P(NIPAM-co-HPMA-Cys) (D-Pep-NC). The obtained polymer was characterized by ${ }^{1} \mathrm{H}-\mathrm{NMR}$ (SI-Fig. $6 \mathrm{~A}$ and $6 \mathrm{~B}$ ) and GPC (Fig. 3, SI-Fig. 9).

\subsubsection{Synthesis of P(NIPAM-co-HPMA-Cys)-PEG-P(NIPAM-co-HPMA-Cys)(PNC) and P(NIPAM-co-HPMA-ETSA)-PEG-P(NIPAM-co-HPMA-ETSA)(PNE)}


The polymerization solvent for the synthesis of PNC was a mixture of $2.8 \mathrm{~mL}$ of water and $0.9 \mathrm{~mL}$ acetonitrile. For the synthesis of PNE, a mixture of $2.5 \mathrm{~mL}$ water, $0.6 \mathrm{~mL}$ acetonitrile, and $1.3 \mathrm{~mL}$ of DMSO was used. Poly(ethylene glycol) bis(2-bromoisobutyrate) ${ }^{37}$ (50 mg, 7.9 $\mu \mathrm{mol}), \mathrm{CuBr}(4.5 \mathrm{mg}, 31 \mu \mathrm{mol}), \mathrm{CuBr}_{2}(4.7 \mathrm{mg}, 21 \mu \mathrm{mol}), \operatorname{NIPAM}(264 \mathrm{mg} ; 2.3 \mathrm{mmol}$ ) and either HPMA-ETSA (56 mg, $0.16 \mathrm{mmol}$ ) for synthesis of PNE or HPMA-Boc-Cys(Acm) for synthesis of PNC (67 mg, $0.16 \mathrm{mmol}$ ) were dissolved in the corresponding polymerization solvent. Next, the mixtures were deoxygenated by flushing with nitrogen for 15 minutes at room temperature and subsequently for 15 minutes upon cooling in an ice bath. To initiate the polymerization, the ligand Me $\mathrm{C}_{\mathrm{TREN}}(16 \mu \mathrm{L}, 60 \mu \mathrm{mol})$ was added to the solution, and the reaction mixture was stirred for 2 and $5 \mathrm{~h}$ in an ice bath for PNC and PNE, respectively. The final products were diluted with $15 \mathrm{~mL}$ water and subsequently dialyzed (MWCO, 10 $\mathrm{kDa}$ ) against water at room temperature for one day and lyophilized. The obtained polymers were characterized by ${ }^{1} \mathrm{H}-\mathrm{NMR}$ (PNE: SI-Fig. 7, protected PNC: SI-Fig. 8A) and GPC (PNE: SIFig. 10, PNC: SI-Fig. 11A).

Finally, the Acm and Boc protecting groups of cysteine in protected PNC were removed as described in section 2.2.3. The obtained PNC was characterized by ${ }^{1} \mathrm{H}-\mathrm{NMR}$ (SI-Fig. 8B) and GPC (SI-Fig. 11B).

\subsubsection{NMR spectroscopic analysis}

The obtained polymers were characterized by ${ }^{1} \mathrm{H}$-NMR using a Bruker 600 UltraShield ${ }^{\mathrm{TM}}$ spectrometer (Billerica, Massachusetts, USA). The chemical shifts were calibrated against residual solvent peaks of $\mathrm{CHCl}_{3}(\delta=7.26 \mathrm{ppm})$ and $\mathrm{DMSO}(\delta=2.50 \mathrm{ppm})$.

\subsubsection{Gel permeation chromatography (GPC) analysis}

The molecular weights and molecular weight distribution of the synthesized polymers were determined by GPC using a Waters Alliance 2414 System (Waters Corporation, Milford, MA) equipped with a refractive index detector. The separation was performed using 2 PLgel 5 $\mu \mathrm{m}$ Mixed-D columns (Polymer Laboratories, UK) at a temperature of $65{ }^{\circ} \mathrm{C}$ and DMF containing $10 \mathrm{mM} \mathrm{LiCl}$ at a flow of $1 \mathrm{~mL} \cdot \mathrm{min}^{-1}$ was used as mobile phase ${ }^{43}$. Samples were prepared in the same solvent at a concentration of $5 \mathrm{mg} \cdot \mathrm{mL}^{-1}$ and $50 \mu \mathrm{L}$ was injected into the column for each measurement. A series of linear PEGs with narrow and defined molecular weights were used as calibration standards. Data were recorded and analyzed with Empower software v. 3. 2010.

\subsubsection{Determination of cloud points of polymers}


The cloud point (CP), defined as the onset of increasing scattering intensity, was measured using a Jasko FP-8300 spectrofluorometer (JASCO, Tokyo, Japan) at $650 \mathrm{~nm}$. The polymers

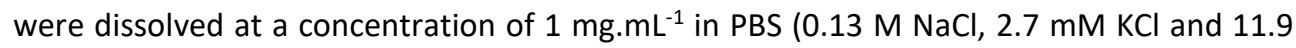
$\mathrm{mM}$ phosphates $\mathrm{pH}$ 7.4). The scattering intensity was monitored while heating the sample from 10 to $50{ }^{\circ} \mathrm{C}$ at $1{ }^{\circ} \mathrm{C}$ per minute.

\subsubsection{Kinetics of Pep-NC cleavage}

To investigate the cleavage rate of the peptide in the Pep-NC polymer, $40 \mathrm{mg}$ of L-Pep-NC polymer was dissolved in $8 \mathrm{ml}$ of PBS $(0.13 \mathrm{M} \mathrm{NaCl}, 2.7 \mathrm{mM} \mathrm{KCl}, 11.9 \mathrm{mM}$ phosphates, 0.9 $\mathrm{mM} \mathrm{CaCl}_{2}, 0.02 \% \mathrm{NaN}_{3}, \mathrm{pH} \mathrm{7.4)}$ at a concentration of $5 \mathrm{mg}^{-\mathrm{mL}^{-1}}$ at $4{ }^{\circ} \mathrm{C}$ for $3 \mathrm{~h}$ and subsequently at $37{ }^{\circ} \mathrm{C}$. Metalloprotease (type IV) collagenase from C. histolyticume (a mixture of enzymes with molecular weight distribution 63-130 kDa) was used as a model enzyme for MMP-2 and MMP-944. Subsequently, polymer solutions were incubated with collagenase at a concentration of 0.5 units. $\mathrm{mL}^{-1}$ at $37^{\circ} \mathrm{C}$ (this concentration is close to the total tissue concentration of MMP-1 and MMP-9 $\left(500 \mathrm{ng} \cdot \mathrm{mL}^{-1}\right)$ reported for breast cancer $)^{45}$. At several time points, $1 \mathrm{~mL}$ samples were withdrawn and diluted with $1 \mathrm{~mL}$ of an ethylenediaminetetraacetic acid (EDTA) solution (0.1 M) in PBS $(0.13 \mathrm{M} \mathrm{NaCl}, 2.7 \mathrm{mM} \mathrm{KCl}$, $11.9 \mathrm{mM}$ phosphates, $\mathrm{pH} 7.4$ ) and incubated for $1 \mathrm{~h}$ at room temperature. EDTA inhibits the enzyme activity via chelation of the metal ions (calcium and magnesium) required for catalytic activity ${ }^{46,47}$. Next, the samples were dialyzed against water (MWCO, 10 kDa) for 2 days at $4{ }^{\circ} \mathrm{C}$ and subsequently lyophilized. D-Pep-NC at a polymer concentration of $5 \mathrm{mg} \cdot \mathrm{mL}^{-}$

${ }^{1}$ was dissolved in $1 \mathrm{~mL}$ PBS $\left(0.13 \mathrm{M} \mathrm{NaCl}, 2.7 \mathrm{mM} \mathrm{KCl}, 11.9 \mathrm{mM}\right.$ phosphates, $0.9 \mathrm{mM} \mathrm{CaCl}_{2}$, $0.02 \% \mathrm{NaN}_{3}, \mathrm{pH} 7.4$ ) and incubated with collagenase at a concentration of 30 units. $\mathrm{mL}^{-1}$ for $24 \mathrm{~h}$. Next, the sample was treated with $1 \mathrm{~mL}$ of EDTA solution $(0.1 \mathrm{M})$ for $1 \mathrm{~h}$ and then dialyzed against water (MWCO, $10 \mathrm{kDa}$ ) for 2 days at $4{ }^{\circ} \mathrm{C}$ and subsequently lyophilized. The obtained polymers were dissolved at a concentration of $5 \mathrm{mg} \cdot \mathrm{mL}^{-1}$ in DMF containing 10 $\mathrm{mM} \mathrm{LiCl}$ and the molecular weights were analyzed by GPC (section 2.2.6). The results are shown in Fig. 4 and SI-Fig. 12.

\subsection{Micelle preparation and characterization}

\subsubsection{Micelle preparation}

Micelles were formed by a fast heating method as follows: PNC and PNE were dissolved separately in PBS (0.13 M NaCl, $2.7 \mathrm{mM} \mathrm{KCl}$ and $11.9 \mathrm{mM}$ phosphates, $\mathrm{pH} 7.4$ ) at a concentration of $10 \mathrm{mg} \cdot \mathrm{mL}^{-1}$ and left at $4{ }^{\circ} \mathrm{C}$ for 3 hours. Subsequently, the solutions were mixed in a 2:3 (PNC:PNE) volume ratio (i.e. the molar ratio of HPMA-Cys to HPMA-ETSA was 
2:3). Subsequently, the mixture was heated to $50{ }^{\circ} \mathrm{C}$ using an oil bath and stirred at this temperature overnight. The resulting micellar dispersion was dialyzed against water (MWCO, $10 \mathrm{kDa}$ ) for two days at room temperature and subsequently lyophilized. The obtained micelles were characterized by DLS before and after lyophilization (Fig. 7-A, 7-C).

\subsubsection{Preparation of fluorescently labeled micelles}

Micelles were formed following the same procedure as described in section 2.3.1. Then, the buffer medium was refreshed using a PD-10 desalting column equilibrated with PBS $(0.13$ $\mathrm{M} \mathrm{NaCl}, 2.7 \mathrm{mM} \mathrm{KCl}$ and $11.9 \mathrm{mM}$ phosphates, $\mathrm{pH}$ 7.4) according to the method provided by the supplier. Subsequently, $10 \mu \mathrm{L}$ of a $20 \mu \mathrm{g} \cdot \mathrm{mL}^{-1}$ maleimide-Alexa fluor C5 750 or C5 568 solution in DMSO was added to $1 \mathrm{~mL}$ of micellar dispersion $\left(10 \mathrm{mg} \cdot \mathrm{mL}^{-1}\right)$ and left to react at $4{ }^{\circ} \mathrm{C}$ for two days. Next, the labeled micelles were dialyzed against water (MWCO, $10 \mathrm{kDa}$ ) at room temperature for one day and subsequently purified three times by PD-10 columns equilibrated with deionized water and finally lyophilized.

\subsubsection{Dynamic light scattering (DLS)}

The size of the obtained micelles at a concentration of $1 \mathrm{mg} \cdot \mathrm{mL}^{-1}$ in PBS $(0.13 \mathrm{M} \mathrm{NaCl}, 2.7$ $\mathrm{mM} \mathrm{KCl}$ and $11.9 \mathrm{mM}$ phosphates, $\mathrm{pH}$ 7.4) was measured by DLS using a Zetasizer Nano $\mathrm{S}$ (ZEN 1600) (Malvern Instruments Ltd., Malvern, UK) equipped with a He-Ne 4 mW, 632.8 $\mathrm{nm}$ laser. The measurements were carried out at a $173^{\circ}$ angle at $37^{\circ} \mathrm{C}$ controlled by the instrument. The Z-average and polydispersity index was calculated by Zetasizer software v7.13. The solvent viscosity was corrected for temperature changes by the software.

\subsubsection{Zeta potential}

The obtained micelles in PBS were diluted (1:20) in HEPES (20 mM, pH 7.0) to the final concentration of $0.5 \mathrm{mg} \cdot \mathrm{mL}^{-1}$ and their Zeta potential was measured at $37{ }^{\circ} \mathrm{C}$ using a Zetasizer Nano Z, (Malvern Instruments Ltd., Malvern, UK).

\subsection{HyMic preparation, characterization, and degradation}

\subsubsection{Preparation of micellar hydrogel (HyMic)}

HyMic was prepared at a total polymer concentration of $20 \mathrm{wt} \%$ as follows: $6 \mathrm{mg}$ of lyophilized micelles were weighed in a $1.5 \mathrm{~mL}$ Eppendorf vial followed by the addition of 15 $\mu \mathrm{L}$ of PBS (0.13 M NaCl, $2.7 \mathrm{mM} \mathrm{KCl}$ and $11.9 \mathrm{mM}$ phosphates, $\mathrm{pH} 7.4)$, and hydrated for 1 $\mathrm{h}$ at $4{ }^{\circ} \mathrm{C}$. In a separate vial, $15 \mu \mathrm{L}$ of PBS was added to $1.3 \mathrm{mg}$ of D- or L-Pep-NC (23 wt\% of the amount of micelles) and left to dissolve for $1 \mathrm{~h}$ at $4{ }^{\circ} \mathrm{C}$. Next, the D- or L-Pep-NC solution 
was added to the micellar dispersions and the mixtures were incubated at $4{ }^{\circ} \mathrm{C}$ for $1 \mathrm{~h}$ and subsequently incubated for $6 \mathrm{~h}$ at $37^{\circ} \mathrm{C}$ for hydrogel formation.

\subsubsection{Rheological characterization}

Rheological analysis of the hydrogel samples and micellar dispersions was performed on a DHR-2 rheometer (TA Instrument, New Castel, DE) using a $20 \mathrm{~mm}$ aluminum cone $\left(1^{\circ}\right)$ geometry equipped with a solvent trap. Time sweeps were performed for 3 hours at $37^{\circ} \mathrm{C}$ at a frequency of $1 \mathrm{~Hz}$ and $1 \%$ strain. For each measurement, $70 \mu \mathrm{L}$ samples were used.

\subsubsection{Swelling and degradation study}

HyMic (fluorescently labeled) hydrogels with a volume of $\sim 30 \mu \mathrm{L}$ were prepared as described in section 2.4.1. The obtained hydrogels were transferred into $2 \mathrm{~mL}$ glass vials and the gel weights were recorded $\left(W_{0}\right)$. The samples were then immersed into $500 \mu \mathrm{L}$ of PBS (0.13 M NaCl, $2.7 \mathrm{mM} \mathrm{KCl}, 11.9 \mathrm{mM}$ phosphates, $0.9 \mathrm{mM} \mathrm{CaCl}_{2}, 0.02 \% \mathrm{NaN}_{3}, \mathrm{pH} 7.4$ ) with $0.0,7.5,15.0$ or 30.0 units. $\mathrm{mL}^{-1}$ of collagenase and incubated at $37^{\circ} \mathrm{C}$. At regular time points, the medium was removed, and the weight of the gel was recorded $\left(W_{t}\right)$, and subsequently, $500 \mu \mathrm{L}$ of fresh medium was added and the samples were further incubated at $37^{\circ} \mathrm{C}$. The gel release medium was analyzed by DLS for size, polydispersity index (PDI), and derived count rate of the released core crosslinked micelles. In addition, fluorescence intensity of the supernatant was analyzed as described in section 2.4.4. The swelling and degradation was recorded three times a week until complete degradation or until 2 months after which the experiment was stopped. The swelling ratio $\left(S R=W_{t} / W_{0}\right)$ is reported as the weight of the gel at a certain time point $\left(W_{t}\right)$ divided by the initial gel weight $\left(W_{0}\right)$. The mesh sizes of the hydrogels were estimated based on the following equation:

$$
\xi=\left(\frac{\mathrm{G}^{\prime} N_{A}}{R T}\right)^{-\frac{1}{3}}
$$

where $N_{A}$ is Avogadro constant, $R$ is the molar gas constant, and $T$ is temperature.

For cell internalization study, hydrogels composed of Cy5-conjugated micelles were made using the L-Pep-NC linker. These samples were incubated with collagenase at a concentration of 10 units. $\mathrm{mL}^{-1}$. The fluorescence intensity of the medium was measured in time to quantify the concentration of the released micelles. On day 21 , the concentration of the released $\mathrm{CCL}$ micelles reached $\sim 8 \mathrm{mg} \cdot \mathrm{mL}^{-1}$. The released micelles were used for incubation with cells as detailed in section 2.5 . 


\subsubsection{Fluorescence intensity measurement}

To measure the fluorescent signals of Cy7 labeled micelles in the gel release medium, $30 \mu \mathrm{L}$ of the release medium was transferred into a clear 384-well plate and analyzed using Odyssey infrared scanner (LI-COR Biosciences, Westburg, the Netherlands) at $700 \mathrm{~nm}$. To measure the fluorescent intensity of the Cy5-conjugated micelles (released from hydrogel and used in cell study), $100 \mu \mathrm{L}$ of release medium was transferred into a black 384-well plate and analyzed using a Jasko FP-8300 spectrofluorometer (JASCO, Tokyo, Japan). The excitation and emission wavelengths were set at 578 and $603 \mathrm{~nm}$, respectively. Standard curves of the corresponding dye conjugated micelles were used for quantification of fluorescent signals.

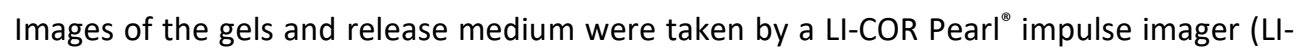
COR, Lincoln, Nebraska, USA).

\subsection{Cellular internalization study}

Cellular uptake of the released micelles was investigated using HeLa cells. The cells were seeded in a glass-bottomed 96 well-plate at a density of 8000 cells/well and incubated at $37{ }^{\circ} \mathrm{C}$ for $24 \mathrm{~h}$. Then, the fluorescently labeled micelles either released from the gel or control micelles (freshly prepared micelles that were not converted into a gel) were added at a concentration of $400 \mu \mathrm{g} \cdot \mathrm{mL}^{-1}$ for 1,6 and $24 \mathrm{~h}$ at $37^{\circ} \mathrm{C}$. The cells were washed twice with PBS (0.13 M NaCl, $2.7 \mathrm{mM} \mathrm{KCl}, 11.9 \mathrm{mM}$ phosphates, $\mathrm{pH} 7.4$ ) and the plate was transferred into a Yokogawa CV7000 (Tokyo, Japan) spinning disk microscope with a 60x 1.2NA water objective.

\section{Results and Discussion}

\subsection{Polymer synthesis and characterization}

\subsubsection{Synthesis and characterization of Peptide-PEG ATRP macroinitiator}

Figure $1 \mathrm{~A}$ shows the three-step synthesis route of the PEG-Pep-PEG atom transfer radical polymerization (ATRP) ${ }^{48}$ macroinitiator. The commercial Boc-NH-PEG-NHS (Fig. 1, step A-1, compound 2) was characterized by GPC and ${ }^{1} \mathrm{H}-\mathrm{NMR}$. NMR analysis confirmed the presence of tert-butyloxycarbonyl (Boc) and succinimidyl ester (NHS) groups in compound 2 with a molar ratio of 1:1 (SI-Fig. 2A). GPC characterization displayed a peak at 15.6 minutes corresponding to polymer with a number-average molecular weight $\left(\mathrm{M}_{n}\right)$ of $3.4 \mathrm{kDa}$ in agreement with the specifications of the supplier and a shoulder at a retention time of 14.7 minutes ( $7 \%$ of the total peak area), corresponding with a polymer of $M_{n}=7.3 \mathrm{kDa}$ (Fig. 3). 
This shoulder can most likely be attributed to the presence of PEG chains of higher molecular weight possibly also derivatized with NHS and Boc functionalities. The two free amines of the $\mathrm{N}$-terminus acetylated Lys-Gly-Pro-Gln-Gly-Ile-Phe-Gly-Gln-Lys were conjugated to the NHS-end group of PEG (Fig. 1, step A-1). After dialysis and lyophilization, PEG-Pep-PEG (Fig. 1, step A-1, compound 3) was obtained in a yield of $87 \%$. The ${ }^{1} \mathrm{H}-\mathrm{NMR}$ spectrum showed that the molar ratio of the phenyl group of phenylalanine in the peptide sequence and Boc group in the PEG was 1:2 (Fig. 2-2) (SI-Fig. 2B) demonstrating the successful synthesis of the macroinitiator. Additionally, GPC analysis showed that the retention time of compound 3 was shifted to a lower retention time representing a polymer of higher molecular weight ( $\left.\mathrm{Mn}_{\mathrm{n}}: 7.8 \mathrm{kDa}\right)$ (Fig. 3), which again confirms the formation of PEG-Pep-PEG. A small peak with a retention time of 15 minutes ( $7 \%$ of the total peak area) was detected, which corresponds to non-conjugated PEG ( $\mathrm{Mn}_{\mathrm{n}}$ 3.4 kDa), while the peak at 13.8 minutes may be assigned to conjugation of peptide to the higher $M_{n}$ PEG derivative present in the commercial starting compound 2. The Boc groups at both ends of the PEGPep-PEG were removed by trifluoroacetic acid (TFA) and the crude product was dialyzed and lyophilized to result in deprotected PEG-Pep-PEG in a yield of $79 \%$ (Fig. 1, step A-2, compound 4). ${ }^{1} \mathrm{H}-\mathrm{NMR}$ analysis of compound 4 showed that the signal corresponding to Boc at $\delta=1.4$ had indeed disappeared (Fig. 2-3) demonstrating the quantitative removal of Boc groups. As expected, the molecular weight of the deprotected PEG-Pep-PEG ( $\left.\mathrm{M}_{\mathrm{n}}: 8.1\right)$ was similar to the Boc protected PEG-Pep-PEG ( $\mathrm{Mn}_{\mathrm{n}}: 8.3 \mathrm{kDa}$ ) (Fig. 3). It should be noted that the acetyl protecting group of the $N$-terminal peptide is stable under the deprotection procedure applied ${ }^{49}$, which means that there is no risk for functionalization of this moiety by ATRP initiator. To functionalize the PEG-Pep-PEG with an ATRP initiator group at both chain ends, the free terminal amine groups were reacted with $\alpha$-bromoisobutyryl bromide to result in a PEG-Pep-PEG ATRP macroinitiator with a yield of $78 \%$ (Fig. 1, step A-3, compound 6). The presence of the ${ }^{1} \mathrm{H}-\mathrm{NMR}$ signal at $1.8 \mathrm{ppm}$ belonging to the methyl groups of the ATRP initiator (12 protons per polymer chain) confirmed that all chains were functionalized with an ATRP initiator (Fig. 2-4) (SI-Fig. 2C). GPC and ${ }^{1} \mathrm{H}-\mathrm{NMR}$ showed an $\mathrm{Mn}_{\mathrm{n}}$ of 8.6 , and $7.5 \mathrm{kDa}$ for this macroinitiator, respectively. As a control, the same peptide sequence made of unnatural amino acids (D-amino acids) was conjugated to PEG and subsequently to the ATRP initiator. The resulting initiator was obtained in the yield of $71 \%$ and had an $\mathrm{Mn}_{\mathrm{n}}$ of $7.9 \mathrm{kDa}$ as determined by GPC (SI-Fig. 4). ${ }^{1} \mathrm{H}-\mathrm{NMR}$ analysis confirmed quantitative modification of the chain ends by $\alpha$-bromoisobutyryl bromide (SI-Fig. 3 ).

\subsubsection{Synthesis and characterization of thermosensitive ABCBA pentablock copolymers and ABA triblock copolymers}


The pentablock ( $A B C B A$ ) copolymers were synthesized by ATRP using the above described PEG-Pep-PEG macroinitiator (PEG as B block and peptide as $C$ block) (section 3.1.1). The polymer structure is shown in figure $1 \mathrm{~B}$, SI-Fig. 1, and the polymer characteristics are summarized in table 1. The A blocks consisting of NIPAM (N) and HPMA-Cys (C) with a feed molar ratio of 93:7 were polymerized from either the $L$ or D-peptide-PEG macroinitiator ( $L$ Pep or D-Pep) using a previously established method ${ }^{37}$ (Fig. 1B). In the present work, the resulting copolymers are referred to as L-Pep-NC and D-Pep-NC. Incorporation of HPMACys in the thermosensitive domain provides cysteine functionalities that can be exploited for native chemical ligation $(\mathrm{NCL})^{50}$. After polymerization, the final products were obtained after dialysis and lyophilization in high yields (82-87\%).

Two complementary thermosensitive triblock copolymers of P(NIPAM-co-HPMA-Cys)-PEGP(NIPAM-Co-HPMA-CYS) (PNC) and P(NIPAM-Co-HPMA-ETSA)-PEG-P(NIPAM-Co-HPMAETSA) (PNE) (SI-Fig. 1) are needed for micelle formation, as reported before ${ }^{37}$. PNC and PNE were synthesized by ATRP using a PEG ATRP macroinitiator ${ }^{37}\left(\mathrm{Mn}_{\mathrm{n}}: 6 \mathrm{kDa}\right)$ and a feed molar ratio of NIPAM:HPMA-Cys or NIPAM:ETSA of 93:7. After polymerization and purification by dialysis, the final polymers (protected) PNC and PNE were obtained in yields of 93 and $88 \%$, respectively. The $\mathrm{Mn}_{\mathrm{n}}$ 's as determined by GPC were higher than $\mathrm{Mn}^{\prime}$ 's obtained by NMR analysis, which has been observed before for PNIPAM based (co)polymers due to inter- and intramolecular hydrogen bonding ${ }^{51,52}$. The PDIs of the obtained polymers (1.4-1.7) were similar to what has been reported for these types of polymers. The different reactivity of acrylamide (NIPAM) and methacrylamide (HPMA) has shown to result in blocky structures in PNC and PNE polymers rather than a random distribution of the two types of monomers ${ }^{37}$.

The cysteine moieties in HPMA-Cys carry two protecting groups, namely a Boc group on the terminal amine groups and an Acm group on the thiol side chain groups. Therefore, the polymers containing HPMA-Cys groups underwent deprotection of Boc groups under acidic conditions and an inert atmosphere ${ }^{53}$. Thiol protecting groups (Acm) were removed by first oxidation by iodine under acidic conditions and were subsequently reduced to free thiols using TCEP ${ }^{40,54}$.

The synthesized PNC and PNE polymers exhibit a cloud point (CP) in aqueous solutions, attributed to the thermosensitive PNIPAM block ${ }^{26}$, at 34.1 and $29.2^{\circ} \mathrm{C}$, respectively, which is similar to values reported before for the same polymers ${ }^{37}$. The CPs of the Pep-NC polymers were very similar (31.8 and $31.4^{\circ} \mathrm{C}$ for L and D-Pep-NC, respectively) and slightly lower than the $\mathrm{CP}$ of PNC $\left(34.1^{\circ} \mathrm{C}\right)$ likely because the peptide sequence in Pep-NCs contains several hydrophobic amino acid residues ${ }^{55}$ (Table 1 ). 
Table 1: Characteristics of PNC and PNE ABA triblock copolymers containing a PEG B-block of 6 kDa. ABCBA pentablock copolymers: L-Pep-NC and D-Pep-NC containing L-Pep-(PEG $\left.G_{3 k}\right)_{2}$ or D-Pep-(PEG $\left.G_{3 k}\right)_{2}$ as mid BCB-blocks, respectively. The outer blocks of the different polymers are composed of either NIPAM and HPMA-Boc-Cys-(Acm) (PNC, L-Pep-NC, and D-Pep-NC) or NIPAM and HPMA-ETSA (PNE). In all polymerizations, the feed molar ratio of NIPAM to either HPMA-Boc-Cys-(Acm) or HPMA-ETSA was 93:7.

\begin{tabular}{|c|c|c|c|c|c|c|c|}
\hline \multirow[b]{2}{*}{ Polymer } & \multicolumn{2}{|c|}{ Obtained molar ratio ${ }^{a}$} & \multirow{2}{*}{$\begin{array}{l}\mathrm{Mn}^{\mathrm{a}} \\
(\mathrm{kDa})\end{array}$} & \multirow{2}{*}{$\begin{array}{l}\mathrm{Mn}^{\mathrm{b}} \\
(\mathrm{kDa})\end{array}$} & \multirow[b]{2}{*}{$\mathrm{PDI}^{\mathrm{b}}$} & \multirow[b]{2}{*}{$\begin{array}{l}\mathrm{CP} \\
\left({ }^{\circ} \mathrm{C}\right)\end{array}$} & \multirow{2}{*}{$\begin{array}{l}\text { Yield } \\
(\%)\end{array}$} \\
\hline & $\begin{array}{c}\text { [NIPAM]: } \\
{[\text { HPMA-Boc-Cys-(Acm)] }}\end{array}$ & $\begin{array}{c}\text { [NIPAM]: } \\
\text { [HPMA-ETSA] }\end{array}$ & & & & & \\
\hline PNC & 91:9 & - & 43.6 & 58.9 & 1.42 & $34.1^{\mathrm{C}}$ & 93 \\
\hline PNE & - & $92: 8$ & 40.1 & 64.1 & 1.78 & 29.2 & 88 \\
\hline L-Pep-NC & $90: 10$ & - & 44.8 & 62.5 & 1.75 & $31.8^{\mathrm{C}}$ & 87 \\
\hline D-Pep-NC & $91: 9$ & - & 44.2 & 64.3 & 1.72 & $31.4^{c}$ & 82 \\
\hline
\end{tabular}

a Determined by ${ }^{1}$ H-NMR. ${ }^{b}$ Determined by GPC. ${ }^{c}$ Cloud point of the deprotected polymer. 
A-1. Synthesis of D- or L-Pep-(Boc-NH-PEG $\left.{ }_{3 \mathrm{k}}\right)_{2}$
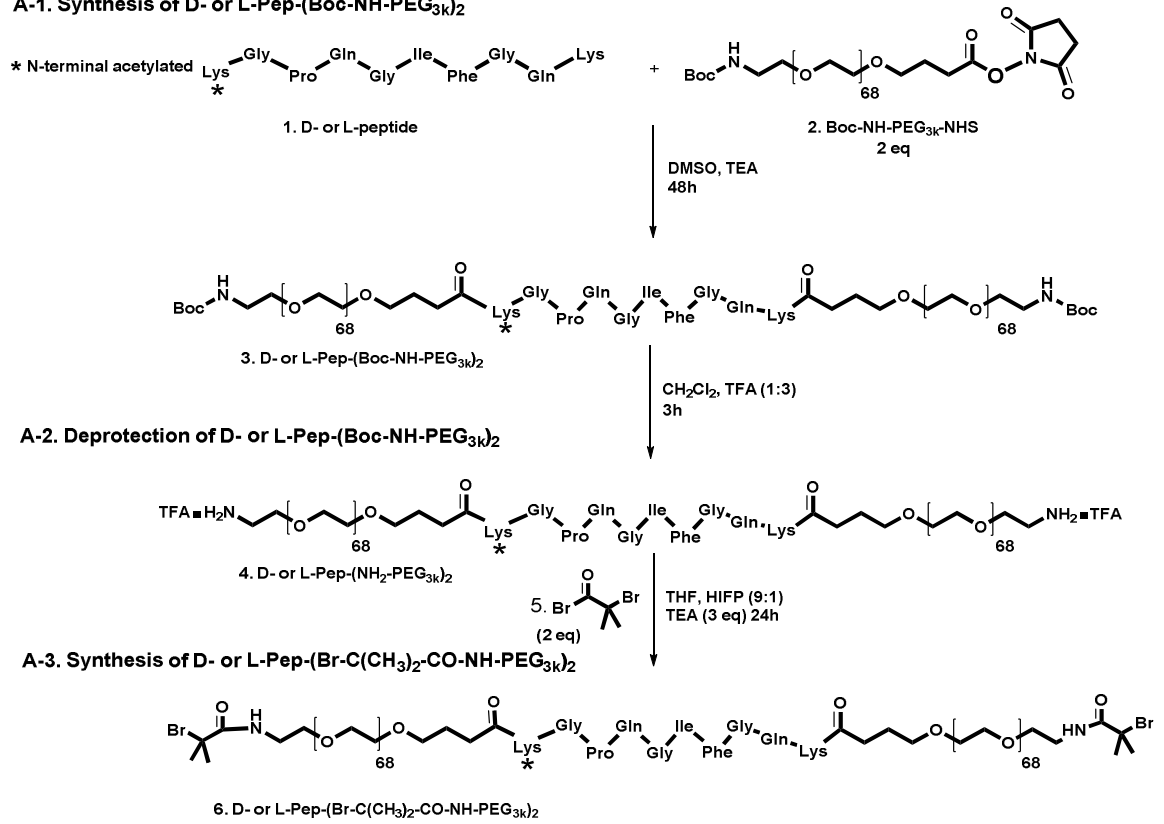

B. Synthesis of D or L-Pep-NC

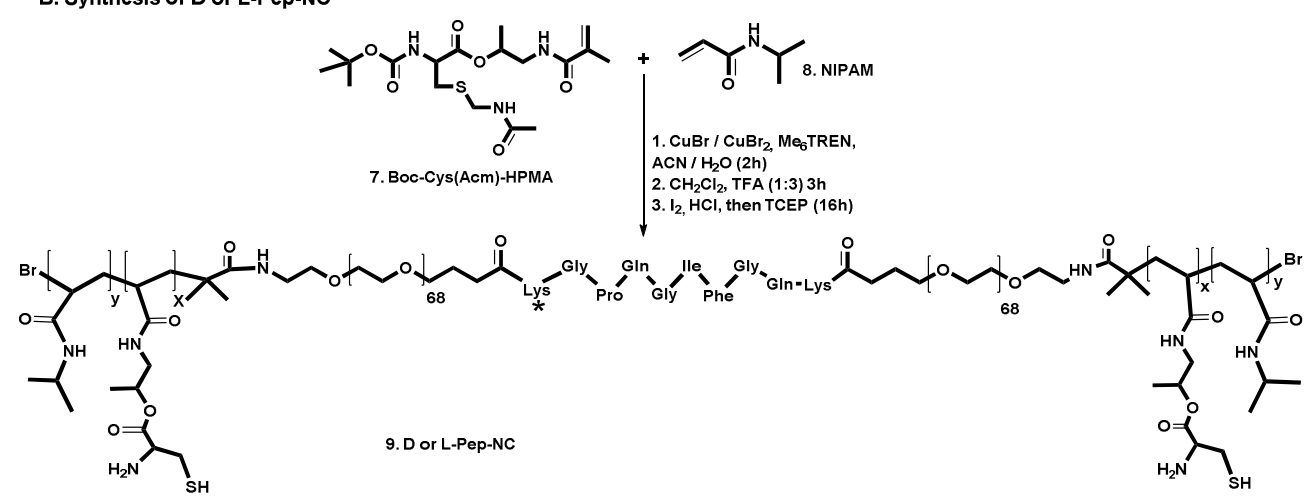

Figure 1: Synthesis route for A) D- or L-Pep-(Br-NH-PEG $\left.{ }_{3 k}\right)_{2}$ ATRP macroinitiator, B) ABA tri-block copolymer of D- or L-Pep-NC containing D- or L-Pep-(PEG $\left.G_{3 k}\right)_{2}$ as mid-blocks and copolymer of NIPAM and HPMA-Cys as outer-blocks. 


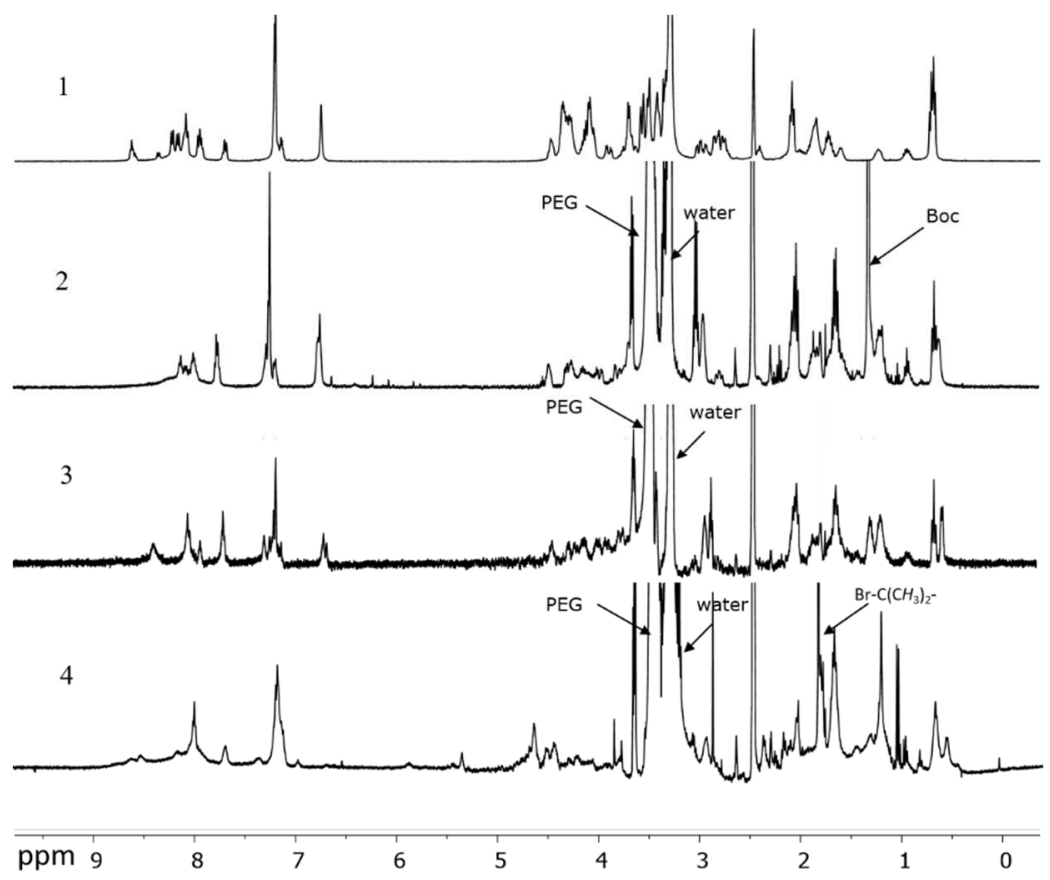

Figure 2: ${ }^{1} \mathrm{H}-\mathrm{NMR}$ spectra of the peptide Lys-Gly-Pro-Gln-Gly-Ile-Phe-Gly-Gln-Lys (1), peptide-PEG conjugated product with Boc protecting groups at the terminal ends (2), peptide-PEG conjugated polymers after Boc deprotection of the terminal amine groups (3), the ATRP peptide-PEG macroinitiator (4). Deuterated DMSO was used as solvent.

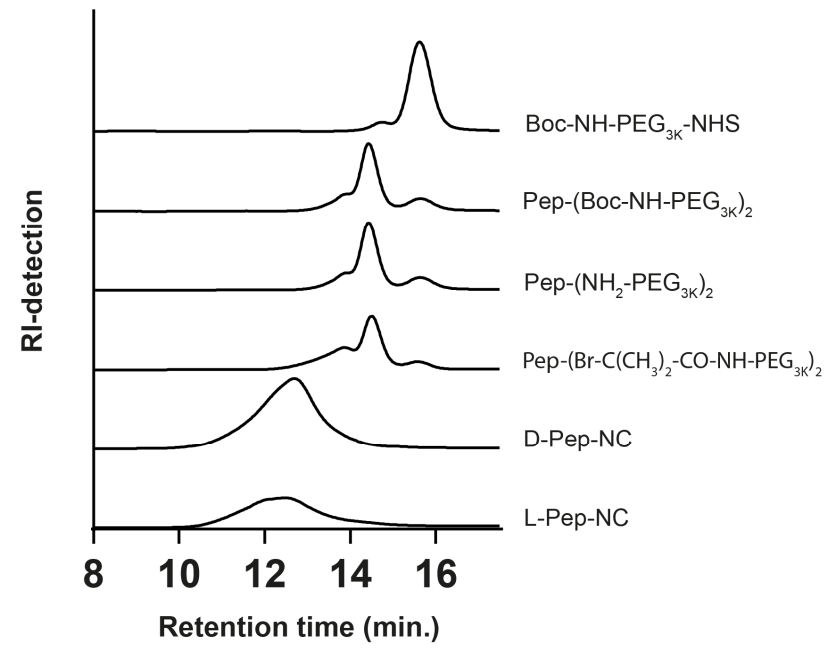

Figure 3: GPC chromatograms of starting compound (Boc-NH-PEG-NHS), peptide-conjugated products ((Boc-NH ${ }_{2}$-PEG $\left.3 \mathrm{kDa}\right)_{2}$-Pep and $\left(\mathrm{NH}_{2} \text {-PEG } 3 \mathrm{kDa}\right)_{2}$-Pep), ATRP peptide-PEG macroinitiator $\left(\left(\mathrm{Br}-\mathrm{C}\left(\mathrm{CH}_{3}\right)_{2}-\right.\right.$ CO-NH-PEG $3 \mathrm{kDa})_{2}-\mathrm{Pep}$ ), and final products (D-Pep-NC and L-Pep-NC). 


\subsubsection{Enzymatic cleavage of the thermosensitive ABCBA pentablock polymers} (Pep-NC)

The pentablock copolymer, Pep-NC, forms flower-like micelles in PBS with a Z-average of $87 \pm 1 \mathrm{~nm}$ (PDI 0.1) at temperatures above the lower critical solution temperature (LCST 31 ${ }^{\circ} \mathrm{C}$, table 1) (Fig. 4A). To study the accessibility of the peptide block in the polymer backbone for metalloproteases, D/L-Pep-NC polymers were incubated with metalloprotease type IV collagenase (a model for MMP type 2 and 9) above the LCST. Analysis of the obtained polymers by GPC showed that the molecular weight of D-Pep-NC did not change after incubation with collagenase while that of L-Pep-NC reduced to half its original molecular weight under the same conditions (SI-Fig. 12). These results demonstrate that the peptide sequence in L-Pep-NC in the micelles is indeed accessible for collagenase. As expected, cleavage of the peptide block in D-Pep-NC by the enzyme did not occur.

A
B

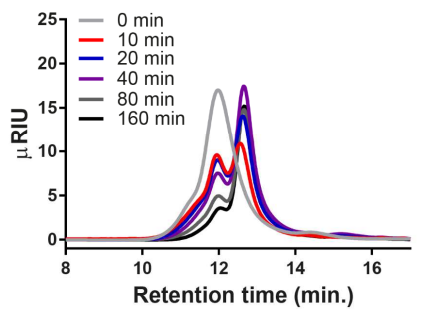

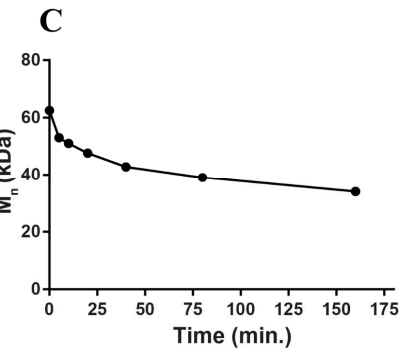

Figure 4: A) Formation of flower-like micelles from pentablock copolymer Pep-NC and cleavage of peptide midblock by collagenase. B) GPC chromatograms (IR detection) of L-Pep-NC incubated with collagenase ( 0.5 units. $\left.\mathrm{mL}^{-1}\right)$ at different times at $37^{\circ} \mathrm{C}$. C) $\mathrm{M}_{\mathrm{n}}$ of L-Pep-NC as a function of time as determined by GPC.

To investigate the kinetics of peptide cleavage of L-Pep-NC, the polymer was incubated with and without collagenase at $37^{\circ} \mathrm{C}$ and thus above the LCST of the polymer in PBS and samples were collected at different time points and analyzed by GPC (Fig. 4 B, C). No change in the molecular weight was observed in the absence of the enzyme even after $24 \mathrm{~h}$ of incubation (SI-Fig. 12). In contrast, in the presence of collagenase, a gradual increase in the retention time as a function of incubation time was observed. The change in the retention time (from 11.9 to $12.7 \mathrm{~min}$ ) corresponds to a decrease in $\mathrm{Mn}$ from 64 to $35 \mathrm{kDa}$ indicating cleavage of the peptide block within 160 minutes. The cleavage of the peptide at $37^{\circ} \mathrm{C}$ (above LCST of the Pep-NC) demonstrates the accessibility of the peptide block for the enzyme, even though other reported PNIPAM-peptide conjugates have shown the opposite ${ }^{56}$. The cleavage of the peptide in the L-Pep-NC pentablock copolymer can be explained by the 
presence of PEG as flanking blocks that force the peptide blocks to be exposed in the loops of hydrophilic PEG shells of the flower-like micelles formed above the LCST (Fig. 4A).

\subsection{Preparation of micellar hydrogel (HyMic), characterization, and degradation}

\subsubsection{Preparation of micellar hydrogel and characterization}

Preparation of HyMic was performed in two steps. In the first step, PNC and PNE polymer solutions were mixed followed by increasing the temperature above LCST of the polymers, which resulted in self-assembly of the polymers into flower-like micelles. The micellar core was crosslinked by native chemical ligation of the cysteine and thioester functionalities present in PNC (HPMA-Cys) and PNE (HPMA-ETSA), respectively ${ }^{37}$. The obtained micelles displayed a Z-average of $80 \pm 1 \mathrm{~nm}$ (PDI 0.09) and $\zeta$-potential of $-4.2 \pm 0.4$ at $37{ }^{\circ} \mathrm{C}$. Subsequently, the prepared micelles were purified by dialysis against water and then lyophilized without cryo-protectant. The slight increase in their size after lyophilization of micelles from $80 \pm 1$ to $93 \pm 2 \mathrm{~nm}$ (PDI: 0.1 ) indicates that aggregation occurred to a limited degree. The excess of PNE for the preparation of micelles resulted in reactive thioester functionalities in the micellar core, which can be used for bridging the micelles using PepNC linkers via native chemical ligation to yield a hydrogel network. In the second step, PepNC having free cysteine moieties was added to the CCL micelle dispersion with free thioester functionalities in the micellar cores at a temperature below the LCST of PNIPAM. At this temperature, Pep-NC and the polymeric chains in the core of the CCL micelles are swollen and consequently, Pep-NC molecules can diffuse into the hydrated core of the CCL micelles. The close proximity of thioester functionalities in the core of micelles to the cysteine functionalities in the Pep-NC linker facilitates native chemical ligation and triggers gel formation. Subsequent incubation of the CCL micelle-Pep-NC mixture above the LCST of the PNIPAM blocks resulted in a hydrogel, while the sample composed of only CCL micelles displayed a low viscous dispersion (Fig. 5A). The storage modulus of sample composed of only micelles remained constant (around $2 \mathrm{~Pa}$ ) during the entire experiment (SI-Fig. 13). In more detail, the CCL micelle-Pep-NC mixture displayed a G' of 600 Pa shortly after heating to $37^{\circ} \mathrm{C}$, which increased up to $1300 \mathrm{~Pa}$ after 160 minutes. A decrease in tan $\delta$ from 0.20 to 0.06 confirmed formation of a chemically crosslinked hydrogel. The relatively high $\mathrm{G}^{\prime}$ (and low $\tan \delta$ ) value of the CCL micelle-Pep-NC mixture at the start of the measurement can likely be attributed to crosslinking of the micelles that occurred during sample preparation and before the start of the measurement. Although the $\mathrm{G}^{\prime}$ is relatively high (600 Pa), $150 \mu \mathrm{L}$ of the CCL micelle-Pep-NC mixture was easily passed through a needle (gauge 23 ) at $37^{\circ} \mathrm{C}$, therefore, it can be considered as an injectable material at body temperature. This is in line 
with what has been shown before by Van Tomme et al. that hydrogels with $\mathrm{G}^{\prime}<4000 \mathrm{~Pa}$ are suitable for injection ${ }^{57}$. These results demonstrate the formation of a crosslinked network of micelles, which is further abbreviated as "HyMic" (Fig. 5A). The overall gel concentration can be adjusted according to the final application and the required gel stiffness. Stable gels can be formed at polymer concentrations above $12 \%$ and at least up to $30 \%$. The gel stiffness can be increased by increasing the total polymer concentration. However, the ratio between the cysteine functionalities in the linker and thioester functionalities in the micellar core should be considered for optimal crosslinking.

A
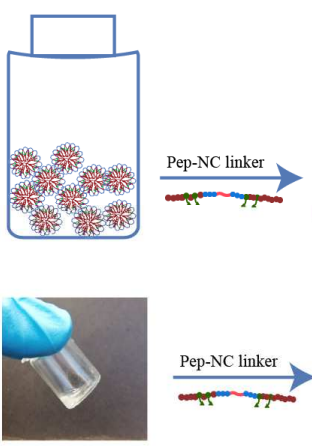

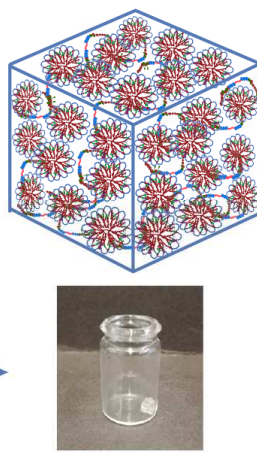

B

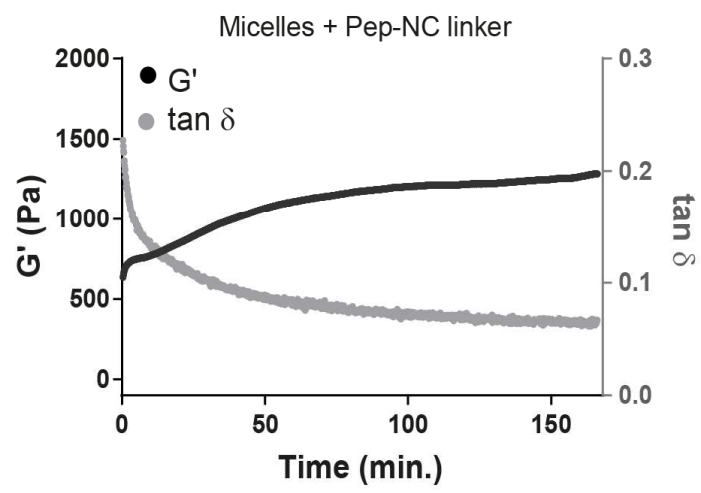

Figure 5: Formation of HyMic A) lyophilized core crosslinked flower-like micelles were dispersed in PBS and subsequently mixed with L-Pep-NC to form a hydrogel. B) Storage modulus (G') and $\tan \delta$ as a function of time for micelles after mixing with L-Pep-NC at $37^{\circ} \mathrm{C}$.

\subsubsection{Enzymatic degradation of HyMic}

As shown in section 3.1.3, the peptide midblock in the L-Pep-NC polymer can be cleaved by collagenase. To investigate the accessibility of L-Pep-NC in the gel structure and consequently enzyme responsivity of HyMic, the gel composed of CCL micelles and the LPep-NC (L-HyMic) was incubated with collagenase at different concentrations at $37^{\circ} \mathrm{C}$ (Fig. 6A). HyMic composed of CCL micelles and D-Pep-NC (D-HyMic) was only treated with the highest concentration of collagenase used for L-HyMic treatment (30 units. $\mathrm{mL}^{-1}$ ).

Both types of gels displayed a maximum swelling ratio of 2.0 and no gel erosion was observed in the absence of the enzyme. L-HyMic exhibited complete degradation in seven days at an enzyme concentration of 30 units. $\mathrm{mL}^{-1}$, while $\mathrm{D}$-HyMic remained intact even after 2 months at the same enzyme concentration (Fig. 6A), which demonstrates that degradation is indeed triggered by cleavage of L-Pep-NC linker. Interestingly, the 
degradation rate of L-HyMic was significantly slower than of L-Pep-NC (7 days vs. $160 \mathrm{~min}$ (Fig. 4)) even though, the substrate to enzyme ratio was 5 times lower (see section 3.1.3). This slower degradation of L-HyMic could be attributed to the limited accessibility of collagenase to the peptide block in the hydrogel network. The gel degradation as a function of enzyme concentration (Fig. 6) can reveal insights into the degradation mechanism. The observed degradation times were 45,21 , and 7 days, in the presence of $7.5,15.0$, and 30.0 units of enzyme per milliliter, respectively. The samples incubated with 30 units. $\mathrm{mL}^{-1}$ exhibited a weight loss after reaching maximum swelling while the gels incubated with 15 and 7.5 units. $\mathrm{mL}^{-1}$ of collagenase showed a constant gel weight for about 10 and 25 days, respectively (Fig. 6A). The plateau value in hydrogel weight can be ascribed to absorption of water due to a decreasing crosslink density of the hydrogel network, which is compensated by shedded particles. At the decaying point of the graph (Fig. 6A), (around day 10 and day 25 for gels incubated with 15 units. $\mathrm{mL}^{-1}$ and 7.5 units. $\mathrm{mL}^{-1}$ of collagenase, respectively) the gel network became very weak, resulting in rapid disintegration of the gels. Figure 6B shows that the degradation time of the gels decreased with increasing enzyme concentration. It is known that for surface erosion, the degradation rate is not affected by enzyme concentration above a certain concentration due to saturation of the surface with enzyme molecules. On the other hand, for bulk degradation the degradation rate increases with increasing enzyme concentration ${ }^{58,59}$. Estimation of hydrogel mesh size $(\xi)$ based on rubber elasticity theory ${ }^{60,61}$ (equation in section 2.4.3) showed a mesh size of $\sim 7 \mathrm{~nm}$ for the gel with a $\mathrm{G}^{\prime}$ of $1300 \mathrm{~Pa}$. This means that collagenase with a molecular weight of 63-130 $\mathrm{kDa}^{62,63}\left(\mathrm{R}_{\mathrm{h}} \sim 3.5-4.5 \mathrm{~nm}\right)^{64}$ can penetrate into the hydrogel network and initiate bulk degradation. Therefore, the observed degradation is very likely due to combination of bulk degradation and surface erosion.

The release medium of the L-HyMic and D-HyMic hydrogels incubated with 30 units. $\mathrm{mL}^{-1}$ of collagenase was refreshed daily and analyzed using DLS. Additionally, the fluorescence intensity of the release medium was measured to determine the concentration of the released dye-conjugated CCL micelles (Fig. 7). The D-HyMic release medium only exhibited a detectable signal for the derived count rate on the first day, which was much lower than the recorded value for L-HyMic (1800 vs. 48000). From day 2 on, no signal above background was recorded and thus no nanoparticles were present in the D-HyMic release medium (Fig. $7 \mathrm{~B}$ ). The released particles at day one exhibited a Z-average of $100 \mathrm{~nm}$ and PDI of 0.1 suggesting the release of intact $\mathrm{CCL}$ micelles (Fig. 7A, C). Measuring the concentration of the released dye-conjugated $C C L$ micelles using fluorescence showed $10 \%$ release of the CCL micelles on the first day (Fig. 7D). Taken together, during the first day CCL micelles were released from the $\mathrm{D}-\mathrm{HyMic}$ that were not connected to the gel network. Clearly, collagenase is unable to cleave D-Pep-NC to result in shedding of CCL micelles over time. 


\section{A}

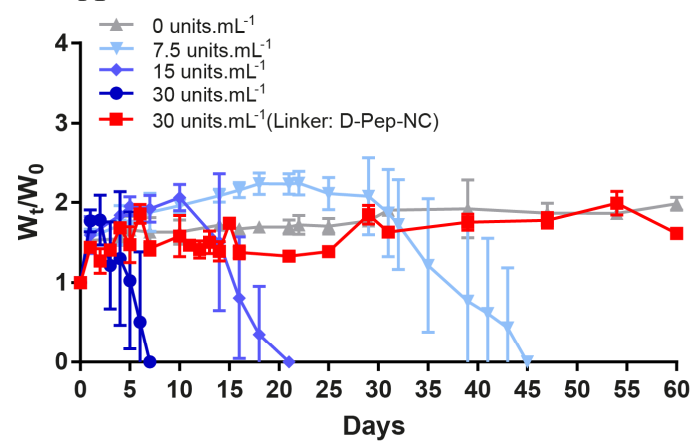

B

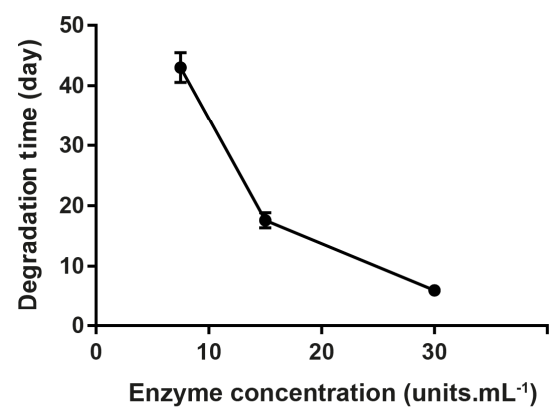

Figure 6: A) Enzymatic degradation of L-HyMic at different concentrations of collagenase. B) Degradation time as a function of enzyme concentration at $37^{\circ} \mathrm{C}$ and $\mathrm{pH}$ 7.4. Degradation time is reported as the recorded time for full degradation of a gel $(n=3)$.

In contrast to D-HyMic, the release medium of L-HyMic exhibited high values for the derived count rate in the first days, which decreased over time (Fig. 7B). The released particles displayed size of $\sim 110$ (PDI $\sim 0.2$ ) which raised to $\sim 200 \mathrm{~nm}$ (PDI $\sim 0.3$ ) from day 2 on. The high derived count rate values in the first days can be explained by the release of $\mathrm{CCL}$ micelles on the surface, which may have a low number of connections to the micellar network. The latter can also explain the similar size and PDI of the released particles compared with lyophilized micelles that were used for L-HyMic formation (Fig 7A). The bigger size and PDI after the first day indicate release of nanosized gel fragments composed of more than one CCL micelle (Fig. 7A). Measuring the fluorescence intensity of the released dye-conjugated CCL micelles showed that approximately $50 \%$ of the $C C L$ micelles were released upon gel exposure to 30 unit. $\mathrm{mL}^{-1}$ collagenase at day one and the $\mathrm{CCL}$ micelle release was completed after seven days. This is in line with the high value of the derived count rated on the first day (Fig. 7B), low swelling ratio, and immediate weight loss of this gel (Fig. 6A). Fluorescence imaging of L-HyMic and D-HyMic hydrogels and their release medium was performed after 2 and 30 days incubation with collagenase (Fig. 7 E, F). As expected, a strong fluorescent signal was detected in the release medium of L-HyMic while the signal in D-HyMic release medium was below the detection limit. Interestingly, fluorescence imaging showed a fluffy structure for the L-HyMic gel while the D-HyMic displayed a dense structure even after one month incubation with collagenase. This fluffy swollen structure is most likely due to cleavage of Pep-NC linkers inside the gel network due to diffusion of the enzyme in the hydrogel matrix as mentioned above. This observation supports that degradation of the 
hydrogel in the presence of collagenase is due to a combination of surface erosion and bulk degradation. Such a micellar hydrogel could be injected in tumor tissues. The upregulation of MMPs in tumor tissues would then trigger the gel degradation resulting in the release of micelles. When micelles are loaded with anti-cancer therapeutics the internalization of the released micelles in cancer cells can cause cytotoxicity and consequently tumor regression.

A Micelles before lyophilization $\quad \boldsymbol{\nabla}$ Micelles after lyophilization

- Linker: L-Pep-NC a Linker: D-Pep-NC
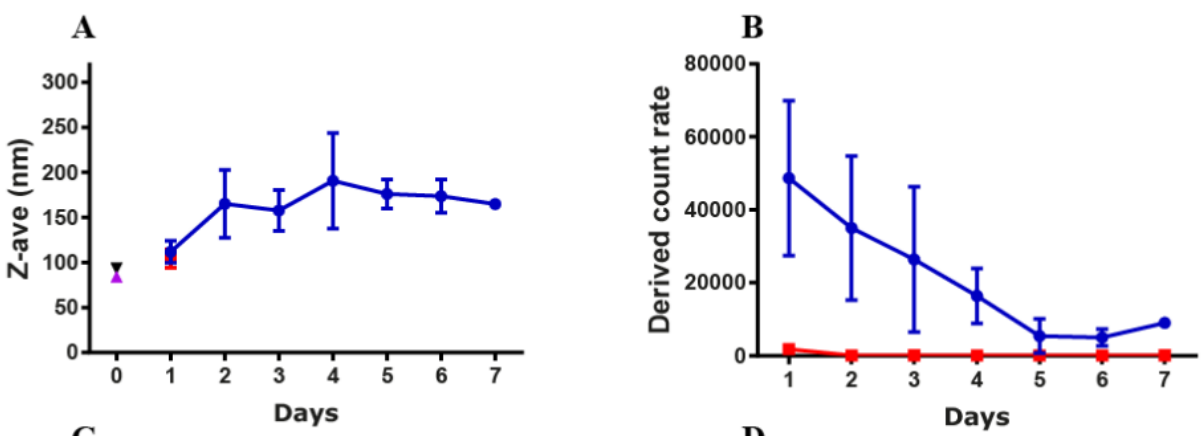

C

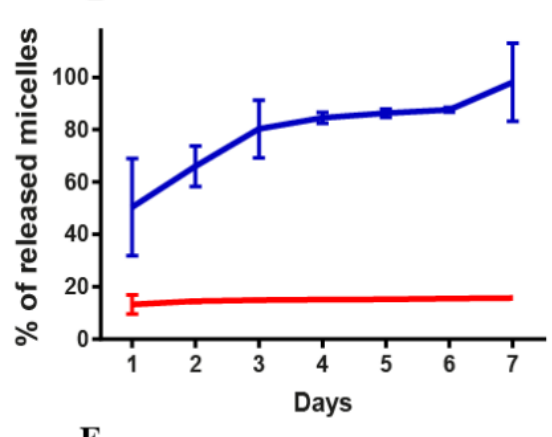

$\mathbf{E}$

$\mathbf{F}$

Release medium

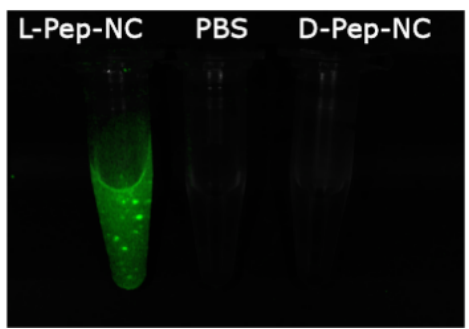

HyMic

L-Pep-NC PBS D-Pep-NC

es

4

Figure 7: DLS analysis of the gel medium of $L$ and D-HyMic upon incubation with collagenase, A) Zaverage $B$ ) derived count rate, C) PDI, D) the percentage of the released dye-conjugated micelles from HyMic ( $n=3)$. Fluorescence imaging of $E$ ) the release medium and F) HyMic hydrogels incubated with

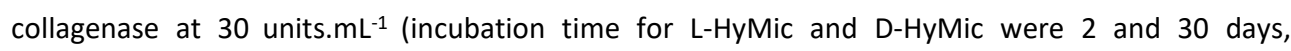
respectively). 


\subsection{Cellular internalization of CCL micelles by HeLa cells}

The suitability of the released CCL upon enzymatic degradation of L-HyMic for intracellular drug delivery was investigated using HeLa cells. To this end, dye-conjugated CCL micelles were formulated into HyMic using L-Pep-NC and subsequently incubated with collagenase. The concentration of the released CCL micelles was monitored by measuring the dye concentration in the release medium. The released CCL micelles obtained after 21 days of incubation with collagenase displayed a Z-average of $120 \pm 2 \mathrm{~nm}$ (PDI 0.2) and $\zeta$-potential of $-2.7 \pm 0.0$ at $37^{\circ} \mathrm{C}$. The confocal images (Fig. 8) showed punctate fluorescence confirming the internalization of both control micelles and released CCL micelles after $24 \mathrm{~h}$ incubation with the cells. The uptake of released CCL micelles from L-HyMic upon its enzymatic degradation shows the potential of enzymatic cleavable L-HyMic for intracellular drug delivery.

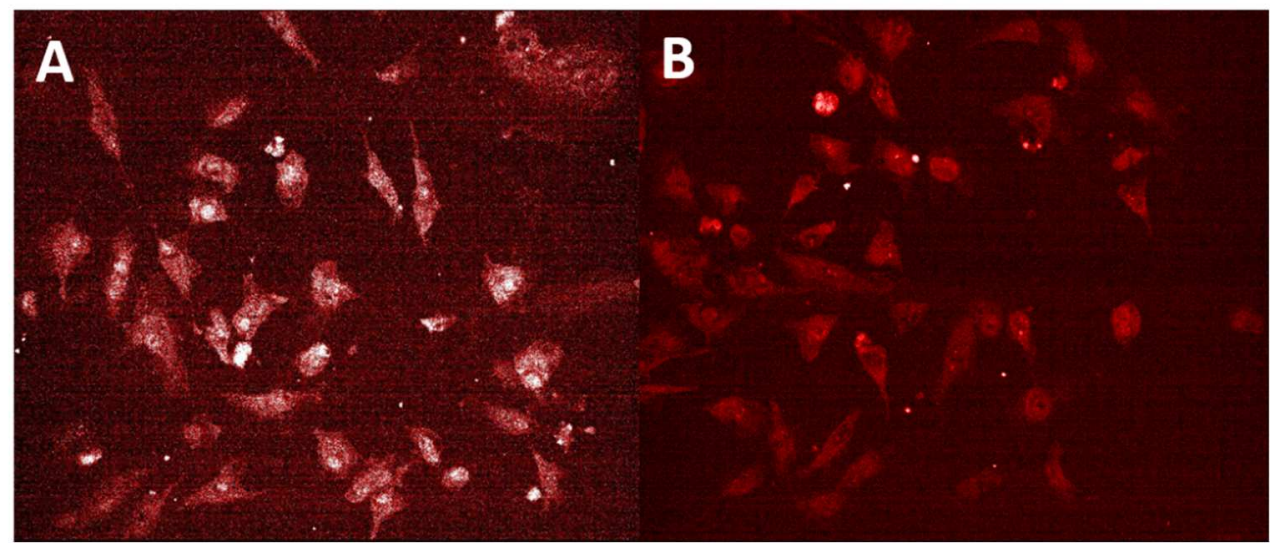

Figure 8: Internalization of freshly prepared micelles and micelles released from the L-HyMic hydrogel upon enzymatic degradation. Laser confocal scanning microscopy of HeLa cells incubated for $24 \mathrm{~h}$ with (A) freshly prepared fluorescently labeled CCL micelles at a concentration of $400 \mu \mathrm{g} \cdot \mathrm{mL}^{-1}$, (B) CCL micelles released from the gel at a concentration of $400 \mu \mathrm{g} \cdot \mathrm{mL}^{-1}$. Micelles are visualized with maleimide-Alexa fluor C5 568 in red.

\section{Conclusion}

This paper describes the design and synthesis of an enzyme responsive hydrogel (HyMic) consisting of $\mathrm{CCL}$ flower-like micelles and an enzyme responsive linker (Pep-NC). The complete degradation of HyMic in the presence of collagenase in a concentration dependent manner shows the programmability of this hydrogel. Upon enzymatic degradation, HyMic is converted into CCL micelles that can be taken up by HeLa cells. These 
results demonstrate the great potential of HyMic for sustained release of CCL micelles for intracellular drug delivery in tissues with upregulation of MMP e.g. cancer tissue. The introduced micellar hydrogel technology can be easily used for the development of other types of enzyme responsive micellar hydrogels. To this end, the peptide block in the linker can be substituted by a peptide that matches the specificity of the desired enzyme. Moreover, micelles can be decorated with targeting ligand to improve their cellular uptake upon release from the hydrogel.

\section{Acknowledgments}

The Netherlands Organization for Scientific Research (NWO/Aspasia 015.009.038 and NWO/VIDI 13457) is acknowledged for funding. 


\section{References}

1. Hoffman, A. S., Hydrogels for biomedical applications. Advanced Drug Delivery Reviews 2012, 64, 18-23.

2. Buwalda, S. J.; Boere, K. W. M.; Dijkstra, P. J.; Feijen, J.; Vermonden, T.; Hennink, W. E., Hydrogels in a historical perspective: From simple networks to smart materials. Journal of Controlled Release 2014, 190, 254-273.

3. Hoare, T. R.; Kohane, D. S., hydrogels in drug delivery:progress and challenges. Polymer 2008, 49, 1993-2007.

4. Vermonden, T.; Censi, R.; Hennink, W. E., Hydrogels for protein delivery. Chemical Reviews 2012, 112 (5), 2853-88.

5. Peppas, N. A.; Wood, K. M.; Blanchette, J. O., Hydrogels for oral delivery of therapeutic proteins. Expert Opinion on Biological Therapy 2004, 4 (6), 881-887.

6. Fliervoet, L. A. L.; Engbersen, J. F. J.; Schiffelers, R. M.; Hennink, W. E.; Vermonden, T., Polymers and hydrogels for local nucleic acid delivery. Journal of Materials Chemistry $B$ 2018, 6 (36), 5651-5670.

7. Dosio, F.; Arpicco, S.; Stella, B.; Fattal, E., Hyaluronic acid for anticancer drug and nucleic acid delivery. Advanced Drug Delivery Reviews 2016, 97, 204-236.

8. Buwalda, S. J.; Vermonden, T.; Hennink, W. E., Hydrogels for Therapeutic Delivery: Current Developments and Future Directions. Biomacromolecules 2017, 18 (2), 316-330.

9. McKenzie, M.; Betts, D.; Suh, A.; Bui, K.; Kim, D. L.; Cho, H., Hydrogel-Based Drug Delivery Systems for Poorly Water-Soluble Drugs. Molecules 2015, 20 (11).

10. Gou, M.; Li, X.; Dai, M.; Gong, C.; Wang, X.; Xie, Y.; Deng, H.; Chen, L.; Zhao, X.; Qian, Z.; Wei, Y., A novel injectable local hydrophobic drug delivery system: Biodegradable nanoparticles in thermo-sensitive hydrogel. International Journal of Pharmaceutics 2008, 359 (1), 228-233.

11. O'Neill, H. S.; Herron, C. C.; Hastings, C. L.; Deckers, R.; Lopez Noriega, A.; Kelly, H. M.; Hennink, W. E.; McDonnell, C. O.; O’Brien, F. J.; Ruiz-Hernández, E.; Duffy, G. P., A stimuli responsive liposome loaded hydrogel provides flexible on-demand release of therapeutic agents. Acta Biomaterialia 2017, 48, 110-119.

12. Stenekes, R. J. H.; Loebis, A. E.; Fernandes, C. M.; Crommelin, D. J. A.; Hennink, W. E., Controlled Release of Liposomes from Biodegradable Dextran Microspheres: A Novel Delivery Concept. Pharmaceutical Research 2000, 17 (6), 664-669.

13. Wei, L.; Cai, C.; Lin, J.; Chen, T., Dual-drug delivery system based on hydrogel/micelle composites. Biomaterials 2009, 30 (13), 2606-13.

14. Li, J.; Mooney, D. J., Designing hydrogels for controlled drug delivery. Nature Reviews Materials 2016, 1, 16071. 
15. Eljarrat-Binstock, E.; Orucov, F.; Aldouby, Y.; Frucht-Pery, J.; Domb, A. J., Charged nanoparticles delivery to the eye using hydrogel iontophoresis. Journal of Controlled Release 2008, 126 (2), 156-161.

16. Henke, M.; Brandl, F.; Goepferich, A. M.; Tessmar, J. K., Size-dependent release of fluorescent macromolecules and nanoparticles from radically cross-linked hydrogels. European Journal of Pharmaceutics and Biopharmaceutics 2010, 74 (2), 184-192.

17. de Graaf, A. J.; Azevedo Prospero dos, S., II; Pieters, E. H.; Rijkers, D. T.; van Nostrum, C. F.; Vermonden, T.; Kok, R. J.; Hennink, W. E.; Mastrobattista, E., A micelle-shedding thermosensitive hydrogel as sustained release formulation. Journal of Controlled Release 2012, 162 (3), 582-90.

18. Qiu, S.; Zhuang, J.; Jin, S.; Yang, N.-L., Nitrocatecholic copolymers - synthesis and their remarkable binding affinity. Chemical Communications 2019, 55 (72), 10748-10751.

19. Xiao, L.; Zhu, J.; Londono, J. D.; Pochan, D. J.; Jia, X., Mechano-responsive hydrogels crosslinked by block copolymer micelles. Soft Matter 2012, 8 (40), 10233-10237.

20. Ghoorchian, A.; Simon, J. R.; Bharti, B.; Han, W.; Zhao, X.; Chilkoti, A.; López, G. P., Bioinspired Reversibly Cross-linked Hydrogels Comprising Polypeptide Micelles Exhibit Enhanced Mechanical Properties. Advanced Functional Materials 2015, 25 (21), 3122-3130.

21. Guillet, P.; Mugemana, C.; Stadler, F. J.; Schubert, U. S.; Fustin, C.-A.; Bailly, C.; Gohy, J.-F., Connecting micelles by metallo-supramolecular interactions: towards stimuli responsive hierarchical materials. Soft Matter 2009, 5 (18), 3409-3411.

22. Hoffman, A. S., Stimuli-responsive polymers: Biomedical applications and challenges for clinical translation. Advanced Drug Delivery Reviews 2013, 65 (1), 10-16.

23. Soppimath, K. S.; Aminabhavi, T. M.; Dave, A. M.; Kumbar, S. G.; Rudzinski, W. E., Stimulus-Responsive "Smart" Hydrogels as Novel Drug Delivery Systems. Drug Development and Industrial Pharmacy 2002, 28 (8), 957-974.

24. Dadsetan, M.; Liu, Z.; Pumberger, M.; Giraldo, C. V.; Ruesink, T.; Lu, L.; Yaszemski, M. J., A stimuli-responsive hydrogel for doxorubicin delivery. Biomaterials 2010, 31 (31), 80518062.

25. Peng, K.; Tomatsu, I.; Kros, A., Light controlled protein release from a supramolecular hydrogel. Chemical Communications 2010, 46 (23), 4094-4096.

26. Najafi, M.; Hebels, E.; Hennink, W. E.; Vermonden, T., Poly( N -isopropylacrylamide): Physicochemical Properties and Biomedical Applications: Temperature-Responsive Polymers: Chemistry, Properties, and Applications, Khutoryanskiy, V. V.; Georgiou, K. T., Eds. John Wiley \& Sons: 2018; pp 1-34.

27. Qiu, Y.; Park, K., Environment-sensitive hydrogels for drug delivery. Advanced Drug Delivery Reviews 2001, 53 (3), 321-339. 
28. West, J. L.; Hubbell, J. A., Polymeric Biomaterials with Degradation Sites for Proteases Involved in Cell Migration. Macromolecules 1999, 32 (1), 241-244.

29. Chandrawati, R., Enzyme-responsive polymer hydrogels for therapeutic delivery. Experimental Biology and Medicine 2016, 241 (9), 972-979.

30. Ooi, H. W.; Hafeez, S.; van Blitterswijk, C. A.; Moroni, L.; Baker, M. B., Hydrogels that listen to cells: a review of cell-responsive strategies in biomaterial design for tissue regeneration. Materials Horizons 2017, 4 (6), 1020-1040.

31. Purcell, B. P.; Lobb, D.; Charati, M. B.; Dorsey, S. M.; Wade, R. J.; Zellars, K. N.; Doviak, H.; Pettaway, S.; Logdon, C. B.; Shuman, J. A.; Freels, P. D.; Gorman lii, J. H.; Gorman, R. C.; Spinale, F. G.; Burdick, J. A., Injectable and bioresponsive hydrogels for on-demand matrix metalloproteinase inhibition. Nature Materials 2014, 13, 653.

32. Hesse, E.; Freudenberg, U.; Niemietz, T.; Greth, C.; Weisser, M.; Hagmann, S.; Binner, M.; Werner, C.; Richter, W., Peptide-functionalized starPEG/heparin hydrogels direct mitogenicity, cell morphology and cartilage matrix distribution in vitro and in vivo. Journal of Tissue Engineering and Regenerative Medicine 2018, 12 (1), 229-239.

33. Westermarck, J.; KÄHÄRi, V.-M., Regulation of matrix metalloproteinase expression in tumor invasion. The FASEB Journal 1999, 13 (8), 781-792.

34. Foda, H. D.; Zucker, S., Matrix metalloproteinases in cancer invasion, metastasis and angiogenesis. Drug Discovery Today 2001, 6 (9), 478-482.

35. Roy, R.; Yang, J.; Moses, M. A., Matrix metalloproteinases as novel biomarkers and potential therapeutic targets in human cancer. Journal of clinical oncology 2009, 27 (31), 5287-5297.

36. Egeblad, M.; Werb, Z., New functions for the matrix metalloproteinases in cancer progression. Nature Reviews Cancer 2002, 2 (3), 161-174.

37. Najafi, M.; Kordalivand, N.; Moradi, M. A.; van den Dikkenberg, J.; Fokkink, R.; Friedrich, H.; Sommerdijk, N.; Hembury, M.; Vermonden, T., Native Chemical Ligation for Cross-Linking of Flower-Like Micelles. Biomacromolecules 2018, 19 (9), 3766-3775.

38. Netzel-Arnett, S.; Sang, Q. X.; Moore, W. G. I.; Navre, M.; Birkedal-Hansen, H.; Van Wart, H. E., Comparative sequence specificities of human 72- and 92-kDa gelatinases (type IV collagenases) and PUMP (matrilysin). Biochemistry 1993, 32 (25), 6427-6432.

39. Ulbrich, K.; Šubr, V.; Strohalm, J.; Plocová, D.; Jelínková, M.; Říhová, B., Polymeric drugs based on conjugates of synthetic and natural macromolecules: I. Synthesis and physico-chemical characterisation. Journal of Controlled Release 2000, 64 (1), 63-79.

40. Boere, K. W. M.; Soliman, B. G.; Rijkers, D. T. S.; Hennink, W. E.; Vermonden, T., Thermoresponsive Injectable Hydrogels Cross-Linked by Native Chemical Ligation. Macromolecules 2014, 47 (7), 2430-2438. 
41. de Graaf, A. J.; Mastrobattista, E.; Vermonden, T.; van Nostrum, C. F.; Rijkers, D. T. S.; Liskamp, R. M. J.; Hennink, W. E., Thermosensitive Peptide-Hybrid ABC Block Copolymers Obtained by ATRP: Synthesis, Self-Assembly, and Enzymatic Degradation. Macromolecules 2012, 45 (2), 842-851.

42. Boere, K. W.; van den Dikkenberg, J.; Gao, Y.; Visser, J.; Hennink, W. E.; Vermonden, T., Thermogelling and Chemoselectively Cross-Linked Hydrogels with Controlled Mechanical Properties and Degradation Behavior. Biomacromolecules 2015, 16 (9), 2840-51.

43. Soga, O.; van Nostrum, C. F.; Ramzi, A.; Visser, T.; Soulimani, F.; Frederik, P. M.; Bomans, P. H. H.; Hennink, W. E., Physicochemical Characterization of Degradable Thermosensitive Polymeric Micelles. Langmuir 2004, 20 (21), 9388-9395.

44. Tsurkan, M. V.; Chwalek, K.; Levental, K. R.; Freudenberg, U.; Werner, C., Modular StarPEG-Heparin Gels with Bifunctional Peptide Linkers. Macromolecular Rapid Communications 2010, 31 (17), 1529-1533.

45. Wu, Z.-S.; Wu, Q.; Yang, J.-H.; Wang, H.-Q.; Ding, X.-D.; Yang, F.; Xu, X.-C., Prognostic significance of MMP-9 and TIMP-1 serum and tissue expression in breast cancer. International Journal of Cancer 2008, 122 (9), 2050-2056.

46. Ando, H.; Twining, S. S.; Yue, B. Y.; Zhou, X.; Fini, M. E.; Kaiya, T.; Higginbotham, E. J.; Sugar, J., MMPs and proteinase inhibitors in the human aqueous humor. Investigative Ophthalmology \& Visual Science 1993, 34 (13), 3541-3548.

47. Thompson, J. M.; Agee, K.; Sidow, S. J.; McNally, K.; Lindsey, K.; Borke, J.; Elsalanty, M.; Tay, F. R.; Pashley, D. H., Inhibition of Endogenous Dentin Matrix Metalloproteinases by Ethylenediaminetetraacetic Acid. Journal of Endodontics 2012, 38 (1), 62-65.

48. Matyjaszewski, K.; Xia, J., Atom Transfer Radical Polymerization. Chemical Reviews 2001, 101 (9), 2921-2990.

49. Isidro-Llobet, A.; Álvarez, M.; Albericio, F., Amino Acid-Protecting Groups. Chemical Reviews 2009, 109 (6), 2455-2504.

50. Dawson, P. E.; Muir, T. W.; Clark-Lewis, I.; Kent, S. B., Synthesis of proteins by native chemical ligation. Science 1994, 266 (5186), 776.

51. Smithenry, D. W.; Kang, M.-S.; Gupta, V. K., Telechelic Poly(N-isopropylacrylamide): Polymerization and Chain Aggregation in Solution. Macromolecules 2001, 34 (24), 8503 8511.

52. Ganachaud, F.; Monteiro, M. J.; Gilbert, R. G.; Dourges, M.-A.; Thang, S. H.; Rizzardo, E., Molecular Weight Characterization of Poly(N-isopropylacrylamide) Prepared by Living Free-Radical Polymerization. Macromolecules 2000, 33 (18), 6738-6745.

53. Blondelle, S. E.; Houghten, R. A., Comparison of 55\% TFA/CH2Cl2 and $100 \%$ TFA for Boc group removal during solid-phase peptide synthesis. International Journal of Peptide and Protein Research 1993, 41 (6), 522-527. 
54. Kamber, B.; Hartmann, A.; Eisler, K.; Riniker, B.; Rink, H.; Sieber, P.; Rittel, W., The Synthesis of Cystine Peptides by lodine Oxidation of S-Trityl-cysteine and SAcetamidomethyl-cysteine Peptides. Helvetica Chimica Acta 1980, 63 (4), 899-915.

55. Trzebicka, B.; Robak, B.; Trzcinska, R.; Szweda, D.; Suder, P.; Silberring, J.; Dworak, A., Thermosensitive PNIPAM-peptide conjugate - Synthesis and aggregation. European Polymer Journal 2013, 49 (2), 499-509.

56. Molawi, K.; Studer, A., Reversible switching of substrate activity of poly-Nisopropylacrylamide peptide conjugates. Chemical Communications 2007, (48), 5173-5175.

57. Van Tomme, S. R.; van Nostrum, C. F.; Dijkstra, M.; De Smedt, S. C.; Hennink, W. E., Effect of particle size and charge on the network properties of microsphere-based hydrogels. Eur. J. Pharm. Biopharm. 2008, 70, (2), 522-530.

58. Tsuji, H.; Ishida, T., Poly(L-lactide). X. Enhanced surface hydrophilicity and chainscission mechanisms of poly(L-lactide) film in enzymatic, alkaline, and phosphate-buffered solutions. Journal of Applied Polymer Science 2003, 87 (10), 1628-1633.

59. Wachiralarpphaithoon, C.; Iwasaki, Y.; Akiyoshi, K., Enzyme-degradable phosphorylcholine porous hydrogels cross-linked with polyphosphoesters for cell matrices. Biomaterials 2007, 28 (6), 984-993.

60. Rubinstein, M.; Colby, R. H., Polymer physics. Oxford University Press: Oxford; New York, 2003.

61. Atallah, P.; Schirmer, L.; Tsurkan, M.; Putra Limasale, Y. D.; Zimmermann, R.; Werner, C.; Freudenberg, U., In situ-forming, cell-instructive hydrogels based on glycosaminoglycans with varied sulfation patterns. Biomaterials 2018, 181, 227-239.

62. Bond, M. D.; Van Wart, H. E., Purification and separation of individual collagenases of Clostridium histolyticum using red dye ligand chromatography. Biochemistry 1984, 23 (13), 3077-3085.

63. Matsushita, O.; Jung, C.-M.; Katayama, S.; Minami, J.; Takahashi, Y.; Okabe, A., Gene Duplication and Multiplicity of Collagenases in Clostridium histolyticum. Journal of Bacteriology 1999, 181 (3), 923.

64. Erickson, H. P., Size and shape of protein molecules at the nanometer level determined by sedimentation, gel filtration, and electron microscopy. Biol Proced Online 2009, 11, 32-51. 


\section{Supporting information}

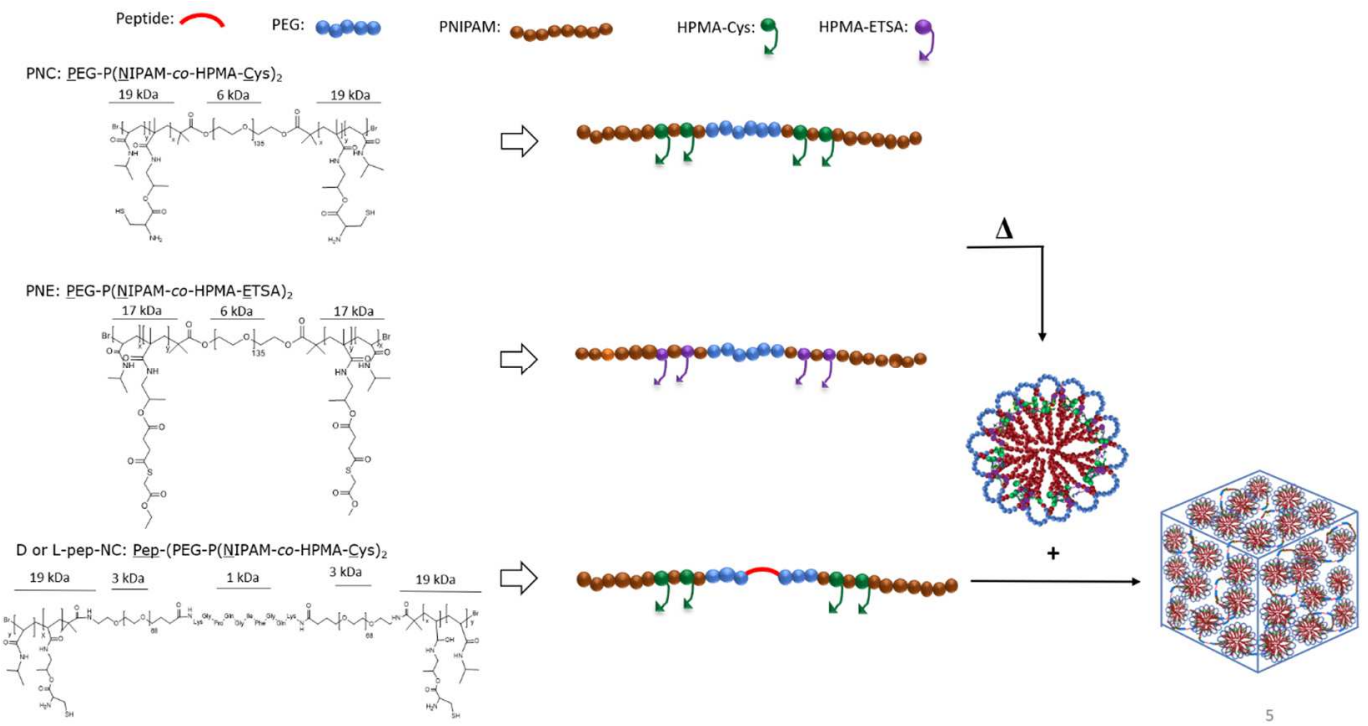

SI-figure 1: The structures of PNC, PNE, and Pep-NC. Core crosslinked flower-like micelles made from PNC and PNE linked via Pep-NC to yield a macroscopic hydrogel based on CCL crosslinked with an enzymatically cleavable spacer. 

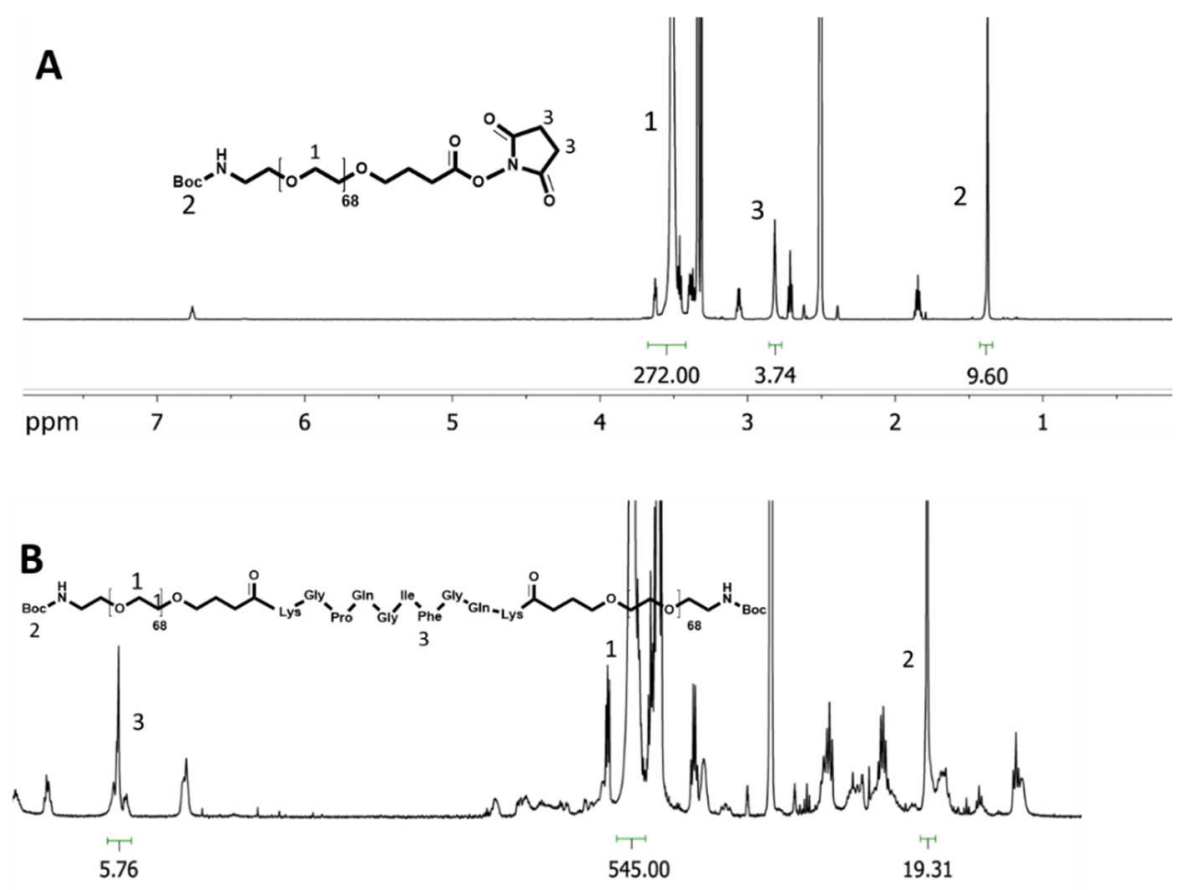

\begin{tabular}{lllllllll}
\hline $\mathrm{ppm}$ & 7 & 6 & 5 & 4 & 3 & 2 & 1
\end{tabular}

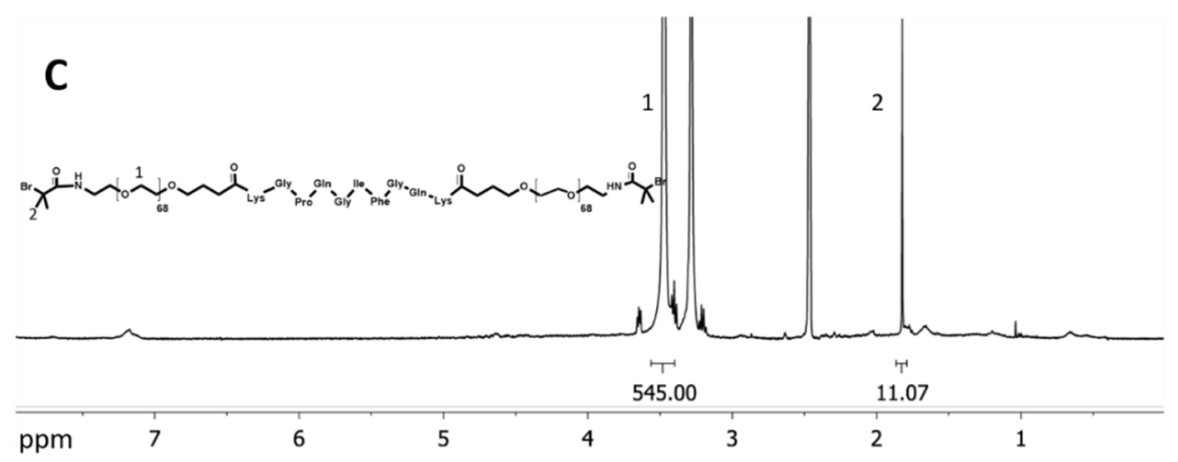

SI-Figure 2: ${ }^{1} \mathrm{H}-\mathrm{NMR}$ spectra of A) starting material of alpha-t-butyloxycarbonylamino- $\omega$-carboxy succinimidyl ester poly(ethylene glycol) (Boc-NH-PEG-NHS) (PEG-M 3 kDa), B) L-Pep-PEG conjugate before deprotection of boc group, C) L-Pep-PEG ATRP macroinitiator. The integrations are normalized based on the number of hydrogens in the corresponding PEG molecules. Deuterated DMSO was used as solvent. 


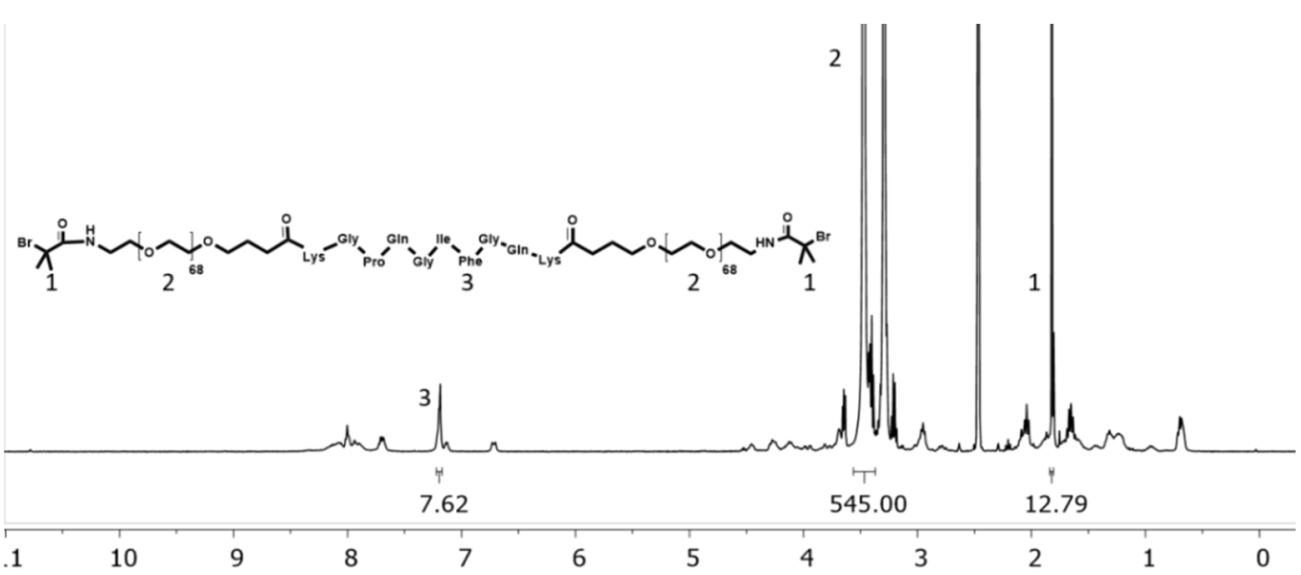

SI-Figure 3: ${ }^{1} \mathrm{H}-\mathrm{NMR}$ spectrum of D-Pep-PEG ATRP macroinitiator. The integrations are normalized based on the number of hydrogens in the corresponding PEG molecules. Deuterated DMSO was used as solvent.

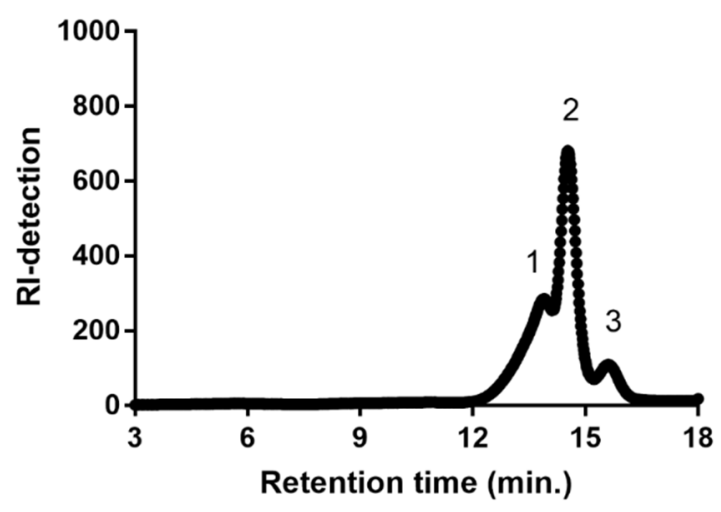

SI-Figure 4: GPC chromatogram of D-Pep-PEG ATRP macroinitiator in DMF containing $10 \mathrm{mM} \mathrm{LiCl.}$ Peak 1 corresponds to conjugation of peptide to the higher $M_{n}$ PEG derivative present in the commercial starting compound 2 (see Fig. 1.), peak 2 confirms formation of PEG-D-Pep-PEG with a $M_{n}$ of $7.9 \mathrm{kDa}$, and peak 3 belongs to non-conjugated PEG ( $\left.\mathrm{M}_{\mathrm{n}}: 3.4 \mathrm{kDa}\right)$. 
A

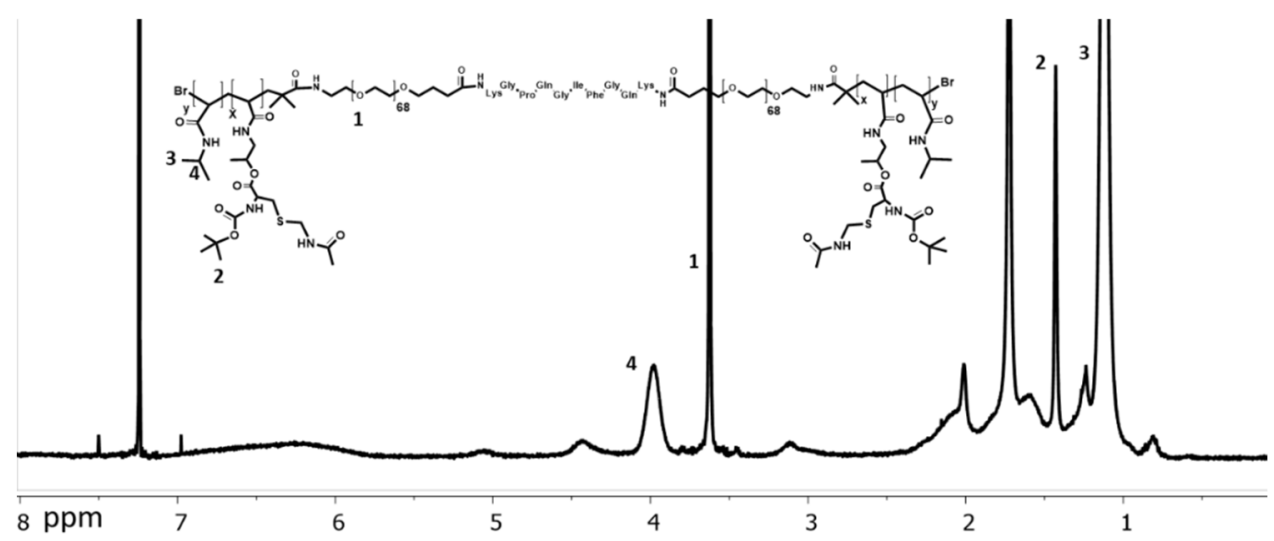

B
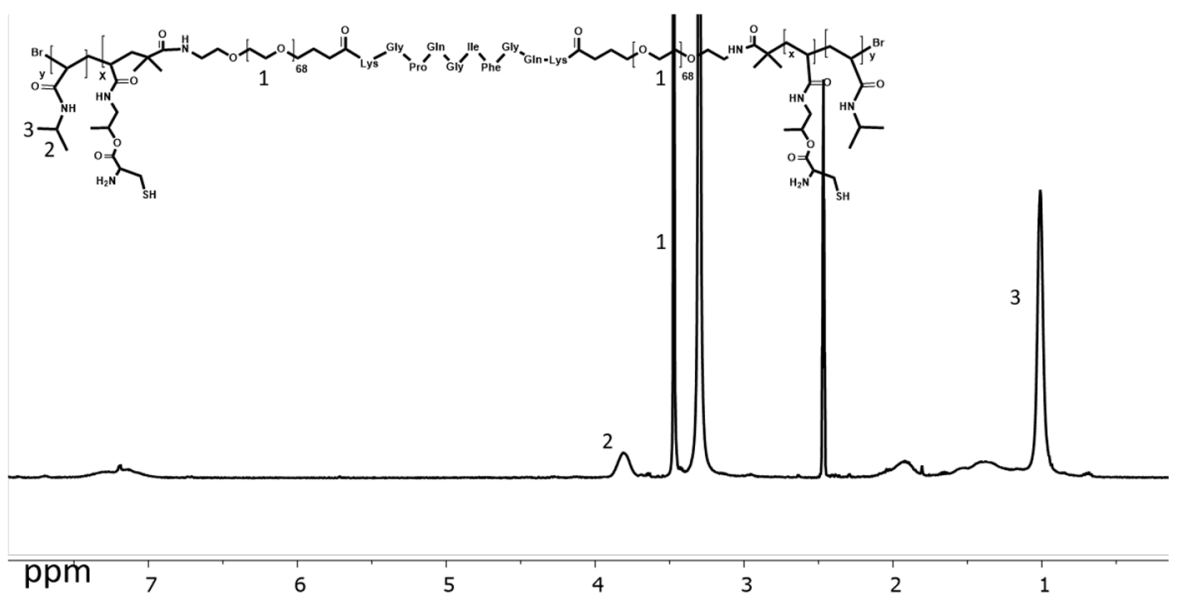

5

4

2

1

SI-Figure 5: A) ${ }^{1} \mathrm{H}-\mathrm{NMR}$ spectrum of protected L-Pep-NC in $\left.\mathrm{CDCl}_{3}, \mathrm{~B}\right){ }^{1} \mathrm{H}-\mathrm{NMR}$ spectrum of L-Pep-NC in deuterated DMSO. 
A

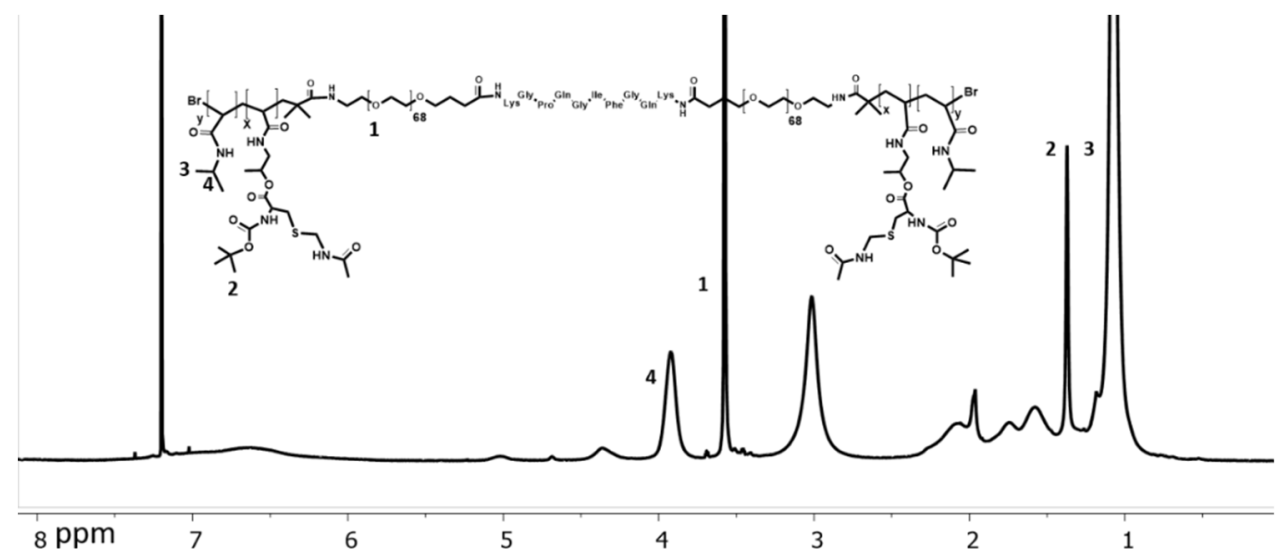

B

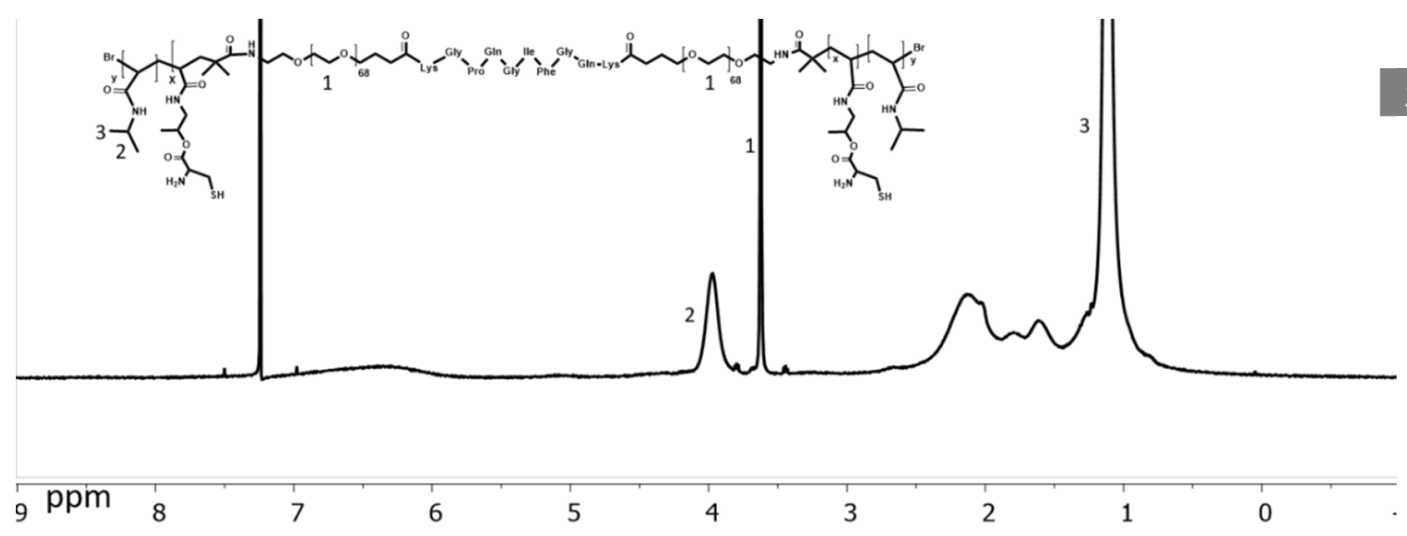

SI-Figure 6: A) ${ }^{1} \mathrm{H}-\mathrm{NMR}$ spectrum of protected D-Pep-NC in $\left.\mathrm{CDCl}_{3} \mathrm{~B}\right){ }^{1} \mathrm{H}-\mathrm{NMR}$ spectrum of D-Pep-NC in $\mathrm{CDCl}_{3}$. 


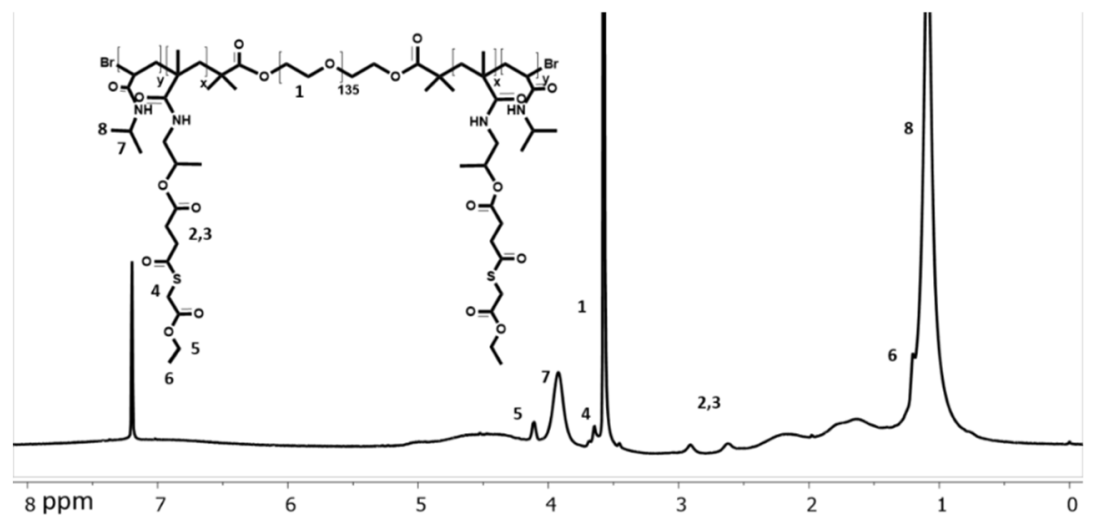

SI-Figure 7: ${ }^{1} \mathrm{H}-\mathrm{NMR}$ spectrum of PNE in $\mathrm{CDCl}_{3}$.

A

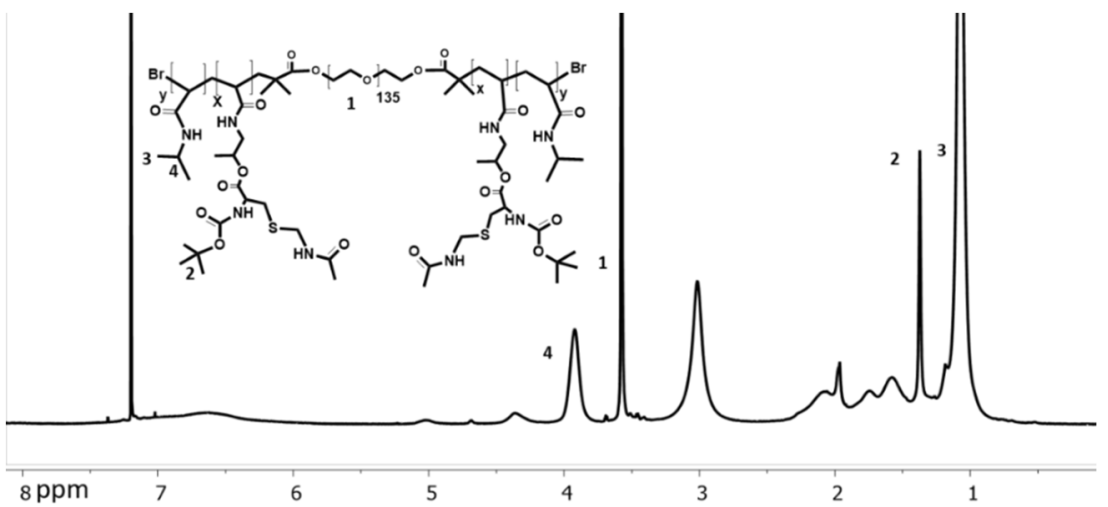

B

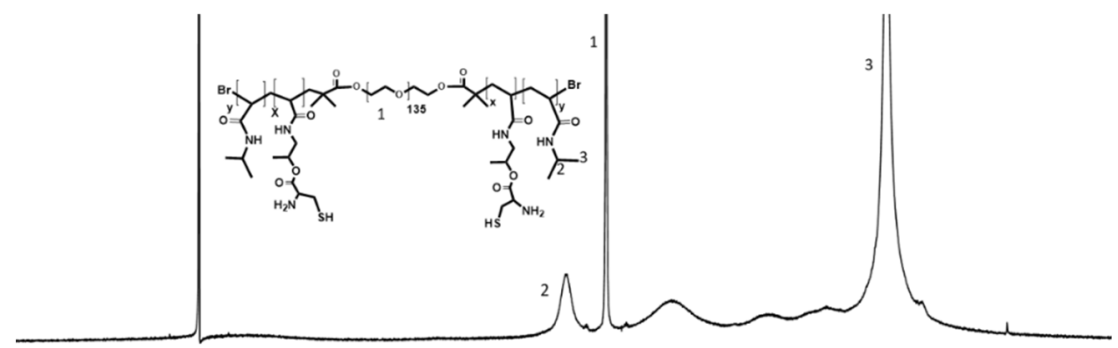

$\begin{array}{llllllllll}\text { ppm } & 8 & 7 & 6 & 5 & 4 & 3 & 2 & 1 & 0\end{array}$

SI-Figure 8: A) ${ }^{1} \mathrm{H}-\mathrm{NMR}$ spectrum of protected $\mathrm{PNC}$ in $\left.\mathrm{CDCl}_{3}, \mathrm{~B}\right){ }^{1} \mathrm{H}-\mathrm{NMR}$ spectrum of $\mathrm{PNC}$ in $\mathrm{CDCl}_{3}$. 


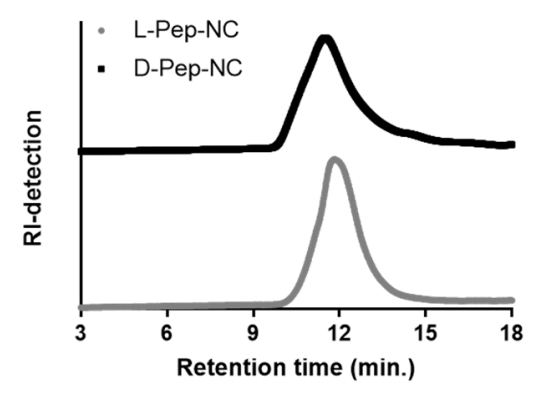

SI-Figure 9: GPC chromatogrammes of L-Pep-NC and D-Pep-NC in DMF containing $10 \mathrm{mM} \mathrm{LiCl.}$

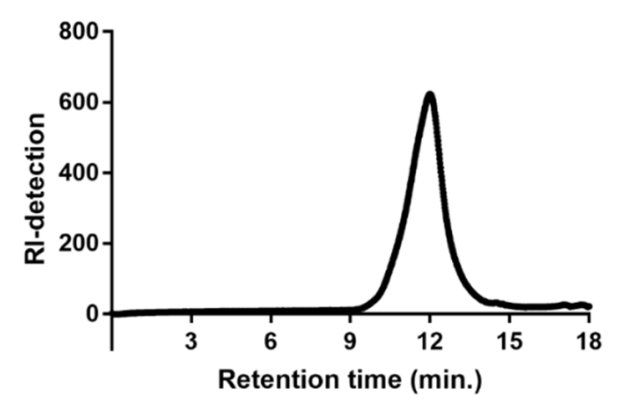

SI-Figure 10: GPC chromatogram of PNE in DMF containing $10 \mathrm{mM} \mathrm{LiCl.}$

A

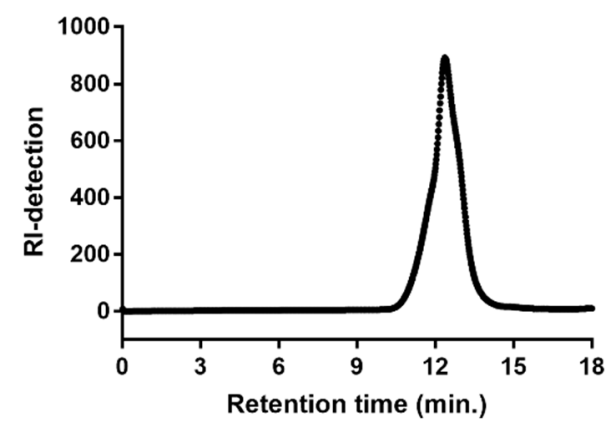

$\mathbf{B}$

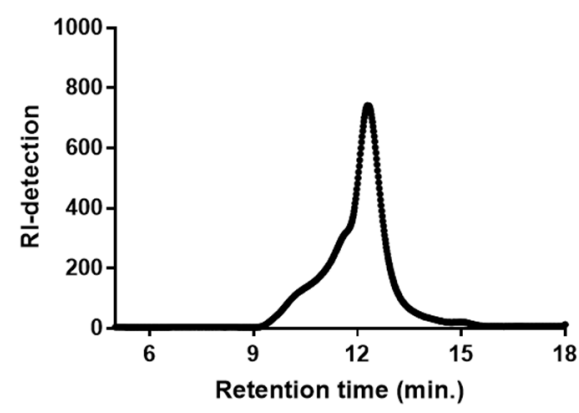

SI-Figure 11: GPC chromatograms of A) protected PNC B) PNC in DMF containing $10 \mathrm{mM} \mathrm{LiCl}$. 


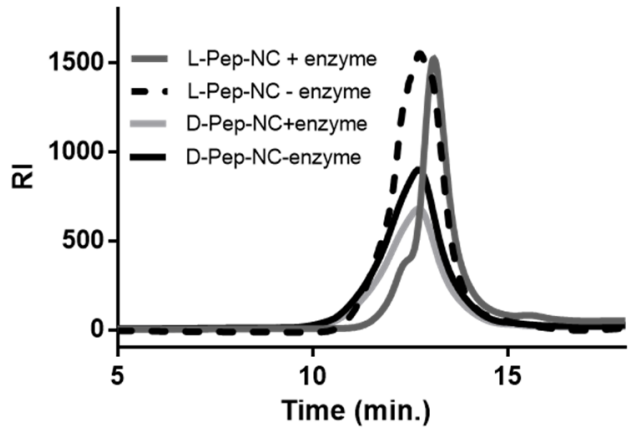

\begin{tabular}{ccc}
\hline \multirow{2}{*}{ Polymer } & \multicolumn{2}{c}{$\mathbf{M}_{\mathbf{n}}(\mathbf{k D a})$} \\
\cline { 2 - 3 } & - enzyme & + enzyme \\
\hline L-pep-NC & 62.3 & 32.8 \\
D-pep-NC & 63.7 & 64.0 \\
\hline
\end{tabular}

SI-Figure 12: GPC chromatograms of L-Pep-NC and D-Pep-NC before and after incubation with collagenase at a concentration of 30 units. $\mathrm{mL}^{-1}$ for 24 hours at $37^{\circ} \mathrm{C}$.

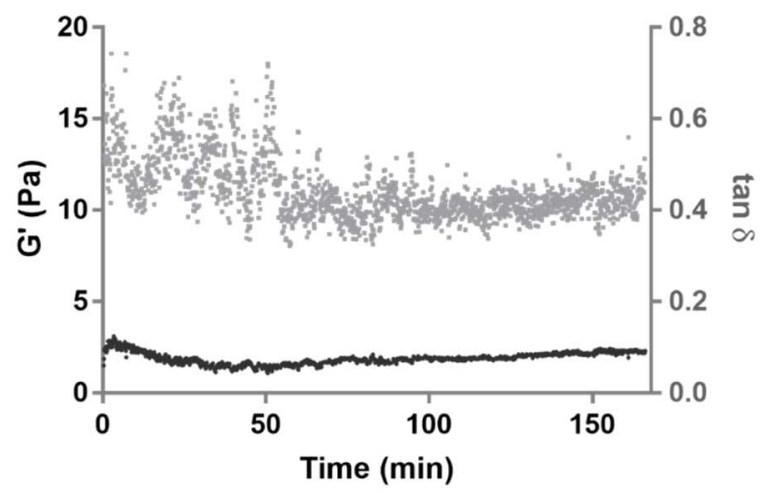

SI-figure 13: Storage modulus $\left(\mathrm{G}^{\prime}\right)$ and $\tan \delta$ as a function of time for micelles at $37^{\circ} \mathrm{C}$. 


\section{Chapter 6}

\section{Rheological properties of dense dispersions of thermosensitive core- crosslinked polymeric micelles}

Marzieh Najafi ${ }^{1}$, Mehdi Habibi², Remco Fokkink ${ }^{3}$, Wim E. Hennink ${ }^{1}$, Tina Vermonden ${ }^{1}$

1 Department of Pharmaceutics, Utrecht Institute for Pharmaceutical Sciences (UIPS), Science for Life, Faculty of Science, Utrecht University, P.O. Box 80082, 3508 TB Utrecht, the Netherlands.

2 Physics and Physical Chemistry of Foods, Wageningen University \& Research, 6708 WG Wageningen Wageningen, the Netherlands

3 Physical Chemistry and Soft Matter, Wageningen University \& Research, Stippeneng 4, 6708 WE Wageningen, the Netherlands 


\section{Abstract}

In this study, temperature dependent behavior of dense dispersions of core crosslinked flower-like micelles is investigated. To this end, micelles were prepared by mixing aqueous solutions of two ABA block copolymers carrying groups that react with each other. The Bblock in the polymers was poly(ethylene glycol) (PEG) and the A-blocks were composed of thermosensitive copolymers of either NIPAM and $\mathrm{N}$-(2-hydroxypropyl) methacrylamidecysteine (HPMA-Cys) (PNC) (molar ratio 91:9) or NIPAM and $\mathrm{N}$-(2-hydroxypropyl) methacrylamide-ethylthioglycolate succinic acid (HPMA-ETSA) (molar ratio 92:8) (PNE). At a temperature $\left(50{ }^{\circ} \mathrm{C}\right.$ ) above the lower critical solution temperature (LCST) of the thermosensitive blocks, self-assembly of the polymers resulted in the formation of flowerlike micelles with a hydrophilic PEG shell and a hydrophobic core based on PNIPAM. The micellar core was stabilized by native chemical ligation (NCL) between thioester groups in HPMA-ETSA and cysteine groups in HPMA-Cys. Above the LCST of the thermosensitive blocks, micelles displayed a radius of $\sim 35 \mathrm{~nm}$, which increased to $\sim 48 \mathrm{~nm}$ by decreasing the temperature below the LCST due to hydration of the PNIPAM core. Concentrated dispersions of these micelles ( $C \geq 7.5 \mathrm{wt} \%$ ) showed glassy state behavior below a critical temperature $\left(T_{C}: 28^{\circ} \mathrm{C}\right)$ which is close to the LCST of PNC $\left(\sim 34^{\circ} \mathrm{C}\right)$ and PNE $\left(\sim 29^{\circ} \mathrm{C}\right)$. The $G^{\prime}$ of the glassy state was dependent on the concentration of micellar dispersions e.g. $~ 700$, 3000 , and $6000 \mathrm{~Pa}$ at concentrations of $10,12.5$ and $15 \mathrm{wt} \%$, respectively, with a $\tan \delta$ of 0.05. Hydration of PNIPAM in the core of crosslinked micelles at temperatures below the $T_{c}$ and the subsequent increase in the micelle volume results in compression of micelles together above a certain concentration and formation of a glass. The effective volume fraction, $\varphi=V_{m} / V_{t}$ in which $V_{m}$ is the micelles' volume at a specific concentration and $V_{t}$ is the sample volume, was used to quantify and compare micelle packing at different concentrations and temperatures. The storage moduli $\left(G^{\prime}\right)$ of the dispersions showed a universal dependence on the effective volume fraction, which increased substantially above a certain effective volume fraction of $\varphi=1.2$. Furthermore, a disordered lattice model describing this behavior fitted the experimental data and revealed a critical volume fraction of $\varphi_{c}=1.35$ close to the experimental value of $\varphi=1.2$. It was shown that two dispersions with equal $\varphi \approx 2.2$ at different temperatures $\left(10\right.$ and $\left.40^{\circ} \mathrm{C}\right)$, different micelles' radius ( $\sim 35$ and $\sim 48 \mathrm{~nm}$ ), and different concentrations (12.5 and $30 \mathrm{wt} \%$ ) displayed a similar $G^{\prime}(\sim 2500 \mathrm{~Pa})$ and yield stress ( $\left.220 \mathrm{~Pa}\right)$ due to equal effective volume fraction validating the proposed model. In contrast to previously studied PNIPAM microgel dispersions, neither precipitation nor gelation of the micelles above the LCST was observed due to the presence of PEG on the micelles' corona. The findings reported in this study provide insights for the 
molecular design of novel thermosensitive PNIPAM nanoparticles with tunable structural and mechanical properties. 


\section{Introduction}

Poly(N-isopropylacrylamide) (PNIPAM) is an attractive polymer for biomedical, biotechnological and pharmaceutical applications due to its thermo-responsive phase transition at a temperature close to body temperature ${ }^{1-4}$. This polymer exhibits a lower critical solution temperature (LCST) in aqueous solution at $32{ }^{\circ} \mathrm{C}$. Below this temperature, the polymeric chains are hydrated and therefore soluble in water, while upon increasing the temperature above the LCST, dehydration occurs and thus the polymer becomes insoluble ${ }^{2}$. Amphiphilic di- or triblock (co)polymers of PNIPAM and polyethylene glycol (PEG) have been extensively studied for the preparation of thermosensitive polymeric micelles as potential drug delivery vehicles ${ }^{5-8}$. During the past years, there is a growing interest in strategies to convert PNIPAM based hydrogels into micelles for drug delivery applications $^{9-11}$.

Unlike micelles, several studies have been devoted to the rheological characterization of dense dispersions of PNIPAM based microgels ${ }^{12-13}$. PNIPAM (sub)micron gel particles are usually synthesized using free radical precipitation polymerization to yield small hydrogel particles ( 0.2 to $0.5 \mu \mathrm{m}$ radius). PNIPAM based microgels display thermosensitivity ${ }^{14}$ and a volume phase transition temperature (VPTT) ${ }^{15}$, which is close to the LCST of the polymer ${ }^{16}$. This reversible transition temperature plays a crucial role in the viscoelastic behavior of dense dispersions of PNIPAM microgels. In general, when the temperature is below the VPTT, soft PNIPAM microgels are swollen resulting in high repulsive interparticle interactions caused by the steric hindrance of swollen polymer chains. At this temperature, the hydrated particles can be packed well above the random close packing fraction $\Phi_{r c p} \approx$ $0.64^{17,18}$ due to compression and deformation of the particles. Under this condition, a glass is formed which exhibits viscoelastic properties resembling pastes and colloidal glasses of hard spheres ${ }^{19-21}$. At temperatures above the VPTT, dehydration of PNIPAM chains leads to shrinkage of the microgels and in this state, a raise in hydrophobic and van der Waals interactions due to high polymer density results in increasing attractive interparticle interactions ${ }^{13}$. A variety of states depending on the magnitude of repulsive and attractive interactions have been reported for PNIPAM microgel particles. A balance between attractive and repulsive interactions results in the formation of colloidal gels ${ }^{22}$, while excessive repulsive interactions lead to a stable liquid dispersion of the microgels ${ }^{23}$, whereas when the attractive forces exceed the repulsive interactions, macroscopic precipitation occurs ${ }^{24}$. Dominancy and magnitude of repulsive or attractive interparticle interactions can be altered by introducing hydrophobic and/or hydrophilic monomers into PNIPAM microgels ${ }^{25}$. For instance, gelation properties of PNIPAM microgels have been tuned by 
changing surface charge density through copolymerization of NIPAM with monomers such as acrylic acid 22,26 , and fumaric acid ${ }^{23}$. At a $p H<p K a$, microgels composed of copolymers of NIPAM and fumaric acid behaved similarly as PNIPAM microgels without the comonomer and displayed colloidal gel behavior at $T>V P T T$. On the other hand at a $p H>p K a$, the surface of the particles is negatively charged due to deprotonation of carboxylic groups, which in turn led to significant charge repulsion between the microgel particles and consequently, due to the high repulsive forces at this $\mathrm{pH}$, the microgels displayed liquid behavior at $T>V P T T^{23}$. Very recently, Es Sayed et al. reported on the synthesis of NIPAM based microgel particles by surfactant-free precipitation copolymerization of NIPAM and PEG-methacrylate. In the so-obtained microgel particles, PEG chains are present on their surface, however, this arrangement is not in a well-defined core-shell structure. They demonstrated that this microgel system has a higher colloidal stability than non-PEGylated ones $^{27}$. However, the effect of PEG on rheological characteristics of microgels was not described in this publication ${ }^{13,27}$. In contrast to microgels, PNIPAM based micelles are nanosized particles (10 -100 nm diameter) formed by self-assembly of amphiphilic block copolymers of PNIPAM-PEG in aqueous solutions at concentrations above the critical micelle concentration (CMC) and above the LCST of PNIPAM block. Therefore, PNIPAM based micelles with a PEG shell have a great potential as a well-defined core-shell structure particle for investigating the effect of PEG on rheological properties of dense PNIPAM based colloids.

In the present contribution, the rheological characteristics of concentrated dispersions of core crosslinked flower-like micelles having a PEG shell and a thermosensitive PNIPAM core were explored. Diluted dispersions of micelles were studied at temperatures above and below the LCST of the polymers using dynamic light scattering (DLS). Subsequently, the rheological properties of a series of concentrated micellar dispersions were studied over a temperature range of 10 to $40{ }^{\circ} \mathrm{C}$ and a theoretical mode ${ }^{28-29}$ was used to describe the observed rheological behavior of the dispersions.

\section{Materials and Methods}

\subsection{Materials}

All commercial chemicals were obtained from Sigma-Aldrich (Zwijndrecht, the Netherlands) and used as received unless indicated otherwise. $N$-(2-hydroxypropyl) methacrylamide (HPMA) was synthesized by a reaction of methacryloyl chloride with 1-aminopropan-2-ol in dichloromethane according to a literature procedure ${ }^{30} . \mathrm{N}$-(2-hydroxypropyl) methacrylamide-Boc-Sacetamidomethyl-L-cysteine (HPMA-Boc-Cys-(Acm)) and N-(2-hydroxypropyl) 
methacrylamide-ethylthioglycolate succinic acid (HPMA-ETSA) were synthesized according to previously reported procedures ${ }^{31,5}$. Viscous Blue 420 Maleimide $^{\mathrm{TM}}$ was obtained from Ursa BioScience (Maryland, USA). Phosphate buffered saline 10X (PBS) pH 7.4 (1.37 M NaCl, $0.027 \mathrm{M} \mathrm{KCl}$ and $0.119 \mathrm{M}$ phosphates) BioReagents was purchased from B. Braun (Melsungen, Germany). Poly(ethylene glycol) bis(2-bromoisobutyrate) with a molecular weight of $6 \mathrm{kDa}$ was synthesized by a method previously reported ${ }^{5}$. Dialysis tubes (molecular weight cutoff (MWCO) $10 \mathrm{kDa}$ ) were obtained from Fisher Scientific (Bleiswijk, the Netherlands). PD-10 desalting columns were purchased from GE Healthcare (Uppsala, Sweden). PEG standards (molecular weights ranging from 106 to $969000 \mathrm{Da}$ ) for GPC characterization were purchased from Agilent Technologies BV (Santa Clara, US).

\subsection{Polymer Synthesis and Characterization}

\subsubsection{Synthesis of P(NIPAM-co-HPMA-Cys)-PEG-P(NIPAM-co-HPMA-Cys), PNC and P(NIPAM-co-HPMA-ETSA)-PEG-P(NIPAM-co-HPMA-ETSA), PNE}

PNC and PNE were synthesized by atom transfer radical polymerization (ATRP) in solvent mixtures of $2.8 \mathrm{~mL}$ water and $0.9 \mathrm{~mL}$ of acetonitrile for PNC and $2.5 \mathrm{~mL}$ water and $2.0 \mathrm{~mL}$ ethanol for PNE. Poly(ethylene glycol) bis(2-bromoisobutyrate $)^{5}(50 \mathrm{mg}, 7.9 \mu \mathrm{mol}), \mathrm{CuBr}(4.5$ $\mathrm{mg}, 31 \mu \mathrm{mol}), \mathrm{CuBr}_{2}(4.7 \mathrm{mg}, 21 \mu \mathrm{mol}), \operatorname{NIPAM}(264 \mathrm{mg} ; 2.3 \mathrm{mmol}$ ) and either HPMA-BocCys-(Acm) (67 mg, $0.16 \mathrm{mmol}$ ) for PNC or HPMA-ETSA (56 mg, $0.16 \mathrm{mmol}$ ) for PNE were dissolved in the corresponding polymerization solvents. The mixtures were deoxygenated by flushing with nitrogen for 15 minutes at room temperature and 15 minutes in an ice bath. Subsequently, $16 \mu \mathrm{L}(60 \mu \mathrm{mol})$ of tris[2-(dimethylamino) ethyl]amine (Me6TREN) as copper ligand was added to the polymerization flasks. The reaction mixtures were stirred for 2 and 5 hours in the ice bath for the synthesis of PNC and PNE, respectively. The crude products were purified by dilution of the reaction mixtures in water to $15 \mathrm{~mL}$, followed by dialysis (MWCO, $10 \mathrm{kDa}$ ) against water at room temperature for two days and subsequently lyophilized. The obtained polymers were characterized using ${ }^{1} \mathrm{H}-\mathrm{NMR}$ (SI-Fig. 1A, 3) and GPC (SI-Fig. 3) analysis. Acetamidomethyl (Acm) and tert-butyloxycarbonyl (Boc) protecting groups of cysteines (in PNC polymer) were removed as described before ${ }^{32}$. Briefly, Boc protecting groups of cysteines were removed by dissolving $250 \mathrm{mg}$ of the protected PNC in dry DCM and trifluoroacetic acid (TFA) (1:1 v/v, $10 \mathrm{~mL})$ and stirred for 1 hour at room temperature. Next, the solvent was evaporated under reduced pressure and the crude product was dissolved in $2 \mathrm{~mL} \mathrm{DCM}$ followed by precipitation in $60 \mathrm{~mL}$ of cold diethyl ether. The Acm protecting group of cysteines was removed by dissolving $250 \mathrm{mg}$ of the Boc deprotected polymer in a mixture of methanol $(\mathrm{MeOH})$ and water $(1: 1 \mathrm{v} / \mathrm{v}, 10 \mathrm{~mL})$ under a 
nitrogen atmosphere. Next, $500 \mu \mathrm{L} \mathrm{HCl}(1 \mathrm{M})$ and $1 \mathrm{~mL}$ iodine $0.2 \mathrm{M}$ in $\mathrm{MeOH}$ were added and left to react while stirring for $1 \mathrm{~h}$ at room temperature. Subsequently, $1 \mathrm{~mL}$ of ascorbic acid $(1 \mathrm{M})$ in water was added to the reaction flask to quench the excess of iodine followed by addition of excess of tris(2-carboxyethyl)phosphine (TCEP) $(250 \mathrm{mg}$ ) to reduce the formed disulfide bonds and the reaction mixture was subsequently stirred overnight. Finally, the mixture was dialyzed against water for 2 days at room temperature (MWCO, 10 $\mathrm{kDa})$ and the final product was obtained after lyophilization and characterized by ${ }^{1} \mathrm{H}-\mathrm{NMR}$ (SI-Fig. 1B) and GPC (SI-Fig. 3).

\subsubsection{NMR Spectroscopy Analysis}

The obtained polymers were characterized by ${ }^{1} \mathrm{H}-\mathrm{NMR}$ using a Bruker 600 UltraShield ${ }^{\mathrm{TM}}$ spectrometer (Billerica, Massachusetts, USA). The chemical shifts were calibrated against the residual solvent peak of $\mathrm{CHCl}_{3}(\delta=7.26 \mathrm{ppm})$.

\subsubsection{Gel Permeation Chromatography (GPC) Analysis}

The molecular weights and molecular weight distribution of the synthesized polymers were determined using a Waters 2685 Alliance 2414 System (Waters Corporation, Milford, MA) equipped with a refractive index detector. Samples were dissolved in DMF containing 10 $\mathrm{mM} \mathrm{LiCl}$ at a concentration of $5 \mathrm{mg} \cdot \mathrm{mL}^{-1}$ and separation was carried out by 2 PLgel $5 \mu \mathrm{m}$ Mixed-D columns (Polymer Laboratories, UK) in series at a temperature of $65^{\circ} \mathrm{C}$ using the same solvent as mobile phase. The flow rate was $1 \mathrm{~mL}$. $\mathrm{min}^{-1}$ and a series of linear PEGs with narrow and defined molecular weights were used as calibration standards ${ }^{33}$. Data were recorded and analyzed with Empower software v.3. 2010.

\subsubsection{Determination of Cloud Point of PNC and PNE}

The could point (CP) of the synthesized polymers was determined using a Jasko FP-8300 spectrofluorometer (JASCO, Tokyo, Japan). The polymers were dissolved at a concentration of $1 \mathrm{mg} \cdot \mathrm{mL}^{-1}$ in PBS (0.13 M NaCl, $2.7 \mathrm{mM} \mathrm{KCl}$ and $11.9 \mathrm{mM}$ phosphates, $\mathrm{pH}$ 7.4). The temperature was ramped from 10 to $50{ }^{\circ} \mathrm{C}$ at $1{ }^{\circ} \mathrm{C} \cdot \mathrm{min}^{-1}$ and the measurements were performed at $650 \mathrm{~nm}$. The $\mathrm{CP}$ was taken as the onset of increasing scattering intensity.

\subsection{Micelle Preparation and Characterization}

\subsubsection{Preparation of the Core Crosslinked Flower-like Micelles}


To form micelles, PNC and PNE were dissolved separately in PBS $(0.13 \mathrm{M} \mathrm{NaCl}, 2.7 \mathrm{mM} \mathrm{KCl}$ and $11.9 \mathrm{mM}$ phosphates, $\mathrm{pH} 7.4$ ) at a concentration of $10 \mathrm{mg} \cdot \mathrm{mL}^{-1}$ and left at $4{ }^{\circ} \mathrm{C}$ for 3 hours. Subsequently, the polymer solutions were mixed in a 1:1 volume ratio (i.e. 1:1 molar ratio of HPMA-Cys to HPMA-ETSA) and heated to $50^{\circ} \mathrm{C}$ using an oil bath. The mixture was stirred at $50{ }^{\circ} \mathrm{C}$ overnight. The resulting micellar dispersion was dialyzed against water (MWCO, $10 \mathrm{kDa}$ ) for two days at room temperature and subsequently lyophilized.

\subsubsection{Labeling Micelles with a Viscosity Fluorescence Probe}

Micelles were prepared as described in section 2.3.1. Subsequently, the buffer medium was refreshed, and the micelles were purified by PD-10 desalting columns equilibrated with PBS (0.13 M NaCl, $2.7 \mathrm{mM} \mathrm{KCl}$ and $11.9 \mathrm{mM}$ phosphates, $\mathrm{pH} 7.4$ ) according to the protocol provided by the supplier. Next, $10 \mu \mathrm{L}$ of a $50 \mathrm{mg} \cdot \mathrm{mL}^{-1}$ Viscous Blue 420 Maleimide ${ }^{\mathrm{TM}}$ stock solution in DMSO was added to $1 \mathrm{~mL}$ micellar dispersion and left to react at $4{ }^{\circ} \mathrm{C}$ for two days under a nitrogen atmosphere ${ }^{34-35}$. Next, the labeled micelles were purified three times by PD-10 desalting columns equilibrated with PBS to remove non-conjugated dye.

\subsubsection{Dynamic Light Scattering}

The radius of micelles at different temperatures was measured at a concentration of 3 mg. $\mathrm{mL}^{-1}$ in PBS (0.13 M NaCl, $2.7 \mathrm{mM} \mathrm{KCl}$, and $11.9 \mathrm{mM}$ phosphates, $\mathrm{pH} 7.4$ ) using an ALV7004 correlator, ALV/LSE-5004 Goniometer, ALV/Dual High QE APD detector unit with fiber splitting device with a set-up of 2 off detection system (Langen, Germany). A Uniphase Model 1145P He-Ne Laser was used at a laser wavelength of $632.8 \mathrm{~nm}$ and power of $22 \mathrm{~mW}$. The measurements were performed at a $90^{\circ}$ angle. The temperature was increased from 10 to $40{ }^{\circ} \mathrm{C}$ with 10 minutes equilibration time at each temperature. The temperature was controlled by a Julabo CF41 (Seelbach, Germany) thermostatic bath and changes in viscosity were taken into account by the software.

\subsubsection{Zeta Potential}

The prepared micelles in PBS were diluted (1:20 v/v) in HEPES $(20 \mathrm{mM}, \mathrm{pH} 7.0)$ to a final concentration of $0.5 \mathrm{mg} \cdot \mathrm{mL}^{-1}$ and the zeta potential was measured at 10 and $40{ }^{\circ} \mathrm{C}$ using a Zetasizer Nano Z (Malvern Instruments Ltd, Malvern, UK).

\subsubsection{Fluorescence Intensity Measurements}

The fluorescence intensity of dye-conjugated micelles was measured using a Jasko FP-8300 (JASKO, Tokyo, Japan) spectrofluorometer; the excitation and emission wavelengths were 
set at 320 and $400 \mathrm{~nm}$, respectively. Micelles were dispersed at a concentration of $3 \mathrm{mg} \cdot \mathrm{mL}^{-}$ ${ }^{1}$ in PBS (0.13 M NaCl, $2.7 \mathrm{mM} \mathrm{KCl}$ and $11.9 \mathrm{mM}$ phosphates, $\mathrm{pH} \mathrm{7.4)} \mathrm{and} \mathrm{heated} \mathrm{from} 10$ to $40{ }^{\circ} \mathrm{C}$. Samples were allowed to equilibrate at each temperature for 10 minutes before measurement.

\subsection{Micelle Dispersion Preparation and Characterization}

\subsubsection{Preparation of Dense Micellar Dispersions}

Lyophilized micelles were dispersed in PBS $(0.13 \mathrm{M} \mathrm{NaCl}, 2.7 \mathrm{mM} \mathrm{KCl}$ and $11.9 \mathrm{mM}$ phosphates, $\mathrm{pH} 7.4$ ) at concentrations of 7.5, 10, 12.5, 15, 20, and $30 \mathrm{wt} \%$ and left to hydrate for 3 hours at $4{ }^{\circ} \mathrm{C}$ prior to the measurement. For instance, a sample at a concentration of $20 \mathrm{wt} \%$ was prepared by dispersing $200 \mathrm{mg}$ of lyophilized micelles in 800 $\mu \mathrm{L}$ of PBS. Details of the preparation of individual samples are provided in supporting information section 2, SI-table 2.

\subsubsection{Rheological Characterization of Dense Micellar Dispersions}

Rheological analyses of dense micellar dispersions were performed on a DHR-2 rheometer (TA Instruments, New Castle, DE) using a $20 \mathrm{~mm}$ aluminum cone $\left(1^{\circ}\right)$ geometry equipped with a solvent trap. Temperature sweeps were performed from 40 to $10{ }^{\circ} \mathrm{C}$ at $1{ }^{\circ} \mathrm{C}$ per minute steps with a 30 second equilibration time for each temperature. The measurements were conducted at a fixed frequency of $1 \mathrm{~Hz}$ and a strain of $1 \%$ and the sample volume was $70 \mu \mathrm{L}$.

The yield stress measurements were performed using 12.5 and $30 \mathrm{wt} \%$ dispersions, at temperatures of 10 and $40{ }^{\circ} \mathrm{C}$, respectively. The samples $(70 \mu \mathrm{L})$ were subjected to a strain sweep from 1 to $40 \%$ at oscillation frequency of $1 \mathrm{~Hz}$.

\section{Results and Discussions}

\subsection{Polymer Synthesis and Characterization}

Figure 1 shows the structures of polymers investigated in this study synthesized using atom transfer radical polymerization (ATRP) ${ }^{36}$. As reported previously ${ }^{5}$, PNE and protected PNC were obtained by copolymerization of NIPAM/HPMA-ETSA and NIPAM/HPMA-Boc- Cys$(\mathrm{Acm})$, respectively. The feed molar ratio of the monomers in both polymerizations was 93:7 and a poly(ethylene glycol) bis(2-bromoisobutyrate) with a number-average molecular weight $\left(\mathrm{Mn}_{\mathrm{n}}\right)$ of $6 \mathrm{kDa}$ was used as bifunctional ATRP initiator ${ }^{5}$. The protected PNC triblock 
copolymer was obtained with a yield of $94 \%$ and the $M_{n}$ of the polymer was $\sim 42 \mathrm{kDa}$ as determined by ${ }^{1} \mathrm{H}-\mathrm{NMR}$ (SI. Fig. 1A) and $\sim 69 \mathrm{kDa}$ (PDI 1.5) by GPC analysis (table 1, SI. Fig. 3). The purified PNE was obtained with a yield of $85 \%$ and had an $M_{n}$ of $\sim 41 \mathrm{kDa}$ as determined by ${ }^{1} \mathrm{H}-\mathrm{NMR}$ analysis (SI. Fig. 2) and $\mathrm{Mn}_{\mathrm{n}}$ of $\sim 64 \mathrm{kDa}$ (PDI of 1.8) as determined by GPC (Table 1, SI. Fig. 3). The difference in the recorded Mn's by GPC and NMR analysis of these polymers can be ascribed to the presence of strong inter- and intramolecular hydrogen bonding in PNIPAM based polymers as reported before ${ }^{37,38}$.

The incorporation of HPMA-Cys and HPMA-ETSA units in the thermosensitive domains of the polymers provides terminal cysteine and thioester functionalities that can be exploited for native chemical ligation (NCL) ${ }^{39}$. PNC underwent a deprotection procedure $\mathrm{e}^{31}$ to remove $\mathrm{Acm}$ and Boc protecting groups to yield a polymer with free cysteine moieties. ${ }^{1} \mathrm{H}-\mathrm{NMR}$ analysis showed that the deprotection of cysteines indeed was successful (SI-Fig. 1B) and GPC analysis showed an $\mathrm{Mn}_{\mathrm{n}}$ of $\sim 72 \mathrm{kDa}$ for the deprotected polymer. As expected, both polymers exhibited a cloud point of $34.1^{\circ} \mathrm{C}$ for PNC and $29.2^{\circ} \mathrm{C}$ for PNE due to the presence of thermosensitive PNIPAM-blocks and to the obtained values were similar as reported before for the same polymers ${ }^{5}$ (Table 1 ).

Table 1: Characteristics of the synthesized ABA triblock copolymers of (protected) PNC and PNE. Both ABA polymers contain PEG (6 $\mathrm{kDa}$ ) as midblock, whereas the thermosensitive outer blocks are composed of either NIPAM and HPMA-ETSA (PNE) or NIPAM and HPMA-Boc-Cys-(Acm) (protected PNC). The feed molar ratios of NIPAM to HPMA-Boc-Cys-(Acm) and HPMA-ETSA were 93:7.

\begin{tabular}{|c|c|c|c|c|c|c|c|}
\hline \multirow{2}{*}{ Polymer } & \multicolumn{2}{|c|}{ Obtained molar ratio a } & \multirow{2}{*}{$\begin{array}{l}\mathrm{M}_{\mathrm{n}}^{\mathrm{a}} \\
(\mathrm{kDa})\end{array}$} & \multirow{2}{*}{$\begin{array}{l}\mathrm{M}_{\mathrm{n}}^{\mathrm{b}} \\
(\mathrm{kDa})\end{array}$} & \multirow{2}{*}{$P D I^{b}$} & \multirow{2}{*}{$\begin{array}{l}\mathrm{CP} \\
\left({ }^{\circ} \mathrm{C}\right)\end{array}$} & \multirow{2}{*}{$\begin{array}{c}\text { Yield } \\
\text { (\%) }\end{array}$} \\
\hline & [NIPAM]:[HPMA-Boc-Cys-Acm] & [NIPAM]:[HPMA-ETSA] & & & & & \\
\hline PNC & $91: 9$ & - & 42.1 & 69.1 & 1.5 & $34.1^{\mathrm{C}}$ & 94 \\
\hline PNE & - & $92: 8$ & 40.9 & 63.9 & 1.8 & 29.2 & 85 \\
\hline
\end{tabular}

a Determined by ${ }^{1} \mathrm{H}-\mathrm{NMR}$. ${ }^{\mathrm{b}}$ Determined by GPC. ${ }^{\mathrm{c}}$ Cloud point of the deprotected polymer 


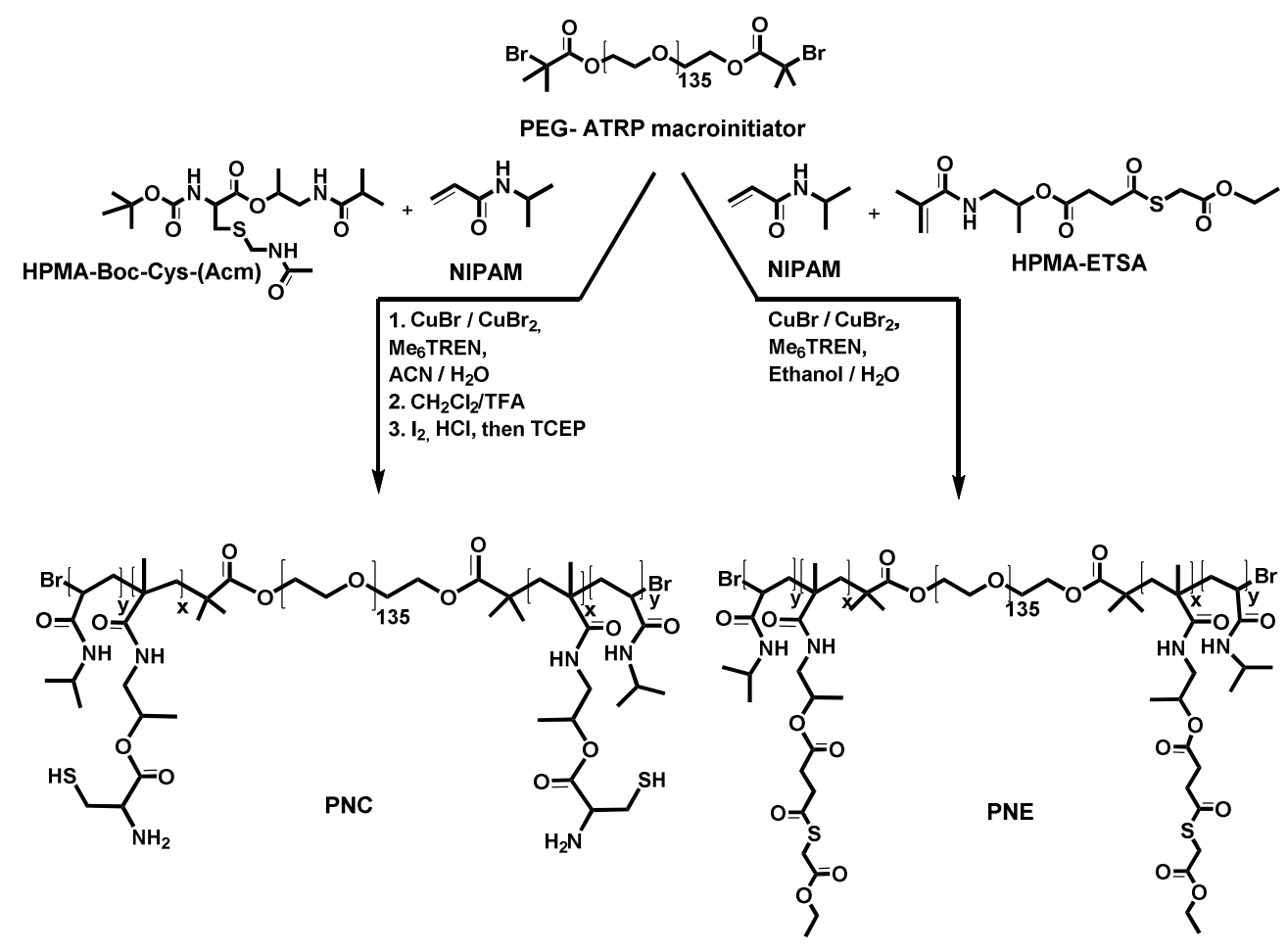

Figure 1: Reaction schemes for the synthesis of PNC and PNE.

\subsection{Micelle Formation and Characterization}

Micelles were prepared following a previously reported method by mixing PNC and PNE solutions (10 mg. $\mathrm{mL}^{-1}$ ) at a volume ratio of 1:1 (i.e. molar ratio 1:1 for HPMA-Cys and HPMAETSA) followed by increasing the temperature to $50{ }^{\circ} \mathrm{C}^{5}$, 40 . The close proximity of thioester and thiol functionalities in the micellar core induces native chemical ligation leading to the stabilization of the micellar structures (Fig. 2A). Figure 2B shows the radius of the micelles as a function of temperature in a dilute dispersion ( $3 \mathrm{mg} \cdot \mathrm{mL}^{-1}$ ) as measured by $\mathrm{DLS}$. By varying the temperature from 10 to $40{ }^{\circ} \mathrm{C}$, the radius of the micelles changed from $\sim 48$ to $\sim 35 \mathrm{~nm}$ with an inflection point at approximately $28^{\circ} \mathrm{C}$, which is close to the LCST of both polymers. Static light scattering at 10 and $40{ }^{\circ} \mathrm{C}$ demonstrated that the micelle's molecular weights $\left(\sim 15.5 \times 10^{6} \mathrm{kDa}\right)$ and aggregation number $\left(\mathrm{N}_{a g g}\right)(\sim 400)$ were equal within the experimental error confirming the formation of core crosslinked micelles. SLS data has been reported before for this type of micelles ${ }^{5}$, but for sake of readability are also shown in $\mathrm{SI}$ table 1. The micelles displayed a $\zeta$-potential of $-2.3 \pm 0.4$ and $-4.2 \pm 0.2 \mathrm{mV}$ at 10 and $40{ }^{\circ} \mathrm{C}$, 
respectively, showing a slight negative charge of the micelles above and below the LCST, in line with findings for other pegylated polymeric nanoparticles ${ }^{41}$.

To visualize the shrinking of the micellar core due to dehydration of PNIPAM chains above the LCST, a rigidochromic fluorescent probe was used ${ }^{35}$ 42. Rigidochromic fluorescent probes are excited by absorbing a photon of a specific wavelength. After excitation, there are two possible pathways to deactivate the excited-state: non-radiative and radiative. The non-radiative pathway (non-fluorescent) results in rotation of a specific bond that prevents effective $\pi$ conjugation in the molecule. When this pathway is hampered by confinement induced by molecular structural changes or enhanced viscosity of the environment, the probe is forced to follow the radiative pathway and emit light to the deactivated excited state $^{43}$. To this end, the maleimide functionalized rigidochromic dye (Viscous Blue 420 Maleimide ${ }^{\mathrm{TM}}$ ) was conjugated to free thiol groups in the micellar core remaining after native chemical ligation and/or to non-reacted cysteine moieties in the micellar core. This conjugation positioned the rigidochromic molecule as a confinement probe in the PNIPAM core of the micelles. The fluorescence intensity of dye conjugated micelles was monitored as a function of temperature (Fig. 2C). First, a decrease in the fluorescence intensity with increasing temperature from 10 to $25^{\circ} \mathrm{C}$ was observed, as it has been observed before for this dye in aqueous solution which was attributed to the decrease in viscosity of water with temperature ${ }^{34}$. Since the core in this temperature range $\left(10\right.$ to $\left.25^{\circ} \mathrm{C}\right)$ is highly hydrated, the lower fluorescence intensity with increasing temperature can be explained by the low local viscosity in the micellar core. Interestingly, by further increasing the temperature from 25 to $40{ }^{\circ} \mathrm{C}$, the fluorescence intensity did not further decrease but instead increased. Most likely, the dehydration of PNIPAM and accordingly shrinkage of the micellar core resulted in an increase in the confinement of the rigidochromic probes. Therefore, these molecules follow the radiative pathway which in turn resulted in an increase in fluorescence intensity. Figure 2D shows the change in fluorescence intensity as a function of the radius of the micelles. From 10 to $25^{\circ} \mathrm{C}$, when the radii are hardly changed (from 47 to $45 \mathrm{~nm}$ ), a decrease in fluorescence intensity from 680 to 590 A.U. was observed as explained above. With decreasing the radius from 45 to $35 \mathrm{~nm}$ (from 25 to $35^{\circ} \mathrm{C}$ ) a significant change in intensity from 590 to 850 A.U. was observed, which shows that indeed shrinkage of micelles due to the dehydration of PNIPAM chains increased dye confinement and consequently increased dye intensity. Subsequently, when the radius of the micelles remained relatively constant, (35.7 to $35.0 \mathrm{~nm}$ ) at a temperature from 35 to $40{ }^{\circ} \mathrm{C}$, fluorescence intensity slightly decreased again, which indicates a slight decrease in viscosity of the dye microenvironment. According to Wu et al. PNIPAM based nanoparticles retain water ( 70\%) in their hydrophobic core even in a highly collapsed state at temperatures above the $\mathrm{LCST}^{15}$. 
Therefore, probably a decrease in the viscosity of water in the micellar core upon increasing temperature resulted in an overall decrease in the viscosity of dye microenvironment, which led to a slight decrease in dye intensity.
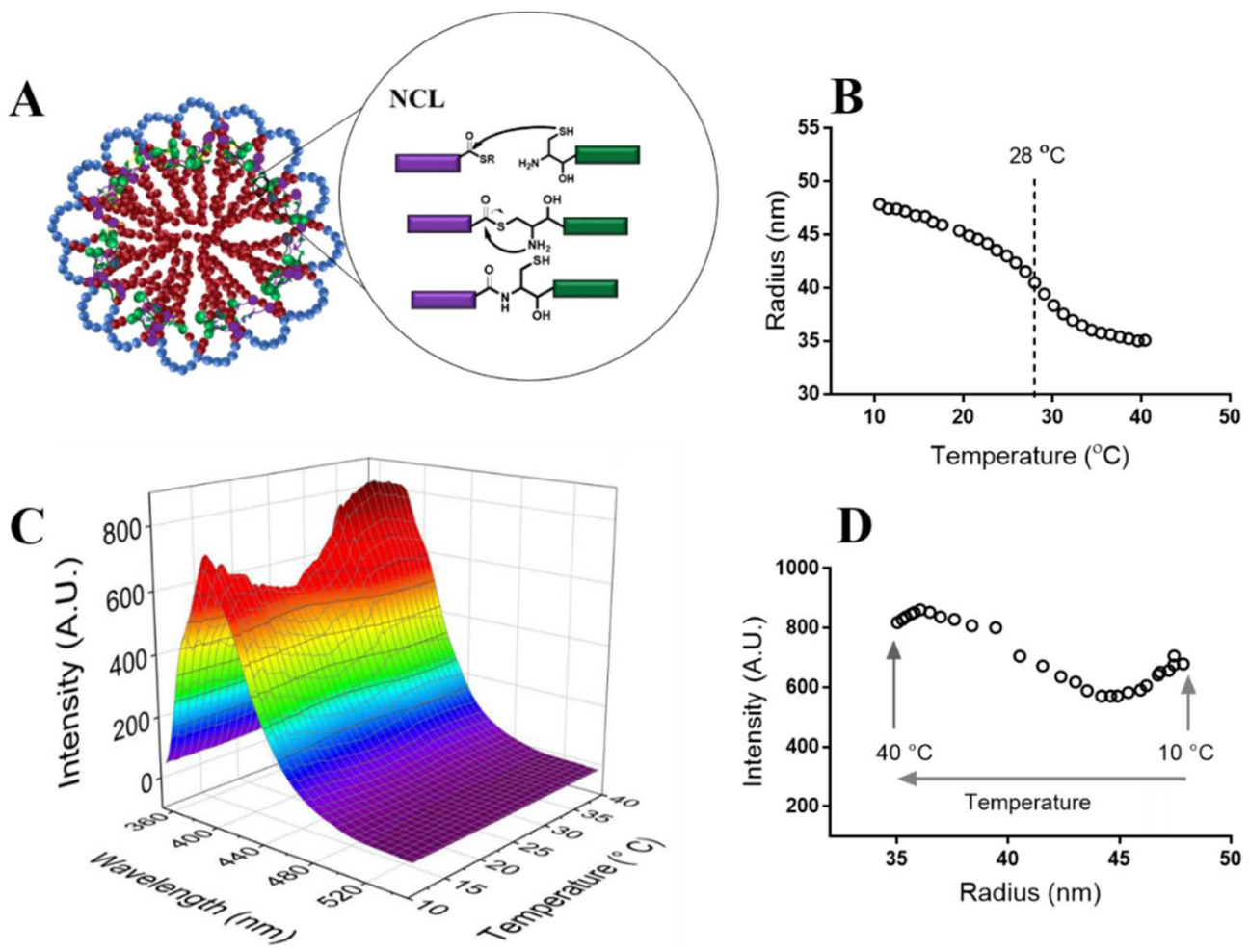

Figure 2: A) Schematic view of the flower-like micelles crosslinked by native chemical ligation ${ }^{5}$. B) The change in the radius of micelles as a function of temperature at a micelle concentration of $3 \mathrm{mg} \cdot \mathrm{mL}^{-1}$. C) Change in the dye intensity as a function of temperature at different wavelengths. D) Change in the dye fluorescence intensity as a function of the micelle radius at the wavelength of $387 \mathrm{~nm}$.

\subsection{Rheological Characterization of Dense Micellar Dispersions}

The rheological properties of dense micellar dispersions were investigated as a function of temperature. The number of micelles $(n)$ in different dispersions was calculated based on the concentration of samples and the molecular weight of the micelles (the details are shown in supporting information section 2, SI-table 2). This number was used to calculate the total volume of micelles $\left(V_{m}\right)$ in each dispersion based on the radius of the micelles in a dilute dispersion ( $3 \mathrm{mg} \cdot \mathrm{mL}^{-1}$ ). In addition, an effective volume fraction (i.e. packing 
fraction), defined as $\varphi=V_{m} / V_{t}$ in which $V_{t}$ is the volume of a micelle dispersion, is reported (see supporting information section 2, SI-table 2). The calculations show that the total volume of micelles $\left(V_{m}\right)$ at concentrations $\geq 7.5 \mathrm{wt} \%$ and at $10{ }^{\circ} \mathrm{C}$ is larger than $V_{t}$ (SI-table 2). This indicates that the effective volume fraction $(\varphi)$ can be even above the maximum random packing fraction for monodisperse hard spheres $\Phi_{r c p} \approx 0.64^{17,18}$ possibly due to compressible deformation of the relatively soft micelles. Consequently, at the same temperature the volume of a given micelle in a dilute dispersion is larger than its volume in a concentrated dispersion as visualized schematically in Fig. 3.

A

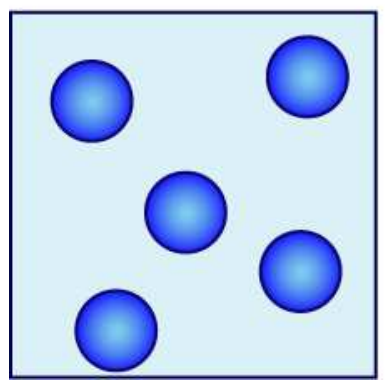

B

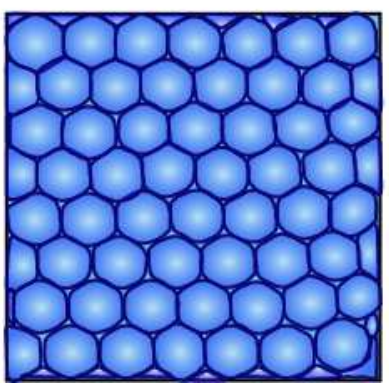

Figure 3: A) Micelles in a dilute dispersion B) Compression of micelles in a concentrated dispersion.

The storage modulus $\left(G^{\prime}\right)$ of several micellar dispersions was measured while the temperature was increased from 10 to $40{ }^{\circ} \mathrm{C}$ (Fig. 4A). The sample at a concentration of 7.5 wt\% displayed a low $G^{\prime}$ of $\sim 10$ Pa with $\tan \delta \approx 0.85$ at $10^{\circ} \mathrm{C}$. By increasing temperature to $40^{\circ} \mathrm{C}$, this sample displayed a liquid state $(\tan \delta \approx 4)$. Below the LCST $\left(10^{\circ} \mathrm{C}\right)$, samples at concentrations of $10,12.5$, and $15 \mathrm{wt} \%$ showed $G^{\prime}$ values of 700,3000 , and $6000 \mathrm{~Pa}$, respectively with $\tan \delta \approx 0.05$, demonstrating solid-like behavior with a concentration dependent $G^{\prime}$. By increasing the temperature above the LCST $\left(40{ }^{\circ} \mathrm{C}\right)$, the $G^{\prime}$ values decreased significantly to about a few Pascals ( $<2 \mathrm{~Pa}$ ) with $\tan \delta \geq 1$, indicating a transition from solid-like to liquid-like. The samples with 20 and 30 wt\% micelles exhibited substantial $G^{\prime}$ values of $\sim 17000$ and $\sim 28000 \mathrm{~Pa}$, respectively, with $\tan \delta \sim 0.05$ below the LCST $\left(10^{\circ} \mathrm{C}\right)$, and above the LCST $\left(40{ }^{\circ} \mathrm{C}\right.$ ), their $G^{\prime}$ decreased to $\sim 150 \mathrm{~Pa}$ ( $\tan \delta \sim 0.5$ ) and $\sim 3000 \mathrm{~Pa}(\tan \delta \sim 0.2)$, respectively.

The temperature dependency of storage modulus for micellar dispersions differing in concentrations can be described by critical-like behaviour: $G^{\prime} \sim\left(T_{C}-T\right)^{\gamma} 44-45$, in which $T_{c}$ is the critical temperature and $\gamma$ is the critical exponent. The critical temperature $T_{C}$ is set at $28{ }^{\circ} \mathrm{C}$ as evidenced from the sharp decrease in the experimental values of $G^{\prime}$ in 
samples with 10, 12.5, and $15 \mathrm{wt} \%$ (Fig. 4A). This temperature coincides with the inflection point of the size-temperature curve in Fig. 2 and is close to the LCST of PNC $\left(\sim 34{ }^{\circ} \mathrm{C}\right)$ and PNE ( $\left.29^{\circ} \mathrm{C}\right)$. The solid curves in Fig. $4 \mathrm{~A}$ show the best fits of the above equation to data points of samples $10,12.5$, and $15 \mathrm{wt} \%$ with a fixed $T_{C}=28^{\circ} \mathrm{C}$ and yielded $\gamma$ values of 2.1 , 1.6 , and 1.3 (all with an absolute error of $\sim 0.1$ ), respectively. This demonstrates that the critical exponent $\gamma$ decreases with increasing concentration of the samples. Below the critical temperature, the core of micelles is hydrated, which results in an increase in micelle size ( $\sim 35 \mathrm{~nm}$ vs. $\sim 48 \mathrm{~nm}$ at 40 and $10^{\circ} \mathrm{C}$, respectively, Fig. $2 \mathrm{~B}$ ). Consequently, micelles were jammed together $(\varphi \geq 1.8)$ and formed a colloidal glass with a substantial value of $G^{\prime}$. Increasing temperature above $T_{C}$ and consequent reduction in the size of micelles resulted in a decrease in the effective volume fraction $(\varphi \leq 1)$, and thus the average number of neighbors in direct contact with each other decreases ${ }^{28}$ resulting in a lower $G^{\prime}$ value and also in flow ( $\tan \delta \geq 1$ ). This observation suggests that $\varphi$ is the main parameter controlling the rheological properties of the system. The samples with concentrations of 20 and $30 \mathrm{wt} \%$ did not show a sharp transition at $28{ }^{\circ} \mathrm{C}$ but represented a solid-like behavior above and below this temperature. This is due to the fact that at these concentrations $\varphi$ values are so high $(1.4 \leq \varphi \leq 5.6)$ that the reduction in the size of micelles is not significant enough to result in a glass-liquid transition ( $\tan \delta \leq 0.5$ below and above $T_{C}$ ). Therefore, the reduction in the size of micelles only reduces the extend of compression of micelles into each other which results in a lower $G^{\prime}$ and a higher $\tan \delta$ above $T_{c}$ (e.g. micellar dispersion $20 \mathrm{wt} \%$ displayed a $G^{\prime}$ of $150(\tan \delta$ of 0.5$)$ and $17000 \mathrm{~Pa}(\tan \delta$ of 0.05 ) at 40 and $10^{\circ} \mathrm{C}$, respectively).

Fig. 4 B shows $G^{\prime}$ as a function of $\varphi$ for micellar dispersions of different concentrations and temperatures. This figure shows that data points of different samples coincide on a master curve demonstrating that $\varphi$ is the main parameter controlling the transition behavior of the system. As shown in this figure, $G^{\prime}$ substantially increases at a $\varphi$ of 1.2 (black arrow) above which the system shows solid-like behavior with $0.05 \leq \tan \delta \leq 0.85$. To quantitatively link the sharp transition of $G^{\prime}$ to $\varphi$, a disordered lattice model for hardsphere colloidal glasses was used that gives the non-affine expression of the storage modulus as $G^{\prime} \sim \varphi\left(z-z_{c}\right)$, in which $z$ is the average number of neighbors of one micelle in the dispersion (coordination number) ${ }^{28,29}$ and $Z_{c}=4$ is the critical number at the glass transition when $\varphi=\varphi_{c}{ }^{28} . \varphi_{c}$ is the critical volume fraction at which the glass transition occurs. According to Zaccone et al. ${ }^{28}, \varphi$ and $z$ are related via $z-z_{c}=\left(\varphi-\varphi_{c}\right)^{b}$. The exponent $b$ depends on the micelle-micelle interaction, including steric contributions. For the micellar system investigated in this study, $b=1$ provides a good description of the experimental data and indicates that $\varphi$ and $z$ are linearly proportional. Therefore, $z-$ 
$z_{c}$ can be replaced by $\varphi-\varphi_{c}$, leading to $G^{\prime}=k \varphi\left(\varphi-\varphi_{c}\right)$. The solid curve in Fig. 4B shows that $G^{\prime}$ as a function of $\varphi$ is well described by this model when $b$ is fixed at 1 , yielding the fitting parameters $k=1575 \mathrm{~Pa}$ and $\varphi_{c}=1.35$. This finding is supported by the data of Fig. $4 \mathrm{~B}$ in which it is shown that the two high concentration micellar dispersions display $1.4 \leq \varphi \leq 5.6$ over the whole temperature range which is well above $\varphi_{c}$. Therefore, these two samples only display solid state behavior above and below $T_{c}$ and do not exhibit a transition from solid- to liquid state.

The behavior of our micellar system is in contrast with previous studies on PNIPAM microgels. For example, Weitz et al. ${ }^{26}$ observed that a dense dispersion of PNIPAM microgel particles (radius of $260 \mathrm{~nm}$ at $41^{\circ} \mathrm{C}$ ) first showed a glass transition and then after liquefication formed a gel upon an increase in temperature above the LCST which is ascribed by the authors to the formation of stable clusters of aggregated PNIPAM microgels above the $\operatorname{LCST}^{26}$. The different behavior of the micelle dispersion system described in this study compared to the previous microgel systems could be attributed to the presence of the PEG corona that covers the micelles. Hence, above the LCST the hydrophilic PEG corona is exposed (SI-Fig. 4), which avoids hydrophobic interactions between the dehydrated and hydrophobic PNIPAM cores and consequent particle aggregation. Besides, the slightly negative zeta potential $(-4.2 \pm 0.2 \mathrm{mV})$ may also contribute to the stability of the dispersions of micelles by preventing their aggregation due to charge repulsion.

To clarify the storage modulus dependency on $\varphi$ shown in Fig. 4B, $G^{\prime}$ of six dispersions of different concentrations of $7.5,10,12.5,15,20$, and 30 wt $\%$ at 10 and $40^{\circ} \mathrm{C}$ were compared. Fig. $5 \mathrm{~A}$ shows the total volume of micelles $\left(V_{m}\right)$ for each sample which increases linearly with concentration. As expected, the slope is higher for samples at $10^{\circ} \mathrm{C}$ than at $40{ }^{\circ} \mathrm{C}$ due to the hydration of the PNIPAM chains in the micellar core. A horizontal dashed line in Fig. $5 \mathrm{~A}$ shows that $V_{m}$ of the $12.5 \mathrm{wt} \%$ dispersion at $10{ }^{\circ} \mathrm{C}(2.2 \mathrm{~mL})$ is very close to $V_{m}$ of 30 wt\% dispersion at $40^{\circ} \mathrm{C}(2.1 \mathrm{~mL})$ (SI-table 2). According to the model presented in Fig. $4 \mathrm{~B}$ it is expected that these two samples display similar $G^{\prime}$ although their concentration and temperatures are very different. Fig. 5B shows that $G^{\prime}$ of the $12.5 \mathrm{wt} \%$ micellar dispersion at $10{ }^{\circ} \mathrm{C}(\sim 2600 \mathrm{~Pa})$ is indeed very close to $G^{\prime} \sim 2400 \mathrm{~Pa}$ found for the $30 \%$ sample at $40{ }^{\circ} \mathrm{C}$ (horizontal dashed line in Fig. 5B). 
A

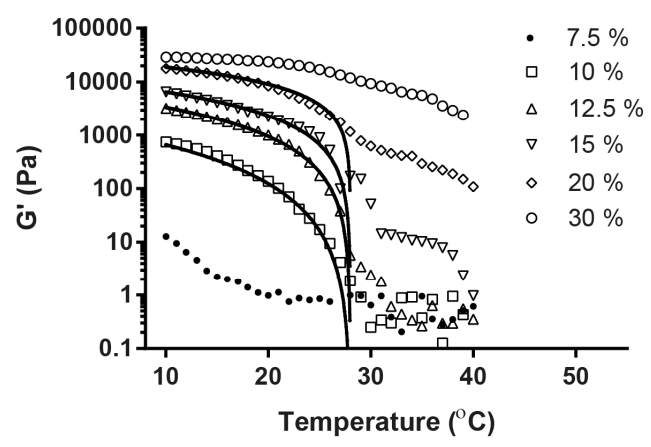

B

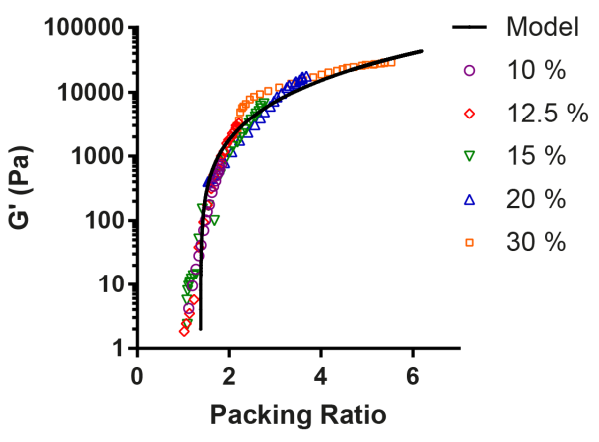

Figure 4: A) Storage moduli $\left(G^{\prime}\right)$ of micellar dispersions as a function of temperature and for different concentrations in PBS. The solid curves are the best fit of $G^{\prime} \sim\left(T_{c}-T\right)^{\gamma}$ to the data of samples with concentrations $10,12.5$, and $15 \%$. B) $G^{\prime}$ as a function of effective volume fraction $(\varphi)$ at different micellar concentrations at different temperatures. The solid curve is the best fit of $G^{\prime} \sim \varphi\left(\varphi-\varphi_{c}\right)$ with $\varphi_{c}=1.35$.

A

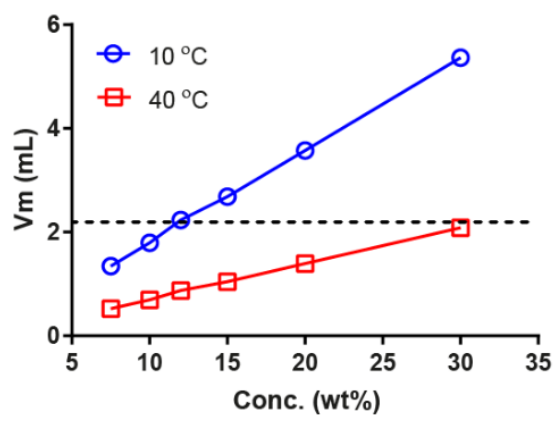

B

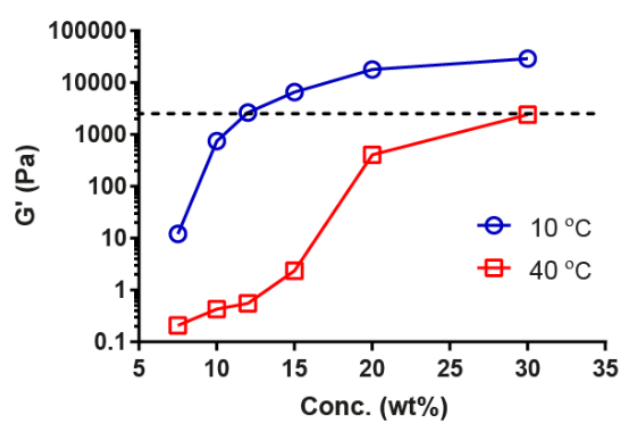

Figure 5: A) Total volume of micelles $\left(V_{m}\right)$ as a function of concentration at 10 and $40{ }^{\circ} \mathrm{C} . V_{m}$ for each dispersion was calculated based on the radius of micelles in a diluted micellar dispersion ( 3 $\mathrm{mg} \cdot \mathrm{mL}^{-1}$ ) (see SI-table 2). B) $G^{\prime}$ as a function of the concentration of micellar dispersion for the two temperatures. 
Yield stress is an important parameter for materials that need to undergo extrusion processes such as 3D-printing. Fig. 6A shows that a micellar dispersion with a concentration of 20 wt\% can be easily extruded through a nozzle even below the $T_{c}$. When the applied stress is larger than the yield stress of the material, the dispersion flows and can thus be extruded. However, after extrusion when the applied stress is below the yield stress the material retains its shape and shows solid-like behavior ${ }^{46}$. To investigate the yield behavior of the micellar dispersions, amplitude sweep rheology measurements were performed on micellar dispersions of 12.5 and 30 wt\% concentrations. In Fig. 6B, $G^{\prime}$ and $G^{\prime \prime}$ are shown as a function of applied stress for the two samples with the same packing fraction $\varphi \sim 2.2$ at different concentrations and temperatures. Both samples show similar $G^{\prime}$ and $G^{\prime \prime}$ values at low shear stresses. For both samples, with increasing stress, $G^{\prime}$ drops and becomes comparable to $G^{\prime \prime}(\sim 660 \mathrm{~Pa})$ at a stress of about $220 \mathrm{~Pa}$ (with an oscillation frequency of $1 \mathrm{~Hz}$ ). This point is known as the yield point of the material and the corresponding stress is called yield stress ${ }^{46}$. Therefore, the samples with similar packing ratio not only show similar $G^{\prime}$ and $G^{\prime \prime}$ but also display the same yield stresses.

A

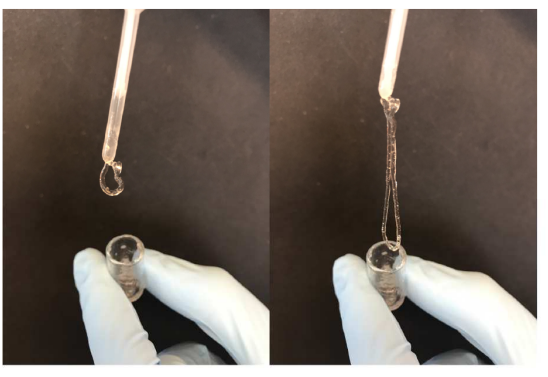

B

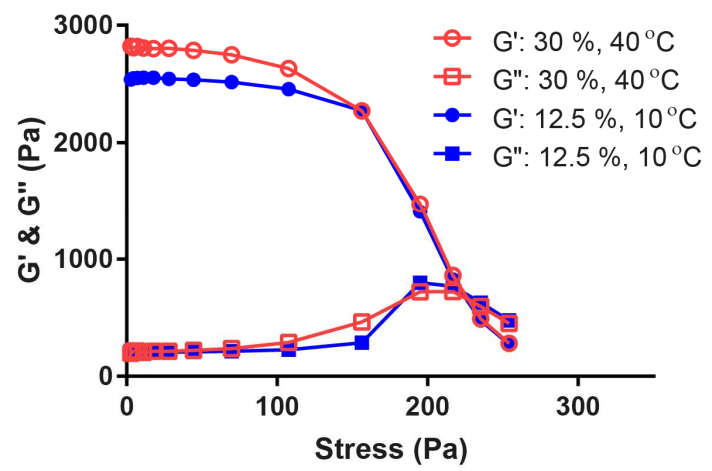

Figure 6: A) Extrusion of a micellar dispersion at a concentration of $20 \mathrm{wt} \%$ at $10^{\circ} \mathrm{C}$ through a $100 \mu \mathrm{L}$ tip of a positive displacement pipet. B) $G^{\prime}$ and $G^{\prime \prime}$ as a function of shear stress for two dispersions with the same packing fraction $(\varphi \sim 2.2)$.

\section{Conclusion}

In this study, the rheological properties of dense dispersions of flower-like micelles have been investigated. Temperature dependent change in the size of micelles resulted in formation of a colloidal glass with predictable storage modulus by changing the micelles' concentration from 7.5 to $30 \mathrm{wt} \%$ at temperatures from 10 to $40{ }^{\circ} \mathrm{C}$. When the storage modulus of different micellar dispersions at different concentrations and temperatures is 
plotted as a function of the effective volume fraction, the data points coincide onto a master curve. This indicates that the effective volume fraction of the micellar dispersions is the main parameter controlling their rheological properties. Furthermore, the volume fraction induced glass transition in the system is described by a theoretical model indicating a critical volume fraction of 1.35 , which is very close to the experimental value $(\varphi=1.2)$.

\section{Acknowledgments}

The Netherlands Organization for Scientific Research (NWO/VIDI 13457 and NWO/Aspasia 015.009.038) are acknowledged for funding. M.H. thanks prof. dr. A. Zaccone for a fruitful discussion. 


\section{References}

1. Najafi, M.; Hebels, E.; Hennink, W. E.; Vermonden, T., Poly( N -isopropylacrylamide): Physicochemical Properties and Biomedical Applications: Chemistry, Properties and Applications. In Temperature-Responsive Polymers: Chemistry, Properties, and Applications, Khutoryanskiy, V. V.; Georgiou, K. T., Eds. John Wiley \& Sons: 2018; pp 1-34.

2. Heskins, M.; Guillet, J. E., Solution Properties of Poly(N-isopropylacrylamide). Journal of Macromolecular Science: Part A - Chemistry 1968, 2 (8), 1441-1455.

3. Ashraf, S.; Park, H.-K.; Park, H.; Lee, S.-H., Snapshot of phase transition in thermoresponsive hydrogel PNIPAM: Role in drug delivery and tissue engineering. Macromolecular Research 2016, 24 (4), 297-304.

4.Cao, M.; Wang, Y.; Hu, X.; Gong, H.; Li, R.; Cox, H.; Zhang, J.; Waigh, T. A.; Xu, H.; Lu, J. R., Reversible Thermoresponsive Peptide-PNIPAM Hydrogels for Controlled Drug Delivery. Biomacromolecules 2019, 20 (9), 3601-3610.

5. Najafi, M.; Kordalivand, N.; Moradi, M. A.; van den Dikkenberg, J.; Fokkink, R.; Friedrich, H.; Sommerdijk, N.; Hembury, M.; Vermonden, T., Native Chemical Ligation for CrossLinking of Flower-Like Micelles. Biomacromolecules 2018, 19 (9), 3766-3775.

6. de Graaf, A. J.; Mastrobattista, E.; Vermonden, T.; van Nostrum, C. F.; Rijkers, D. T. S.; Liskamp, R. M. J.; Hennink, W. E., Thermosensitive Peptide-Hybrid ABC Block Copolymers Obtained by ATRP: Synthesis, Self-Assembly, and Enzymatic Degradation. Macromolecules 2012, 45 (2), 842-851.

7. Wei, H.; Cheng, S.-X.; Zhang, X.-Z.; Zhuo, R.-X., Thermo-sensitive polymeric micelles based on poly(N-isopropylacrylamide) as drug carriers. Progress in Polymer Science 2009, 34 (9), 893-910.

8. Topp, M. D. C.; Dijkstra, P. J.; Talsma, H.; Feijen, J., Thermosensitive Micelle-Forming Block Copolymers of Poly(ethylene glycol) and Poly(N-isopropylacrylamide). Macromolecules 1997, 30 (26), 8518-8520.

9. Le Bohec, M.; Banère, M.; Piogé, S.; Pascual, S.; Benyahia, L.; Fontaine, L., Sol-gel reversible metallo-supramolecular hydrogels based on a thermoresponsive double hydrophilic block copolymer. Polymer Chemistry 2016, 7 (44), 6834-6842.

10. de Graaf, A. J.; Azevedo Prospero dos, S., II; Pieters, E. H.; Rijkers, D. T.; van Nostrum, C. F.; Vermonden, T.; Kok, R. J.; Hennink, W. E.; Mastrobattista, E., A micelle-shedding thermosensitive hydrogel as sustained release formulation. Journal of Controlled Release 2012, 162 (3), 582-590. 
11. Wang, C.; Zhang, G.; Liu, G.; Hu, J.; Liu, S., Photo- and thermo-responsive multicompartment hydrogels for synergistic delivery of gemcitabine and doxorubicin. Journal of Controlled Release 2017, 259, 149-159.

12. Basu, A.; Xu, Y.; Still, T.; Arratia, P. E.; Zhang, Z.; Nordstrom, K. N.; Rieser, J. M.; Gollub, J. P.; Durian, D. J.; Yodh, A. G., Rheology of soft colloids across the onset of rigidity: scaling behavior, thermal, and non-thermal responses. Soft Matter 2014, 10 (17), 3027-3035.

13. Minami, S.; Suzuki, D.; Urayama, K., Rheological aspects of colloidal gels in thermoresponsive microgel suspensions: formation, structure, and linear and nonlinear viscoelasticity. Current Opinion in Colloid \& Interface Science 2019, 43, 113-124.

14. Guan, Y.; Zhang, Y., PNIPAM microgels for biomedical applications: from dispersed particles to 3D assemblies. Soft Matter 2011, 7 (14).

15. Wu, C.; Zhou, S.; Au-yeung, S. C. F.; Jiang, S., Volume phase transition of spherical microgel particles. Die Angewandte Makromolekulare Chemie 1996, 240 (1), 123-136.

16. Hirokawa, Y.; Tanaka, T., Volume phase transition in a non-ionic gel. AIP Conference Proceedings 1984, 107 (1), 203-208.

17. Anonymous, Nature 1972, 239, 488.

18. Torquato, S.; Truskett, T. M.; Debenedetti, P. G., Is Random Close Packing of Spheres Well Defined? Physical Review Letters 2000, 84 (10), 2064-2067.

19. Senff, H.; Richtering, W., Temperature sensitive microgel suspensions: Colloidal phase behavior and rheology of soft spheres. The Journal of Chemical Physics 1999, 111 (4), 17051711.

20. Cloitre, M.; Borrega, R.; Monti, F.; Leibler, L., Glassy Dynamics and Flow Properties of Soft Colloidal Pastes. Physical Review Letters 2003, 90 (6), 68303-68307.

21. Mason, T. G.; Weitz, D. A., Linear Viscoelasticity of Colloidal Hard Sphere Suspensions near the Glass Transition. Physical Review Letters 1995, 75 (14), 2770-2773.

22. Wang, T.; Jin, L.; Song, Y.; Li, J.; Gao, Y.; Shi, S., Rheological study on the thermoinduced gelation behavior of poly( $\mathrm{N}$-isopropylacrylamide-co-acrylic acid) microgel suspensions. Journal of Applied Polymer Science 2017, 134 (35), 45259.

23. Minami, S.; Watanabe, T.; Suzuki, D.; Urayama, K., Viscoelasticity of dense suspensions of thermosensitive microgel mixtures undergoing colloidal gelation. Soft Matter 2018, 14 (9), 1596-1607.

24. Minami, S.; Watanabe, T.; Suzuki, D.; Urayama, K., Rheological properties of suspensions of thermo-responsive poly( $\mathrm{N}$-isopropylacrylamide) microgels undergoing volume phase transition. Polymer Journal 2016, 48 (11), 1079-1086.

25. Gan, T.; Zhang, Y.; Guan, Y., In Situ Gelation of P(NIPAM-HEMA) Microgel Dispersion and Its Applications as Injectable 3D Cell Scaffold. Biomacromolecules 2009, 10 (6), 1410-1415. 
26. Romeo, G.; Fernandez-Nieves, A.; Wyss, H. M.; Acierno, D.; Weitz, D. A., TemperatureControlled Transitions Between Glass, Liquid, and Gel States in Dense p-NIPA Suspensions. Advanced Materials 2010, 22 (31), 3441-3445.

27. Es Sayed, J.; Lorthioir, C.; Perrin, P.; Sanson, N., PEGylated NiPAM microgels: synthesis, characterization and colloidal stability. Soft Matter 2019, 15 (5), 963-972.

28. Zaccone, A.; Terentjev, E. M., Disorder-Assisted Melting and the Glass Transition in Amorphous Solids. Physical Review Letters 2013, 110 (17), 178002-178007.

29. Lappala, A.; Zaccone, A.; Terentjev, E. M., Polymer glass transition occurs at the marginal rigidity point with connectivity $z^{*}=4$. Soft Matter 2016, 12 (35), 7330-7337.

30. Ulbrich, K.; Šubr, V.; Strohalm, J.; Plocová, D.; Jelínková, M.; Říhová, B., Polymeric drugs based on conjugates of synthetic and natural macromolecules: I. Synthesis and physicochemical characterisation. Journal of Controlled Release 2000, 64 (1), 63-79.

31. Boere, K. W. M.; Soliman, B. G.; Rijkers, D. T. S.; Hennink, W. E.; Vermonden, T., Thermoresponsive Injectable Hydrogels Cross-Linked by Native Chemical Ligation. Macromolecules 2014, 47 (7), 2430-2438.

32. Boere, K. W. M.; van den Dikkenberg, J.; Gao, Y.; Visser, J.; Hennink, W. E.; Vermonden, T., Thermogelling and Chemoselectively Cross-Linked Hydrogels with Controlled Mechanical Properties and Degradation Behavior. Biomacromolecules 2015, 16 (9), 2840-2851.

33. Soga, O.; van Nostrum, C. F.; Ramzi, A.; Visser, T.; Soulimani, F.; Frederik, P. M.; Bomans, P. H. H.; Hennink, W. E., Physicochemical Characterization of Degradable Thermosensitive Polymeric Micelles. Langmuir 2004, 20 (21), 9388-9395.

34. BioScience ${ }^{\mathrm{TM}}$ U. Reactive Tag Viscosity Probes. https://ursabioscience.com/technology/viscosity-probes/conjugatable-viscosity-probes. 35. Suhina, T.; Weber, B.; Carpentier, C. E.; Lorincz, K.; Schall, P.; Bonn, D.; Brouwer, A. M., Fluorescence Microscopy Visualization of Contacts Between Objects. Angewandte Chemie International Edition 2015, 54 (12), 3688-3691.

36. Matyjaszewski, K.; Xia, J., Atom Transfer Radical Polymerization. Chemical Reviews 2001, 101 (9), 2921-2990.

37. Smithenry, D. W.; Kang, M.-S.; Gupta, V. K., Telechelic Poly(N-isopropylacrylamide): Polymerization and Chain Aggregation in Solution. Macromolecules 2001, 34 (24), 8503 8511.

38. Ganachaud, F.; Monteiro, M. J.; Gilbert, R. G.; Dourges, M.-A.; Thang, S. H.; Rizzardo, E., Molecular Weight Characterization of Poly(N-isopropylacrylamide) Prepared by Living Free-Radical Polymerization. Macromolecules 2000, 33 (18), 6738-6745.

39. Dawson, P. E.; Muir, T. W.; Clark-Lewis, I.; Kent, S. B., Synthesis of proteins by native chemical ligation. Science 1994, 266 (5186), 776-779. 
40. de Graaf, A. J.; Boere, K. W.; Kemmink, J.; Fokkink, R. G.; van Nostrum, C. F.; Rijkers, D. T.; van der Gucht, J.; Wienk, H.; Baldus, M.; Mastrobattista, E.; Vermonden, T.; Hennink, W. E., Looped structure of flowerlike micelles revealed by $1 \mathrm{H}$ NMR relaxometry and light scattering. Langmuir 2011, 27 (16), 9843-9848.

41. Martínez-Jothar, L.; Doulkeridou, S.; Schiffelers, R. M.; Sastre Torano, J.; Oliveira, S.; van Nostrum, C. F.; Hennink, W. E., Insights into maleimide-thiol conjugation chemistry: Conditions for efficient surface functionalization of nanoparticles for receptor targeting. Journal of Controlled Release 2018, 282, 101-109.

42. Loutfy, R. O., Fluorescence probes for polymer free-volume. In Pure and Applied Chemistry, 1986; Vol. 58, p 1239.

43. Jin, H.; Liang, M.; Arzhantsev, S.; Li, X.; Maroncelli, M., Photophysical Characterization of Benzylidene Malononitriles as Probes of Solvent Friction. The Journal of Physical Chemistry B 2010, 114 (22), 7565-7578.

44. Onuki, A., Phase Transition Dynamics. Cambridge University Press: Cambridge, 2002. 45. Stanley, H. E., Introduction to phase transitions and critical phenomena. Oxford University Press: New York, 1971.

46. Rahmani, Y.; Habibi, M.; Javadi, A.; Bonn, D., Coiling of yield stress fluids. Physcal Review E Covering Statistical, Nonlinear, Biological, and Soft Matter Physics 2011, 83, 56327-56331. 


\section{Supporting information:}

\section{Static and Dynamic Light Scattering (SLS and DLS)}

Static light scattering (SLS) and dynamic light scattering (DLS) were conducted as a function of the detection angle $\Theta$. In SLS measurement, the scattering intensities for sample $\left(I_{s}\right)$, toluene (ltol), and PBS as solvent ( $\left(l_{0}\right)$ were measured as a function of the scattering angle $\Theta$. Furthermore the dark current $I_{\text {dark }}$ (detector intensity with laser switched off) was measured. The obtained intensities were converted into Rayleigh scattering $(R)$ to obtain absolute scattering of the sample according to equation 1 :

$$
R(\Theta)=\frac{I_{S}(\Theta)-I_{0}(\Theta)}{I_{\text {tol }}(\Theta)-I_{\text {dark }}} \frac{n_{S}^{2}}{n_{\text {tol }}^{2}} R_{\text {tol }}
$$

In which $n_{\text {tol }}=1.494$ and $n_{s}=1.332$ are the refractive index of the reference and solvent, respectively, and $R_{t o l}$ is the known absolute Rayleigh scattering for toluene at $632.8 \mathrm{~nm}$ wavelength. For $R_{\text {tol }}$ we used $1.0210^{-3} \mathrm{~m}^{-1} 1$, Then the scattering angle $\Theta$ was converted into the wave vector $q$ based on equation 2 :

$$
q=\frac{4 \pi \mathrm{n}_{s} \sin \frac{\Theta}{2}}{\lambda}
$$

where $\lambda$ is the wavelength in the vacuum.

- Therefore, $R_{g}$ was obtained according to Guinier ${ }^{2}$ by plotting $\ln (R)$ as a function of $q^{2}$ in which the slope is equal to: $\frac{R_{g}^{2}}{3}$ (results are shown in table 1 )

- Hydrodynamic radius $\left(R_{h}\right)$ was obtained by DLS. Decay rate $\Gamma$ extracted from the second order cumulant was plotted as a function of $q^{2}$. For monodisperse particles this should result in a straight line with a slope equals to the diffusion coefficient $D$ and from that $R_{h}$ was obtained using equation 3: (results are shown in table 1)

$$
D=\frac{\mathrm{kT}}{6 \pi \eta R_{h}}
$$

- To obtain $M_{w}$ absolute scattering intensity $R$ was plotted as a function of $q$ and extrapolated to $q=0$. The absolute scattering $R$ should equal to: (results are shown in table 1) 
(4)

$$
R=K_{r} C M_{w} \mathrm{~S}(\mathrm{q}) \mathrm{P}(\mathrm{qR})
$$

At low concentration, the structure factor $S(q) \approx 1$ and at $q \rightarrow 0$ the form factor was considered $\mathrm{P}(\mathrm{qR})=1$, therefore, $R(q \rightarrow 0)=K_{r} C, \mathrm{M}_{\mathrm{w}}$ where $K_{r}$ an optical constant (equation 5 ) and $C$ the particle concentration in $\mathrm{kg} \cdot \mathrm{m}^{-3}$.

$$
K_{r}=\frac{4 n_{S}^{2} \pi^{2}}{\lambda^{4} N_{A v}}\left(\frac{d n}{d c}\right)^{2}
$$

SI-Table 1: Characteristics of core crosslinked flower-like micelles measured at 10 and $40{ }^{\circ} \mathrm{C}$. Reproduced with permission from ref ${ }^{3}$ (https://doi.org/10.1021/acs.biomac.8b00908). Permission for reusing this data should be directed to the ACS.

\begin{tabular}{cccccc}
\hline $\mathbf{T}\left({ }^{\circ} \mathrm{C}\right)$ & $\mathbf{R}_{\mathrm{g}}(\mathrm{nm})^{\mathrm{a}}$ & $\mathbf{R}_{\mathrm{h}}(\mathrm{nm})^{\mathrm{b}}$ & $\mathbf{R}_{\mathrm{g}} / \mathbf{R}_{\mathrm{h}}$ & $\begin{array}{c}\mathbf{M}_{\mathrm{w} \text { (mic.) }} \\
\left(\mathbf{1 0 ^ { 6 }} \mathrm{Da}\right)\end{array}$ & $\mathbf{N}_{\mathrm{agg}}{ }^{\mathrm{d}}$ \\
\hline $\mathbf{1 0}$ & $46.0 \pm 0.8$ & $48.2 \pm 0.9$ & $0.95 \pm 0.00$ & $16.3 \pm 0.4$ & $430 \pm 10$ \\
\hline $\mathbf{4 0}$ & $30 \pm 0.8$ & $35.4 \pm 0.4$ & $0.85 \pm 0.01$ & $14.8 \pm 0.2$ & $381 \pm 10$ \\
\hline
\end{tabular}

\section{Calculation of Effective Volume Fraction ( $\varphi$ )}

$V_{t}=$ Micelles $\left.(\mathrm{mg}) \times 0.83 \mathrm{~cm} \cdot \mathrm{g}^{-1}(\text { partial specific volume of PNIPAM })^{1}\right)+$ PBS $(\boldsymbol{m L})$

Number of micelles $=\frac{\text { Micelles }(\text { gr })}{N_{A} \times M_{w} \text { micelle }}$, where $N_{A}$ is Avogadro constant and $M_{w}$ is the micelle molecular weight $15.55 \times 10^{6} \mathrm{kDa}$ (as the average molecular weight of micelles at 10 and $40^{\circ} \mathrm{C}$ was considered)

$V_{m}=$ number of micelles $\times$ volume of micelles at the corresponding temperature 
SI-Table 2: The summary of results for calculation of $\varphi . T_{C}$ is $28^{\circ} \mathrm{C}$.

\begin{tabular}{|c|c|c|c|c|c|c|c|c|}
\hline \multirow{2}{*}{$\begin{array}{l}\text { Sample } \\
\text { conc. } \\
\text { (wt\%) }\end{array}$} & \multirow[t]{2}{*}{$\begin{array}{c}\text { Micelles } \\
\text { (mg) }\end{array}$} & \multirow[t]{2}{*}{$\begin{array}{l}\text { PBS } \\
(\mathrm{mL})\end{array}$} & \multirow{2}{*}{$\begin{array}{c}\text { Sample } \\
\text { volume } \\
\left(V_{t}\right) \\
(\mathrm{mL})\end{array}$} & \multirow{2}{*}{$\begin{array}{c}\text { Number } \\
\text { of } \\
\text { micelles } \\
\left(\times 10^{15}\right)\end{array}$} & \multicolumn{2}{|c|}{$\begin{array}{l}\text { Volume of micelles } \\
\qquad\left(V_{m}\right)(m L)\end{array}$} & \multicolumn{2}{|c|}{$\boldsymbol{\varphi} \operatorname{or}\left(\frac{V_{t}}{V_{m}}\right)$} \\
\hline & & & & & $\begin{array}{l}\text { Below } \\
T_{c}\end{array}$ & $\begin{array}{l}\text { Above } \\
\qquad T_{c}\end{array}$ & $\begin{array}{l}\text { Below } \\
\qquad T_{c}\end{array}$ & $\begin{array}{c}\text { Above } \\
T_{c}\end{array}$ \\
\hline 7.5 & 75 & 0.925 & 0.987 & 2.89 & 1.34 & 0.52 & 1.36 & 0.53 \\
\hline 10 & 100 & 0.900 & 0.983 & 3.86 & 1.79 & 0.69 & 1.82 & 0.7 \\
\hline 12.5 & 125 & 0.875 & 0.979 & 4.82 & 2.23 & 0.87 & 2.28 & 0.88 \\
\hline 150 & 150 & 0.850 & 0.975 & 5.79 & 2.68 & 1.04 & 2.75 & 1.07 \\
\hline 200 & 200 & 0.800 & 0.966 & 7.72 & 3.57 & 1.39 & 3.7 & 1.43 \\
\hline 300 & 300 & 0.700 & 0.949 & 11.6 & 5.36 & 2.08 & 5.65 & 2.19 \\
\hline
\end{tabular}




\section{Figures}

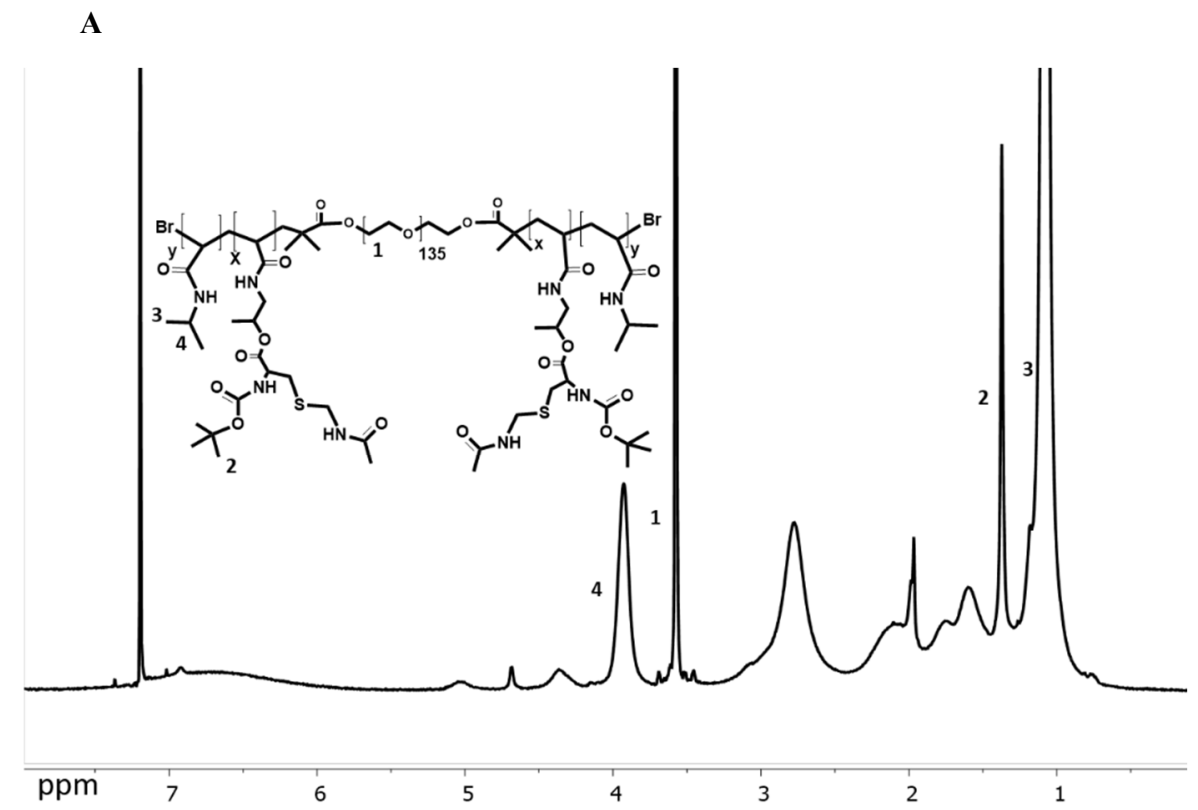

B

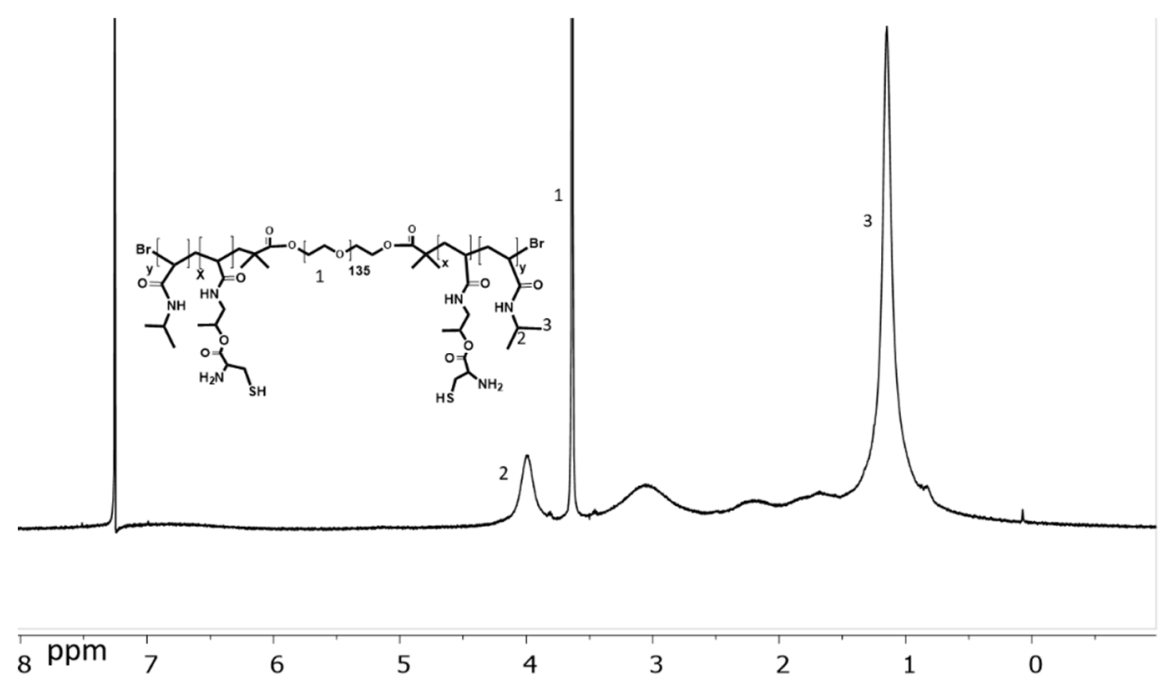

SI-Figure 1: ${ }^{1} \mathrm{H}-\mathrm{NMR}$ spectra of $\mathrm{A}$ ) protected PNC, B) PNC in $\mathrm{CDCl}_{3}$. 


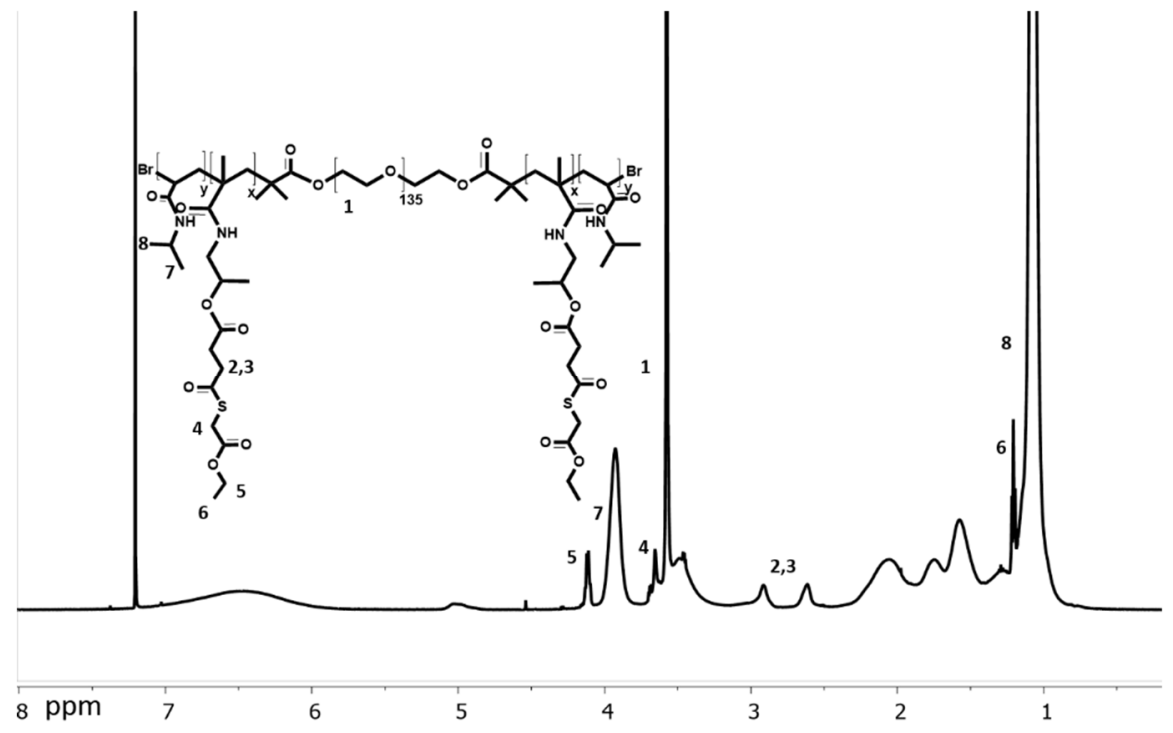

SI-Figure 2: ${ }^{1} \mathrm{H}-\mathrm{NMR}$ spectrum of PNE in $\mathrm{CDCl}_{3}$.

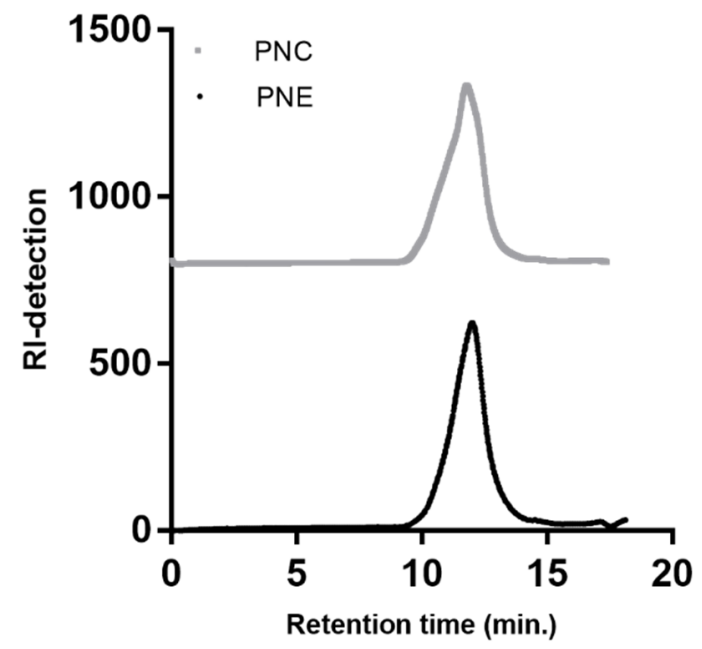

SI-Figure 3: GPC chromatograms of PNC and PNE in DMF containing $10 \mathrm{mM} \mathrm{LiCl.}$ 


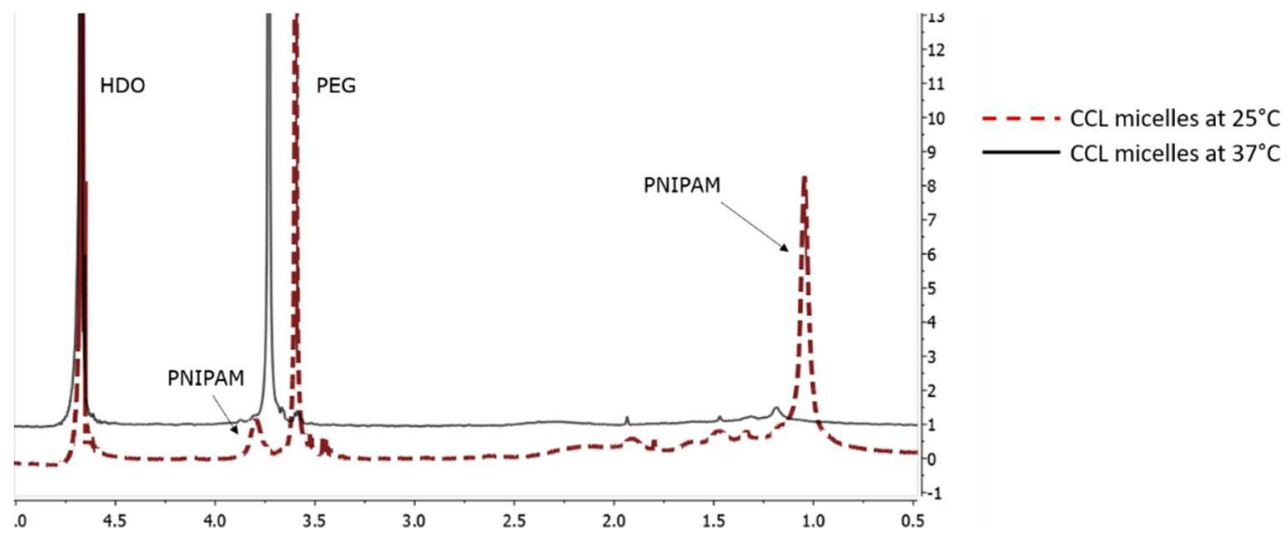

Figure 4: ${ }^{1} \mathrm{H}-\mathrm{NMR}$ spectra of the core-crosslinked micelles in $\mathrm{D}_{2} \mathrm{O}$ below and above LCST of the polymers. At temperature above LCST, the intensity of PNIPAM signal drastically dropped while PEG signals are still present, which indicates the exposure of hydrophilic PEG in the corona of micelles. The peak shifts are due to the change in temperature. Reproduced with permission from reference ${ }^{3}$ (https://doi.org/10.1021/acs.biomac.8b00908).

1. Wu, H., Correlations between the Rayleigh ratio and the wavelength for toluene and benzene. Chemical Physics 2010, 367 (1), 44-47.

2. Guinier, A.; Fournet, G., Small-Angle Scattering of X-Rays. John Wiley and Sons, Inc., New York 1955.

3. Najafi, M.; Kordalivand, N.; Moradi, M. A.; van den Dikkenberg, J.; Fokkink, R.; Friedrich, H.; Sommerdijk, N.; Hembury, M.; Vermonden, T., Native Chemical Ligation for Cross-Linking of Flower-Like Micelles. Biomacromolecules 2018, 19 (9), 3766-3775.

4. Sommer, C.; Pedersen, J. S.; Stein, P. C., Apparent Specific Volume Measurements of Poly(ethylene oxide), Poly(butylene oxide), Poly(propylene oxide), and Octadecyl Chains in the Micellar State as a Function of Temperature. The Journal of Physical Chemistry B 2004, 108 (20), 6242-6249. 



\section{Chapter 7}

Summary and perspectives 


\section{Summary}

Advancements in polymer chemistry and pharmaceutical sciences have resulted in the rationale design of nanosized drug delivery systems ${ }^{1-4}$. Polymeric micelles (PM) with a diameter ranging from 10-100 $\mathrm{nm}$ with a core-shell structure can be formed from amphiphilic block copolymers ${ }^{5-7}$. The hydrophobic core and hydrophilic shell, together with tunable properties e.g. chemical conjugation of a drug to the micellar core and a targeting ligand to the shell, make polymeric micelles well suited for drug delivery applications ${ }^{8}$ and a great variety of micellar systems are under clinical evaluations ${ }^{9,10}$. Hydrogels are another type of well-known polymeric materials that have been extensively studied for drug delivery applications. Hydrogels are three-dimensional (3D) networks of chemically or physically crosslinked hydrophilic polymers that absorb large amounts of water while maintaining their structure ${ }^{11,12}$. In recent years, micellar-hydrogel systems that combine unique features of both micelles and hydrogels have gained increasing interest. In such systems, among others, hydrogels are converted into micelles upon stimuli or simply by dilution in the surrounding aqueous medium ${ }^{13}, 14$. This thesis reports research on the development of core crosslinked flower-like micelles, on their in-vivo behavior in comparison with star-like micelles, and on their formation upon enzymatic degradation of a macroscopic hydrogel. Chapter 1 introduces the main concepts investigated in this thesis. In this chapter, polymeric micelles and the role of micelle crosslinking in improving micellar stability are described. The stealth effect of poly(ethylene glycol) (PEG) in improving the circulation kinetics of nanoparticles is described and the accelerated blood clearance ( $A B C$ phenomenon) of subsequent doses of PEGylated therapeutics and particles is discussed ${ }^{15}$. Furthermore, stimuli-responsive hydrogels and their potential as local drug delivery systems are disputed.

Chapter 2 gives an overview of the physicochemical properties and biomedical applications of poly( $N$-isopropylacrylamide) (PNIPAM). PNIPAM is considered an interesting polymer to prepare materials for biomedical and pharmaceutical applications because of its lower critical solution temperature (LCST) behavior around $32{ }^{\circ} \mathrm{C}$ in aqueous media ${ }^{16}$. The use of this thermosensitive polymer has been widely reported in the literature for several applications such as PNIPAM functionalized substrates in cell culture ${ }^{17-19}$, bioseparation ${ }^{20 \text {, }}$ ${ }^{21}$, biosensing ${ }^{22,} 23$ and drug delivery applications ${ }^{24}$. Incorporation of functional groups such as $\mathrm{pH}$-sensitive ${ }^{25}$-, photo-sensitive ${ }^{26}$ or enzyme hydrolyzable ${ }^{27}$ groups in the PNIPAM structure allows engineering of responsive drug carriers ${ }^{28,29}$. This chapter discusses the copolymerization of NIPAM with functional monomers using several polymerization techniques including atom transfer radical polymerization (ATRP) $)^{30}$, reversible addition-fragmentation chain-transfer polymerization (RAFT), and free radical 
polymerization (FRP). Self-assembly of the synthesized polymers into organized structures, e.g. micelles and hydrogels are described, together with several crosslinking methods to tailor the stability of these materials. For instance, matrix metalloproteinase (MMP) responsive PNIPAM based micelles as a peptide-polymer hybrid system and their potential for enzyme-triggered drug delivery are described ${ }^{27}$. It is further discussed that the selfassembly of ABA tri-block copolymers of PNIPAM (A-block) and PEG (B-block) results in flower-like micelles in aqueous media ${ }^{31}$. This concept was exploited in a previous $\mathrm{PhD}$ thesis of our department for the formulation of a micelle-shedding PNIPAM based hydrogel for the sustained release of an anti-cancer drug ${ }^{13,32}$.

To improve stability of PNIPAM-based materials under biological conditions, physical crosslinking (by hydrogen bonding) of PNIPAM at a temperature above LCST has been combined with several chemical crosslinking methods ${ }^{33}$. In the case of PNIPAM based hydrogels, in-situ crosslinking methods allow designing of injectable hydrogels. Native chemical ligation as a crosslinking method for the formation of injectable hydrogels is highlighted in this chapter ${ }^{34,35}$. In Chapter 3, native chemical ligation (NCL) ${ }^{35}$ is employed as a selective and efficient method for core crosslinking of flower-like micelles ${ }^{36}$. This reaction involves coupling of an $\mathrm{N}$-terminal cysteine to a thioester to form a thioester-linked intermediate product that after spontaneous S-N-acyl rearrangement yields an amide bond. This crosslinking method benefits from proceeding catalyst-free, at ambient temperature, in aqueous media, and at physiological $\mathrm{pH}$. Moreover, after $\mathrm{NCL}$, free thiol moieties remain available, which can be exploited for conjugation of desirable molecules (a dye or drug) via e.g. a disulfide bond, Michael addition, or thiol-ene reactions. Two complementary ABA block copolymers containing A blocks of NIPAM and either $\mathrm{N}$-(2hydroxypropyl)methacrylamide-ethylthioglycolate succinic acid (HPMA-ETSA) or $\mathrm{N}$-(2hydroxypropyl)methacrylamide-cysteine (HPMA-Cys) and a B block of PEG were synthesized

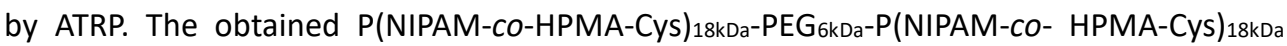

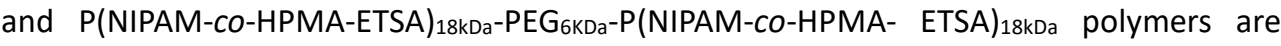
abbreviated as PNC and PNE, respectively. Mixing the aqueous solutions of these polymers and raising the temperature above the LCST of the PNIPAM blocks resulted in the selfassembly of polymers into flower-like micelles with a PEG shell, and a PNIPAM based core. Subsequently, native chemical ligation in the micellar core yielded stabilized micellar structures with a Z-average of $\sim 65 \mathrm{~nm}$ at body temperature. The ratio between the functional groups (cysteines and thioesters) in the micellar core could be tuned by simply adjusting the ratio between the polymers before micellization yielding a defined number of functional groups available for conjugation of dye or drug molecules. A comprehensive structural study of micelles using Cryo-TEM and static light scattering (SLS) confirmed the formation of uniform and spherical micelles. Interestingly, we observed dark patches in the 
tomographic view representing more dense areas in the micelles, which coincide with the relatively high content of HPMA-Cys/ETSA close to the PEG chain as revealed by a polymerization kinetics study. Furthermore, we demonstrated that these micelles could be taken up by human epithelial cervix carcinoma cells (HeLa cells), while good cell viability was retained. Although the cellular uptake was rather low, these results demonstrate the potential of these core crosslinked flower-like micelles as a drug carrier. Whereas several reports have shown the application of flower-like micelles as nanocarriers ${ }^{37,38}$, the possible advantages of flower-like micelles over star-like micelles for pharmaceutical applications have not been described yet. Therefore, in Chapter 4, circulation kinetics and biodistribution of the flower-like micelles introduced in chapter 3 were compared with star-like micelles. The star-like micelles were composed of two complementary $A B$ block copolymers; the $A$ blocks had same size and composition as the $A$ blocks in flower-like micelles and the $B$ block was methoxy polyethylene glycol (mPEG) ( $\mathrm{Mn}_{\mathrm{n}} 3 \mathrm{kDa}$ ). Star-like micelles core crosslinked by native chemical ligation were prepared according to the same micellization method as used for the flower-like micelles in chapter 3 . The flower- and star-like micelles displayed Zaverage of $\sim 65$ and $83 \mathrm{~nm}$, respectively. SLS characterization of the obtained micelles showed aggregation numbers ( $\mathrm{N}_{a g g}$ ) of $\sim 400$ and 1700 for flower- and star-like micelles, respectively. The lower $\mathrm{N}_{\text {agg }}$ in flower-like micelles in comparison with star-like micelles is due to the loop conformation of PEG. In this conformation, PEG chains occupy more surface area compared to linear PEG in star-like micelles, which led to fewer chains fitting in one micelle ${ }^{31}$. Consequently, the surface area per PEG chain (SA/PEG) for flower- and star-like micelles was calculated to be $\sim 37$ vs. $12 \mathrm{~nm}^{2}$, respectively. The low value of SA/PEG for starlike micelles $\left(\sim 12 \mathrm{~nm}^{2}\right)$ indicates that the surface has a high PEG grafting density and PEG chains are in a highly stretched "brush" conformation ${ }^{39,40}$. The large value of SA/PEG for flower-like indicates low PEG grafting intensity. In such structure, PEG chains show low flexibility in a loop conformation meaning they are highly stretched in a "mushroom" conformation $^{31}$. The circulation kinetics of flower- or star-like micelles were studied in immunocompetent BALB/c mice upon intravenous (i.v.) injection. Flower-like micelles exhibited significantly longer circulation half-life compared to star-like micelles upon the first injection ( $\mathrm{t}_{1 / 2} \sim 18.6 \pm 3.1$ vs. $10.7 \pm 0.7 \mathrm{~h}$ ). The longer circulation kinetics of the flower-like micelles can be ascribed to the reduced flexibility of PEG in the loop structure. To investigate the effect of PEG conformation on the ABC phenomenon, seven days after the first injection, each of the above groups was divided into two groups receiving another i.v. injection of either flower- or star-like micelles. Upon the second administration, accelerated blood clearance was observed after the injection of star-like micelles in mice that received star( $\mathrm{t}_{1 / 2}$ of $\sim 2.3 \pm 0.3 \mathrm{~h}$ ) or flower-like micelles as first injection (in 2 out of 3 mice no micelles were detected in the circulation after 24 hours). On the other hand, injection of flower-like 
micelles, whether applied as a first, second of both injections, resulted in similar in vivo kinetics ( $\mathrm{t}_{1 / 2} \sim 14.4 \pm 1.1$ and $15.4 \pm 2.2 \mathrm{~h}$ ). An ELISA study revealed binding of commercial antiPEG antibodies (IgM and IgG) to star-like micelles but not to flower-like micelles. Determination of anti-PEG IgM titers in the blood collected 24 hours after the second injection showed that indeed anti-PEG IgM antibodies are present upon injection of both type of micelles. Taken together, different binding of anti-PEG IgM to star-like micelles compared to flower-like micelles was observed, which is probably due to the presence of methoxy end group in the linear PEG present in the corona of star-like micelles ${ }^{41,}{ }^{42}$. Moreover, biodistribution of micelles was studied after the first and the second injections by measuring the fluorescence intensity of micelles in excised organs ( $24 \mathrm{~h}$ after injections). All groups showed similar results and micelles showed accumulation in liver and spleen as main clearance organs. These findings suggest that introducing PEG in a looped rather than a linear/brush conformation on nanoparticles could be used to enhance circulation time and reduces the occurrence of the $A B C$ phenomenon upon repeated injections.

In chapter 5, an enzyme responsive micellar hydrogel is introduced. Flower-like micelles (introduced in chapter 3 ) were prepared with a slight excess of PNE compared to PNC (molar ratio 3:2). The obtained micelles were linked together by pentablock copolymers of P(NIPAM-Co-HPMA-Cys)-PEG-Peptide-PEG-P(NIPAM-co-HPMA-Cys) (Pep-NC) to yield a hydrogel. The peptide midblock consisted of either $L$ or $D$ amino acids (lysine-glycineproline-glutamine-isoleucine-phenylalanine-glycine-glutamine-lysine (Lys-Gly-Pro-Gln-GlyIle-Phe-Gly-Gln-Lys)) (L-Pep-NC or D-Pep-NC), of which the L-amino acid sequence is a substrate for matrix metalloproteases 2 and 9 (MMPs 2 and 9). Native chemical ligation between thioester functionalities in the micellar core and cysteine functionalities in the $\mathrm{D} / \mathrm{L}$ Pep-NC polymers resulted in a hydrogel (D or L-HyMic) that was confirmed by increasing storage modulus $\left(G^{\prime}\right)$ from $\sim 600$ to $\sim 1300 \mathrm{~Pa}$ and a decrease in tan $\delta$ from $\sim 0.2$ to $\sim 0.06$. Incubation of both types of hydrogels with collagenase type IV (a model for MMPs) showed that the gels linked with L-Pep-NC (L-HyMic) degraded in 7-45 days upon exposure to collagenase in a concentration-dependent manner, while the gel linked by D-Pep-NC (DHyMic) remained intact even after 2 months. These observations demonstrate that degradation is indeed triggered by the enzymatic cleavage of L-Pep-NC. Additionally, the hydrogel supernatant was investigated by dynamic light scattering (DLS), which confirmed the presence of nanosized particles with a size close to the that of micelles before linking into the gel ( 120 vs. $93 \mathrm{~nm}$ ). Moreover, internalization of the released core-crosslinked micelles by Hela cells was observed. These results show the potential for HyMic for triggered release of CCL micelles for intracellular drug delivery in tissues with upregulation of MMPs. The core crosslinked flower-like micelles (introduced in chapter 3 ) display a radius of $\sim 35$ $\mathrm{nm}$ above the LCST, which rises to $\sim 48 \mathrm{~nm}$ by decreasing the temperature below the LCST 
due to hydration of the PNIPAM core. The change in the size as a function of temperature displays an inflection point at $\sim 28^{\circ} \mathrm{C}$. In chapter 6 , the rheological characterization of dense dispersions of core crosslinked flower-like micelles is investigated. Micelle dispersions displayed solid-like behavior below $28{ }^{\circ} \mathrm{C}$ with a $G^{\prime}$ depending on the sample concentration e.g. $\sim 700,3000$, and $6000 \mathrm{~Pa}$ at concentrations of 10, 12.5, and 15 wt\%, respectively, with a $\tan \delta$ of $\sim 0.05$. The effective volume fraction $\varphi=V_{m} / V_{t}$ was calculated for each concentration and temperature $\left(V_{m}\right.$ is the total micelles' volume at a specific concentration and $V_{t}$ is the sample volume). $\varphi$ was used to quantify and compare micelle packing in different samples at different concentrations and temperatures. We observed that the soft nature of micelles and their compressibility allow them to pack well above the random close packing fraction for hard spheres $(\Phi \approx 0.64)^{43,44}$, which resulted in glassy dynamics below $28^{\circ} \mathrm{C}$. When the storage moduli of different micellar dispersions at different concentrations and temperatures were plotted as a function of the effective volume fraction $(\varphi)$, the data points coincided onto a master curve. This master curve demonstrated that $\varphi$ of the micellar dispersions is the main parameter controlling their rheological properties. The experimental data were described with the theoretical disordered lattice model ${ }^{45} G^{\prime} \sim \varphi\left(\varphi-\varphi_{c}\right)$ and yielded the critical volume fraction of $\varphi_{c}=1.35$ close to the experimental value of $\varphi=1.2$. It was shown that two dispersions with equal $\varphi \approx 2.2$ at different temperatures $\left(10\right.$ and $\left.40^{\circ} \mathrm{C}\right)$, different micelles' radius ( $\sim 35$ and $48 \mathrm{~nm}$ ), and different concentrations (12.5 and $30 \mathrm{wt} \%)$ displayed similar $G^{\prime} \quad(\sim 2500$ $\mathrm{Pa})$ and yield stress $(\sim 220 \mathrm{~Pa})$ due to equal effective volume fraction. In contrast to previously studied PNIPAM microgel dispersions ${ }^{46}$, neither precipitation nor gelation of the micelles above the LCST was observed, most likely due to the presence of PEG on the micelles' corona.

\section{Discussion and Perspectives}

\subsection{Polymer choice and polymerization techniques}

Degradability of polymers in physiological conditions is mostly required for biomedical and pharmaceutical applications, and PNIPAM as such does not fulfill this requirement. The core crosslinked micelles described in this thesis have several ester bonds in the polymer backbone (between PEG and PNIPAM blocks) and in the crosslinked domains (originating from the HPMA-Cys and HPMA-ETSA monomers). The cleavage of these ester bonds could eventually lead to the slow degradation of micelles into polymer blocks with a molecular weight below the threshold of renal clearance, which is about $30-50 \mathrm{kDa}{ }^{47}$. However, the insolubility of PNIPMA blocks at body temperature is an issue and two approaches can be used to tackle this. The first approach is replacing PNIPAM by a biodegradable 
thermosensitive polymer such as copolymers of $\mathrm{N}$-(2-hydroxypropyl)methacrylamide mono- and dilactate (HPMAm(lac $\left.)_{n}\right)^{40,48}$. Poly (HPMA-(lac) $)_{n}$ ) polymers display a tunable cloud point (CP) by varying the molar ratio of HPMA-lac ${ }_{1}$ and HPMA-lac 2 . Notably, in aqueous medium hydrolysis of lactate acid groups in the side chains results in conversion of this insoluble polymer into PHPMAm, which is a water-soluble polymer ${ }^{40,48}$. The second approach is copolymerization of NIPAM with a degradable monomer such as dimethyl- $\gamma$ butyrolactone acrylate (DBA) $)^{49}$ or HPMA-lac ${ }^{50}$ to obtain a bioresorbable PNIPAM based polymer. In this way, hydrolysis of HPMA-lac or DBA moieties increases the overall hydrophilicity of the copolymer and consequently increases the LCST of the polymer above body temperature ${ }^{40,51}$.

The first approach was not employed in this study since HPMA-lac, for largely unknown reasons, cannot be polymerized with ATRP. On the other hand, free radical polymerization as a successful method for synthesis of PHPMA-(lac) $)_{n}$ could not be employed since this would not results in a well-defined tri-block copolymer for formulation of flower-like micelles. To obtain a bioresorbable polymer, RAFT polymerization was employed for copolymerization of NIPAM, DBA, and HPMA-Cys using a bifunctional PEG RAFT agent. The polymerization resulted in an ABA copolymer of P(NIPAM-Co-HPMA-Cys-Co-DBA)(25kDa)-

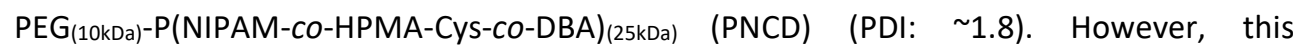
polymerization method was not compatible with the HPMA-ETSA monomer and resulted in polymer with a very high PDI ( 2.8). This high PDI is likely caused by the presence of a trace amount of thiol groups remaining from the synthesis of HPMA-ETSA which can act as a chain transfer agent during polymerization. Therefore, HPMA-ETSA was replaced by $\mathrm{N}$ hydroxysuccinimide (NAS) $)^{51}$ and P(NIPAM-CO-NAS-CO-DBA) (25kDa)-

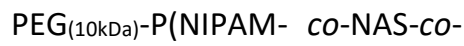
$\mathrm{DBA})_{(25 \mathrm{kDa})}$ (PNND) (PDI: 1.4) was obtained. Four types of PNCD and PNND polymers with different ratios of DBA were synthesized (molar ratio of DBA was $2.5,5,7$, and $10 \%$ of the total monomers). The obtained polymers (PNND and PNCD) were formulated into hydrogels at total polymer concentration of 15 wt\%. We observed that the obtained hydrogels were

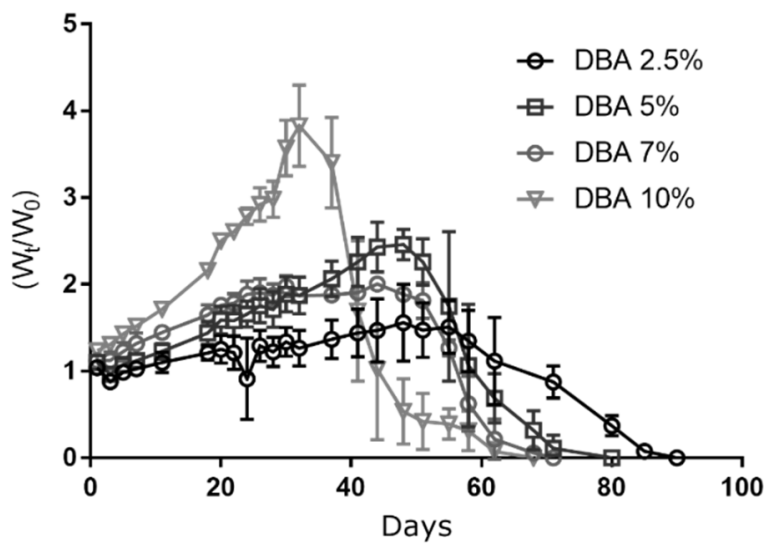

Figure 1: Swelling ratio $\left(W_{t} / W_{0}\right)$ in time of four different hydrogels (15 wt\%) formulations with 2.5, 5, 7 and 10\% DBA content in PNCD and PNND. Data are shown as mean \pm standard deviation $(n=3)$. 
degradable in physiological conditions and degradation time depended on the DBA ratio in the polymer structure (Fig. 1). These results clearly show the tunability of degradation time by varying the amount of DBA monomer. Preliminary data shows that these polymers can self-assemble into core crosslinked micelles with a Z-average diameter of $~ 85 \mathrm{~nm}$ (PDI 0.1). However, structural studies and degradation times of these micelles should be further investigated to get insight into the possibility for e.g. the development of polymeric micelles and/or hydrogels for local and targeted drug delivery.

\subsection{Drug conjugation to micelles}

In this thesis, we conjugated $\mathrm{N}$-hydroxysuccinimide (NHS) and maleimide functionalized dyes into the micellar core as a model drug. The conjugation was performed through the reaction of thiol or amine functionalities that remained after native chemical ligation in the micellar core with maleimide or NHS moieties in the dye. Recently, our group showed successful conjugation of a thiol functionalized drug to the core of star-like micelles crosslinked by native chemical ligation. Two complementary $A B$ block copolymers having $A$ block of PEG and B block of NIPAM and either HPMA-Cys or NAS were polymerized by RAFT. Then, the thiol moieties remaining after native chemical ligation were used for conjugation of gold nanoclusters and thiolated doxorubicin. A localized cytotoxic effect of this formulation on resilient MDA-MB-231 cancer cells triggered by near infra-red light irradiation was demonstrated ${ }^{52}$. Overall, the success of this method shows the potential of conjugating functionalized (bio)therapeutics into the micellar core and their use as responsive drug delivery systems.

\subsection{Reducing PEG immunogenicity by using PEG in loop conformation}

PEGylation is frequently used to improve the clinical properties of therapeutic protein $5^{53}$ and to enhance the circulation time of nanoparticles e.g. liposomes after intravenous administration ${ }^{54}$. However, in the last decade several unexpected immune-mediated sideeffects occurred with PEGylated therapeutics. For instance, the injection of PEGylated liposomes into mice, rats, beagle dogs, cynomolgus monkeys, and mini pigs can trigger a PEG-related immune response that is claimed to be elicited by anti-PEG antibodies ${ }^{55-58}$. The results presented in chapter 4 show that introducing PEG in a looped conformation in micelles significantly improves their circulation half-life in immunocompetent mice after both a single and second injection. Moreover, the lower binding of anti-PEG IgM to looped PEG compared to that of linear PEG (containing methoxy end groups) suggests the great potential of looped PEG in preventing or limiting the recognition by anti-PEG antibodies and 
thereby opsonization and clearance of PEGylated nanocarriers. It is hypothesized that due to the absence of PEG end group in this type of micelles and the lower avidity of the antiPEG IgM antibodies for looped PEG in flower-like micelles antibody binding and subsequent opsonization do not occur. It has been shown before that the PEG end group (particularly methoxy group) plays a role in triggering immune responses to PEGylated proteins and liposomes ${ }^{41,42}$. Therefore, it would be interesting to develop PEGylated liposomes with looped PEG on the corona. To this end, the synthesis of a PEG flanked by phospholipids from two sides is required. However, translation of this finding to the field of PEGylted proteins and peptides may be challenging due to possible crosslinking and aggregation of proteins and peptides through PEGylation with bifunctional PEG molecules. Further investigations however are required to obtain mechanistic insights into the generation of antibodies towards flower-like PEGylated micelles and the binding strength of these antibodies with PEGs of different conformations.

\subsection{Enzyme responsive hydrogels}

To develop the peptide polymer linker (Pep-NC) introduced in chapter 5, initially a peptide ATRP macroinitiator was synthesized via direct conjugation of an ATRP initiator to a peptide. Although the conjugation was successful, the resulting polymers showed a much higher molecular weight as determined by GPC than expected ( $\sim 5$ times higher than the $\mathrm{M}_{\mathrm{n}}$ based on the monomer to the initiator feed ratio) and high PDI, suggesting peptide aggregation during the polymerization. Moreover, the obtained polymers precipitated in water rather than forming a hydrogel due to the lack of hydrophilic segments. To address this issue, conjugation of the peptide to PEG was performed, which showed advantages over direct conjugation of ATRP initiator to the peptide. First, this method improved the solubility of the peptide in aqueous solutions and prevented aggregation of the peptide during polymerization. Second, the presence of PEG provided a hydrophilic segment in the polymer structure, which is essential to retain water in the formed 3D polymer network. The developed PEG-peptide macroinitiator was also successfully used for synthesis of a pentablock copolymer having PEG-peptide-PEG midblock and outer blocks of NIPAM and HPMA-ETSA (NIPAM-Co-HPMA-ETSA)-PEG-Peptide-PEG-P(NIPAM-co-HPMA-ETSA) (PepNE). Constructing a hydrogel using Pep-NC and Pep-NE can yield an enzyme responsive hydrogel which can be employed as scaffold in tissue engineering to mimic extracellular matrix. MMPs, including MMP-2 and MMP-9, are produced by endothelial cells and play an important role in regulating neovascularization and tissue remodeling ${ }^{59}$, 60 . Therefore, the described hydrogel has a high potential for support of vascularized tissue formation. 
Moreover, the introduced micellar hydrogel technology of chapter 5 can be easily used for development of other types of enzyme responsive micellar hydrogels. To this end, the peptide block in the linker can be substituted by a peptide that matches the specificity of the desired enzyme.

\subsection{Internalization by target cells}

As reported in chapter 3 and 6, cellular uptake of flower-like micelles is relatively low, likely caused by the PEGylated surface. This feature enables drug loaded micelles to circulate long and possibly reduces cytotoxicity because of their low uptake for healthy cells. To increase uptake of micelles by e.g. tumor cells, the micelles can be decorated with targeting ligands such as folic acid (FA) since folate receptors are upregulated on almost all cancer cells ${ }^{61}$. To prepare micelles with folic acid targeting ligand, micelles composed of $A B$ and $A B A$ block copolymers were developed and $A B$ copolymer carried folic acid. To this end, Boc-NH$\mathrm{PEG}_{3 \mathrm{kDa}}-\mathrm{OH}$ was used to produce (Boc-NH-PEG) ${ }_{2}-\mathrm{ABCPA}$ as a free radical polymerization macroinitiator. After Boc deprotection, an NHS functionalized folic acid was reacted with the free amine to yield a folic acid-PEG-ABCPA initiator. The obtained initiator was used for polymerization of NIPAM and HPMA-Cys yielding FA-PNC. The mixture of FA-PNC and PNE in aqueous solution at a temperature above the LCST of the polymers resulted in micelles with Z-average of $100 \mathrm{~nm}$ (PDI 0.15). However, further research is needed to evaluate the cellular uptake of these micelles by cells with upregulation of the folate receptor e.g. human nasopharyngeal epidermoid (KB) carcinoma cells. Moreover, the thiolated doxorubicin discussed in section 2.2 can be conjugated to these micelles and their in vivo efficacy can be evaluated in tumor-bearing animals.

\subsection{Large scale production}

The formulation of flower-like micelles using two complementary polymers in aqueous solution and at a moderate temperature $\left(50{ }^{\circ} \mathrm{C}\right)$ can possibly be performed using microchannel technology for large scale production of these micelles. Importantly, as shown in chapter 3 flower-like micelles can be easily lyophilized and micelles' size is hardly affected by this process. This feature provides the possibility of micelles' storage in the form of powder which is important for pharmaceutical production and storage . 


\section{Conclusion}

This thesis describes the development, characterization, and application of core crosslinked flower-like micelles and reports on their suitability for local and systemic pharmaceutical applications. It is demonstrated that PEG in a loop conformation reduces the immunogenicity of PEGylated micelles. It is further shown that core crosslinked flower-like micelles can be successfully formulated into enzyme-responsive hydrogels. Moreover, dense micellar dispersions of these micelles exhibit glassy behavior at a temperature below LCST with predictable $G^{\prime}$. The promising results described in this thesis encourage further research into application of flower-like micelles for drug delivery purposes. 


\section{References}

1.Sun, Q.; Barz, M.; De Geest, B. G.; Diken, M.; Hennink, W. E.; Kiessling, F.; Lammers, T.; Shi, Y., Nanomedicine and macroscale materials in immuno-oncology. Chemical Society Reviews 2019, 48 (1), 351-381.

2. Banik, B. L.; Fattahi, P.; Brown, J. L., Polymeric nanoparticles: the future of nanomedicine. Wiley Interdisciplinary Reviews: Nanomedicine and Nanobiotechnology 2016, 8 (2), 271299.

3. Pillai, O.; Panchagnula, R., Polymers in drug delivery. Current Opinion in Chemical Biology 2001, 5 (4), 447-451.

4.Sun, H.; Zhang, Y.; Zhong, Z., Reduction-sensitive polymeric nanomedicines: An emerging multifunctional platform for targeted cancer therapy. Advanced Drug Delivery Reviews 2018, 132, 16-32.

5.Talelli, M.; Barz, M.; Rijcken, C. J. F.; Kiessling, F.; Hennink, W. E.; Lammers, T., Corecrosslinked polymeric micelles: Principles, preparation, biomedical applications and clinical translation. Nano Today 2015, 10 (1), 93-117.

6.Talelli, M.; Rijcken, C. J. F.; Hennink, W. E.; Lammers, T., Polymeric micelles for cancer therapy: 3 C's to enhance efficacy. Current Opinion in Solid State and Materials Science 2012, 16 (6), 302-309.

7. Cabral, H.; Miyata, K.; Osada, K.; Kataoka, K., Block Copolymer Micelles in Nanomedicine Applications. Chemical Reviews 2018, 118 (14), 6844-6892.

8. Kataoka, K.; Harada, A.; Nagasaki, Y., Block copolymer micelles for drug delivery: Design, characterization and biological significance. Advanced Drug Delivery Reviews 2012, 64, 37 48.

9. Varela-Moreira, A.; Shi, Y.; Fens, M. H. A. M.; Lammers, T.; Hennink, W. E.; Schiffelers, R. M., Clinical application of polymeric micelles for the treatment of cancer. Materials Chemistry Frontiers 2017, 1 (8), 1485-1501.

10. Cabral, H.; Kataoka, K., Progress of drug-loaded polymeric micelles into clinical studies. Journal of Controlled Release 2014, 190, 465-476.

11. Hoare, T. R.; Kohane, D. S., Hydrogels in drug delivery: Progress and challenges. Polymer 2008, 49 (8), 1993-2007.

12. Hoffman, A. S., Hydrogels for biomedical applications. Advanced Drug Delivery Reviews 2012, 64, 18-23.

13. de Graaf, A. J.; Azevedo Prospero dos, S., II; Pieters, E. H.; Rijkers, D. T.; van Nostrum, C. F.; Vermonden, T.; Kok, R. J.; Hennink, W. E.; Mastrobattista, E., A micelleshedding thermosensitive hydrogel as sustained release formulation. Journal of Controlled Release 2012, 162 (3), 582-590. 
14. Ghoorchian, A.; Simon, J. R.; Bharti, B.; Han, W.; Zhao, X.; Chilkoti, A.; López, G. P., Bioinspired Reversibly Cross-linked Hydrogels Comprising Polypeptide Micelles Exhibit Enhanced Mechanical Properties. Advanced Functional Materials 2015, 25 (21), 3122-3130. 15. Abu Lila, A. S.; Kiwada, H.; Ishida, T., The accelerated blood clearance (ABC) phenomenon: Clinical challenge and approaches to manage. Journal of Controlled Release 2013, 172 (1), 38-47.

16. Heskins, M.; Guillet, J. E., Solution Properties of Poly(N-isopropylacrylamide). Journal of Macromolecular Science: Part A - Chemistry 1968, 2 (8), 1441-1455.

17. Akiyama, Y.; Kikuchi, A.; Yamato, M.; Okano, T., Ultrathin Poly(N-isopropylacrylamide) Grafted Layer on Polystyrene Surfaces for Cell Adhesion/Detachment Control. Langmuir 2004, 20 (13), 5506-5511.

18. Ebara, M.; Yamato, M.; Aoyagi, T.; Kikuchi, A.; Sakai, K.; Okano, T., TemperatureResponsive Cell Culture Surfaces Enable "On-Off" Affinity Control between Cell Integrins and RGDS Ligands. Biomacromolecules 2004, 5 (2), 505-510.

19. Uesugi, K.; Sakuma, Y.; Akiyama, Y.; Akiyama, Y.; Iwabuchi, K.; Okano, T.; Morishima, K., Temperature-responsive culture surfaces for insect cell sheets to fabricate a bioactuator. Advanced Robotics 2019, 33 (5), 219-231.

20. Qiu, S.; Zhuang, J.; Jin, S.; Yang, N.-L., Nitrocatecholic copolymers - synthesis and their remarkable binding affinity. Chemical Communications 2019, 55 (72), 10748-10751.

21. Song, F.; Wang, X.-L.; Wang, Y.-Z., Fabrication of novel thermo-responsive electrospun nanofibrous mats and their application in bioseparation. European Polymer Journal 2011, $47(10), 1885-1892$.

22. Islam, M. R.; Gao, Y.; Li, X.; Serpe, M. J., Responsive polymers for biosensing and protein delivery. Journal of Materials Chemistry B 2014, 2 (17), 2444-2451.

23. Xu, W.; Paidi, S. K.; Qin, Z.; Huang, Q.; Yu, C.-H.; Pagaduan, J. V.; Buehler, M. J.; Barman, I.; Gracias, D. H., Self-Folding Hybrid Graphene Skin for 3D Biosensing. Nano Letters 2019, 19 (3), 1409-1417.

24. Schmaljohann, D., Thermo- and pH-responsive polymers in drug delivery. Advanced Drug Delivery Reviews 2006, 58 (15), 1655-1670.

25. Shen, Y.; Kuang, M.; Shen, Z.; Nieberle, J.; Duan, H.; Frey, H., Gold Nanoparticles Coated with a Thermosensitive Hyperbranched Polyelectrolyte: Towards Smart Temperature and pH Nanosensors. Angewandte Chemie International Edition 2008, 47 (12), 2227-2230.

26. Wang, C.; Zhang, G.; Liu, G.; Hu, J.; Liu, S., Photo- and thermo-responsive multicompartment hydrogels for synergistic delivery of gemcitabine and doxorubicin. Journal of Controlled Release 2017, 259, 149-159.

27. de Graaf, A. J.; Mastrobattista, E.; Vermonden, T.; van Nostrum, C. F.; Rijkers, D. T. S.; Liskamp, R. M. J.; Hennink, W. E., Thermosensitive Peptide-Hybrid ABC Block Copolymers 
Obtained by ATRP: Synthesis, Self-Assembly, and Enzymatic Degradation. Macromolecules 2012, 45 (2), 842-851.

28. Qiu, Y.; Park, K., Environment-sensitive hydrogels for drug delivery. Advanced Drug Delivery Reviews 2012, 64, 49-60.

29. Ruskowitz, E. R.; Comerford, M. P.; Badeau, B. A.; DeForest, C. A., Logical stimulitriggered delivery of small molecules from hydrogel biomaterials. Biomaterials Science 2019, 7 (2), 542-546.

30. Matyjaszewski, K.; Xia, J., Atom Transfer Radical Polymerization. Chemical Reviews 2001, 101 (9), 2921-2990.

31. de Graaf, A. J.; Boere, K. W.; Kemmink, J.; Fokkink, R. G.; van Nostrum, C. F.; Rijkers, D. T.; van der Gucht, J.; Wienk, H.; Baldus, M.; Mastrobattista, E.; Vermonden, T.; Hennink, W. E., Looped structure of flowerlike micelles revealed by $1 \mathrm{H}$ NMR relaxometry and light scattering. Langmuir 2011, 27 (16), 9843-9848.

32. de Graaf, A. J., Self-assembled materials from thermosensitive and biohybrid block copolymers from controlled radical polymerization toward pharmaceutical applications. Thesis Utrecht University. 2012.

33. Hennink, W. E.; van Nostrum, C. F., Novel crosslinking methods to design hydrogels. Advanced Drug Delivery Reviews 2012, 64, 223-236.

34. Boere, K. W. M.; Soliman, B. G.; Rijkers, D. T. S.; Hennink, W. E.; Vermonden, T., Thermoresponsive Injectable Hydrogels Cross-Linked by Native Chemical Ligation. Macromolecules 2014, 47 (7), 2430-2438.

35. Dawson, P. E.; Muir, T. W.; Clark-Lewis, I.; Kent, S. B., Synthesis of proteins by native chemical ligation. Science 1994, 266 (5186), 776-779.

36. Najafi, M.; Kordalivand, N.; Moradi, M. A.; van den Dikkenberg, J.; Fokkink, R.; Friedrich, H.; Sommerdijk, N.; Hembury, M.; Vermonden, T., Native Chemical Ligation for Cross-Linking of Flower-Like Micelles. Biomacromolecules 2018, 19 (9), 3766-3775.

37. Lee, E. S.; Oh, K. T.; Kim, D.; Youn, Y. S.; Bae, Y. H., Tumor pH-responsive flower-like micelles of poly(l-lactic acid)-b-poly(ethylene glycol)-b-poly(I-histidine). Journal of Controlled Release 2007, 123 (1), 19-26.

38. Oh, K. T.; Oh, Y. T.; Oh, N.-M.; Kim, K.; Lee, D. H.; Lee, E. S., A smart flower-like polymeric micelle for $\mathrm{pH}$-triggered anticancer drug release. International Journal of Pharmaceutics 2009, 375 (1), 163-169.

39. Perry, J. L.; Reuter, K. G.; Kai, M. P.; Herlihy, K. P.; Jones, S. W.; Luft, J. C.; Napier, M.; Bear, J. E.; DeSimone, J. M., PEGylated PRINT Nanoparticles: The Impact of PEG Density on Protein Binding, Macrophage Association, Biodistribution, and Pharmacokinetics. Nano Letters 2012, 12 (10), 5304-5310. 
40. Soga, O.; $\quad$ van Nostrum, C. F.; Ramzi, A.; Visser, T.; Soulimani, F.; Frederik, P. M.; Bomans, P. H. H.; Hennink, W. E., Physicochemical Characterization of Degradable Thermosensitive Polymeric Micelles. Langmuir 2004, 20 (21), 9388-9395.

41. Shimizu, T.; Abu Lila, A. S.; Fujita, R.; Awata, M.; Kawanishi, M.; Hashimoto, Y.; Okuhira, K.; Ishima, Y.; Ishida, T., A hydroxyl PEG version of PEGylated liposomes and its impact on anti-PEG IgM induction and on the accelerated clearance of PEGylated liposomes. European Journal of Pharmaceutics and Biopharmaceutics 2018, 127, 142-149.

42. Sherman, M. R.; Williams, L. D.; Sobczyk, M. A.; Michaels, S. J.; Saifer, M. G. P., Role of the Methoxy Group in Immune Responses to mPEG-Protein Conjugates. Bioconjugate Chemistry 2012, 23 (3), 485-499.

43. Anonymous, Nature (London) 1972, 239, 488.

44. Torquato, S.; Truskett, T. M.; Debenedetti, P. G., Is Random Close Packing of Spheres Well Defined? Physical Review Letters 2000, 84 (10), 2064-2067.

45. Zaccone, A.; Terentjev, E. M., Disorder-Assisted Melting and the Glass Transition in Amorphous Solids. Physical Review Letters 2013, 110 (17), 178002-178007.

46. Minami, S.; Suzuki, D.; Urayama, K., Rheological aspects of colloidal gels in thermoresponsive microgel suspensions: formation, structure, and linear and nonlinear viscoelasticity. Current Opinion in Colloid \& Interface Science 2019, 43, 113-124.

47. Seymour, L. W.; Duncan, R.; Strohalm, J.; Kopeček, J., Effect of molecular weight (Mw) of $\mathrm{N}$-(2-hydroxypropyl)methacrylamide copolymers on body distribution and rate of excretion after subcutaneous, intraperitoneal, and intravenous administration to rats. Journal of Biomedical Materials Research 1987, 21 (11), 1341-1358.

48. Rijcken, C. J.; Snel, C. J.; Schiffelers, R. M.; van Nostrum, C. F.; Hennink, W. E., Hydrolysable core-crosslinked thermosensitive polymeric micelles: Synthesis, characterisation and in vivo studies. Biomaterials 2007, 28 (36), 5581-5593.

49. Cui, Z.; Lee, B. H.; Vernon, B. L., New Hydrolysis-Dependent Thermosensitive Polymer for an Injectable Degradable System. Biomacromolecules 2007, 8 (4), 1280-1286.

50. Neradovic, D.; van Steenbergen, M. J.; Vansteelant, L.; Meijer, Y. J.; van Nostrum, C. F.; Hennink, W. E., Degradation Mechanism and Kinetics of Thermosensitive Polyacrylamides Containing Lactic Acid Side Chains. Macromolecules 2003, 36 (20), 7491-7498.

51. Boere, K. W. M.; van den Dikkenberg, J.; Gao, Y.; Visser, J.; Hennink, W. E.; Vermonden, T., Thermogelling and Chemoselectively Cross-Linked Hydrogels with Controlled Mechanical Properties and Degradation Behavior. Biomacromolecules 2015, 16 (9), 2840-2851.

52. Hebels, E. R. N., M.; van den Dikkenberg, J.; Beztsinna, N.; Meeldijk, J.; Hembury, M.; Vermonden, T., Luminescent gold nanocluster-decorated polymeric hybrid particles for targeted combination therapy. In preparation 
53. Payne, R. W.; Murphy, B. M.; Manning, M. C., Product development issues for PEGylated proteins. Pharmaceutical Development and Technology 2011, 16 (5), 423-440.

54. Pasut, G.; Veronese, F. M., State of the art in PEGylation: The great versatility achieved after forty years of research. Journal of Controlled Release 2012, 161 (2), 461-472.

55. Dams, E. T. M.; Laverman, P.; Oyen, W. J. G.; Storm, G.; Scherphof, G. L.; van der Meer, J. W. M.; Corstens, F. H. M.; Boerman, O. C., Accelerated Blood Clearance and Altered Biodistribution of Repeated Injections of Sterically Stabilized Liposomes. Journal of Pharmacology and Experimental Therapeutics 2000, 292 (3), 1071-1079.

56. Ganson, N. J.; Povsic, T. J.; Sullenger, B. A.; Alexander, J. H.; Zelenkofske, S. L.; Sailstad, J. M.; Rusconi, C. P.; Hershfield, M. S., Pre-existing anti-polyethylene glycol antibody linked to first-exposure allergic reactions to pegnivacogin, a PEGylated RNA aptamer. Journal of Allergy and Clinical Immunology 2016, 137 (5), 1610-1613.

57. Suzuki, T.; Ichihara, M.; Hyodo, K.; Yamamoto, E.; Ishida, T.; Kiwada, H.; Ishihara, H.; Kikuchi, H., Accelerated blood clearance of PEGylated liposomes containing doxorubicin upon repeated administration to dogs. International Journal of Pharmaceutics 2012, 436 (1), 636-643.

58. Suzuki, T.; Ichihara, M.; Hyodo, K.; Yamamoto, E.; Ishida, T.; Kiwada, H.; Kikuchi, H.; Ishihara, H., Influence of dose and animal species on accelerated blood clearance of PEGylated liposomal doxorubicin. International Journal of Pharmaceutics 2014, 476 (1), 205-212.

59. Stratman, A. N.; Saunders, W. B.; Sacharidou, A.; Koh, W.; Fisher, K. E.; Zawieja, D. C.; Davis, M. J.; Davis, G. E., Endothelial cell lumen and vascular guidance tunnel formation requires MT1-MMP-dependent proteolysis in 3-dimensional collagen matrices. Blood 2009, $114(2), 237-247$.

60. Sokic, S.; Christenson, M. C.; Larson, J. C.; Appel, A. A.; Brey, E. M.; Papavasiliou, G., Evaluation of MMP substrate concentration and specificity for neovascularization of hydrogel scaffolds. Biomaterials Science 2014, 2 (10), 1343-1354.

61. Fernández, M.; Javaid, F.; Chudasama, V., Advances in targeting the folate receptor in the treatment/imaging of cancers. Chemical Science 2018, 9 (4), 790-810. 


\section{Appendices}

Nederlandse samenvatting

Curriculum vitae

List of publications

Acknowledgments 


\section{Nederlandse samenvatting}

Door innovaties binnen de polymeerchemie en de farmaceutische wetenschappen is het nu mogelijk om geavanceerde geneesmiddelafgiftesystemen te ontwerpen. Een voorbeeld hiervan zijn polymere micellen met een diameter tussen 10 en $100 \mathrm{~nm}$. Deze micellen worden gevormd door amfifiele blokcopolymeren in water te brengen, waarbij een hydrofobe kern wordt omringd door een hydrofiele buitenkant. Polymere micellen zijn uitermate geschikt voor geneesmiddelafgifte doordat hun karakteristieke fysischchemische eigenschappen aan te passen zijn aan de gewenste toepassing en verschillende polymere micellen worden dan ook onderzocht in klinische testen.

Een ander type polymere materialen met veelbelovende eigenschappen voor biomedische toepassingen zijn hydrogelen. Dit zijn hydrofiele netwerken van polymeren met een hoog watergehalte ( 70-99\% water). Een nieuwe trend in het onderzoek naar geneesmiddelafgiftesystemen is de combinatie van hydrogelen en micellen om onder andere een betere controle te krijgen over de afgiftesnelheid van geneesmiddelen.

Het doel van het werk beschreven in dit proefschrift was het ontwikkelen van een geavanceerd hydrogelsysteem dat in staat is om micellen af te geven door activatie van enzymen die aanwezig zijn in ziek weefsel.

Hoofdstuk 1 geeft een algemene introductie over polymere micellen en hoe hun stabiliteit beïnvloed kan worden door vernetting van de polymeerketens in de kern van de micel. Daarnaast wordt een overzicht gegeven hoe het polymeerblok polyethylene glycol (PEG) als hydrofiele buitenkant van de micel kan zorgen voor het zogenaamde 'stealth effect', waardoor micellen in de bloedcirculatie niet worden herkend en vervolgens verwijderd. Ondanks het stealth effect van PEG, treedt vaak een versnelde eliminatie van gePEGyleerde nanodeeltjes uit het bloed op bij een volgende toediening. Dit wordt het ABC ('accelerated blood clearance') effect genoemd. Daarnaast geeft dit hoofdstuk een introductie over hydrogelen die kunnen reageren op een externe prikkel en hun mogelijke toepassingen voor geneesmiddelafgifte.

Hoofdstuk 2 geeft een overzicht van de fysisch-chemische eigenschappen en biomedische toepassingen van materialen gebaseerd op poly( $\mathrm{N}$-isopropylacrylamide) (PNIPAM). Dit polymeer heeft thermosensitieve eigenschappen in een waterige omgeving. Het polymeer is goed oplosbaar in water beneden $32{ }^{\circ} \mathrm{C}$, maar assembleert bij temperaturen boven $32{ }^{\circ} \mathrm{C}$. Doordat deze overgangstemperatuur dichtbij lichaamstemperatuur zit, zijn er verschillende biomedische toepassingen voor materialen op basis van PNIPAM.

In hoofdstuk 3 wordt een chemische reactie gebruikt om de polymeerketens in de kern van micellen te vernetten. Deze reactie wordt 'native chemical ligation' (NCL) genoemd en bestaat uit de koppeling van een $\mathrm{N}$-terminale cysteine met een thioester, waarbij via een 
thioester tussenproduct uiteindelijk een amide wordt gevormd tussen de polymeerketens. De belangrijkste voordelen van deze reactie zijn dat er geen toxische katalysator nodig is en dat de reactie verloopt in water bij fysiologische $\mathrm{pH}$ en bij kamer- of lichaamstemperatuur. Twee complementaire ABA blokcopolymeren zijn gesynthetiseerd, waarbij het B-blok bestond uit PEG en de A-blokken bestonden uit NIPAM en N-(2hydroxypropyl)methacrylamide-ethylthioglycolate succinic acid (HPMA-ETSA) of NIPAM en $\mathrm{N}$-(2-hydroxypropyl)methacrylamide-cysteine (HPMA-Cys). Deze polymeren worden afgekort als PNC en PNE. Bloemvormige micellen (flower-like micelles genoemd in het Engels) werden gevormd door het mengen van deze polymeren in buffer en het verhogen van de temperatuur waarbij de A-blokken assembleren in de kern met lussen van de PEGB-blokken aan de buitenkant van de micel. Nadat native chemical ligation had opgetreden in de kern, werden micellen met een grootte van $\sim 65 \mathrm{~nm}$ gevormd bij lichaamstemperatuur. Deze micellen zijn verder onderzocht met cryo-transmissie elektronenmicroscopie en statische lichtverstrooiing waarbij uniforme ronde nanodeeltjes zijn waargenomen. Deze micellen zijn daarna voorzien van een fluorescent label, wat het mogelijk maakte ze te visualiseren. Celexperimenten lieten zien dat de deeltjes werden opgenomen door HeLa cellen, wat de mogelijke toepassing van deze micellen voor geneesmiddelafgifte laat zien. Bloem- en stervormige micellen zijn beide onderzocht als nano-geneesmiddelafgifte systemen, maar hun eigenschappen zijn nog nauwelijks met elkaar vergeleken. Daarom is in hoofdstuk 4 de circulatiekinetiek en biodistributie van zowel ster- als bloemvormige micellen in muizen onderzocht. Bloemvormige micellen zijn vervaardigd met de ABA blokcopolymeren zoals beschreven in hoofdstuk 3. Stervormige micellen daarentegen zijn vervaardigd van $A B$-blokcopolymeren met maar één $A$ blok met identieke eigenschappen als de $\mathrm{A}$ blokken van de bloemvormige micellen. In beide typen micellen is native chemical ligation toegepast om de ketens in de kern te vernetten. De grootte van de bloem- en stervormige micellen was respectievelijk 65 en $83 \mathrm{~nm}$. Daarnaast bleek uit statische lichtverstrooiing dat de bloemvormige micellen gemiddeld 400 polymeerketens bevatten en de stervormige 1700 polymeerketens. Het kleinere aantal polymeerketens in de bloemvormige micellen kan verklaard worden door de conformatie van de ketens in lussen die nu eenmaal meer ruimte innemen dan lineaire ketens. Opvallend was dat bloemvormige micellen langer in het bloed van BALB/c muizen gedetecteerd werden na intraveneuze injectie dan stervormige micellen ( $\mathrm{t} 1 / 2 \sim 18.6 \pm 3.1 \mathrm{vs.} 10.7 \pm 0.7 \mathrm{uur}$ ). De langere circulatietijd is waarschijnlijk gerelateerd aan de gereduceerde flexibiliteit van PEG in de lussen. Om te onderzoeken of dit ook het $A B C$ fenomeen beïnvloedt, zijn de muizen 7 dagen later nogmaals geïnjecteerd met ster- ofwel bloemvormige micellen. Na deze tweede injectie werd een versnelde eliminatie uit het bloed waargenomen voor de groep muizen die de tweede keer stervormige micellen hebben gekregen ongeacht welk type micellen in de 
eerste injectie was toegediend. Daarentegen, de muizen die bloemvormige micellen ontvingen in de tweede injectie lieten geen versnelde eliminatie uit het bloed zien. Om dit verschil in gedrag beter te bestuderen is een ELISA studie uitgevoerd met commerciële antiPEG antilichamen (IgM en IgG), waaruit bleek dat deze anti-PEG antilichamen wel binden aan stervormige micellen, maar niet aan bloemvormige. In bloedmonsters van muizen werd anti-PEG IgM gevonden 24 uur na de tweede injectie voor beide typen micellen. Gezien het verschil in eliminatiesnelheid uit de bloedstroom, wordt vermoed dat anti-PEG IgM op een andere manier bindt aan stervormige micellen dan aan bloemvormige micellen. Waarschijnlijk speelt de methoxy-eindgroep van PEG aan de stervormige micellen hierbij een rol. In de biodistributie studie zijn geen significante verschillen gevonden tussen de twee typen micellen. Beiden werden met name teruggevonden in de lever en de milt. Samenvattend toont deze studie aan dat de conformatie van PEG in een lus (waarbij er geen eindgroep aanwezig is) de circulatietijd van micellaire nanodeeltjes in het bloed kan verlengen en het $A B C$ fenomeen reduceert.

In hoofdstuk 5 is een enzymgevoelige hydrogel beschreven waarbij de bloemvormige micellen uit hoofdstuk 3 met elkaar zijn verbonden tot een driedimensionaal netwerk met behulp van een polymeerlinker. Deze linker bevat een L-peptideblok in het midden die geknipt kan worden door matrix metalloproteinases 2 en 9 . Het is bekend dat de concentratie van deze enzymen verhoogd is in ontstoken weefsels en tumoren. Als controle is er ook een hydrogel gemaakt met een D-peptide-linker (D-HyMic). Zoals verwacht, is er geen degradatie waargenomen voor de D-HyMic na blootstelling aan de genoemde enzymen. De hydrogelen met de L-peptide-linker (L-HyMic) degradeerden daarentegen binnen 7-45 dagen afhankelijk van de gebruikte concentratie collagenase, waarbij deeltjes vrijkwamen met een vergelijkbare grootte van de bloemvormige micellen. De vrijgekomen deeltjes zijn in staat om HeLa cellen binnen te dringen. Dit experiment illustreert de mogelijkheid om L-HyMic te gebruiken voor gecontroleerde afgifte van geneesmiddelen met een intracellulaire werking.

De bloemvormige micellen hebben een grootte die afhankelijk is van temperatuur. Boven de zogenaamde LCST ('lower critical solution temperature') hebben ze een radius van 35 $\mathrm{nm}$. Onder deze temperatuur neemt de radius toe tot $\sim 48 \mathrm{~nm}$ doordat de PNIPAM kern van de micellen wordt gehydrateerd. In hoofdstuk 6 zijn de reologische eigenschappen bestudeerd van geconcentreerde dispersies van deze bloemvormige micellen als functie van concentratie en de temperatuur. De miceldispersies vertoonden elastisch gedrag met een G' van 700, 3000 en 6000 Pa bij concentraties van 10, 12,5 en 15 gewichtsprocent. De effectieve volume fractie $\varphi=V m / V t$ is uitgerekend voor monsters met verschillende temperaturen en concentraties ( $V m$ is het totale volume van de micellen en $V t$ is het monstervolume). De waarde van $\varphi$ is gebruikt om de pakking van micellen te vergelijken 
bij verschillende concentraties en temperaturen. Doordat deze bolvormige micellen relatief zachte en indrukbare deeltjes zijn, is het mogelijk om meer deeltjes in een bepaald volume te persen dan theoretisch mogelijk zou zijn voor harde ronde deeltjes van dezelfde grootte. De effectieve volume fractie $(\varphi)$ bleek de enige parameter te zijn die de reologische eigenschappen van de dispersies bepaalt. In tegenstelling tot eerdere bevindingen met PNIPAM microgels, is hier geen precipitatie of gelering gevonden boven de LCST. Dit is te verklaren door de aanwezigheid van PEG aan de buitenkant van de micellen, dat interacties tussen PNIPAM ketens van verschillende micellen voorkomt.

\section{Conclusie}

Dit proefschrift beschrijft de ontwikkeling en karakterisering van bloemvormige micellen en beschrijft hun toepasbaarheid voor lokale en systemische farmaceutische toepassingen. De conformatie van PEG in een lus zorgt voor een gereduceerde immunogeniciteit van gePEGyleerde micellen. Bovendien is aangetoond dat deze micellen in een enzymgevoelige hydrogel konden worden ingebouwd en weer afgegeven worden bij blootstelling aan collagenase. Geconcentreerde dispersies van deze micellen gedroegen zich als een colloïdaal glas en hun reologische eigenschappen konden verklaard worden aan de hand van de grootte en de concentratie van de micellen. Samenvattend, de resultaten beschreven in dit proefschrift moedigen verder onderzoek naar bloemvormige micellen aan voor de ontwikkeling van nieuwe geneesmiddelafgiftesystemen. 



\section{Curriculum vitae}

Marzieh Najafi was born in 1983 in Qom, Iran. In 2001 she obtained her high school diploma and started her bachelor's program in chemistry at IKIU, Qazvin, Iran. She continued her education in organic chemistry at IASBS, Zanjan, Iran, under the supervision of Prof. dr. Babak Karimi and obtained her master's degree in this field in 2008. Between 2009 and 2013 she was working as a research assistant in the college of pharmacy in Zanjan. Marzieh and her family moved to the Netherlands in 2013 to start a new chapter in their life. In February 2014, she joined a research project at the department of Pharmaceutical Sciences at Utrecht University. Afterwards, Marzieh started her PhD research project in the same group under the supervision of Dr. ir. Tina Vermonden and Prof. dr. ir. Wim Hennink. The research focus was on investigating flower-like micelles for local and systemic pharmaceutical applications and the results are presented in this thesis. Now she continues her career in the field of nanomedicine in ARDENA-Oss, the Netherlands. 



\section{List of publications}

M. Shirangi, M. Najafi, D. T. S. Rijkers, R. J. Kok, W. E. Hennink, C. F. van Nostrum. Inhibition of octreotide acylation inside PLGA microspheres by derivatization of the amines of the peptide with a self-Immolative protecting group, Bioconjugate Chemistry 2016, 27, 3, 576585.

L. A. L. Fliervoet, M. Najafi, M. Hembury, T. Vermonden. Heterofunctional poly(ethylene glycol) (PEG) macroinitiator enabling controlled synthesis of $A B C$ triblock copolymers. Macromolecules, 2017, 50, 8390-8397.

M. Najafi, E. R. Hebels, W. E. Hennink, T. Vermonden. Poly ( $N$-isopropylacrylamide): physiochemical properties and biomedical applications, Book chapter, John Wiley \& sons publisher, July 2018.

M. Najafi, N. Kordalivand, M. A. Moradi, J. van den Dikkenberg, R. Fokkink, H. Friedrich, N.A.J.M. Sommerdijk, M. Hembury, T. Vermonden. Native chemical ligation for cross-linking of flower-like micelles. Biomacromolecules 2018, 19(9), 3766-3775.

E. R. Hebels, M. Najafi, J. van den Dikkenberg, N. Beztsinna, J. Meeldijk, M. Hembury, T. Vermonden. Luminescent gold nanocluster-decorated polymeric hybrid particles for targeted combination therapy. in preparation

M. Najafi, H. Asadi, J. van den Dikkenberg, M. J. van Steenbergen, M. H. A. M Fens, W. E. Hennink, T. Vermonden. Conversion of an injectable MMP-degradable hydrogel into corecrosslinked micelles. Biomacromolecules accepted manuscript, DOI: 10.1021/acs.biomac.9b01675 .

M. Najafi, M. Habibi, R. Fokkink, W. E. Hennink, T. Vermonden. Rheological properties of dense suspensions of thermosensitive core-crosslinked polymeric micelles. Submitted manuscript

M. Najafi, M. H. A. M. Fens, C. A. de Jongh, P. Boross, I. K. Ventouri, K. Dijkxhoorn, L. A. L. Fliervoet, A. A. Varela Moreira, R. Fokkink, A. Astefanei, P. J. Schoenmakers, C. F. van Nostrum, W. E. Hennink, T. Vermonden. The influence of PEG conformation in flower- and star-like micelles on circulation time and accelerated blood. in preparation 


\section{Selection of podium presentations}

M. Najafi, N. Kordalivand, J. van den Dikkenberg, R. Fokkink, M. Hembury, T. Vermonden. Core cross-linked flower-like micelles. European workshop on particulate systems in nanomedicine, Copenhagen, Denmark, January 2017.

M. Najafi, N. Kordalivand, M. A. Moradi, J. van den Dikkenberg, R. Fokkink, H. Friedrich, N.A.J.M. Sommerdijk, M. Hembury, T. Vermonden. Native chemical ligation for cross-linking of flower-like micelles. Dutch polymer days, Lunteren, the Netherland, April 2017, best presentation award.

M. Najafi, M. H. A. M. Fens, L. A. L. Fliervoet, A. A. Varela Moreira, R. Fokkink, W. E. Hennink, T. Vermonden. Physicochemical properties and biodistribution of flower- and star-like micelles, CHAINS, Veldhoven, the Netherland, December 2018.

M. Najafi, M. H. A. M. Fens, L. A. L. Fliervoet, A. A. Varela Moreira, R. Fokkink, W. E. Hennink, T. Vermonden. The influence of PEG conformation on properties of micelles. European Conference on Pharmaceutics, Bologna, Italy, March 2019. 


\section{Acknowledgements}

The work presented in this thesis is performed with enormous support and contribution of many people both scientifically and emotionally. Using this opportunity I would like to express my gratitude to all those people.

First and foremost, I would like to show my sincere gratitude to Tina. Dear Tina, thanks for being always positive and welcoming. Your scientific advice, patience, honesty, and trust had a tremendous impact on my personal and professional development. I always felt comfortable to share my thoughts with you and enjoyed working with you. You will always be my role model!

I would like to particularly thank Wim. Dear Wim, I am truly honored to be your student. I am impressed by your knowledge and dedication to science. Your critical comments and instructive inputs shaped my scientific mindset. Your enormous support especially during writing this thesis is unforgettable.

My special thanks to my best PhD buddies, Neda, Lucía, and Blessing. Your endless support and kind words mean a lot to me. I was very lucky to meet you during the PhD and would look forward to years of close friendship with you! No one could comfort me in the difficult days of work better than what you did. I love you girls! Negar, Dandan, Yinan, and Genoveva I am really grateful to have your support. Thanks for being always sweet and helpful. Dandan, your thesis template was very helpful to layout this book.

I would like to thank my great collaborators that I had the privilege to work with. Dear Marcel, thanks for all the work you did for animal study and for your endless support. Hamed, you did excellent research on peptide-polymer conjugation and you taught me RAFT polymerization, thanks for being so nice and helpful. Iro, your enthusiasm for the AF4 experiment was impressive. I very much enjoyed our friendly chat and wish to see your thesis soon! Mohammad-Amin, thanks for your great job on making Cryo-TEM images. Mathew, it was great to work with you on the same project and I learned golden science from you. Peter Boross, thanks for being so patient and teaching me about the immune system. Koichi Shiraishi, I enjoyed our discussion about anti-PEG antibodies. I wish you lots of success. Joep and Neda, thanks for all the work you did for cell studies and teaching me the basics of the work. Erik, Caroline, Rik, Joeri, Remco, Kim, and Allina, thanks for being always nice and helpful. 
I would like to thank our lovely technicians Mies, Joep, Roel, and Louis for their support in the daily lab work. Mies, you were always ready for my questions and to help me with research challenges. Thank you Barbara for your kind assistance in all the paperwork and administrative issues.

Lies, I should admit that without your help my PhD thesis could look different. I am glad to have the opportunity to collaborate with you. You greatly supported me during this period and I learned a lot from your organizational skills. You did a great job in the first animal study which made us motivated to continue in this direction. You also supported me in learning the Dutch language. Bedankt en ik wens je heel veel success!

It is a pleasure to thank my colleagues at the department of pharmaceutical sciences. Bo, you are a smart scientist and I learned a lot from you. I wish to see you in a place and position you like! Aida and Karina, you greatly supported me during the PhD and publishing the thesis. Karina, special thanks for all the job hunting tips! Ada, It was nice to have you in our group and wish you all the best with continuing the flower-like micelles project. Sjaak, Mehrnoosh, Mahsa, Sanaz, Kristel, Jeffrey, Jerry, Yvonne, Yanna, Haili, Nataliia, Xiangjie, Thijs, Vida, Yong, Yang, Menshang, Erik, Maria, Carl, Charis, Désireé, Gui, Andhyk, Feilong, thank you for always being nice and helpful, I really enjoyed our daily chat and spending time with you during various activities.

Living abroad is not easy without having good friends from your home country. Dear Sara, Narges, Fatemeh, Sareh, and Marzieh. We are getting old friends now! we shared a lot of ups and downs regarding being a working mom while living abroad. We are so close that my kids call you aunty. I am really proud of having you beside me. Thanks for all the support and love you gave me and my family. Dear Danial, thanks for designing the cover of my thesis and for your endless help and support. Also, I would like to thank Shayan, Majid, Shabnam, Mohamad-Amin, Naser, Vahid, Hodjat, Ali, Azadeh, Abbas, and Faeghe for the great moments we shared together and for your help during these years.

Annegina, thanks for all the flowers you brought for me when I was in my down days. You sent me photos of Aveed when I was busy at work and had no time for school activities, you made me feel I am there! I love our walking together with Michaela, and Herma and our endless chat about the Dutch culture, kids, education system, working mom, and etc. You made Wageningen my city! Michelle, you came to my life at the right time! Thanks for being so caring! 
Dear maman and baba, thanks for your endless and lifetime love and support. You thought me to work hard in my life and be nice and helpful to everyone. Maman Joon, this thesis would never come to an end if you could not come to help. Maryam, my dear sister, your strong and supportive personality, your kind and sympathetic nature always inspired me. Happiness is having you as a sister! Thanks for being always ready to help. Mostafa and Zahra I always enjoyed your company. Mostafa, according to Aveed you are the funniest uncle ever! Wish you lots of happiness! Dear maman Nazi and baba Gorban, you always warmly welcomed me in your place and were ready to help me at any moment. Thanks for your great support. Mehran and Farzaneh, I enjoyed all our chat and happy moment. Thanks for your kindness.

But last but not the least, I would like to thank Mehdi, Aveed, and Yara. Mehdi, my love, there are a lot of things in my life that would not have been accomplished without your support and this PhD is one of them. After moving to the Netherlands, we had years of hard work to build up our professional life while enjoying our personal life with our lovely daughters. I am really proud of having you beside me and looking forward to years of love and happiness. Lovely Aveed, when I started the PhD project, you were only two years old. I am sorry that I could not play with you whenever you wished or be present in the school activities, but you should know that mama is very proud of you and loves you a lot. Little Yara, you came to our life during this PhD. So, you are the only one from the family who was present when I was working in the lab! You also quickly learned to say: mama is working! Girls, I have good news for you; PHD LIFE IS OVER!

\section{Marzieh}


